\title{
Copper-Catalyzed Perfluoroalkylation of Alkynyl Bromides and Terminal Alkynes
}

Shilu Fan, ${ }^{*}$ Chenggong Zheng, Kaiting zheng, JunLan Li, Yaomei Liu, Fangpei Yan, Hua Xiao, Yi-Si Feng, ${ }^{*}$ Yuan-Yuan Zhu*

School of Chemistry and Chemical Engineering, Hefei University of Technology, 193 Tunxi Road, Anhui, 230000, People's Republic of China

Key Laboratory of Organofluorine Chemistry, Shanghai Institute of Organic Chemistry, Chinese Academy of Sci-ences, Shanghai, China

School of Food and Biological Engineering, Hefei University of Technology, 193 Tunxi Road, Anhui, 230000, People's Republic of China 


\section{List of Contents}

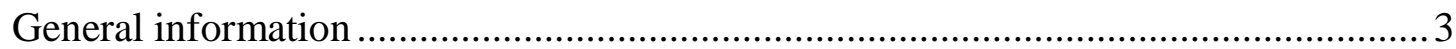

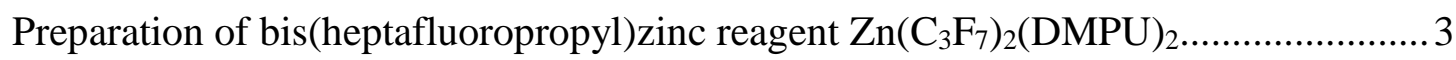

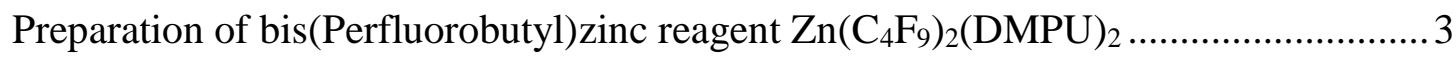

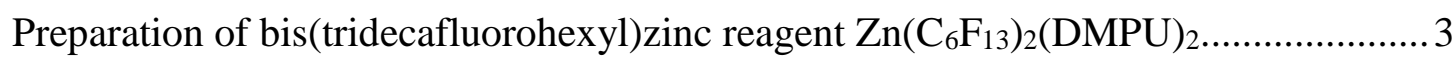

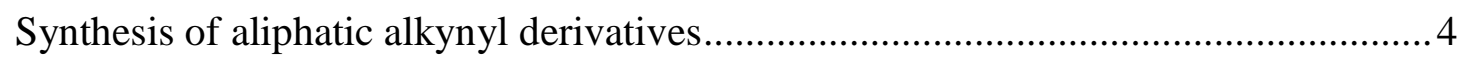

Synthesis of alkynyl derivatives of Steroids ........................................................ 5

Synthesis of (1S,2R,4R)-1-isopropyl-4-methyl-2-(prop-2-yn-1-yloxy)cyclohexane ....6

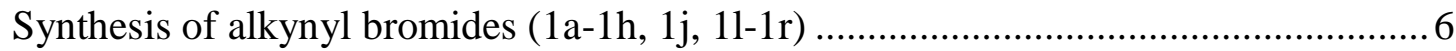

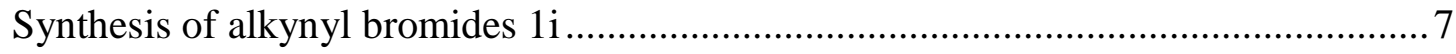

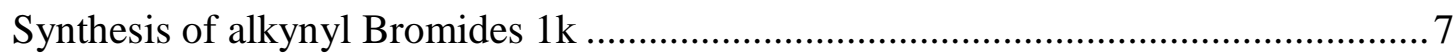

Representative results for optimization of copper-catalyzed perfluoroalkylation of

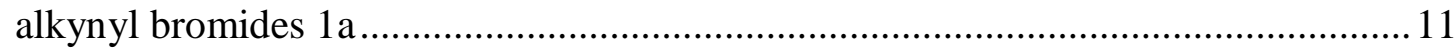

General procedure for copper-catalyzed perfluoroalkylation of alkynyl bromides ..... 12

One-pot synthesis of copper-catalyzed perfluoroalkylation reactions ........................22

Gram-scale synthesis of copper-catalyzed perfluoroalkylation of alkynyl bromides..23

The NMR spectroscopic monitoring of copper species using (DMPU) ${ }_{2} \mathrm{Zn}_{(}\left(\mathrm{C}_{4} \mathrm{~F}_{9}\right)_{2}$ and

$\mathrm{CuI}$

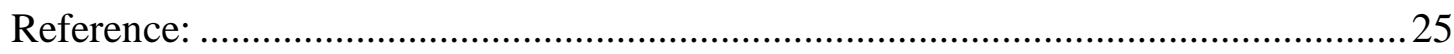

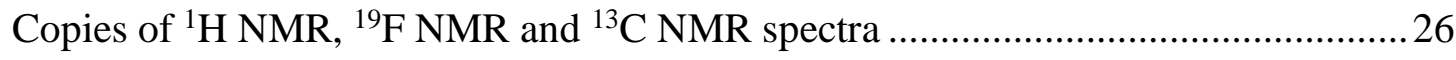


General information: ${ }^{1} \mathrm{H}$ NMR and ${ }^{13} \mathrm{C}$ NMR spectra were recorded on a Bruker 600 $\mathrm{MHz}$ spectrometer in $\mathrm{CDCl}_{3}$. Data for ${ }^{1} \mathrm{H}$ NMR are reported as follows: chemical shift (ppm, scale), multiplicity, coupling constant (Hz), and integration. Data for ${ }^{13} \mathrm{C} \mathrm{NMR}$ are reported in terms of chemical shift (ppm, scale), multiplicity, and coupling constant (Hz). High-resolution mass spectra were obtained by ESI on a TOF mass analyzer.

Materials: All reagents were used as received from commercial sources, unless specified otherwise, or prepared as described in the literature. All reagents were weighed and handled in air, and refilled with an inert atmosphere of $\mathrm{N}_{2}$ at room temperature. DMF, DMSO, DMPU were distilled under reduced pressure from $\mathrm{CaH}_{2}$ Toluene, 1,4-Dioxane, THF, Diglyme, and DME were distilled from sodium and benzophenone immediately before use.

\section{Preparation of bis(heptafluoropropyl)zinc reagent $\mathrm{Zn}\left(\mathrm{C}_{3} \mathrm{~F}_{7}\right)_{2}(\mathrm{DMPU})_{2}$}

To an oven-dried 100-mL two-neck round-bottomed flask equipped with a magnetic stir bar were added Toluene $(15 \mathrm{~mL})$ and DMPU $(2.4 \mathrm{~mL}, 20 \mathrm{mmol})$ under argon atmosphere. Heptafluoropropyl iodide $(3.6 \mathrm{~mL}, 25 \mathrm{mmol})$ was added to the solution. Diethyl zinc solution (1.0 M in hexanes, $10 \mathrm{~mL}, 10 \mathrm{mmol})$ was then added dropwise at $-41{ }^{\circ} \mathrm{C}$. After the reaction mixture was stirred at $-5{ }^{\circ} \mathrm{C}$ for $12 \mathrm{~h}$, the precipitate was obtained. After removing the solution, the precipitate obtained was washed with hexane $(50 \mathrm{~mL})$ three times and dried under vacuum to give $\mathrm{Zn}\left(\mathrm{C}_{3} \mathrm{~F}_{7}\right)_{2}(\mathrm{DMPU})_{2}$ as a white powder (6.0 g, 92\% yield).

\section{Preparation of bis(Perfluorobutyl)zinc reagent $\mathrm{Zn}\left(\mathrm{C}_{4} \mathrm{~F}_{9}\right)_{2}(\mathrm{DMPU})_{2}$}

To an oven-dried 100-mL two-neck round-bottomed flask equipped with a magnetic stir bar were added Toluene $(15 \mathrm{~mL})$ and DMPU $(2.4 \mathrm{~mL}, 20 \mathrm{mmol})$ under argon atmosphere. Perfluorobutyl iodide $(4.3 \mathrm{~mL}, 25 \mathrm{mmol})$ was added to the solution. Diethyl zinc solution (1.0 M in hexanes, $10 \mathrm{~mL}, 10 \mathrm{mmol}$ ) was then added dropwise at $-41{ }^{\circ} \mathrm{C}$. After the reaction mixture was stirred at $-5{ }^{\circ} \mathrm{C}$ for $12 \mathrm{~h}$, the precipitate was obtained. After removing the solution, the precipitate obtained was washed with hexane $(50 \mathrm{~mL})$ three times and dried under vacuum to give $\mathrm{Zn}\left(\mathrm{C}_{4} \mathrm{~F}_{9}\right)_{2}(\mathrm{DMPU})_{2}$ as a white powder (6.8 g, 88\% yield).

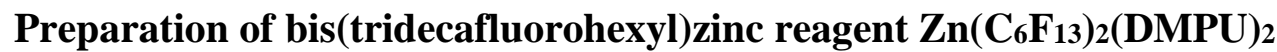


To an oven-dried 100-mL two-neck round-bottomed flask equipped with a magnetic stir bar were added Toluene (15 mL) and DMPU (2.4 mL, $20 \mathrm{mmol})$ under argon atmosphere. Tridecafluorohexyl iodide $(5.4 \mathrm{~mL}, 25 \mathrm{mmol})$ was added to the solution. Diethyl zinc solution (1.0 M in hexanes, $10 \mathrm{~mL}, 10 \mathrm{mmol}$ ) was then added dropwise at $-41{ }^{\circ} \mathrm{C}$. After the reaction mixture was stirred at $-5{ }^{\circ} \mathrm{C}$ for $12 \mathrm{~h}$, the precipitate was obtained. After removing the solution, the precipitate obtained was washed with hexane $(50 \mathrm{~mL})$ three times and dried under vacuum to give $\mathrm{Zn}\left(\mathrm{C}_{6} \mathrm{~F}_{13}\right)_{2}(\mathrm{DMPU})_{2}$ as a white powder $(8.3 \mathrm{~g}, 87 \%$ yield $)$.

\section{Synthesis of aliphatic alkynyl derivatives}

$$
\mathrm{HO}\left(\mathrm{H}_{2} \mathrm{C}\right)_{3} \equiv+\text { TBSCl} \frac{\text { imidazole }}{\mathrm{THF}, 0^{\circ} \mathrm{C} \text { to } \mathrm{rt}, 6 \mathrm{~h}} \quad \text { TBSO }\left(\mathrm{H}_{2} \mathrm{C}\right)_{3}=
$$

To a solution of pent-4-yn-1-ol (0.168 g, $2 \mathrm{mmol})$ in THF (15 mL) was added imidazole $(0.34 \mathrm{~g}, 5 \mathrm{mmol})$ followed by TBSCl $(0.32 \mathrm{~g}, 2.1 \mathrm{mmol})$ and the mixture stirred at room temperature overnight. Water $(300 \mathrm{~mL})$ was added and then the reaction mixture was extracted with $\mathrm{Et}_{2} \mathrm{O}(3 \times 300 \mathrm{~mL})$, dried $\left(\mathrm{Na}_{2} \mathrm{SO}_{4}\right)$, and evaporated under reduced pressure to leave a light yellow oil. The solvent was dissolved in petroleum ether and filtered through a short column of silica gel. Solvent was removed in vacuo to afford desired product.

$$
\mathrm{HO}\left(\mathrm{H}_{2} \mathrm{C}\right)_{3}=+\mathrm{Ac}_{2} \mathrm{O} \quad \frac{\mathrm{DMAP}}{\mathrm{DCM}, \mathrm{rt}, 6 \mathrm{~h}} \quad \mathrm{AcO}\left(\mathrm{H}_{2} \mathrm{C}\right)_{3}=
$$

To a solution of pent-4-yn-1-ol (0.168 g, 2 mmol) and DMAP (0.489 g, 4 mmol) in 10 $\mathrm{mL}$ of methylene chloride were added acetic anhydride $(0.856 \mathrm{~g}, 8.4 \mathrm{mmol})$. The solution was stirred at rt overnight and diluted with $10 \mathrm{~mL}$ of methylene chloride. The organic layer was washed with water, $2 \%$ of aqueous $\mathrm{HCl}$ solution, water again, and brine. Drying over $\mathrm{Na}_{2} \mathrm{SO}_{4}$ was followed by filtration and concentration, and purification by silica gel column chromatography provided product $0.167 \mathrm{~g}$. Yield $65 \%$.

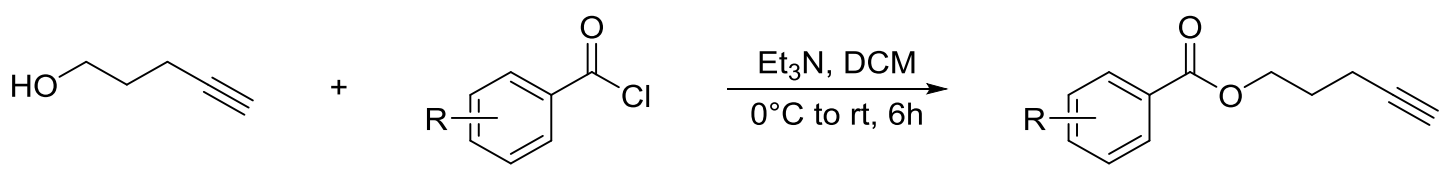

pent-4-yn-1-ol (0.168 g, 2 mmol) and $\mathrm{Et}_{3} \mathrm{~N}$ (0.42 g, $\left.4 \mathrm{mmol}\right)$ were added to DCM (10 $\mathrm{mL})$. Benzoyl chloride derivatives $(3 \mathrm{mmol})$ was added at $0{ }^{\circ} \mathrm{C}$, and the reaction mixture 
was allowed to stir at r.t. for $6 \mathrm{~h}$. The reaction was then quenched with water $(20 \mathrm{~mL})$, extracted with DCM $(3 \times 60 \mathrm{~mL})$. The combined organic layers were washed with brine $(60 \mathrm{~mL})$, dried $\left(\mathrm{Na}_{2} \mathrm{SO} 4\right)$, filtered and concentrated in vacuo. The crude residue was purified by flash column chromatography to afford desired product.

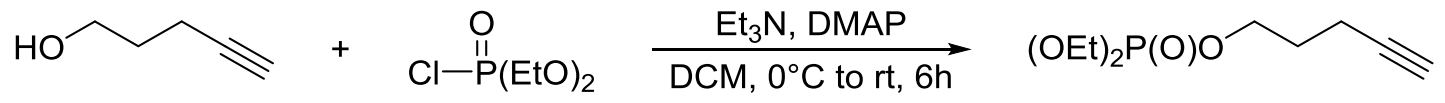

A flame-dried Schlenk flask is successively charged with the corresponding pent-4-yn1-ol (0.168 g, $2.0 \mathrm{mmol}), \mathrm{Et}_{3} \mathrm{~N}$ (0.303 g, $\left.3.0 \mathrm{mmol}\right)$, DMAP (0.061 g, $\left.0.5 \mathrm{mmol}\right)$, and $\mathrm{CH}_{2} \mathrm{Cl}_{2}(10 \mathrm{~mL})$. The reaction mixture is cooled to $0^{\circ} \mathrm{C}$ and diethyl chlorophosphate (1.5 equiv) is added dropwise to the solution. The resulting suspension is allowed to warm to room temperature and stirred for further $6 \mathrm{~h}$ at this temperature. After completion (TLC monitoring), the reaction is carefully quenched with saturated aqueous $\mathrm{NH}_{4} \mathrm{Cl}$ solution, and the aqueous phase is extracted with $\mathrm{CH}_{2} \mathrm{Cl}_{2}(3 \times 50 \mathrm{~mL})$. The combined organic phases are washed with water and dried over anhydrous $\mathrm{Na}_{2} \mathrm{SO}_{4}$.

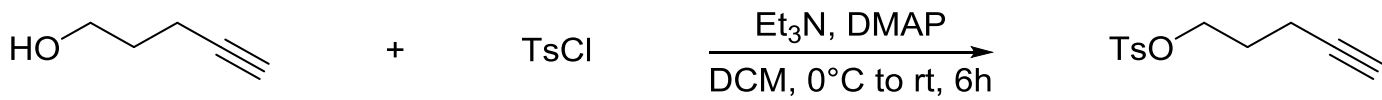

A flame-dried Schlenk flask is successively charged with the corresponding pent-4-yn1-ol $(0.168 \mathrm{~g}, 2.0 \mathrm{mmol}), \mathrm{Et}_{3} \mathrm{~N}(0.404 \mathrm{~g}, 4.0 \mathrm{mmol})$, and $\mathrm{CH}_{2} \mathrm{Cl}_{2}(10 \mathrm{~mL})$. The reaction mixture is cooled to $0{ }^{\circ} \mathrm{C}$ and 4-methylbenzenesulfonyl chloride (1.05 equiv) dissolved in $\mathrm{CH}_{2} \mathrm{Cl}_{2}(3 \mathrm{~mL})$ is added dropwise to the solution. The resulting suspension is allowed to warm to room temperature and stirred for further $6 \mathrm{~h}$ at this temperature. After completion (TLC monitoring), the reaction is carefully quenched with saturated aqueous $\mathrm{NH}_{4} \mathrm{Cl}$ solution, and the aqueous phase is extracted with $\mathrm{CH}_{2} \mathrm{Cl}_{2}(3 \times 50 \mathrm{~mL})$. The combined organic phases are washed with water and dried over anhydrous $\mathrm{Na}_{2} \mathrm{SO}_{4}$

\section{Synthesis of alkynyl derivatives of Steroids}<smiles>CC12CCC3c4ccc(O)cc4CCC3C1CCC2=O</smiles><smiles>[R]OC(=O)OCC(=O)OCc1ccccc1</smiles><smiles>C#CCOc1ccc2c(c1)CCC1C2CCC2(C)C(=O)CCC12</smiles>

A flame-dried Schlenk flask is successively charged with the corresponding estrone (0.54 g, $2.0 \mathrm{mmol}), \mathrm{K}_{2} \mathrm{CO}_{3}(0.33 \mathrm{~g}, 2.4 \mathrm{mmol})$, and acetone $(10 \mathrm{~mL})$, a magnetic stir bar 
under an atmosphere of nitrogen for $10 \mathrm{~min}$. Next, propargyl bromide (0.238 g, 2.0 mmol, 1.0 equiv) was slowly added at rt. The mixture was allowed to stir at $50{ }^{\circ} \mathrm{C}$ for $12 \mathrm{~h}$. After completion (TLC monitoring), the reaction was concentrated in vacuo. The soild residue was taken up in ethyl acetate $(100 \mathrm{ml})$ and washed twice with $\mathrm{H}_{2} \mathrm{O}(50 \mathrm{ml})$ and once with satd. aq $\mathrm{NaCl}(50 \mathrm{ml})$, before it was dried over $\mathrm{Na}_{2} \mathrm{SO}_{4}$. After evaporation of the solvent, silica gel column chromatography $(\mathrm{PE} /$ ethyle acetate $=10: 1)$ gave zcg02-01 (0.370 g, $60 \%)$ as a white solid.

\section{Synthesis of (1S,2R,4R)-1-isopropyl-4-methyl-2-(prop-2-yn-1-yloxy)cyclohexane}<smiles>CC(C)[C@H]1CC[C@@H](C)C[C@H]1O</smiles>

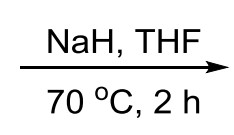
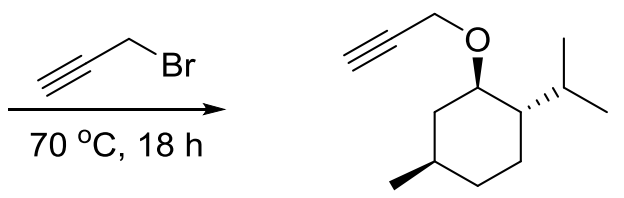

The procedure for the preparation of zcg-02-40 was operated by a literature procedure with a slight modification.1 A three-necked flask was charged with (L)-menthol (1.56 g, $10 \mathrm{mmol})$, tetrahydrofuran $(30 \mathrm{~mL}, 0.33 \mathrm{M})$, and a magnetic stir bar under an atmosphere of nitrogen. Next, 60\% $\mathrm{NaH}$ ( $0.8 \mathrm{~g}, 20 \mathrm{mmol}, 2.0$ equiv) was added in one portion at $0{ }^{\circ} \mathrm{C}$. The resulting heterogenous mixture was stirred vigorously at $70{ }^{\circ} \mathrm{C}$ for $2 \mathrm{~h}$, the reaction was allowed to cool to rt. Next, propargyl bromide $(2.2 \mathrm{~mL}, 25 \mathrm{mmol}$, 2.5 equiv) was slowly added at rt. The mixture was allowed to stir at $70{ }^{\circ} \mathrm{C}$ for $18 \mathrm{~h}$. After completion $[\mathrm{Rf}=0.3$ (Petroleum ether $(100 \%)$, stained with $\mathrm{KMnO} 4$ in TLC monitoring], the reaction is carefully quenched with saturated aqueous $\mathrm{NH} 4 \mathrm{Cl}$ solution $(30 \mathrm{~mL})$, and the aqueous phase is extracted with ethyl acetate $(3 \times 50 \mathrm{~mL})$. The combined organic phases are washed with water and dried over anhydrous $\mathrm{Na} 2 \mathrm{SO} 4$ and filtered and concentrated. The residue was purified with silica gel chromatography (Petroleum ether (100\%)) to provide pure product (528 mg, 27\%) as a slightly yellow oil.

\section{Synthesis of alkynyl bromides (1a-1h, 1j, 1l-1r)}

$$
\mathrm{R} \rightleftharpoons \quad \text { NBS } \quad \frac{\mathrm{AgNO}_{3}(10 \mathrm{~mol} \%)}{\text { acetone, } \mathrm{rt}, 3 \mathrm{~h}} \quad \mathrm{R} \rightleftharpoons \mathrm{Br}
$$

$\mathrm{AgNO}_{3}(0.017 \mathrm{~g}, 0.1 \mathrm{mmol})$ was added to a solution of alkynyl derivatives (1mmol) in acetone $(5 \mathrm{~mL})$. Then NBS $(0.196 \mathrm{~g}, 1.1 \mathrm{mmol})$ was added in portion. The mixture was stirred for $3 \mathrm{~h}$ at room temperature, and then concentrated in vacuo. The solvent was 
dissolved in petroleum ether and filtered through a short column of silica gel. Solvent was removed in vacuo to afford desired product.

\section{Synthesis of alkynyl bromides 1i}

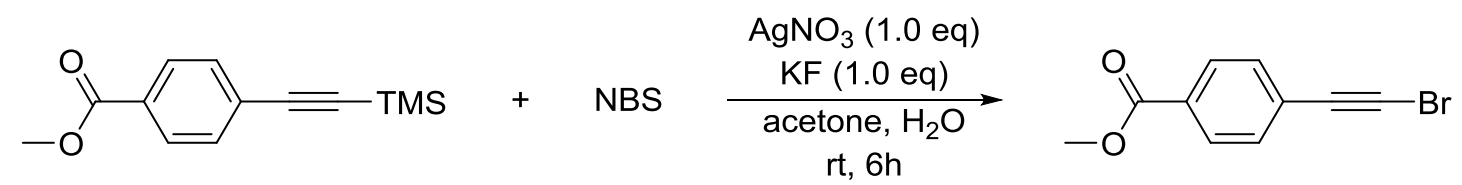

To the solution of methyl 4-((trimethylsilyl)ethynyl)benzoate $(0.232 \mathrm{~g}, 1.0 \mathrm{mmol})$ in 6 $\mathrm{mL}$ of acetonitrile, water $(36 \mu \mathrm{L}, 2 \mathrm{mmol})$ was added under $\mathrm{N}_{2}$. Next $\mathrm{AgNO}_{3}(0.178 \mathrm{~g}$, $1.0 \mathrm{mmol})$ and $\mathrm{KF}(0.058 \mathrm{~g}, 1.0 \mathrm{mmol})$ and NBS (2.43 g, $10.4 \mathrm{mmol})$ were added. The reaction was stirred for $6 \mathrm{~h}$ at room temperature without an access of light. The solvent was removed under reduced pressure and the residue was purified by elution through silica gel $\left(\mathrm{CH}_{2} \mathrm{Cl}_{2}\right)$ yielding $227 \mathrm{mg}(0.95 \mathrm{mmol}, 95 \%)$ of white solid.

\section{Synthesis of alkynyl Bromides 1k}

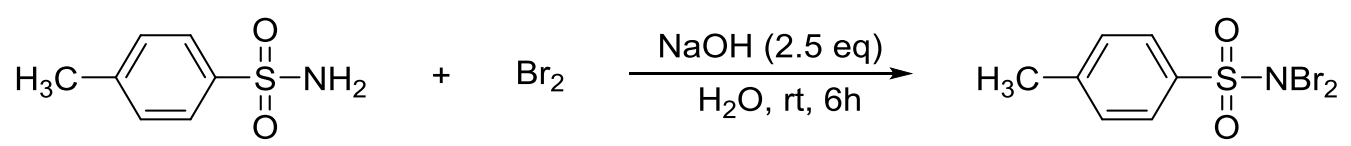

4-Methylbenzenesulphonimide (2.12 g, $12 \mathrm{mmol})$ was dissolved in a solution of $\mathrm{NaOH}$ $(1.2 \mathrm{~g}, 30 \mathrm{mmol})$ in water $(25 \mathrm{~mL})$ at room temperature. Then bromine $(2.17 \mathrm{~mL})$ was added with vigorous stirring. The resulting precipitate of N,N-dibromo-4methylbenzenesulphonimide was filtrated and washed with cold water. Recrystallization with ethanol afforded pure reagent in $80 \%$ yield.

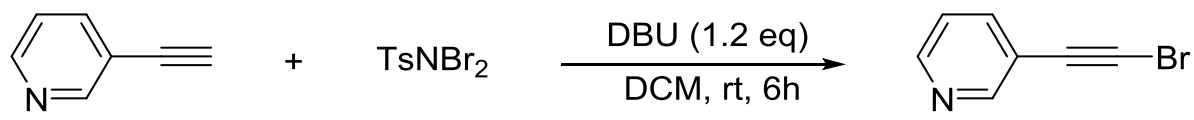

To a stirred solution of 3-ethynylpyridine (1 mmol) in $\mathrm{CH}_{2} \mathrm{Cl}_{2}(3 \mathrm{ml}), \mathrm{TsNBr}_{2}(1.2$ $\mathrm{mmol})$ and DBU (1.2 mmol) was added. The progress of the reaction was monitored by TLC. After the completion of the reaction sodium thiosulfate $(200 \mathrm{mg})$ was added to the reaction mixture and further stirred for 5 mins. The reaction mixture was washed with water and extracted with ethylacetate. The organic layer was separated and dried over $\mathrm{Na}_{2} \mathrm{SO}_{4}$ and concentrated in vaccuo. The solvent was removed under reduced pressure and the residue was purified by elution through silica gel (Petroleum ether / Ethyl acetate $=3: 1$ ) yielding $107 \mathrm{mg}$ (yield 59\%) of brown solid. 


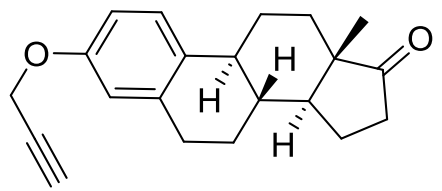

3-O-Propagylestrone. This compound is known. ${ }^{1} 1 \mathrm{H}$ NMR $(600 \mathrm{MHz}, \mathrm{CDCl} 3) \delta 7.22$ $(\mathrm{d}, J=8.6 \mathrm{~Hz}, 1 \mathrm{H}), 6.79(\mathrm{dd}, J=8.6 \mathrm{~Hz}, 2.8 \mathrm{~Hz}, 1 \mathrm{H}), 6.72(\mathrm{~d}, 2.8 \mathrm{~Hz}, 1 \mathrm{H}), 4.66(\mathrm{~d}, J$ $=2.4 \mathrm{~Hz}, 2 \mathrm{H}), 2.90(\mathrm{~m}, 2 \mathrm{H}), 2.51(\mathrm{~m}, 2 \mathrm{H}), 2.39(\mathrm{~m}, 1 \mathrm{H}), 2.25(\mathrm{~m}, 1 \mathrm{H}), 2.14(\mathrm{~m}, 1 \mathrm{H})$, $2.01(\mathrm{~m}, 3 \mathrm{H}), 1.63(\mathrm{~m}, 2 \mathrm{H}), 1.52-1.39(\mathrm{~m}, 4 \mathrm{H}), 0.93(\mathrm{~s}, 3 \mathrm{H})$.<smiles>C#CCOC1CC(C)CC[C@H]1C(C)C</smiles>

(1S,2R,4R)-1-isopropyl-4-methyl-2-(prop-2-yn-1-yloxy)cyclohexane.

This compound is known. ${ }^{2}{ }^{1} \mathrm{H} \mathrm{NMR}(600 \mathrm{MHz}$, Chloroform- $d) \delta 4.24-4.16(\mathrm{~m}, 1 \mathrm{H}), 4.14$ (dd, $J$ $=15.9,2.3 \mathrm{~Hz}, 1 \mathrm{H}), 3.25(\mathrm{td}, J=10.6,4.2 \mathrm{~Hz}, 1 \mathrm{H}), 2.36(\mathrm{td}, J=2.4,0.8 \mathrm{~Hz}, 1 \mathrm{H}), 2.21(\mathrm{~m}, 1 \mathrm{H})$, $2.09(\mathrm{~m}, 1 \mathrm{H}), 1.63(\mathrm{~m}, 2 \mathrm{H}), 1.35(\mathrm{~m}, 1 \mathrm{H}), 1.21(\mathrm{~m}, 1 \mathrm{H}), 0.99(\mathrm{~m}, 1 \mathrm{H}), 0.91(\mathrm{~d}, J=6.5 \mathrm{~Hz}, 3 \mathrm{H})$, $0.4(\mathrm{~m}, 2 \mathrm{H}) 0.89(\mathrm{~d}, \mathrm{~J}=7.0 \mathrm{~Hz}, 3 \mathrm{H}) .0 .79(\mathrm{~d}, \mathrm{~J}=6.9 \mathrm{~Hz}, 3 \mathrm{H})$.

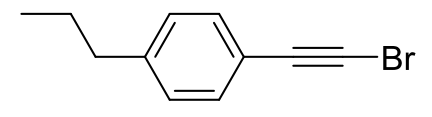

(E)- 1-(Bromoethynyl)-4-propylbenzene (1a). This compound is known. ${ }^{3} \mathrm{H}$ NMR $\left(600 \mathrm{MHz}, \mathrm{CDCl}_{3}\right) \delta 7.37(\mathrm{~d}, J=8.4 \mathrm{~Hz}, 2 \mathrm{H}), 7.11(\mathrm{~d}, J=7.8 \mathrm{~Hz}, 2 \mathrm{H}), 2.57$ (t, $J=7.8$ $\mathrm{Hz}, 2 \mathrm{H}), 5.61(\mathrm{dt}, J=14.4 \mathrm{~Hz}, 7.8 \mathrm{~Hz}, 2 \mathrm{H}), 0.93$ (t, J = 7.8 Hz, 3H).<smiles>CCCCCCCCCCCC#Cc1ccc(C#CBr)cc1</smiles>

1-(Bromoethynyl)-4-pentylbenzene (1b). This compound is known. ${ }^{4}{ }^{1} \mathrm{H}$ NMR (600 $\left.\mathrm{MHz} \mathrm{CDCl}_{3}\right) \delta 7.36(\mathrm{~d}, J=8.4 \mathrm{~Hz}, 2 \mathrm{H}), 7.12(\mathrm{~d}, J=8.4 \mathrm{~Hz}, 2 \mathrm{H}), 2.58(\mathrm{t}, J=7.8 \mathrm{~Hz}$, 2H), 1.59 (dt, $J=15.0 \mathrm{~Hz}, 7.8 \mathrm{~Hz}, 2 \mathrm{H}), 1.30$ (m, 4H), 0.89 (t, $J=7.2 \mathrm{~Hz}, 3 \mathrm{H})$.<smiles>BrC#Cc1ccccc1</smiles>

(Bromoethynyl)benzene (1c). This compound is known. ${ }^{5}{ }^{1} \mathrm{H} \mathrm{NMR}\left(600 \mathrm{MHz}, \mathrm{CDCl}_{3}\right)$ $\delta 7.48(\mathrm{~d}, J=7.8 \mathrm{~Hz}, 2 \mathrm{H}), 7.35(\mathrm{~m}, 3 \mathrm{H})$.<smiles>COc1ccc(C#CBr)cc1</smiles>

Methyl 4-(bromoethynyl)benzoate (1d). This compound is known. ${ }^{3}{ }^{1} \mathrm{H}$ NMR (600 $\left.\mathrm{MHz}, \mathrm{CDCl}_{3}\right) \delta 7.38(\mathrm{~d}, \mathrm{~J}=8.8 \mathrm{~Hz}, 2 \mathrm{H}), 6.83(\mathrm{~d}, \mathrm{~J}=8.8 \mathrm{~Hz}, 2 \mathrm{H}), 3.81(\mathrm{~s}, 3 \mathrm{H})$. 


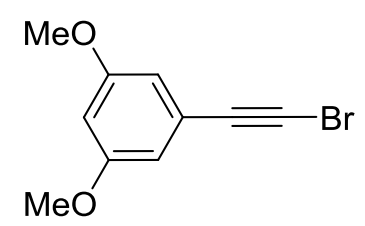

1-(Bromoethynyl)-4-methoxybenzene (1e). This compound is known. ${ }^{3}{ }^{1} \mathrm{H}$ NMR (600 $\left.\mathrm{MHz}, \mathrm{CDCl}_{3}\right) \delta 6.60(\mathrm{~d}, J=2.3 \mathrm{~Hz}, 2 \mathrm{H}), 6.46(\mathrm{~d}, J=2.3 \mathrm{~Hz}, 1 \mathrm{H}), 3.77(\mathrm{~s}, 6 \mathrm{H})$.

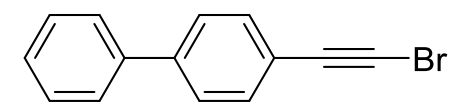

4-(Bromoethynyl)-1,1'-biphenyl (1f). This compound is known. ${ }^{4} \mathrm{H}$ NMR (600 MHz, $\left.\mathrm{CDCl}_{3}\right) \delta 7.59(\mathrm{~d}, J=8.4 \mathrm{~Hz}, 2 \mathrm{H}), 7.55(\mathrm{~d}, J=8.4 \mathrm{~Hz}, 2 \mathrm{H}), 7.52(\mathrm{~d}, J=7.8 \mathrm{~Hz}, 2 \mathrm{H})$, $7.45(\mathrm{t}, J=7.2 \mathrm{~Hz}, 2 \mathrm{H}), 7.37$ (t, $J=7.2 \mathrm{~Hz}, 1 \mathrm{H})$.

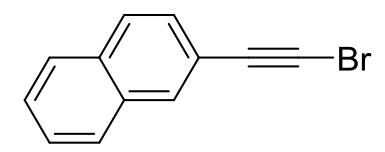

2-(Bromoethynyl)naphthalene (1g). This compound is known. ${ }^{6} \mathrm{H}$ NMR $(600 \mathrm{MHz}$, $\left.\mathrm{CDCl}_{3}\right) \delta 7.99(\mathrm{~s}, 3 \mathrm{H}), 7.79(\mathrm{~m}, 1 \mathrm{H}), 7.49(\mathrm{~m}, 3 \mathrm{H})$.<smiles>Clc1ccc(C#CBr)cc1</smiles>

1-(Bromoethynyl)-4-chlorobenzene (1h). This compound is known. ${ }^{7} \mathrm{H}$ NMR (600 $\left.\mathrm{MHz}, \mathrm{CDCl}_{3}\right) \delta 7.37(\mathrm{~d}, J=9.0 \mathrm{~Hz}, 2 \mathrm{H}), 7.29(\mathrm{~d}, J=8.4 \mathrm{~Hz}, 2 \mathrm{H})$.

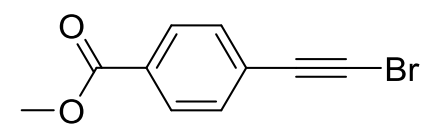

methyl 4-(bromoethynyl)benzoate (1i). This compound is known. ${ }^{7} \mathrm{H}$ NMR (600 $\left.\mathrm{MHz}, \mathrm{CDCl}_{3}\right) \delta 6.60(\mathrm{~d}, J=2.4 \mathrm{~Hz}, 1 \mathrm{H}), 6.46(\mathrm{t}, J=2.4 \mathrm{~Hz}, 1 \mathrm{H}), 3.77(\mathrm{~s}, 6 \mathrm{H})$.<smiles>BrC#Cc1ccsc1</smiles>

3-(Bromoethynyl)thiophene (1j). This compound is known. ${ }^{7} \mathrm{H}$ NMR (600 MHz, $\left.\mathrm{CDCl}_{3}\right) \delta 7.48(\mathrm{~d}, J=3.0 \mathrm{~Hz}, 1 \mathrm{H}), 7.25(\mathrm{~m}, 1 \mathrm{H}), 7.12(\mathrm{~d}, J=4.8 \mathrm{~Hz}, 1 \mathrm{H})$.<smiles>BrC#Cc1cccnc1</smiles>

3-(Bromoethynyl)pyridine (1k). This compound is known. ${ }^{3}{ }^{1} \mathrm{H}$ NMR (600 MHz, $\left.\mathrm{CDCl}_{3}\right) \delta 8.67(\mathrm{~s}, 1 \mathrm{H}), 8.53(\mathrm{~d}, J=4.8 \mathrm{~Hz}, 1 \mathrm{H}), 7.71(\mathrm{~d}, J=7.8 \mathrm{~Hz}, 1 \mathrm{H}), 7.22(\mathrm{~m}, 1 \mathrm{H})$. $\mathrm{H}_{3} \mathrm{C}\left(\mathrm{CH}_{2}\right)_{9}=\mathrm{Br}$

1-Bromododec-1-yne (11). This compound is known. ${ }^{8}{ }^{1} \mathrm{H} \mathrm{NMR}\left(600 \mathrm{MHz}, \mathrm{CDCl}_{3}\right) \delta$ $2.18(\mathrm{t}, J=7.2 \mathrm{~Hz}, 2 \mathrm{H}), 1.49(\mathrm{dt}, J=15.0 \mathrm{~Hz}, 7.2 \mathrm{~Hz}, 2 \mathrm{H}), 1.35(\mathrm{~m}, 2 \mathrm{H}), 1.27(\mathrm{~m}, 13 \mathrm{H})$, 
$0.87(\mathrm{t}, J=6.6 \mathrm{~Hz}, 3 \mathrm{H})$

$\operatorname{TBSO}\left(\mathrm{CH}_{2}\right)_{3}=\mathrm{Br}$

((5-Bromopent-4-yn-1-yl)oxy)(tert-butyl)dimethylsilane (1m). This compound is known. ${ }^{9}{ }^{1} \mathrm{H}$ NMR $\left(600 \mathrm{MHz}, \mathrm{CDCl}_{3}\right) \delta 3.66(\mathrm{t}, J=6.0 \mathrm{~Hz}, 2 \mathrm{H}), 2.28(\mathrm{t}, J=7.2 \mathrm{~Hz}, 2 \mathrm{H})$, $1.69(\mathrm{dt}, J=13.2 \mathrm{~Hz}, 6.0 \mathrm{~Hz}, 2 \mathrm{H}), 0.88(\mathrm{~s}, 9 \mathrm{H}), 0.04(\mathrm{~s}, 6 \mathrm{H})$.

$\mathrm{AcO}\left(\mathrm{CH}_{2}\right)_{3}=\mathrm{Br}$

5-Bromopent-4-yn-1-yl acetate (1n). This compound is known. ${ }^{3}{ }^{1} \mathrm{H}$ NMR $(600 \mathrm{MHz}$, $\left.\mathrm{CDCl}_{3}\right) \delta 4.12(\mathrm{~m}, 2 \mathrm{H}), 2.29(\mathrm{~m}, 2 \mathrm{H}), 2.03(\mathrm{~s}, 3 \mathrm{H}), 1.83(\mathrm{~m}, 2 \mathrm{H})$.

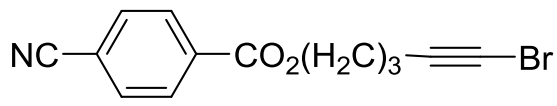

5-Bromopent-4-yn-1-yl 4-cyanobenzoate (1o). ${ }^{1} \mathrm{H}$ NMR $\left(600 \mathrm{MHz}, \mathrm{CDCl}_{3}\right) \delta 8.14(\mathrm{~d}$, $J=8.4 \mathrm{~Hz}, 2 \mathrm{H}), 7.74(\mathrm{~d}, J=7.8 \mathrm{~Hz}, 2 \mathrm{H}), 4.44$ (t, $J=6.0 \mathrm{~Hz}, 2 \mathrm{H}), 2.40(\mathrm{t}, J=7.2 \mathrm{~Hz}$, 2H), $2.00(\mathrm{dt}, J=13.2 \mathrm{~Hz}, J=6.6 \mathrm{~Hz}, 2 \mathrm{H}) .{ }^{13} \mathrm{C} \mathrm{NMR}\left(150.8 \mathrm{MHz}, \mathrm{CDCl}_{3}\right) \delta 164.8$, $133.9,132.2,130.0,117.9,116.4,78.6,64.4,39.1,27.2,16.7$. IR (thin film): $v_{\max } 2231$, 1716, $761 \mathrm{~cm}^{-1}$. MS (EI) m/z: (\%) 290 (M), 130, 65 (100). HRMS (EI) m/z: [M] $]^{+}$ Calculated for $\mathrm{C}_{13} \mathrm{H}_{10} \mathrm{BrNO}_{2}$ : 290.9859; Found: 290.9864 .

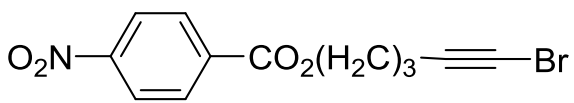

5-Bromopent-4-yn-1-yl 4-nitrobenzoate (1p). ${ }^{1} \mathrm{H}$ NMR $\left(600 \mathrm{MHz}, \mathrm{CDCl}_{3}\right) \delta 8.27(\mathrm{~d}$, $J=8.4 \mathrm{~Hz}, 2 \mathrm{H}), 8.19(\mathrm{~d}, J=8.4 \mathrm{~Hz}, 2 \mathrm{H}), 4.45(\mathrm{t}, J=6.0 \mathrm{~Hz}, 2 \mathrm{H}), 2.40(\mathrm{t}, J=6.6 \mathrm{~Hz}$, 2H), $2.00(\mathrm{dt}, J=13.2 \mathrm{~Hz}, J=6.6 \mathrm{~Hz}, 2 \mathrm{H}) .{ }^{13} \mathrm{C} \mathrm{NMR}\left(150.8 \mathrm{MHz}, \mathrm{CDCl}_{3}\right) \delta 164.6$, $150.5,135.5,130.7,123.5,78.6,64.5,39.1,27.3,16.7$. IR (thin film): $v_{\max } 1714,1248$, $717 \mathrm{~cm}^{-1}$. MS (EI) m/z: (\%) 310 (M), 150, 65 (100). HRMS (APCI-LTQ Orbitrap) m/z: $[\mathrm{M}+\mathrm{H}]^{+}$Calculated for $\mathrm{C}_{12} \mathrm{H}_{11} \mathrm{BrNO}_{4}$ : 311.9866; Found: 311.9867 . $(\mathrm{EtO})_{2} \mathrm{OPO}\left(\mathrm{CH}_{2}\right)_{3}=\mathrm{Br}$

5-Bromopent-4-yn-1-yl diethyl phosphate (1q). ${ }^{1} \mathrm{H}$ NMR $\left(600 \mathrm{MHz}, \mathrm{CDCl}_{3}\right) \delta 4.08$ $(\mathrm{m}, 6 \mathrm{H}), 2.31(\mathrm{t}, J=7.2 \mathrm{~Hz}, 2 \mathrm{H}), 1.83(\mathrm{dt}, J=13.2 \mathrm{~Hz}, J=6.0 \mathrm{~Hz}, 2 \mathrm{H}), 1.30(\mathrm{t}, J=$ $7.2 \mathrm{~Hz}, 6 \mathrm{H}) .{ }^{13} \mathrm{C}$ NMR $\left(150.8 \mathrm{MHz}, \mathrm{CDCl}_{3}\right) \delta 78.6,65.7,63.7,38.8,28.8,16.1,15.9$. IR (thin film): $v_{\max }$ 2982, 1269, $977 \mathrm{~cm}^{-1}$. MS (EI) m/z: (\%) 298 (M), 99, 65 (100). HRMS (APCI-LTQ Orbitrap) m/z: [M+H] ${ }^{+}$Calculated for $\mathrm{C}_{9} \mathrm{H}_{17} \mathrm{BrO}_{4} \mathrm{P}: 299.0042$; Found: 299.0042.

$\mathrm{TsO}\left(\mathrm{CH}_{2}\right)_{3}=\mathrm{Br}$

5-Bromopent-4-yn-1-yl 4-methylbenzenesulfonate (1r). This compound is known. ${ }^{10}$ 
${ }^{1} \mathrm{H}$ NMR (600 MHz, $\left.\mathrm{CDCl}_{3}\right) \delta 7.77(\mathrm{~d}, J=8.4 \mathrm{~Hz}, 2 \mathrm{H}), 7.34$ (d, $\left.J=7.8 \mathrm{~Hz}, 2 \mathrm{H}\right), 4.09$ $(\mathrm{t}, J=6.0 \mathrm{~Hz}, 2 \mathrm{H}), 2.43(\mathrm{~s}, 3 \mathrm{H}), 2.24(\mathrm{t}, J=7.2 \mathrm{~Hz}, 2 \mathrm{H}), 1.81(\mathrm{dt}, J=13.2 \mathrm{~Hz}, 6.0 \mathrm{~Hz}$, $2 \mathrm{H})$.

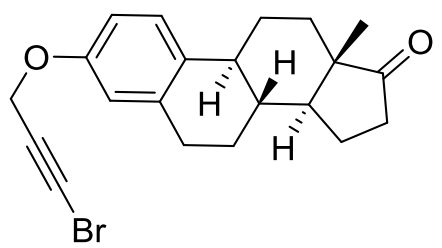

(8R,9S,13S,14S)-3-((3-bromoprop-2-yn-1-yl)oxy)-13-methyl-

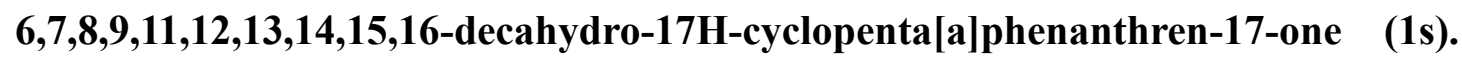
This compound is known. ${ }^{1}{ }^{1} \mathrm{H}$ NMR $\left(600 \mathrm{MHz}, \mathrm{CDCl}_{3}\right) \delta 7.22(\mathrm{~d}, J=8.6 \mathrm{~Hz}, 1 \mathrm{H})$, $6.77(\mathrm{dd}, J=8.6 \mathrm{~Hz}, 2.8 \mathrm{~Hz}, 1 \mathrm{H}), 6.69$ (d, 2.8 Hz, 1H), 4.67 (d, $J=2.4 \mathrm{~Hz}, 2 \mathrm{H}), 2.90$ (m, 2H), $2.51(\mathrm{~m}, 1 \mathrm{H}), 2.38(\mathrm{~m}, 1 \mathrm{H}), 2.25(\mathrm{~m}, 1 \mathrm{H}), 2.16(\mathrm{~m}, 1 \mathrm{H}), 2.04(\mathrm{~m}, 3 \mathrm{H}), 1.62$ (m, 2H), $1.52-1.39(\mathrm{~m}, 4 \mathrm{H}), 0.91(\mathrm{~s}, 3 \mathrm{H}) .{ }^{13} \mathrm{C}$ NMR $\left(150.8 \mathrm{MHz}, \mathrm{CDCl}_{3}\right) \delta 220.8$, $155.5,137.9,133.0,126.4,114.9,112.2,75.2,56.6,50.4,47.9,47.3,43.9,38.2,35.8$, 31.5, 29.6, 26.5, 25.8, 21.5, 13.8. IR (thin film): $v_{\max } 2928,2874,1040,2360,2223$, 1726, 1604, 1197, 1230, 1009 $\mathrm{cm}^{-1}$. HRMS (EI) m/z: $[\mathrm{M}+\mathrm{Na}]^{+}$Calculated for $\mathrm{C}_{21} \mathrm{H}_{23} \mathrm{O}_{2} \mathrm{BrNa}^{+}$: 409.0769; Found: 409.0774.

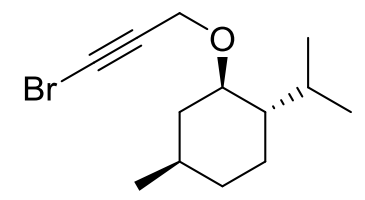

(1S,2R,4R)-2-((3-bromoprop-2-yn-1-yl)oxy)-1-isopropyl-4-methylcyclohexane (1t). ${ }^{1} \mathrm{H}$ NMR $\left(600 \mathrm{MHz}, \mathrm{CDCl}_{3}\right) \delta 4.20(\mathrm{~d}, \mathrm{dd}, J=15.8,1.2 \mathrm{~Hz} 1 \mathrm{H}), 4.16(\mathrm{~d}, \mathrm{~J}=15.8,1.2$ Hz, 1H), $3.23(\mathrm{td}, J=10.6,4.2 \mathrm{~Hz}, 1 \mathrm{H}), 2.20(\mathrm{~m}, 1 \mathrm{H}), 2.06(\mathrm{~m}, 1 \mathrm{H}), 1.62(\mathrm{ddq}, J=18.8$, 13.3, $3.4 \mathrm{~Hz}, 2 \mathrm{H}), 1.34$ (tdd, $J=12.2,6.5,3.3 \mathrm{~Hz}, 1 \mathrm{H}), 1.19(\mathrm{~m}, 1 \mathrm{H}), 0.99(\mathrm{~m}, 1 \mathrm{H})$, $0.91(\mathrm{~d}, J=6.5 \mathrm{~Hz}, 3 \mathrm{H}), 0.4(\mathrm{~m}, 2 \mathrm{H}), 0.88(\mathrm{~d}, \mathrm{~J}=7.0 \mathrm{~Hz}, 3 \mathrm{H}) .0 .82(\mathrm{~m}, 2 \mathrm{H}), 0.78(\mathrm{~d}, \mathrm{~J}=$ $6.9 \mathrm{~Hz}, 3 \mathrm{H}) .{ }^{13} \mathrm{C} \mathrm{NMR}\left(150.8 \mathrm{MHz}, \mathrm{CDCl}_{3}\right) \delta 78.0$, 77.2, 56.2 , 48.1 , $44.9,39.7$, $34.4,31.4,25.3,23.2,22.3,21.0,16.1$. IR (thin film): $v_{\max } 2924,2342$, 1685,1631,1604, 1559, 1384, 1083 $\mathrm{cm}^{-1}$. HRMS (EI) m/z: [M]+ Calculated for $\mathrm{C}_{13} \mathrm{H}_{22} \mathrm{O}_{2} \mathrm{Br}$ : 273.0849; Found: 273.0850.

\section{Representative results for optimization of copper-catalyzed perfluoroalkylation of} alkynyl bromides 1a

To a septum capped $25 \mathrm{~mL}$ of sealed tube were added $\mathrm{Cu}$ catalyst (0.01-10 mol\%), 
$\mathrm{Zn}\left(\mathrm{C}_{4} \mathrm{~F}_{9}\right)_{2}(\mathrm{DMPU})_{2}\left(1.0\right.$ equiv) under $\mathrm{N}_{2}$, followed by solvent $(2.0 \mathrm{~mL})$ with stirring. 1a $(0.2 \mathrm{mmol})$ was then added subsequently. The sealed tube was screw capped and heated to $40-100{ }^{\circ} \mathrm{C}$ (oil bath). After stirring for $12 \mathrm{~h}$, the reaction mixture was cooled to room temperature. The yield was determined by ${ }^{19} \mathrm{~F}$ NMR before working up. If necessary, the reaction mixture was diluted with ethyl acetate, washed with brine, dried over $\mathrm{Na}_{2} \mathrm{SO}_{4}$, filtered and concentrated. The residue was purified with silica gel chromatography (Petroleum ether $(100 \%))$ to provide pure product.

\section{General procedure for copper-catalyzed perfluoroalkylation of alkynyl bromides}

To a septum capped $25 \mathrm{~mL}$ of sealed tube were added $\mathrm{CuI}(0.5 \mathrm{~mol} \%)$, $\left.\mathrm{Zn}\left(\mathrm{R}_{\mathrm{F}}\right)_{2}(\mathrm{DMPU})_{2}(0.2 \mathrm{mmol})\right)$ under $\mathrm{N}_{2}$, followed by toluene $(2.0 \mathrm{~mL})$ with stirring. 1 $(0.2 \mathrm{mmol})$ was then added subsequently. The sealed tube was screw capped and heated to $80{ }^{\circ} \mathrm{C}$ (oil bath). After stirring for $12 \mathrm{~h}$, the reaction mixture was cooled to room temperature. The reaction mixture was diluted with ethyl acetate, washed with brine, dried over $\mathrm{Na}_{2} \mathrm{SO}_{4}$, filtered and concentrated. The residue was purified with silica gel chromatography to provide pure product.

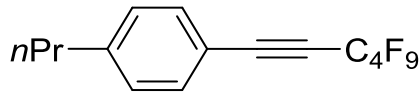

1-(perfluorohex-1-yn-1-yl)-4-propylbenzene (6a). This compound is known. ${ }^{11}$ The product (67 mg, 92\% yield) as a pale yellow oil was purified with silica gel chromatography (Petroleum ether (100\%)). ${ }^{1} \mathrm{H}$ NMR (600 MHz, $\left.\mathrm{CDCl}_{3}\right) \delta 7.47(\mathrm{~d}, J=$ $7.8 \mathrm{~Hz}, 2 \mathrm{H}), 7.21(\mathrm{~d}, J=7.8 \mathrm{~Hz}, 2 \mathrm{H}), 2.62(\mathrm{t}, J=7.2 \mathrm{~Hz}, 2 \mathrm{H}), 1.65(\mathrm{~m}, 2 \mathrm{H}), 0.94(\mathrm{t}, J$ $=7.2 \mathrm{~Hz}, 3 \mathrm{H}) .{ }^{19} \mathrm{~F}$ NMR $\left(564 \mathrm{MHz}, \mathrm{CDCl}_{3}\right) \delta-81.1(\mathrm{t}, J=9.0 \mathrm{~Hz}, 3 \mathrm{~F}),-97.1(\mathrm{~s}, 2 \mathrm{~F}),-$ $123.8(\mathrm{t}, J=5.6 \mathrm{~Hz}, 2 \mathrm{~F}),-125.5(\mathrm{q}, J=4.0 \mathrm{~Hz}, 2 \mathrm{~F})$.<smiles>CCCCCCC#Cc1ccc(C#CC(F)(F)F)cc1</smiles>

1-pentyl-4-(perfluorohex-1-yn-1-yl)benzene (6b). This compound is known. ${ }^{11}$ The product (69 mg, 88\% yield) as a pale yellow oil was purified with silica gel chromatography (Petroleum ether (100\%)). ${ }^{1} \mathrm{H}$ NMR (600 MHz, $\left.\mathrm{CDCl}_{3}\right) \delta 7.47(\mathrm{~d}, J=$ $7.8 \mathrm{~Hz}, 2 \mathrm{H}), 7.21(\mathrm{~d}, J=8.4 \mathrm{~Hz}, 2 \mathrm{H}), 2.64(\mathrm{t}, J=7.2 \mathrm{~Hz}, 2 \mathrm{H}), 1.62(\mathrm{~m}, 2 \mathrm{H}), 1.32(\mathrm{~m}$, $4 \mathrm{H}), 0.90(\mathrm{t}, J=6.6 \mathrm{~Hz}, 3 \mathrm{H}) .{ }^{19} \mathrm{~F} \mathrm{NMR}\left(564 \mathrm{MHz}, \mathrm{CDCl}_{3}\right) \delta-81.1(\mathrm{t}, J=9.6 \mathrm{~Hz}, 3 \mathrm{~F})$, $-97.1(\mathrm{~d}, J=2.3 \mathrm{~Hz}, 2 \mathrm{~F}),-123.5(\mathrm{~m}, 2 \mathrm{~F}),-125.5(\mathrm{~m}, 2 \mathrm{~F})$

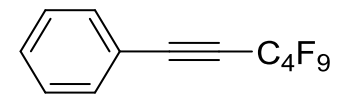


(perfluorohex-1-yn-1-yl)benzene (6c). This compound is known. ${ }^{11}$ (53 mg, 83\% yield) as a pale yellow oil was purified with silica gel chromatography (Petroleum ether (100\%)). ${ }^{1} \mathrm{H}$ NMR (600 MHz, $\left.\mathrm{CDCl}_{3}\right) \delta 7.57(\mathrm{~d}, J=7.2 \mathrm{~Hz}, 2 \mathrm{H}), 7.50(\mathrm{t}, J=7.8 \mathrm{~Hz}$, 2H), $7.41(\mathrm{t}, J=7.8 \mathrm{~Hz}, 2 \mathrm{H}) .{ }^{19} \mathrm{~F} \mathrm{NMR}\left(564 \mathrm{MHz}, \mathrm{CDCl}_{3}\right) \delta-81.7(\mathrm{~s}, 3 \mathrm{~F}),-98.0(\mathrm{~s}, 2 \mathrm{~F})$, $-124.1(\mathrm{~s}, 2 \mathrm{~F}),-126.1(\mathrm{~s}, 2 \mathrm{~F})$.

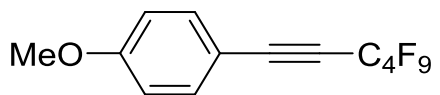

1-methoxy-4-(perfluorohex-1-yn-1-yl)benzene (6d). This compound is known. ${ }^{11}$ The product (63 mg, 90\% yield) as a pale yellow oil was purified with silica gel chromatography (Petroleum ether (100\%)). ${ }^{1} \mathrm{H}$ NMR $\left(600 \mathrm{MHz}, \mathrm{CDCl}_{3}\right) \delta 7.50(\mathrm{~d}, J=$ $9.0 \mathrm{~Hz}, 2 \mathrm{H}), 6.90(\mathrm{~d}, J=9.0 \mathrm{~Hz}, 2 \mathrm{H}), 3.84(\mathrm{~s}, 3 \mathrm{H}) .{ }^{19} \mathrm{~F} \mathrm{NMR}\left(564 \mathrm{MHz}, \mathrm{CDCl}_{3}\right) \delta-$ $81.6(\mathrm{t}, J=9.6 \mathrm{~Hz}, 3 \mathrm{~F}),-97.4(\mathrm{~d}, J=5.6 \mathrm{~Hz}, 2 \mathrm{~F}),-124.0(\mathrm{~s}, 2 \mathrm{~F}),-126.1(\mathrm{~d}, J=6.8 \mathrm{~Hz}$, $2 \mathrm{~F})$.

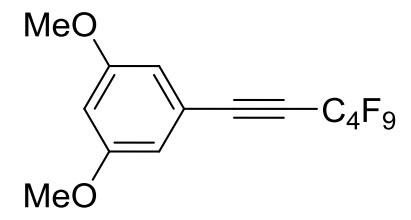

1,3-dimethoxy-5-(perfluorohex-1-yn-1-yl)benzene (6e). This compound is known. ${ }^{11}$ The product (57 mg, 75\% yield) as a pale yellow oil was purified with silica gel chromatography (Petroleum ether / Ethyl ether $=40: 1) .{ }^{1} \mathrm{H} \mathrm{NMR}\left(600 \mathrm{MHz}, \mathrm{CDCl}_{3}\right) \delta$ $6.69(\mathrm{~s}, 2 \mathrm{H}), 6.58(\mathrm{~s}, 2 \mathrm{H}), 3.80(\mathrm{~s}, 6 \mathrm{H}) .{ }^{19} \mathrm{~F} \mathrm{NMR}\left(564 \mathrm{MHz}, \mathrm{CDCl}_{3}\right) \delta-81.1(\mathrm{t}, J=9.0$ $\mathrm{Hz}, 3 \mathrm{~F}),-97.5$ (s, 2F), $-123.4(\mathrm{t}, J=4.5 \mathrm{~Hz}, 2 \mathrm{~F}),-125.5$ (q, $J=4.5 \mathrm{~Hz}, 2 \mathrm{~F})$.

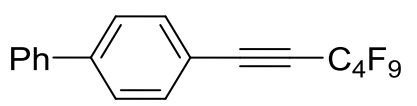

4-(perfluorohex-1-yn-1-yl)-1,1'-biphenyl (6f). This compound is known. ${ }^{11}$ The product (68 mg, $86 \%$ yield) as a white solid was purified with silica gel chromatography (Petroleum ether (100\%)). ${ }^{1} \mathrm{H}$ NMR $\left(600 \mathrm{MHz}, \mathrm{CDCl}_{3}\right) \delta 7.64(\mathrm{~s}, J=9.0 \mathrm{~Hz}, 4 \mathrm{H}), 7.61$ $(\mathrm{d}, J=7.2 \mathrm{~Hz}, 2 \mathrm{H}), 7.49$ (t, $J=7.2 \mathrm{~Hz}, 3 \mathrm{H}), 7.42(\mathrm{t}, J=7.8 \mathrm{~Hz}, 1 \mathrm{H}) .{ }^{19} \mathrm{~F}$ NMR $(564$ $\left.\mathrm{MHz}, \mathrm{CDCl}_{3}\right) \delta-81.0(\mathrm{~d}, J=7.3 \mathrm{~Hz}, 3 \mathrm{~F}),-97.3(\mathrm{~s}, 2 \mathrm{~F}),-123.4(\mathrm{~s}, 2 \mathrm{~F}),-125.5(\mathrm{~s}, 2 \mathrm{~F})$.

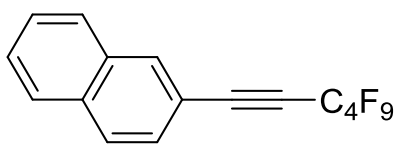

3-(Perfluorohex-1-yn-1-yl)pyridine (6g). This compound is known. ${ }^{11}$ The product (55 mg, $85 \%$ yield) as a pale yellow oil was purified with silica gel chromatography (Petroleum ether (100\%). ${ }^{1} \mathrm{H}$ NMR (600 MHz, $\left.\mathrm{CDCl}_{3}\right) \quad 8.13(\mathrm{~s}, 3 \mathrm{H} \mathrm{1H}), 7.85(\mathrm{~m}, 3 \mathrm{H})$, 
$7.57(\mathrm{~m}, 3 \mathrm{H}) .{ }^{19} \mathrm{~F} \mathrm{NMR}\left(564 \mathrm{MHz}, \mathrm{CDCl}_{3}\right) \quad-81.0(\mathrm{~m}, 3 \mathrm{~F}),-97.2(\mathrm{~s}, 2 \mathrm{~F}),-123.3(\mathrm{~s}, 2 \mathrm{~F})$, $-125.4(\mathrm{~s}, 2 \mathrm{~F})$.

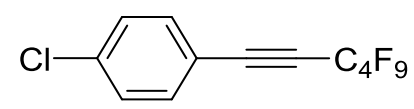

1-chloro-4-(perfluorohex-1-yn-1-yl)benzene (6h). This compound is known. ${ }^{11}$ The product (52 mg, 73\% yield) as a pale yellow oil was purified with silica gel chromatography (Petroleum ether (100\%)). ${ }^{1} \mathrm{H}$ NMR (600 MHz, $\left.\mathrm{CDCl}_{3}\right) \delta 7.50(\mathrm{~d}, J=$ $8.4 \mathrm{~Hz}, 2 \mathrm{H}), 7.43(\mathrm{~d}, J=9.0 \mathrm{~Hz}, 2 \mathrm{H}) .{ }^{19} \mathrm{~F} \mathrm{NMR}\left(564 \mathrm{MHz}, \mathrm{CDCl}_{3}\right) \delta-76.8(\mathrm{t}, J=9.6$ $\mathrm{Hz}, 3 \mathrm{~F}),-93.5$ (s, 2F), -119.2 (s, 2F), -121.3 (s, 2F).

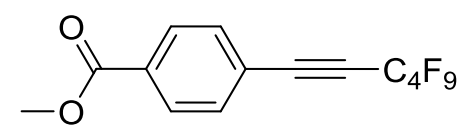

Methyl 4-(perfluorohex-1-yn-1-yl)benzoate (6i). This compound is known. ${ }^{11}$ The product (68 mg, 90\% yield) as a white solid was purified with silica gel chromatography (Petroleum ether / Ethyl acetate $=20: 1) .{ }^{1} \mathrm{H}$ NMR $\left(600 \mathrm{MHz}, \mathrm{CDCl}_{3}\right) \delta 8.07(\mathrm{~d}, J=$ $8.4 \mathrm{~Hz}, 2 \mathrm{H}), 7.64(\mathrm{~d}, J=8.4 \mathrm{~Hz}, 2 \mathrm{H}), 3.95(\mathrm{~s}, 3 \mathrm{H}) .{ }^{19} \mathrm{~F}$ NMR $\left(564 \mathrm{MHz}, \mathrm{CDCl}_{3}\right) \delta-80.0$ (s, 3F), $-98.0(\mathrm{~s}, 2 \mathrm{~F}),-123.4(\mathrm{~s}, 2 \mathrm{~F}),-125.5(\mathrm{~s}, 2 \mathrm{~F})$.

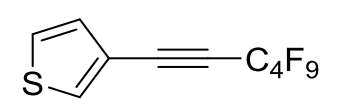

3-(Perfluorohex-1-yn-1-yl)thiophene(6j). This compound is known. ${ }^{11}$ The product (56 mg, $87 \%$ yield) as a pale yellow oil was purified with silica gel chromatography (Petroleum ether (100\%). ${ }^{1} \mathrm{H}$ NMR $\left(600 \mathrm{MHz}, \mathrm{CDCl}_{3}\right) \delta 7.73(\mathrm{~d}, J=1.8 \mathrm{~Hz}, 1 \mathrm{H}), 7.34$ (m, 1H), $7.20(\mathrm{~d}, J=4.8 \mathrm{~Hz}, 1 \mathrm{H}) .{ }^{19} \mathrm{~F}$ NMR $\left(564 \mathrm{MHz}, \mathrm{CDCl}_{3}\right) \delta-81.1(\mathrm{~m}, 3 \mathrm{~F}),-97.3$ $(\mathrm{s}, 2 \mathrm{~F}),-123.4(\mathrm{~m}, 2 \mathrm{~F}),-125.5(\mathrm{~m}, 2 \mathrm{~F})$.

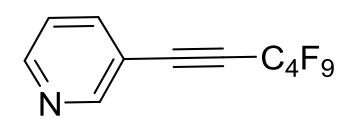

3-(Perfluorohex-1-yn-1-yl)pyridine (6k). The product (44 mg, 68\% yield) as a pale yellow oil was purified with silica gel chromatography (Petroleum ether $(100 \%) .{ }^{1} \mathrm{H}$ NMR $\left(600 \mathrm{MHz}, \mathrm{CDCl}_{3}\right) \delta 8.77(\mathrm{~s}, 1 \mathrm{H}), 8.68(\mathrm{~d}, J=4.8 \mathrm{~Hz}, 1 \mathrm{H}), 7.84(\mathrm{~d}, J=7.8 \mathrm{~Hz}$, 1H), $7.33(\mathrm{~m}, 1 \mathrm{H}) .{ }^{13} \mathrm{C} \mathrm{NMR}\left(150.8 \mathrm{MHz}, \mathrm{CDCl}_{3}\right) \delta 152.8(\mathrm{t}, J=2.4 \mathrm{~Hz}), 151.2,139.4$, 132.1, 118.4-116.1 [m, $\left.-\left(\mathrm{CF}_{2}\right) \mathrm{CF}_{3}\right], 115.8(\mathrm{t}, J=2.9 \mathrm{~Hz}), 110.2-105.4$ [m, $\left.-\left(\mathrm{CF}_{2}\right) \mathrm{CF}_{3}\right]$, $88.7(\mathrm{t}, J=6.3 \mathrm{~Hz}), 77.6(\mathrm{t}, J=36.3 \mathrm{~Hz}) .{ }^{19} \mathrm{~F} \mathrm{NMR}\left(564 \mathrm{MHz}, \mathrm{CDCl}_{3}\right) \delta-85.9(\mathrm{~m}, 3 \mathrm{~F})$, -103.1 (s, 2F), -128.2 (s, 2F), -130.3(s, 2F). IR (thin film): $v_{\max } 2925,1237,1135 \mathrm{~cm}^{-1}$. MS (EI) m/z: (\%) 321 (M), 152 (100), 99. HRMS (APCI-LTQ Orbitrap) m/z: [M+H] ${ }^{+}$ Calculated for $\mathrm{C}_{11} \mathrm{H}_{5} \mathrm{~F}_{9} \mathrm{~N}$ : 322.0273; Found: 322.0273 . 
$\mathrm{H}_{3} \mathrm{C}\left(\mathrm{CH}_{2}\right)_{9}=\mathrm{C}_{4} \mathrm{~F}_{9}$

1,1,1,2,2,3,3,4,4-Nonafluorohexadec-5-yne (61). This compound is known. ${ }^{11}$ The product (54 mg, 71\% yield) as a colorless oil was purified with silica gel chromatography (Petroleum ether (100\%). ${ }^{1} \mathrm{H}$ NMR $\left(600 \mathrm{MHz}, \mathrm{CDCl}_{3}\right) \delta 2.35(\mathrm{dt}, J=$ $12.6 \mathrm{~Hz}, 6.6 \mathrm{~Hz}, 2 \mathrm{H}), 1.58(\mathrm{dt}, J=15.0 \mathrm{~Hz}, 7.2 \mathrm{~Hz}, 2 \mathrm{H}), 1.39(\mathrm{~m}, 2 \mathrm{H}), 1.28(\mathrm{~m}, 13 \mathrm{H})$, $0.88(\mathrm{t}, J=6.6 \mathrm{~Hz}, 3 \mathrm{H}) .{ }^{19} \mathrm{~F} \mathrm{NMR}\left(564 \mathrm{MHz}, \mathrm{CDCl}_{3}\right) \delta-81.2(\mathrm{~s}, 3 \mathrm{~F}),-96.5(\mathrm{~s}, 2 \mathrm{~F}),-123.6$ (s, 2F), -125.5 (s,2F).

$\mathrm{TBSO}\left(\mathrm{CH}_{2}\right)_{3}=\mathrm{C}_{4} \mathrm{~F}_{9}$

tert-Butyldimethyl((6,6,7,7,8,8,9,9,9-nonafluoronon-4-yn-1-yl)oxy)silane $\quad(6 \mathrm{~m})$. The product (52 mg, 63\% yield) as a colorless oil was purified with silica gel chromatography (Petroleum ether (100\%). ${ }^{1} \mathrm{H}$ NMR $\left(600 \mathrm{MHz}, \mathrm{CDCl}_{3}\right) \delta 3.69(\mathrm{t}, J=$ $5.4 \mathrm{~Hz}, 2 \mathrm{H}), 2.47$ (dt, $J=12.6 \mathrm{~Hz}, 6.6 \mathrm{~Hz}, 2 \mathrm{H}), 1.78$ (dt, $J=12.6 \mathrm{~Hz}, 6.0 \mathrm{~Hz}, 2 \mathrm{H}), 0.89$ (s, 9H), 0.05(s, 6H). ${ }^{13} \mathrm{C}$ NMR (150.8 MHz, $\left.\mathrm{CDCl}_{3}\right) \delta 122.0-104.0\left[\mathrm{~m},-\left(\mathrm{CF}_{2}\right) \mathrm{CF}_{3}\right], 95.0$ (t, $J=9.2 \mathrm{~Hz}), 67.3(\mathrm{t}, J=53.7 \mathrm{~Hz}), 60.7,30.2,29.7,25.7,18.2,14.8,5.7 .{ }^{19} \mathrm{~F}$ NMR $\left(564 \mathrm{MHz}, \mathrm{CDCl}_{3}\right) \delta-81.2(\mathrm{~S}, 3 \mathrm{~F}),-96.6(\mathrm{~s}, 2 \mathrm{~F}),-123.6(\mathrm{~s}, 2 \mathrm{~F}),-125.6(\mathrm{~s}, 2 \mathrm{~F})$. IR (thin film): $v_{\max } 2982,1269,977 \mathrm{~cm}^{-1}$. MS (EI) m/z: (\%) 416 (M), 146, 77 (100). HRMS (EI) $\mathrm{m} / \mathrm{z}:[\mathrm{M}]^{+}$Calculated for $\mathrm{C}_{15} \mathrm{H}_{21} \mathrm{~F}_{9} \mathrm{OSi}$ : 416.1218; Found: 416.1215.

$\mathrm{AcO}\left(\mathrm{CH}_{2}\right)_{3}=\mathrm{C}_{4} \mathrm{~F}_{9}$

$\mathbf{6 , 6 , 7 , 7 , 8 , 8 , 9 , 9 , 9 - N o n a f l u o r o n o n - 4 - y n - 1 - y l ~ a c e t a t e ~ ( 6 n ) . ~ T h e ~ p r o d u c t ~ ( 5 4 ~ m g , ~ 7 9 \% ~}$ yield) as a pale yellow oil was purified with silica gel chromatography (Petroleum ether / Ethyl acetate $=20: 1) .{ }^{1} \mathrm{H}$ NMR $\left(600 \mathrm{MHz}, \mathrm{CDCl}_{3}\right) \delta 4.15(\mathrm{t}, J=6.6 \mathrm{~Hz}, 2 \mathrm{H}), 2.48(\mathrm{dt}$, $J=12.6 \mathrm{~Hz}, 6.0 \mathrm{~Hz}, 2 \mathrm{H}), 2.06(\mathrm{~s}, 3 \mathrm{H}), 1.93(\mathrm{dt}, J=13.2 \mathrm{~Hz}, 6.0 \mathrm{~Hz}, 2 \mathrm{H}) .{ }^{13} \mathrm{C} \mathrm{NMR}(150.8$ $\left.\mathrm{MHz}, \mathrm{CDCl}_{3}\right) \delta 170.8,118.2-104.8\left[\mathrm{~m},-\left(\mathrm{CF}_{2}\right) \mathrm{CF}_{3}\right], 93.7(\mathrm{t}, J=6.0 \mathrm{~Hz}), 67.7(\mathrm{t}, J=$ 36.0 Hz), 62.4, 26.4, 20.6, 15.4. ${ }^{19} \mathrm{~F}$ NMR (564 MHz, $\left.\mathrm{CDCl}_{3}\right) \delta-81.2(\mathrm{~s}, 3 \mathrm{~F}),-96.5(\mathrm{~s}$, 2F), -123.6 (s, 2F), -125.5 (s, 2F). IR (thin film): $v_{\max } 2360,1239,671 \mathrm{~cm}^{-1}$. MS (EI) m/z: (\%) 344 (M), 155, 91 (100). HRMS (EI) m/z: [M] Calculated for $\mathrm{C}_{11} \mathrm{H}_{9} \mathrm{~F}_{9} \mathrm{O}_{2}$ : 344.0459; Found: 344.0455.

$\mathrm{NC}-\mathrm{CO}_{2}\left(\mathrm{CH}_{2}\right)_{3}=\mathrm{C}_{4} \mathrm{~F}_{9}$

$\mathbf{6 , 6 , 7 , 7 , 8 , 8 , 9 , 9 , 9 - N o n a f l u o r o n o n - 4 - y n - 1 - y l ~ 4 - c y a n o b e n z o a t e ~ ( 6 0 ) . ~ T h e ~ p r o d u c t ~ ( 6 4 ~}$ mg, 74\% yield) as a pale yellow oil was purified with silica gel chromatography (Petroleum ether $/$ Ethyl acetate $=20: 1) .{ }^{1} \mathrm{H}$ NMR $\left(600 \mathrm{MHz}, \mathrm{CDCl}_{3}\right) \delta 8.13(\mathrm{~d}, J=7.2$ $\mathrm{Hz}, 2 \mathrm{H}), 7.74(\mathrm{~d}, J=8.4 \mathrm{~Hz}, 2 \mathrm{H}), 4.45(\mathrm{t}, J=6.0 \mathrm{~Hz}, 2 \mathrm{H}), 2.57$ (dt, $J=12.0 \mathrm{~Hz}, 6.6 \mathrm{~Hz}$, 
2H), $2.10(\mathrm{dt}, J=13.2 \mathrm{~Hz}, 6.6 \mathrm{~Hz}, 2 \mathrm{H}) .{ }^{13} \mathrm{C} \mathrm{NMR}\left(150.8 \mathrm{MHz}, \mathrm{CDCl}_{3}\right) \delta$ 164.7, 133.6, 132.2, 130.1, 120.3-117.0 [m, - $\left.\left(\mathrm{CF}_{2}\right) \mathrm{CF}_{3}\right], 117.8,116.6,116.5-104.8$ [m, $\left.-\left(\mathrm{CF}_{2}\right) \mathrm{CF}_{3}\right]$, $93.4(\mathrm{t}, J=6.0 \mathrm{~Hz}), 68.0(\mathrm{t}, J=35.9 \mathrm{~Hz}), 63.8,26.4,15.6(\mathrm{t}, J=2.4 \mathrm{~Hz}) .{ }^{19} \mathrm{~F} \mathrm{NMR}(564$ $\left.\mathrm{MHz} \mathrm{CDCl}_{3}\right) \delta-81.2(\mathrm{~s}, 3 \mathrm{~F}),-97.1(\mathrm{~s}, 2 \mathrm{~F}),-123.5(\mathrm{~s}, 2 \mathrm{~F}),-125.5$ (s, 2F). IR (thin film): $v_{\max } 2258,1735,861 \mathrm{~cm}^{-1}$.MS (EI) m/z: (\%) 431 (M), 130 (100), 102. HRMS (APCILTQ Orbitrap) m/z: [M+H] $]^{+}$Calculated for $\mathrm{C}_{17} \mathrm{H}_{11} \mathrm{~F}_{9} \mathrm{NO}_{2}$ : 432.0641 ; Found: 432.0641.<smiles>O=C(OCCCC#CC(F)(F)F)c1ccc([N+](=O)[O-])cc1</smiles>

6,6,7,7,8,8,9,9,9-Nonafluoronon-4-yn-1-yl 4-nitrobenzoate (6p). The product (62 mg, $68 \%$ yield) as a pale yellow oil was purified with silica gel chromatography (Petroleum ether / Ethyl acetate =15:1). ${ }^{1} \mathrm{H}$ NMR $\left(600 \mathrm{MHz}, \mathrm{CDCl}_{3}\right) \delta 8.29(\mathrm{~d}, J=8.4 \mathrm{~Hz}, 2 \mathrm{H})$, $8.20(\mathrm{~d}, J=9.0 \mathrm{~Hz}, 2 \mathrm{H}), 4.47(\mathrm{t}, J=6.6 \mathrm{~Hz}, 2 \mathrm{H}), 2.59$ (dt, $J=12 \mathrm{~Hz}, 6.0 \mathrm{~Hz}, 2 \mathrm{H}), 2.12$ $(\mathrm{dt}, J=12.6 \mathrm{~Hz}, 6.6 \mathrm{~Hz}, 2 \mathrm{H}) .{ }^{13} \mathrm{C} \mathrm{NMR}\left(150.8 \mathrm{MHz}, \mathrm{CDCl}_{3}\right) \delta 164.4,150.6,135.2,130.7$, 123.5, 118.4-105.1 [m, - $\left.\left(\mathrm{CF}_{2}\right) \mathrm{CF}_{3}\right], 93.3(\mathrm{t}, J=6.0 \mathrm{~Hz}), 68.0(\mathrm{t}, J=35.9 \mathrm{~Hz}), 63.8,26.4$, $15.6(\mathrm{t}, J=2.4 \mathrm{~Hz}) .{ }^{19} \mathrm{~F} \mathrm{NMR}\left(564 \mathrm{MHz}, \mathrm{CDCl}_{3}\right) \delta-81.1(\mathrm{t}, J=9.0 \mathrm{~Hz}, 3 \mathrm{~F}),-97.1(\mathrm{~s}$, 2F), -123.5 (s, 2F), -125.5 (s, 2F). IR (thin film): $v_{\max } 2258,1533,719 \mathrm{~cm}^{-1}$.MS (EI) m/z: (\%) 451 (M), 150 (100), 115. HRMS (APCI-LTQ Orbitrap) m/z: [M+H] $]^{+}$ Calculated for $\mathrm{C}_{16} \mathrm{H}_{11} \mathrm{~F}_{9} \mathrm{NO}_{4}$ : 452.0539; Found: 452.0540 .<smiles>CCO[Po](=O)OCCCC#CC(F)(F)F</smiles>

Diethyl (6,6,7,7,8,8,9,9,9-nonafluoronon-4-yn-1-yl) phosphate (6q). The product (70 $\mathrm{mg}, 80 \%$ yield) as a pale yellow oil was purified with silica gel chromatography (dichloromethane / methyl alcohol =20:1). ${ }^{1} \mathrm{H} \mathrm{NMR}\left(600 \mathrm{MHz}, \mathrm{CDCl}_{3}\right) \delta 4.10(\mathrm{dt}, J=$ $14.4 \mathrm{~Hz}, 7.2 \mathrm{~Hz}, 6 \mathrm{H}), 2.17$ (dt, $J=12.6 \mathrm{~Hz}, 6.0 \mathrm{~Hz}, 2 \mathrm{H}), 1.94$ (dt, $J=13.2 \mathrm{~Hz}, 6.6 \mathrm{~Hz}$ ), $2.06(\mathrm{t}, J=7.2 \mathrm{~Hz}, 3 \mathrm{H}) .{ }^{13} \mathrm{C} \mathrm{NMR}\left(150.8 \mathrm{MHz}, \mathrm{CDCl}_{3}\right) \delta 119.1-106.4\left[\mathrm{~m},-\left(\mathrm{CF}_{2}\right) \mathrm{CF}_{3}\right]$, $93.7(\mathrm{t}, J=8.9 \mathrm{~Hz}), 68.0(\mathrm{t}, J=53.8 \mathrm{~Hz}), 65.4,64.0,28.1,16.2,15.0(\mathrm{t}, J=3.6 \mathrm{~Hz}) .{ }^{19} \mathrm{~F}$ $\operatorname{NMR}\left(564 \mathrm{MHz}, \mathrm{CDCl}_{3}\right) \delta-81.1(\mathrm{t}, J=9.6 \mathrm{~Hz}, 3 \mathrm{~F}),-96.9(\mathrm{~s}, 2 \mathrm{~F}),-123.5(\mathrm{~s}, 2 \mathrm{~F}),-125.5$ (m, 2F). IR (thin film): $v_{\max }$ 2988, 1239, $746 \mathrm{~cm}^{-1}$. MS (EI) m/z: (\%) 438 (M), 217, 99 (100). HRMS (APCI-LTQ Orbitrap) $\mathrm{m} / \mathrm{z}$ : $[\mathrm{M}+\mathrm{H}]^{+}$Calculated for $\mathrm{C}_{13} \mathrm{H}_{17} \mathrm{~F}_{9} \mathrm{O}_{4} \mathrm{P}$ : 439.0715; Found: 439.0715.

$\mathrm{TsO}\left(\mathrm{CH}_{2}\right)_{3}=\mathrm{C}_{4} \mathrm{~F}_{9}$

6,6,7,7,8,8,9,9,9-Nonafluoronon-4-yn-1-yl 4-methylbenzenesulfonate (6r). The 
product (77 $\mathrm{mg}, 85 \%$ yield) as a pale yellow oil was purified with silica gel chromatography (Petroleum ether / Ethyl acetate $=10: 1) .{ }^{1} \mathrm{H}$ NMR $\left(600 \mathrm{MHz}, \mathrm{CDCl}_{3}\right)$ $\delta 7.76(\mathrm{~d}, J=8.4 \mathrm{~Hz}, 2 \mathrm{H}), 7.33(\mathrm{~d}, J=7.8 \mathrm{~Hz}, 2 \mathrm{H}), 4.08(\mathrm{t}, J=6.0 \mathrm{~Hz}, 2 \mathrm{H}), 2.44(\mathrm{~m}$, $5 \mathrm{H}), 1.92(\mathrm{dt}, J=12.6 \mathrm{~Hz}, 6.6 \mathrm{~Hz}, 2 \mathrm{H}) .{ }^{13} \mathrm{C} \mathrm{NMR}\left(150.8 \mathrm{MHz}, \mathrm{CDCl}_{3}\right) \delta 145.1,132.6$, 130.0, 127.8, 118.4-104.2 [m, - $\left.\left(\mathrm{CF}_{2}\right) \mathrm{CF}_{3}\right], 92.8$ (t, $\left.J=9.2 \mathrm{~Hz}\right), 68.2$ (t, $\left.J=54.0 \mathrm{~Hz}\right)$, 67.9, 26.8, 21.5, 14.8. ${ }^{19} \mathrm{~F}$ NMR (564 MHz, $\left.\mathrm{CDCl}_{3}\right) \delta-81.1$ (m, 3F), -97.2 (m, 2F), 123.5 (m, 2F),-125.5 (m, 2F). IR (thin film): $v_{\max } 2262,1242,554 \mathrm{~cm}^{-1}$. MS (EI) m/z: (\%) $556(\mathrm{M}), 155,91$ (100). HRMS (EI) m/z: [M+Na] ${ }^{+}$Calculated for $\mathrm{C}_{16} \mathrm{H}_{13} \mathrm{~F}_{9} \mathrm{O}_{3} \mathrm{SNa}$ : 479.0334; Found: 479.0336.

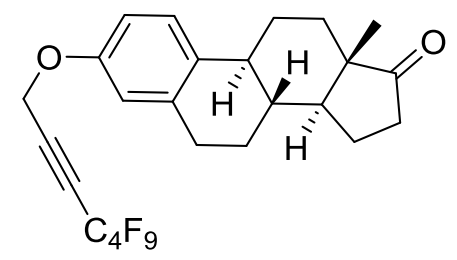

(8R,9S,13S,14S)-13-methyl-3-((4,4,5,5,6,6,7,7,7-nonafluorohept-2-yn-1-yl)oxy)-

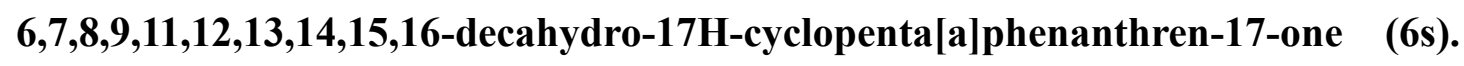
The product (95 mg, 90\% yield) as a colorless oil was purified with silica gel chromatography (Petroleum ether / Ethyl acetate $=10: 1) .{ }^{1} \mathrm{H} \mathrm{NMR}\left(600 \mathrm{MHz}, \mathrm{CDCl}_{3}\right)$ $\delta 7.24(\mathrm{dd}, J=8.8,1.0 \mathrm{~Hz}, 1 \mathrm{H}), 6.76(\mathrm{dd}, J=8.6,2.9 \mathrm{~Hz}, 1 \mathrm{H}), 6.70(\mathrm{~d}, J=2.8 \mathrm{~Hz}, 1 \mathrm{H})$, $4.79(\mathrm{t}, J=4.5 \mathrm{~Hz}, 2 \mathrm{H}), 2.96-2.85(\mathrm{~m}, 2 \mathrm{H}), 2.55-2.47(\mathrm{~m}, 1 \mathrm{H}), 2.44-2.35(\mathrm{~m}, 1 \mathrm{H})$, $2.26(\mathrm{td}, J=10.8,4.3 \mathrm{~Hz}, 1 \mathrm{H}), 2.15(\mathrm{dt}, J=19.1 \mathrm{~Hz}, 9.0 \mathrm{~Hz}, 1 \mathrm{H}), 2.10-1.93(\mathrm{~m}, 3 \mathrm{H})$, $1.62(\mathrm{~m}, 2 \mathrm{H}), 1.52-1.40(\mathrm{~m}, 4 \mathrm{H}), 0.92(\mathrm{~s}, 3 \mathrm{H}) .{ }^{19} \mathrm{~F} \mathrm{NMR}\left(564 \mathrm{MHz}, \mathrm{CDCl}_{3}\right) \delta-81.09$ (t, $J=9.7 \mathrm{~Hz}, 3 \mathrm{~F}),-98.56--98.87$ (m, 2F), -123.41 (tq, $J=10.5 \mathrm{~Hz}, 5.2 \mathrm{~Hz}, 2 \mathrm{~F}),-$ $125.40--125.71(\mathrm{~m}, 2 \mathrm{~F}) .{ }^{13} \mathrm{C} \mathrm{NMR}\left(151 \mathrm{MHz}, \mathrm{CDCl}_{3}\right) \delta 220.8,155.1,138.1,133.8$, $126.5,118.2,116.3,115.0,114.4,112.4,108.3,88.3,72.9,55.3,50.4,48.0,44.0,38.2$, $35.8,31.5,29.6,26.4,25.8,21.6,13.8$. IR (thin film): vmax 2924, 2360, 1740, 1684, 1631, 1540, 1236, 1138, 1057 $\mathrm{cm}^{-1}$. HRMS (EI) $\mathrm{m} / \mathrm{z}:[\mathrm{M}+\mathrm{Na}]^{+}$Calculated for $\mathrm{C}_{25} \mathrm{H}_{23} \mathrm{O}_{2} \mathrm{~F}_{9} \mathrm{Na}$ : 549.1447; Found: 549.1445.<smiles>CC1CCC(C(C)C)C(OCC#CC(F)(F)F)C1</smiles>

(1S,2R,4R)-1-isopropyl-4-methyl-2-((4,4,5,5,6,6,7,7,7-nonafluorohept-2-yn-1-

yl)oxy)cyclohexane (6t). The product ( $75 \mathrm{mg}, 91 \%$ yield) as a colorless oil was purified with silica gel chromatography (Petroleum ether / Ethyl acetate $=100: 1$ ). ${ }^{1} \mathrm{H}$ NMR $\left(600 \mathrm{MHz}, \mathrm{CDCl}_{3}\right) \delta 4.33(\mathrm{~m}, 2 \mathrm{H}), 3.24(\mathrm{td}, \mathrm{J}=10.6,4.2 \mathrm{~Hz}, 1 \mathrm{H}), 2.17(\mathrm{pd}, J=7.0,2.6$ 
$\mathrm{Hz}, 1 \mathrm{H}), 2.11(\mathrm{dtd}, J=12.2,3.8,1.9 \mathrm{~Hz}, 1 \mathrm{H}), 1.65$ (dddd, $J=16.5,13.1,6.2,3.4 \mathrm{~Hz}$, 2H), $1.36(\mathrm{ttd}, J=15.0,6.5,3.2 \mathrm{~Hz}, 1 \mathrm{H}), 1.23(\mathrm{tt}, J=10.3,3.0 \mathrm{~Hz}, 1 \mathrm{H}), 1.05-0.97$ (m, $1 \mathrm{H}), 0.89(\mathrm{~d}, J=6.5 \mathrm{~Hz}, 3 \mathrm{H}), 0.86(\mathrm{~d}, J=7.0 \mathrm{~Hz} 3 \mathrm{H}), 0.88-0.80(\mathrm{~m}, 2 \mathrm{H}), 0.78(\mathrm{~d}, \mathrm{~J}$ $=6.9 \mathrm{~Hz}, 3 \mathrm{H}) .{ }^{19} \mathrm{~F}$ NMR $\left(564 \mathrm{MHz}, \mathrm{CDCl}_{3}\right) \delta-81.21(\mathrm{t}, J=9.7 \mathrm{~Hz}, 3 \mathrm{~F}),-98.10(\mathrm{~m}, 2 \mathrm{~F})$, $-123.41(\mathrm{tq}, J=10.5 \mathrm{~Hz}, 5.2 \mathrm{~Hz}, 2 \mathrm{~F}),-125.65$ (m, 2F). ${ }^{13} \mathrm{C} \mathrm{NMR}\left(150.8 \mathrm{MHz}, \mathrm{CDCl}_{3}\right)$ $\delta 118.0,116.3,108.4,106.8,48.1,39.6,34.3,31.4,25.4,23.2,22.1,20.8,16.0$. IR (thin film): $v_{\max } 2924,2853,2360,1685,1631,1604,1559,1540,1384,1236,1137,865 \mathrm{~cm}^{-1}$. HRMS (EI) m/z: [M+H]+ Calculated for $\mathrm{C}_{17} \mathrm{H}_{22} \mathrm{OF} 9$ : 413.1522 ; Found: 413.1527.<smiles>CCCCCCC(F)(F)C(F)C#Cc1ccc(CCCC)cc1</smiles>

1-Pentyl-4-(perfluoropent-1-yn-1-yl)benzene (5b). The product (54 mg, 80\% yield) as a pale yellow oil was purified with silica gel chromatography (Petroleum ether (100\%). ${ }^{1} \mathrm{H} \mathrm{NMR}\left(600 \mathrm{MHz}, \mathrm{CDCl}_{3}\right) \delta 7.46(\mathrm{~d}, J=8.4 \mathrm{~Hz}, 2 \mathrm{H}), 7.20(\mathrm{~d}, J=7.8 \mathrm{~Hz}, 2 \mathrm{H})$, $2.63(\mathrm{t}, J=7.8 \mathrm{~Hz}, 2 \mathrm{H}), 1.60(\mathrm{dt}, J=15.0 \mathrm{~Hz}, 7.8 \mathrm{~Hz}, 2 \mathrm{H}), 1.31(\mathrm{~m}, 4 \mathrm{H}), 0.88$ (t, $J=$ 7.2Hz, 2H). ${ }^{13} \mathrm{C} \mathrm{NMR}\left(150.8 \mathrm{MHz}, \mathrm{CDCl}_{3}\right) \delta 146.8,132.5$ (t, $\left.J=3.5 \mathrm{~Hz}\right), 128.8,122.0-$ $116.0\left[\mathrm{~m},-\left(\mathrm{CF}_{2}\right) \mathrm{CF}_{3}\right], 115.6(\mathrm{t}, J=4.4 \mathrm{~Hz}), 113.8-104.5$ [m, $\left.-\left(\mathrm{CF}_{2}\right) \mathrm{CF}_{3}\right], 92.8(\mathrm{t}, J=9.6$ $\mathrm{Hz}), 74.0(\mathrm{t}, J=52.2 \mathrm{~Hz}), 36.1,31.4,30.8,22.5,14.0 .{ }^{19} \mathrm{~F} \mathrm{NMR}\left(564 \mathrm{MHz}, \mathrm{CDCl}_{3}\right) \delta$ $80.2(\mathrm{t}, J=8.5 \mathrm{~Hz}, 3 \mathrm{~F}),-97.9(\mathrm{~m}, 2 \mathrm{~F}),-126.9(\mathrm{t}, J=6.2 \mathrm{~Hz}, 2 \mathrm{~F})$. IR (thin film): $v_{\max } 2928$, 1569, $877 \mathrm{~cm}^{-1}$. MS (EI) m/z: (\%) 340 (M), 283 (100), 164. HRMS (EI) m/z: [M] Calculated for $\mathrm{C}_{16} \mathrm{H}_{15} \mathrm{~F}_{7}$ : 340.1062; Found: 340.1059 .<smiles>FC(F)(F)C(F)(F)C#Cc1ccc(Cl)cc1</smiles>

1-Chloro-4-(perfluoropent-1-yn-1-yl)benzene (5h). The product (40 mg, 66\% yield) as a pale yellow oil was purified with silica gel chromatography (Petroleum ether (100\%). ${ }^{1} \mathrm{H} \mathrm{NMR}\left(600 \mathrm{MHz}, \mathrm{CDCl}_{3}\right) \delta 7.49(\mathrm{~d}, J=9.0 \mathrm{~Hz}, 2 \mathrm{H}), 7.38(\mathrm{~d}, J=8.4 \mathrm{~Hz}, 2 \mathrm{H})$. ${ }^{13} \mathrm{C} \mathrm{NMR}\left(150.8 \mathrm{MHz}, \mathrm{CDCl}_{3}\right) \delta 137.5,133.7$ (t, $\left.J=2.3 \mathrm{~Hz}\right), 129.1,120.5-118.3$ [m, $\left.\left(\mathrm{CF}_{2}\right) \mathrm{CF}_{3}\right], 116.8(\mathrm{t}, J=2.9 \mathrm{~Hz}), 116.7-105.2\left[\mathrm{~m},-\left(\mathrm{CF}_{2}\right) \mathrm{CF}_{3}\right], 90.9(\mathrm{t}, J=6.5 \mathrm{~Hz}), 75.3$ $(\mathrm{t}, J=36.5 \mathrm{~Hz}) .{ }^{19} \mathrm{~F}$ NMR $\left(564 \mathrm{MHz}, \mathrm{CDCl}_{3}\right) \delta-80.1(\mathrm{t}, J=7.9 \mathrm{~Hz}, 3 \mathrm{~F}),-98.5(\mathrm{~m}, 2 \mathrm{~F})$, -126.9 (t, $J=5.6 \mathrm{~Hz}, 2 \mathrm{~F})$. IR (thin film): $v_{\max } 2925,1050,666 \mathrm{~cm}^{-1}$. MS (EI) m/z: (\%) 303 (M), 185 (100), 151. HRMS (EI) m/z: [M] Calculated for $\mathrm{C}_{11} \mathrm{H}_{4} \mathrm{ClF}_{7}$ : 303.9890; Found: 303.9884 .

$\mathrm{H}_{3} \mathrm{C}\left(\mathrm{CH}_{2}\right)_{9}=\mathrm{C}_{3} \mathrm{~F}_{7}$

1,1,1,2,2,3,3-Heptafluoropentadec-4-yne (5l). The product (50 mg, 75\% yield) as a colorless oil was purified with silica gel chromatography (Petroleum ether $(100 \%) .{ }^{1} \mathrm{H}$ 
NMR (600 MHz, $\left.\mathrm{CDCl}_{3}\right) \delta 2.35(\mathrm{dt}, J=12.6 \mathrm{~Hz}, 6.6 \mathrm{~Hz}, 2 \mathrm{H}), 1.59$ (dt, $J=15.0 \mathrm{~Hz}$, $7.2 \mathrm{~Hz}, 2 \mathrm{H}), 1.40(\mathrm{~m}, 2 \mathrm{H}), 1.27(\mathrm{~m}, 13 \mathrm{H}), 0.89(\mathrm{t}, J=6.6 \mathrm{~Hz}, 3 \mathrm{H}) .{ }^{13} \mathrm{C} \mathrm{NMR}(150.8 \mathrm{MHz}$, $\left.\mathrm{CDCl}_{3}\right) \delta 120.5 .0-104.8\left[\mathrm{~m},-\left(\mathrm{CF}_{2}\right) \mathrm{CF}_{3}\right], 95.3(\mathrm{t}, J=6.2 \mathrm{~Hz}), 67.1(\mathrm{t}, J=35.7 \mathrm{~Hz}), 31.8$, 29.4, 29.3, 29.2, 28.8, 28.5, $27.1(\mathrm{t}, J=1.8 \mathrm{~Hz}), 22.6,18.4(\mathrm{t}, J=2.4 \mathrm{~Hz}), 14.0 .{ }^{19} \mathrm{~F}$ NMR (564 MHz, $\left.\mathrm{CDCl}_{3}\right) \delta-80.3(\mathrm{~m}, 3 \mathrm{~F}),-97.4$ (m, 2F), -127.1 (s, 2F). IR (thin film): $v_{\max }$ 2928, 1369, $1020 \mathrm{~cm}^{-1}$. MS (EI) m/z: (\%) 334 (M), 69 (100), 55. HRMS (EI) m/z: $[\mathrm{M}]^{+}$Calculated for $\mathrm{C}_{15} \mathrm{H}_{21} \mathrm{~F}_{7}$ : 334.1531; Found: 334.1531 .

$\mathbf{6 , 6 , 7 , 7 , 8 , 8 , 8 - H e p t a f l u o r o o c t - 4 - y n - 1 - y l ~ a c e t a t e ~ ( 5 n ) . ~ T h e ~ p r o d u c t ~ ( 3 6 ~ m g , ~ 6 1 \% ~ y i e l d ) ~}$ as a pale yellow oil was purified with silica gel chromatography (Petroleum ether / Ethyl acetate $=20: 1) .{ }^{1} \mathrm{H}$ NMR $\left(600 \mathrm{MHz}, \mathrm{CDCl}_{3}\right) \delta 4.13(\mathrm{t}, J=6.6 \mathrm{~Hz}, 2 \mathrm{H}), 2.45(\mathrm{dt}, J=12.6$ $\mathrm{Hz}, 6.0 \mathrm{~Hz}, 2 \mathrm{H}), 2.04$ (s, 3H), 1.91 (dt, $J=13.2 \mathrm{~Hz}, 6.0 \mathrm{~Hz}, 2 \mathrm{H}) .{ }^{13} \mathrm{C} \mathrm{NMR}(150.8 \mathrm{MHz}$, $\left.\mathrm{CDCl}_{3}\right) \delta 170.8,120.2-104.7\left[\mathrm{~m},-\left(\mathrm{CF}_{2}\right) \mathrm{CF}_{3}\right], 93.6(\mathrm{t}, J=6.2 \mathrm{~Hz}), 67.6(\mathrm{t}, J=36.0 \mathrm{~Hz})$, 62.4, $26.4(\mathrm{t}, J=2.0 \mathrm{~Hz}), 20.7,15.4(\mathrm{t}, J=2.4 \mathrm{~Hz}) .{ }^{19} \mathrm{~F} \mathrm{NMR}\left(564 \mathrm{MHz}, \mathrm{CDCl}_{3}\right) \delta$ 80.2 (m, 3F), -97.7 (s, 2F), -120.7 (s, 2F). IR (thin film): $v_{\max } 2925,1742,1147 \mathrm{~cm}^{-1}$. MS (EI) m/z: (\%) 294 (M), 115, 61 (100). HRMS (APCI-LTQ Orbitrap) m/z: [M+H] ${ }^{+}$ Calculated for $\mathrm{C}_{10} \mathrm{H}_{10} \mathrm{~F}_{7} \mathrm{O}_{2}$ : 295.0564; Found: 295.0564 .

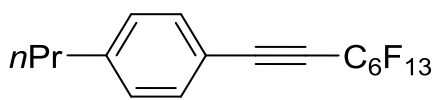

1-(perfluorooct-1-yn-1-yl)-4-propylbenzene (7a). This compound is known. ${ }^{11}$ The product $(79 \mathrm{mg}, 86 \%$ yield) as a pale yellow oil was purified with silica gel chromatography (Petroleum ether (100\%)). ${ }^{1} \mathrm{H}$ NMR $\left(600 \mathrm{MHz}, \mathrm{CDCl}_{3}\right) \delta 7.47(\mathrm{~d}, J=$ $7.8 \mathrm{~Hz}, 2 \mathrm{H}), 7.21(\mathrm{~d}, J=8.4 \mathrm{~Hz}, 2 \mathrm{H}), 2.62(\mathrm{t}, J=7.8 \mathrm{~Hz}, 2 \mathrm{H}), 1.65(\mathrm{~m}, 2 \mathrm{H}), 0.94(\mathrm{t}, J$ $=7.2 \mathrm{~Hz}, 3 \mathrm{H}) .{ }^{19} \mathrm{~F}$ NMR $\left(564 \mathrm{MHz}, \mathrm{CDCl}_{3}\right) \delta-80.9(\mathrm{t}, J=9.6 \mathrm{~Hz}, 3 \mathrm{~F}),-96.8(\mathrm{~s}, 2 \mathrm{~F}),-$ $121.4(\mathrm{~s}, 2 \mathrm{~F}),-122.6$ (s, 2F), -122.9 (s, 2F),-126.2 (s, 2F).

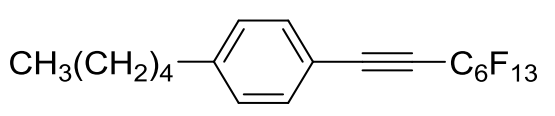

1-pentyl-4-(perfluorooct-1-yn-1-yl)benzene (7b). This compound is known. ${ }^{11}$ The product (88 mg, 90\% yield) as a pale yellow oil was purified with silica gel chromatography (Petroleum ether (100\%)). ${ }^{1} \mathrm{H}$ NMR $\left(600 \mathrm{MHz}, \mathrm{CDCl}_{3}\right) \delta 7.47(\mathrm{~d}, J=$ $7.8 \mathrm{~Hz}, 2 \mathrm{H}), 7.21(\mathrm{~d}, J=7.8 \mathrm{~Hz}, 2 \mathrm{H}), 2.64(\mathrm{t}, J=7.8 \mathrm{~Hz}, 2 \mathrm{H}), 1.61(\mathrm{~m}, 2 \mathrm{H}), 1.32(\mathrm{~m}$, $4 \mathrm{H}), 0.90(\mathrm{t}, J=7.2 \mathrm{~Hz}, 3 \mathrm{H}) .{ }^{19} \mathrm{~F}$ NMR $\left(564 \mathrm{MHz}, \mathrm{CDCl}_{3}\right) \delta-80.9(\mathrm{~d}, J=8.5 \mathrm{~Hz}, 3 \mathrm{~F})$, $-96.8(\mathrm{~s}, 2 \mathrm{~F}),-121.4(\mathrm{~s}, 2 \mathrm{~F}),-122.6(\mathrm{~s}, 2 \mathrm{~F}),-122.9(\mathrm{~s}, 2 \mathrm{~F}),-126.2(\mathrm{~s}, 2 \mathrm{~F})$. 


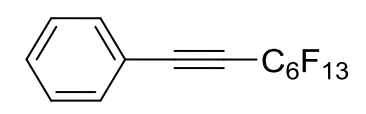

(perfluorooct-1-yn-1-yl)benzene (7c). This compound is known. ${ }^{11}$ The product $(75 \mathrm{mg}$, $89 \%$ yield) as a colorless oil was purified with silica gel chromatography (Petroleum ether (100\%)). ${ }^{1} \mathrm{H}$ NMR (600 MHz, $\left.\mathrm{CDCl}_{3}\right) \delta 7.57$ (d, $\left.J=7.2 \mathrm{~Hz}, 2 \mathrm{H}\right), 7.49$ (t, $J=7.8$ $\mathrm{Hz}, 2 \mathrm{H}), 7.41(\mathrm{t}, J=7.2 \mathrm{~Hz}, 2 \mathrm{H}) .{ }^{19} \mathrm{~F} \mathrm{NMR}\left(564 \mathrm{MHz}, \mathrm{CDCl}_{3}\right) \delta-80.9(\mathrm{t}, J=9.6 \mathrm{~Hz}$, $3 \mathrm{~F}),-97.2(\mathrm{~s}, 2 \mathrm{~F}),-121.4(\mathrm{~s}, 2 \mathrm{~F}),-122.6(\mathrm{~s}, 2 \mathrm{~F}),-122.9(\mathrm{~s}, 2 \mathrm{~F}),-126.2(\mathrm{t}, J=14.1 \mathrm{~Hz}$, 2F).

1-methoxy-4-(perfluorooct-1-yn-1-yl)benzene (7d). This compound is known. ${ }^{11}$ The product (83 mg, 92\% yield) as a pale yellow oil was purified with silica gel chromatography (Petroleum ether (100\%)). ${ }^{1} \mathrm{H}$ NMR $\left(600 \mathrm{MHz}, \mathrm{CDCl}_{3}\right) \delta 7.50(\mathrm{~d}, J=$ $9.0 \mathrm{~Hz}, 2 \mathrm{H}), 6.90(\mathrm{~d}, J=9.0 \mathrm{~Hz}, 2 \mathrm{H}), 3.84(\mathrm{~s}, 3 \mathrm{H}) .{ }^{19} \mathrm{~F} \mathrm{NMR}\left(564 \mathrm{MHz}, \mathrm{CDCl}_{3}\right) \delta-$ $81.0(\mathrm{~d}, J=7.9 \mathrm{~Hz}, 3 \mathrm{~F}),-96.6(\mathrm{~s}, 2 \mathrm{~F}),-121.4(\mathrm{~s}, 2 \mathrm{~F}),-122.6(\mathrm{~s}, 2 \mathrm{~F}),-122.9(\mathrm{~s}, 2 \mathrm{~F}),-$ $126.3(\mathrm{~d}, J=7.9 \mathrm{~Hz}, 2 \mathrm{~F})$.<smiles>[PH3+]C#Cc1ccc(Cl)cc1</smiles>

1-chloro-4-(perfluorooct-1-yn-1-yl)benzene (7h). This compound is known. ${ }^{11}$ The product $(75 \mathrm{mg}, 83 \%$ yield) as a pale yellow oil was purified with silica gel chromatography (Petroleum ether (100\%)). ${ }^{1} \mathrm{H}$ NMR $\left(600 \mathrm{MHz}, \mathrm{CDCl}_{3}\right) \delta 7.50(\mathrm{~d}, J=$ $8.4 \mathrm{~Hz}, 2 \mathrm{H}), 7.40(\mathrm{~d}, J=9.0 \mathrm{~Hz}, 2 \mathrm{H}) .{ }^{19} \mathrm{~F} \mathrm{NMR}\left(564 \mathrm{MHz}, \mathrm{CDCl}_{3}\right) \delta-80.9(\mathrm{t}, J=9.6$ $\mathrm{Hz}, 3 \mathrm{~F}),-97.5$ (s, 2F), -121.4 (s, 2F), -122.5 (s, 2F), -122.9 (s, 2F), -126.2 (d, J= 14.1 $\mathrm{Hz}, 2 \mathrm{~F})$.

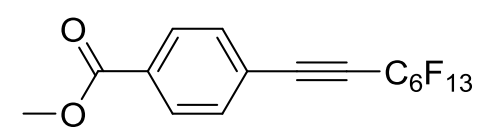

Methyl 4-(perfluorooct-1-yn-1-yl)benzoate (7i). This compound is known. ${ }^{11}$ The product ( $83 \mathrm{mg}, 86 \%$ yield) as a pale yellow oil was purified with silica gel chromatography (Petroleum ether / Ethyl ether $=15: 1) .{ }^{1} \mathrm{H}$ NMR $\left(600 \mathrm{MHz}, \mathrm{CDCl}_{3}\right) \delta$ $8.06(\mathrm{~d}, J=8.4 \mathrm{~Hz}, 2 \mathrm{H}), 7.62(\mathrm{~d}, J=8.4 \mathrm{~Hz}, 2 \mathrm{H}), 3.93$ (s, 3H). ${ }^{19} \mathrm{~F}$ NMR (564 MHz, $\left.\mathrm{CDCl}_{3}\right) \delta-80.9(\mathrm{t}, J=8.5 \mathrm{~Hz}, 3 \mathrm{~F}),-97.8(\mathrm{~s}, 2 \mathrm{~F}),-121.38(\mathrm{~s}, 2 \mathrm{~F}),-122.5(\mathrm{~s}, 2 \mathrm{~F}),-122.9$ $(\mathrm{s}, 2 \mathrm{~F}),-126.2(\mathrm{~s}, 2 \mathrm{~F})$.

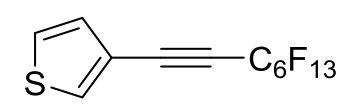

3-(Perfluorooct-1-yn-1-yl)thiophene (7j). The product (71 $\mathrm{mg}, 83 \%$ yield) as a pale yellow oil was purified with silica gel chromatography (Petroleum ether $(100 \%) .{ }^{1} \mathrm{H}$ 
NMR (600 MHz, $\left.\mathrm{CDCl}_{3}\right) \delta 7.73(\mathrm{~d}, J=1.8 \mathrm{~Hz}, 1 \mathrm{H}), 7.34(\mathrm{~m}, 2 \mathrm{H}), 3.95(\mathrm{~d}, J=4.8 \mathrm{~Hz}$, 1H). ${ }^{13} \mathrm{C} \mathrm{NMR}\left(150.8 \mathrm{MHz}, \mathrm{CDCl}_{3}\right) \delta 133.4$ (t, $\left.J=4.2 \mathrm{~Hz}\right), 129.7,126.4,119.0-108.3$ [m, $\left.-\left(\mathrm{CF}_{2}\right) \mathrm{CF}_{3}\right], 117.6(\mathrm{t}, J=4.8 \mathrm{~Hz}), 116.1-104.9$ [m, $\left.-\left(\mathrm{CF}_{2}\right) \mathrm{CF}_{3}\right], 87.7$ (t, $\left.J=9.8 \mathrm{~Hz}\right)$, $74.6(\mathrm{t}, J=54.2 \mathrm{~Hz}) .{ }^{19} \mathrm{~F}$ NMR $\left(564 \mathrm{MHz}, \mathrm{CDCl}_{3}\right) \delta-81.1(\mathrm{t}, J=9.6 \mathrm{~Hz}, 3 \mathrm{~F}),-97.2$ (m,2F), -121.5 (s, 2F), -122.6(m, 2F), -123.0(m, 2F), -126.4(m, 2F). MS (EI) m/z: (\%) 362 (M), 333, 164 (100). IR (thin film): $v_{\max }$ 2975, 1202, $1005 \mathrm{~cm}^{-1}$. MS (EI) m/z: (\%) 425 (M), 157 (100), 69. HRMS (APCI-LTQ Orbitrap) m/z: [M+H] ${ }^{+}$Calculated for $\mathrm{C}_{12} \mathrm{H}_{4} \mathrm{~F}_{13} \mathrm{~S}$ : 426.9821 ; Found: 426.9822.<smiles>[R5]C(F)(F)C#Cc1cccnc1</smiles>

3-(Perfluorohex-1-yn-1-yl)pyridine (7k). The product (61 mg, 73\% yield) as a pale yellow oil was purified with silica gel chromatography (Petroleum ether $(100 \%) .{ }^{1} \mathrm{H}$ NMR $\left(600 \mathrm{MHz}, \mathrm{CDCl}_{3}\right) \delta 8.77(\mathrm{~s}, 1 \mathrm{H}), 8.68(\mathrm{~d}, J=4.8 \mathrm{~Hz}, 1 \mathrm{H}), 7.84(\mathrm{~d}, J=7.8 \mathrm{~Hz}$, 1H), $7.33(\mathrm{~m}, 1 \mathrm{H}) .{ }^{13} \mathrm{C} \mathrm{NMR}\left(150.8 \mathrm{MHz}, \mathrm{CDCl}_{3}\right) \delta 152.8(\mathrm{t}, J=2.4 \mathrm{~Hz}), 151.2,139.4$, 132.1, 120.2-115.9 [m, - $\left.\left(\mathrm{CF}_{2}\right) \mathrm{CF}_{3}\right], 115.8(\mathrm{t}, J=2.9 \mathrm{~Hz}), 114.4-105.5$ [m, $\left.-\left(\mathrm{CF}_{2}\right) \mathrm{CF}_{3}\right]$, $88.7(\mathrm{t}, J=6.3 \mathrm{~Hz}), 77.6(\mathrm{t}, J=36.3 \mathrm{~Hz}) .{ }^{19} \mathrm{~F} \mathrm{NMR}\left(564 \mathrm{MHz}, \mathrm{CDCl}_{3}\right) \delta-85.9(\mathrm{~m}, 3 \mathrm{~F})$, -103.1 (s,2F), -128.2 (s, 2F), -130.3(s, 2F). IR (thin film): $v_{\max } 2925,1239,1147 \mathrm{~cm}^{-1}$. MS (EI) m/z: (\%) 421 (M), 152 (100), 99. HRMS (APCI-LTQ Orbitrap) m/z: [M+H] ${ }^{+}$ Calculated for $\mathrm{C}_{13} \mathrm{H}_{5} \mathrm{~F}_{13} \mathrm{~N}$ : 422.0209 ; Found: 422.0208 .

$\mathrm{H}_{3} \mathrm{C}\left(\mathrm{CH}_{2}\right)_{9}=\mathrm{C}_{6} \mathrm{~F}_{13}$

1,1,1,2,2,3,3,4,4,5,5,6,6-Tridecafluorooctadec-7-yne (7l). The product ( $85 \mathrm{mg}, 88 \%$ yield) as a colorless oil was purified with silica gel chromatography (Petroleum ether (100\%). ${ }^{1} \mathrm{H}$ NMR (600 MHz, $\left.\mathrm{CDCl}_{3}\right) \delta 2.33$ (t, 2H), 1.57 (dt, $\left.J=14.4 \mathrm{~Hz}, 7.2 \mathrm{~Hz}, 2 \mathrm{H}\right)$, $2.13(\mathrm{~m}, 2 \mathrm{H}), 1.26(\mathrm{~m}, 13 \mathrm{H}), 0.87(\mathrm{t}, J=6.6 \mathrm{~Hz}, 3 \mathrm{H}) .{ }^{13} \mathrm{C} \mathrm{NMR}\left(150.8 \mathrm{MHz}, \mathrm{CDCl}_{3}\right) \delta$ 120.0-105.2 [m, - $\left.\left(\mathrm{CF}_{2}\right) \mathrm{CF}_{3}\right], 95.4(\mathrm{t}, J=6.0 \mathrm{~Hz}), 67.3(\mathrm{t}, J=35.6 \mathrm{~Hz}), 31.8,29.4,29.3$, 29.2, 28.9, 28.5, $27.1(\mathrm{t}, J=1.5 \mathrm{~Hz}), 22.6,18.4(\mathrm{t}, J=6.0 \mathrm{~Hz}), 14.0 .{ }^{19} \mathrm{~F}$ NMR $(564$ $\left.\mathrm{MHz} \mathrm{CDCl}_{3}\right) \delta-81.0(\mathrm{t}, J=11.1 \mathrm{~Hz}, 3 \mathrm{~F}),-96.3(\mathrm{~s}, 2 \mathrm{~F}),-121.4(\mathrm{~s}, 2 \mathrm{~F}),-122.7(\mathrm{~s}, 2 \mathrm{~F}),-$ $123.0(\mathrm{~s}, 2 \mathrm{~F}),-126.3$ (s, 2F). IR (thin film): $v_{\max } 2931,1242,1145 \mathrm{~cm}^{-1} . \mathrm{MS}(\mathrm{EI}) \mathrm{m} / \mathrm{z}$ : (\%) 484 (M), 399, 69 (100). HRMS (EI) m/z: [M] Calculated for $\mathrm{C}_{18} \mathrm{H}_{21} \mathrm{~F}_{13}$ : 484.1436; Found: 484.1433.

$\mathrm{TBSO}\left(\mathrm{CH}_{2}\right)_{3}=\mathrm{C}_{6} \mathrm{~F}_{13}$

tert-Butyldimethyl((6,6,7,7,8,8,9,9,10,10,11,11,11-tridecafluoroundec-4-yn-1yl)oxy)silane (7m). The product (72 $\mathrm{mg}, 70 \%$ yield) as a colorless oil was purified with 
silica gel chromatography (Petroleum ether (100\%). ${ }^{1} \mathrm{H}$ NMR $\left(600 \mathrm{MHz}, \mathrm{CDCl}_{3}\right) \delta 3.67$ (t, $J=6.0 \mathrm{~Hz}, 2 \mathrm{H}), 2.45(\mathrm{dt}, J=12.6 \mathrm{~Hz}, 6.6 \mathrm{~Hz}, 2 \mathrm{H}), 1.77(\mathrm{dt}, J=12.6 \mathrm{~Hz}, 7.2 \mathrm{~Hz}, 2 \mathrm{H})$, $0.88(\mathrm{~s}, 9 \mathrm{H}), 0.04(\mathrm{~s}, 6 \mathrm{H}) .{ }^{13} \mathrm{C} \mathrm{NMR}\left(150.8 \mathrm{MHz}, \mathrm{CDCl}_{3}\right) \delta 121.3-104.3\left[\mathrm{~m},-\left(\mathrm{CF}_{2}\right) \mathrm{CF}_{3}\right]$, $95.0(\mathrm{t}, J=5.9 \mathrm{~Hz}), 67.3(\mathrm{t}, J=35.7 \mathrm{~Hz}), 60.7,30.2,29.7,25.7,18.2,14.9,5.7 .{ }^{19} \mathrm{~F}$ NMR (564 MHz, $\left.\mathrm{CDCl}_{3}\right) \delta-80.9(\mathrm{t}, J=9.0 \mathrm{~Hz}, 3 \mathrm{~F}),-96.3(\mathrm{~s}, 2 \mathrm{~F}),-121.4(\mathrm{~s}, 2 \mathrm{~F}),-122.7$ (s,2F), -123.0 (s,2F), -126.2 (s, 2F). IR (thin film): $v_{\max } 2258,1364,1147 \mathrm{~cm}^{-1}$. MS (EI) m/z: (\%) 526 (M), 146, 77 (100). HRMS (EI) m/z: $[\mathrm{M}+\mathrm{H}]^{+}$Calculated for $\mathrm{C}_{17} \mathrm{H}_{22} \mathrm{~F}_{13} \mathrm{OSi}$ : 517.1227; Found: 517.1227.

$(\mathrm{EtO})_{2} \mathrm{OPO}\left(\mathrm{CH}_{2}\right)_{3}=\mathrm{C}_{6} \mathrm{~F}_{13}$

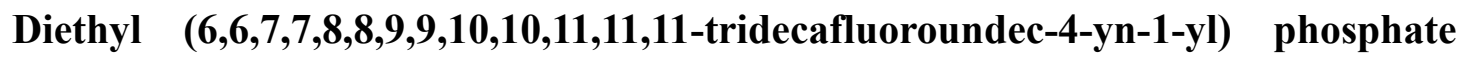
(7q). The product ( $81 \mathrm{mg}, 75 \%$ yield) as a pale yellow oil was purified with silica gel chromatography (dichloromethane / methyl alcohol $=20: 1)$. ${ }^{1} \mathrm{H}$ NMR $(600 \mathrm{MHz}$, $\left.\mathrm{CDCl}_{3}\right) \delta 4.09(\mathrm{~m}, 6 \mathrm{H}), 2.50(\mathrm{dt}, J=12.0 \mathrm{~Hz}, 6.0 \mathrm{~Hz}, 2 \mathrm{H}), 1.93(\mathrm{dt}, J=7.2 \mathrm{~Hz}, 6.0 \mathrm{~Hz})$, $1.30(\mathrm{t}, J=6.6 \mathrm{~Hz}, 3 \mathrm{H}) .{ }^{13} \mathrm{C} \mathrm{NMR}\left(150.8 \mathrm{MHz}, \mathrm{CDCl}_{3}\right) \delta 119.0-106.4\left[\mathrm{~m},-\left(\mathrm{CF}_{2}\right) \mathrm{CF}_{3}\right]$, $93.6(\mathrm{t}, J=8.9 \mathrm{~Hz}), 67.9(\mathrm{t}, J=53.8 \mathrm{~Hz}), 65.3,63.9,28.0,16.1,14.9(\mathrm{t}, J=3.6 \mathrm{~Hz}) .{ }^{19} \mathrm{~F}$ NMR (564 MHz, $\left.\mathrm{CDCl}_{3}\right) \delta-81.1$ (m, 3F), -96.7 (m, 2F), -121.5 (s, 2F), -122.7 (s, 2F), $-123.0(\mathrm{~s}, 2 \mathrm{~F}),-126.3(\mathrm{~m}, 2 \mathrm{~F})$. IR (thin film): $v_{\max } 225,1258,1040 \mathrm{~cm}^{-1}$. MS (EI) m/z: (\%) 538 (M), 127, 99 (100). HRMS (APCI-LTQ Orbitrap) m/z: [M+H] $]^{+}$Calculated for $\mathrm{C}_{15} \mathrm{H}_{17} \mathrm{~F}_{13} \mathrm{O}_{4} \mathrm{P}: 539.0651$; Found: 539.0652 .

$\mathrm{TsO}\left(\mathrm{CH}_{2}\right)_{3}=\mathrm{C}_{6} \mathrm{~F}_{13}$

\section{6,6,7,7,8,8,9,9,10,10,11,11,11-Tridecafluoroundec-4-yn-1-yl4-methylbenzene-}

sulfonate (7r). The product ( $96 \mathrm{mg}, 87 \%$ yield) as a pale yellow oil was purified with silica gel chromatography (Petroleum ether / Ethyl acetate $=10: 1) .{ }^{1} \mathrm{H}$ NMR $(600 \mathrm{MHz}$, $\left.\mathrm{CDCl}_{3}\right) \delta 7.79(\mathrm{~d}, J=7.8 \mathrm{~Hz}, 2 \mathrm{H}), 7.35(\mathrm{~d}, J=8.4 \mathrm{~Hz}, 2 \mathrm{H}), 4.10(\mathrm{t}, J=6.0 \mathrm{~Hz}, 2 \mathrm{H})$, $2.46(\mathrm{~m}, 5 \mathrm{H}), 1.94(\mathrm{dt}, J=13.2 \mathrm{~Hz}, 6.0 \mathrm{~Hz}, 2 \mathrm{H}) .{ }^{13} \mathrm{C} \mathrm{NMR}\left(150.8 \mathrm{MHz}, \mathrm{CDCl}_{3}\right) \delta 145.1$, 132.6, 129.9, 127.8, 119.7-105.1 [m, - $\left.\left(\mathrm{CF}_{2}\right) \mathrm{CF}_{3}\right], 92.7(\mathrm{t}, J=6.0 \mathrm{~Hz}), 68.2(\mathrm{t}, J=36.0$ $\mathrm{Hz}), 67.8,26.7,21.4,14.8(\mathrm{t}, J=2.4 \mathrm{~Hz}) .{ }^{19} \mathrm{~F} \mathrm{NMR}\left(564 \mathrm{MHz}, \mathrm{CDCl}_{3}\right) \delta-80.9(\mathrm{t}, J=$ 10.2Hz, 3F), -96.9 (s, 2F), -121.4 (s, 2F),-122.5 (s, 2F), -122.9 (s, 2F), -126.2 (m, 2F). IR (thin film): $v_{\max } 2258,1364,1147 \mathrm{~cm}^{-1}$. MS (EI) m/z: (\%) 556 (M), 155, 91 (100). HRMS (APCI-LTQ Orbitrap) m/z: $[\mathrm{M}+\mathrm{H}]^{+}$Calculated for $\mathrm{C}_{18} \mathrm{H}_{14} \mathrm{~F}_{13} \mathrm{O}_{3} \mathrm{~S}: 557.0451$; Found: 557.0451. 
Method A:

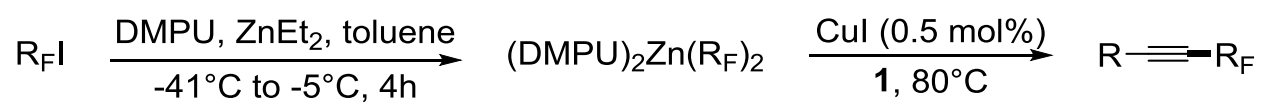

To a septum capped $25 \mathrm{~mL}$ of sealed tube were added DMPU (77 mg, $0.6 \mathrm{mmol}$ ), $\mathrm{C}_{4} \mathrm{~F}_{9} \mathrm{I}$ (221 mg, $0.64 \mathrm{mmol})$ under $\mathrm{N}_{2}$, followed by toluene $(2.0 \mathrm{~mL})$ with stirring. The reaction mixture is cooled to $-41^{\circ} \mathrm{C}$ and $\mathrm{ZnEt}_{2}(0.3 \mathrm{mmol}, 1 \mathrm{M}$ in hexane $)$ is added dropwise to the solution. After stirring at $-5^{\circ} \mathrm{C}$ for $4 \mathrm{~h}$, the sealed tube were added $\mathrm{CuI}(0.5 \mathrm{~mol} \%)$ and $1 \mathrm{a}(0.2 \mathrm{mmol})$ under $\mathrm{N}_{2}$ at room temperature and then heated to $80{ }^{\circ} \mathrm{C}$ (oil bath). After stirring for $6 \mathrm{~h}$, the reaction mixture was cooled to room temperature. The reaction mixture was diluted with ethyl acetate, washed with brine, dried over $\mathrm{Na}_{2} \mathrm{SO}_{4}$, filtered and concentrated. The residue was purified with silica gel chromatography to provide pure product.

Method B:

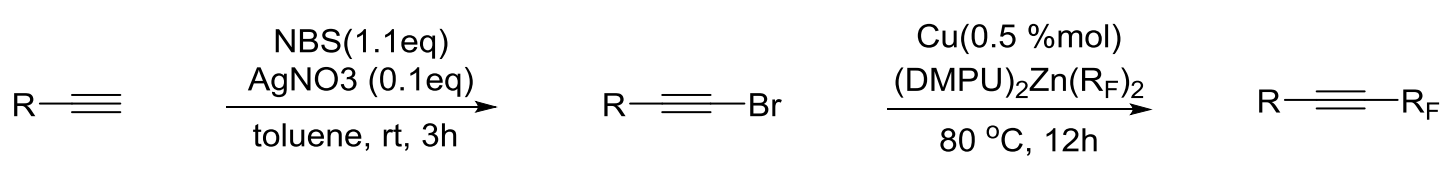

$\mathrm{AgNO}_{3}(3.4 \mathrm{mg}, 0.02 \mathrm{mmol})$ was added to a solution of alkynyl derivatives $(0.2 \mathrm{mmol})$ in toluene $(4 \mathrm{~mL})$. Then NBS (39 $\mathrm{mg}, 0.22 \mathrm{mmol})$ was added in portion. The mixture was stirred for $3 \mathrm{~h}$ at room temperature, then $\mathrm{CuI}(0.5 \mathrm{~mol} \%)$ and (DMPU $)_{2} \mathrm{Zn}\left(\mathrm{R}_{\mathrm{F}}\right)_{2}(1.0$ equiv) were added under $\mathrm{N}_{2}$, and heated to $80^{\circ} \mathrm{C}$ (oil bath). After stirring for $12 \mathrm{~h}$, the reaction mixture was cooled to room temperature. The reaction mixture was diluted with ethyl acetate, washed with brine, dried over $\mathrm{Na}_{2} \mathrm{SO}_{4}$, filtered and concentrated. The residue was purified with silica gel chromatography to provide pure product.

\section{Gram-scale synthesis of copper-catalyzed perfluoroalkylation of alkynyl bromides}

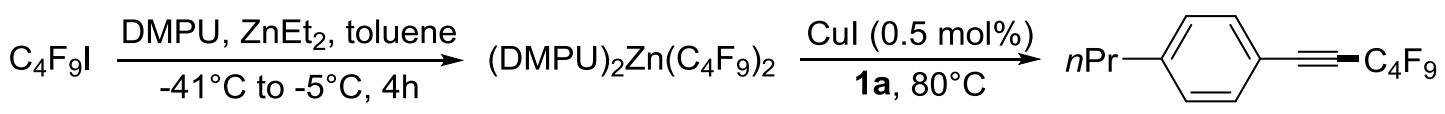

$4.42 \mathrm{~g}, 12.8 \mathrm{mmol}$

$6 a, 1.28 \mathrm{~g}(89 \%)$

$\mathrm{C}_{4} \mathrm{~F}_{9} \mathrm{I}(4.42 \mathrm{~g}, 12.8 \mathrm{mmol})$ and DMPU (1.54 g, $\left.12 \mathrm{mmol}\right)$ were added to by toluene (10 $\mathrm{mL}$ ) under $\mathrm{N}_{2}$. $\mathrm{ZnEt}_{2}$ (6 mmol, $1 \mathrm{M}$ in hexane) was added dropwise to the solution at $41^{\circ} \mathrm{C}$. After stirring at $-5^{\circ} \mathrm{C}$ for $4 \mathrm{~h}$, the sealed tube were added $\mathrm{CuI}(0.5 \mathrm{~mol} \%), \mathbf{1 a}(4.0$ mmol) under $\mathrm{N}_{2}$ at room temperature and heated to $100{ }^{\circ} \mathrm{C}$ (oil bath). After stirring for $6 \mathrm{~h}$, the reaction mixture was cooled to room temperature. The reaction mixture was 
diluted with ethyl acetate, washed with brine, dried over $\mathrm{Na}_{2} \mathrm{SO}_{4}$, filtered and concentrated. The residue was purified with silica gel chromatography (Petroleum ether $(100 \%))$ to provide pure product $(1.28 \mathrm{~g}, 89 \%)$.

\section{The NMR spectroscopic monitoring of copper species using (DMPU) ${ }_{2} \mathrm{Zn}\left(\mathrm{C}_{4} \mathrm{~F}_{9}\right)_{2}$} and CuI.

$\mathrm{CuI}(38.2 \mathrm{mg}, 0.2 \mathrm{mmol})$ and (DMPU $)_{2} \mathrm{Zn}\left(\mathrm{nC}_{4} \mathrm{~F}_{9}\right)_{2}(152 \mathrm{mg}, 0.2 \mathrm{mmol})$ were added to a $25 \mathrm{ml}$ Schlenk tube equipped with a magnetic stir bar. Then, toluene $(2 \mathrm{ml}$, with $1 \%$ volume of fluorobenzene as internal standard) was added under $\mathrm{N}_{2}$. The mixture was stirred for $1 \mathrm{~h}$ at room temperature under. The reaction was monitored by ${ }^{19} \mathrm{~F}$ NMR analysis and $\mathrm{CDCl}_{3}$ was sealed capillary filled with $\mathrm{CDCl}_{3}$ for signal lock. At room temperature, transmetallation reaction was not observed. The reaction mixture was heated to $80{ }^{\circ} \mathrm{C}$, then monitored $0.5 \mathrm{~h}, 1.5 \mathrm{~h}$ and $4 \mathrm{~h}$ after heating. A group of peaks [$81.53(3 \mathrm{~F}),-123.13(2 \mathrm{~F}),-130.52(2 \mathrm{~F}),-137.54(2 \mathrm{~F})]$ can be observed clearly and increased with the reaction time. ${ }^{12}$

$$
\mathrm{Cul}+(\mathrm{DMPU})_{2} \mathrm{Zn}\left(\mathrm{C}_{4} \mathrm{~F}_{9}\right)_{2} \underset{80^{\circ} \mathrm{C}}{\stackrel{\text { toluene }}{\longrightarrow}}\left[\left(\mathrm{C}_{4} \mathrm{~F}_{9}\right)_{n} \mathrm{Cu}\right]^{-(n-2)}
$$

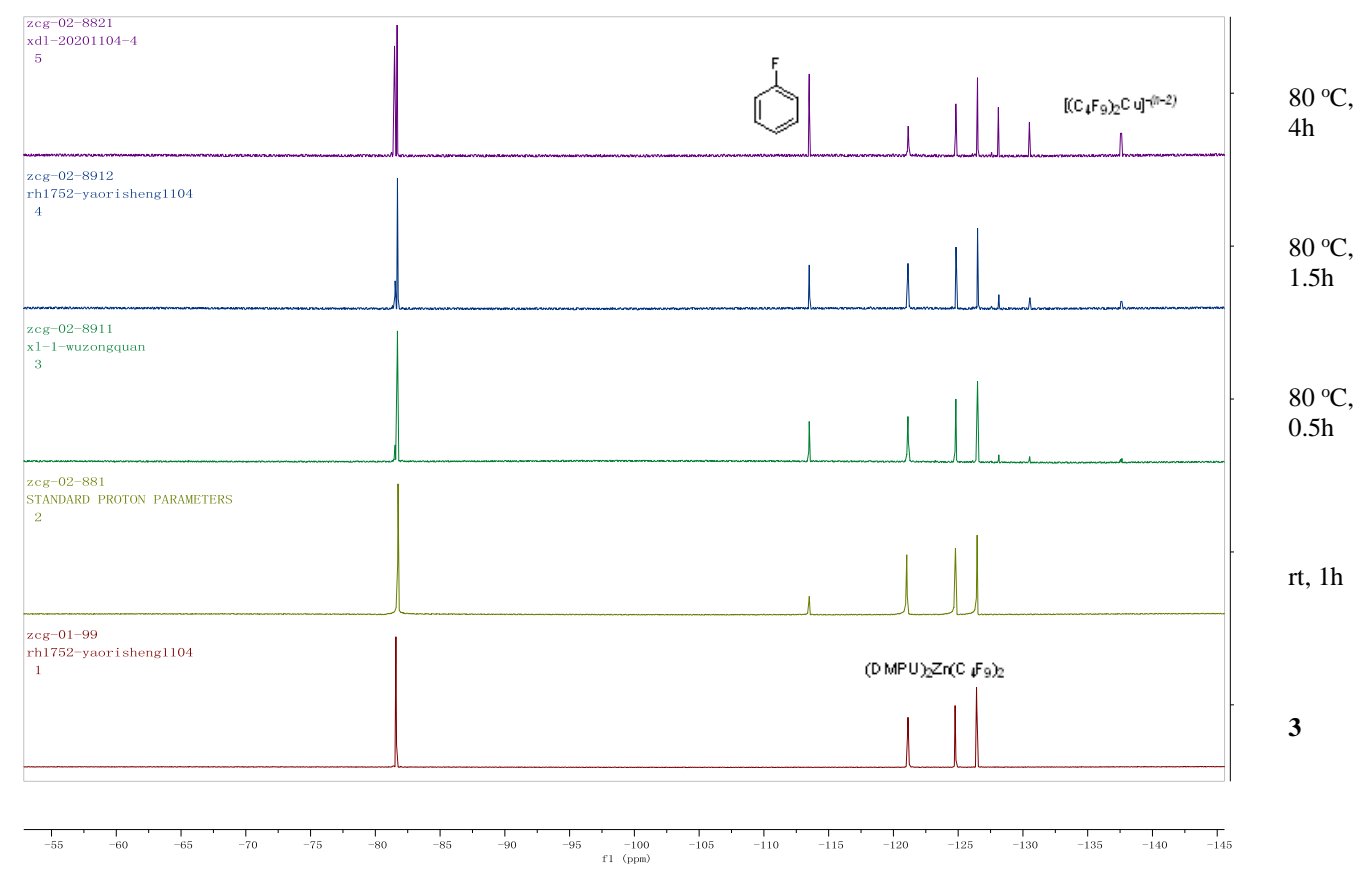




\section{Reference:}

(1) Yang, Y.; Meng, X.; Zhu, B.; Jia, Y.; Cao, X.; Huang, S., A Micellar Catalysis Strategy for Amidation of Alkynyl Bromides: Synthesis of Ynamides in Water. Eur J Org Chem 2019, 2019, 1166-1169.

(2) Hogendorf, W. F. J.; Verhagen, C. P.; Malta, E.; Goosen, N.; Overkleeft, H. S.; Filippov, D. V.; Van der Marel, G. A., The synthesis of a menthol derivative of 2-aminopurine as a fluorescent DNA lesion. Tetrahedron 2009, 65, 10430-10435.

(3) Rajbongshi, K. K.; Hazarika, D.; Phukan, P., TsNBr2 mediated oxidative functionalization of alkynes. Tetrahedron 2016, 72, 4151-4158.

(4) Zhao, Y.; Jin, J.; Chan, P. W. H., Gold Catalyzed Photoredox C1 - Alkynylation of N Alkyl - 1,2,3,4 - tetrahydroisoquinolines by 1 - Bromoalkynes with UVA LED Light. Adv. Synth. Catal. 2018, 361, 1313-1321.

(5) Yao, M. L.; Reddy, M. S.; Yong, L.; Walfish, I.; Blevins, D. W.; Kabalka, G. W., Chemoselective bromodeboronation of organotrifluoroborates using tetrabutylammonium tribromide: application in (Z)-dibromoalkene syntheses. Org. Lett. 2010, 12, 700-703.

(6) Okutani, M.; Mori, Y., Conversion of bromoalkenes into alkynes by wet tetra-nbutylammonium fluoride. J. Org. Chem. 2009, 74, 442-444.

(7) Huang, X.; Chen, H.; Huang, Z.; Xu, Y.; Li, F.; Ma, X.; Chen, Y., Visible Light-Induced Difunctionalization of Alkynes: The Synthesis of Thiazoles and 1,1-Dibromo-1-en-3-ynes. $J$. Org. Chem. 2019, 84, 15283-15293.

(8) Bourkhis, M.; Gaspard, H.; Rulliere, P.; de Almeida, D. K. C.; Listunov, D.; Joly, E.; Abderrahim, R.; de Mattos, M. C.; de Oliveira, M. C. F.; Maraval, V.; Chauvin, R.; Genisson, Y., Skeletal Optimization of Cytotoxic Lipidic Dialkynylcarbinols. ChemMedChem 2018, 13, 1124-1130.

(9) Chinta, B. S.; Baire, B., First Synthesis of the [5-5-6-6] Tetracyclic Framework of Spiropreussione B. Eur. J. Org. Chem. 2017, 2017, 3457-3460.

(10) Goldup, S. M.; Leigh, D. A.; Long, T.; McGonigal, P. R.; Symes, M. D.; Wu, J., Active metal template synthesis of [2]catenanes. J. Am. Chem. Soc. 2009, 131, 15924-15929.

(11) Li, J.; Liu, L.; Zheng, K.; Zheng, C.; Xiao, H.; Fan, S., Silver-Mediated Perfluoroalkylation of Terminal Alkynes with Perfluoroalkyl Iodides. J. Org. Chem. 2020, 85, 8723-8731.

(12) Aikawa, K.; Nakamura, Y.; Yokota, Y.; Toya, W.; Mikami, K., Stable but reactive perfluoroalkylzinc reagents: application in ligand-free copper-catalyzed perfluoroalkylation of aryl iodides. Chem. Eur. J. 2015, 21, 96-100. 
Copies of ${ }^{1} \mathrm{H}$ NMR, ${ }^{19} \mathrm{~F}$ NMR and ${ }^{13} \mathrm{C}$ NMR spectra

\section{3-O-Propagylestrone}

${ }^{1} \mathbf{H}$ NMR $\left(600 \mathrm{MHz}, \mathrm{CDCl}_{3}\right)$

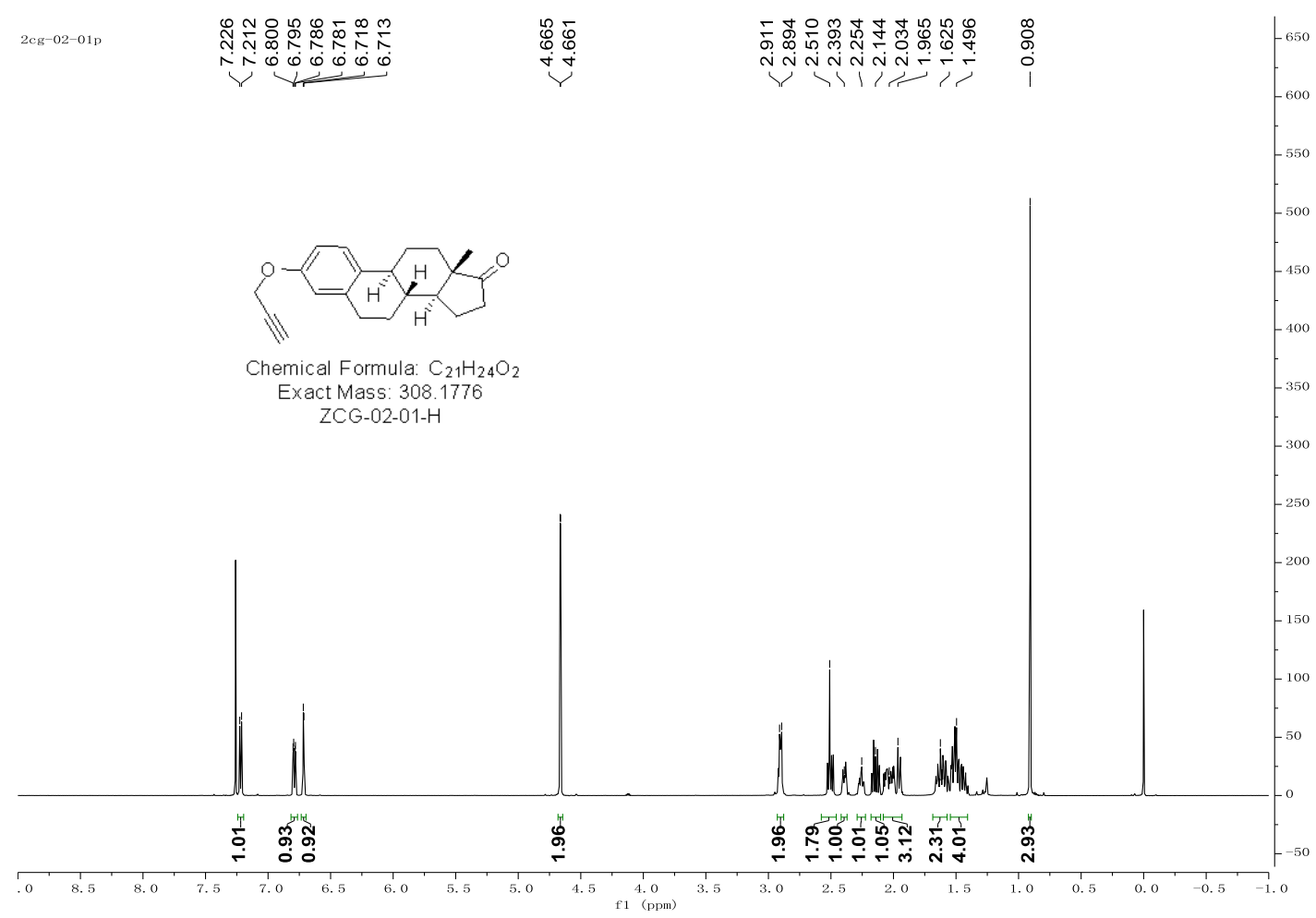

L-Menthyl propargyl ether

${ }^{1} \mathbf{H}$ NMR $\left(600 \mathrm{MHz}, \mathrm{CDCl}_{3}\right)$

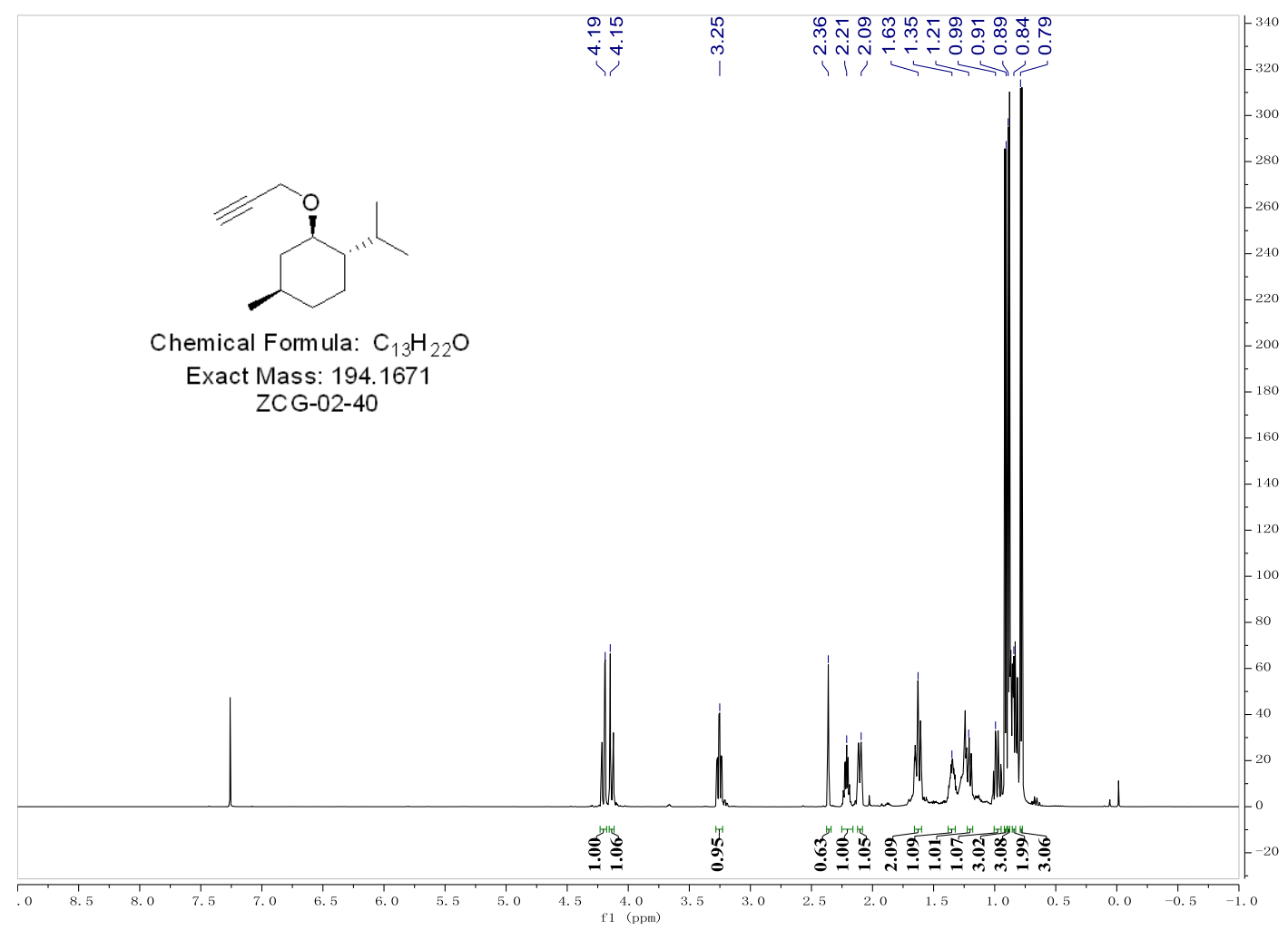


(E)- 1-(Bromoethynyl)-4-propylbenzene (1a)

${ }^{1} \mathbf{H}$ NMR (600 MHz, $\left.\mathrm{CDCl}_{3}\right)$

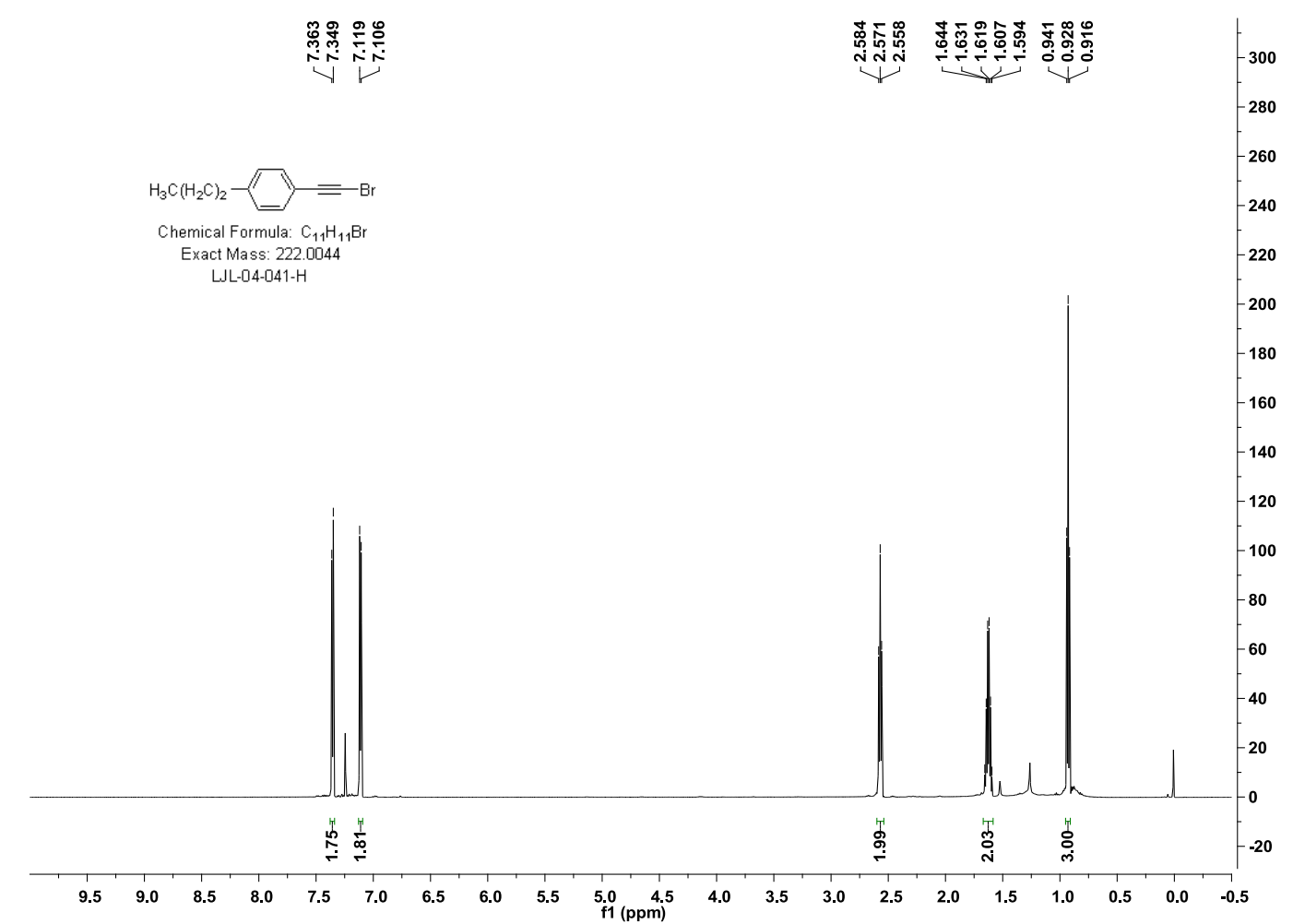

(Bromoethynyl)-4-pentylbenzene (1b)

${ }^{1} \mathbf{H}$ NMR (600 MHz, $\left.\mathrm{CDCl}_{3}\right)$

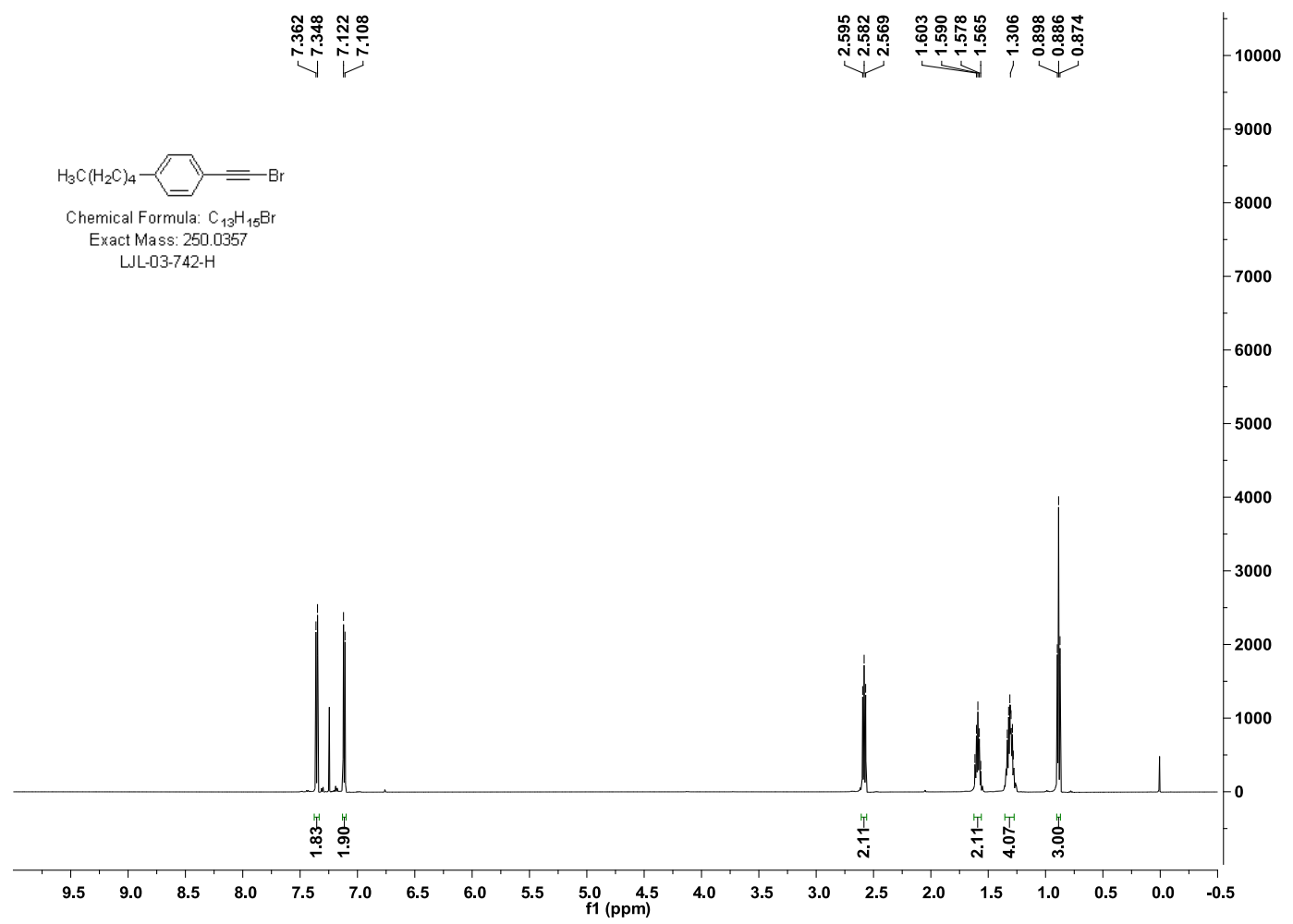


(Bromoethynyl)benzene (1c)

${ }^{1} \mathbf{H}$ NMR (600 MHz, $\left.\mathrm{CDCl}_{3}\right)$

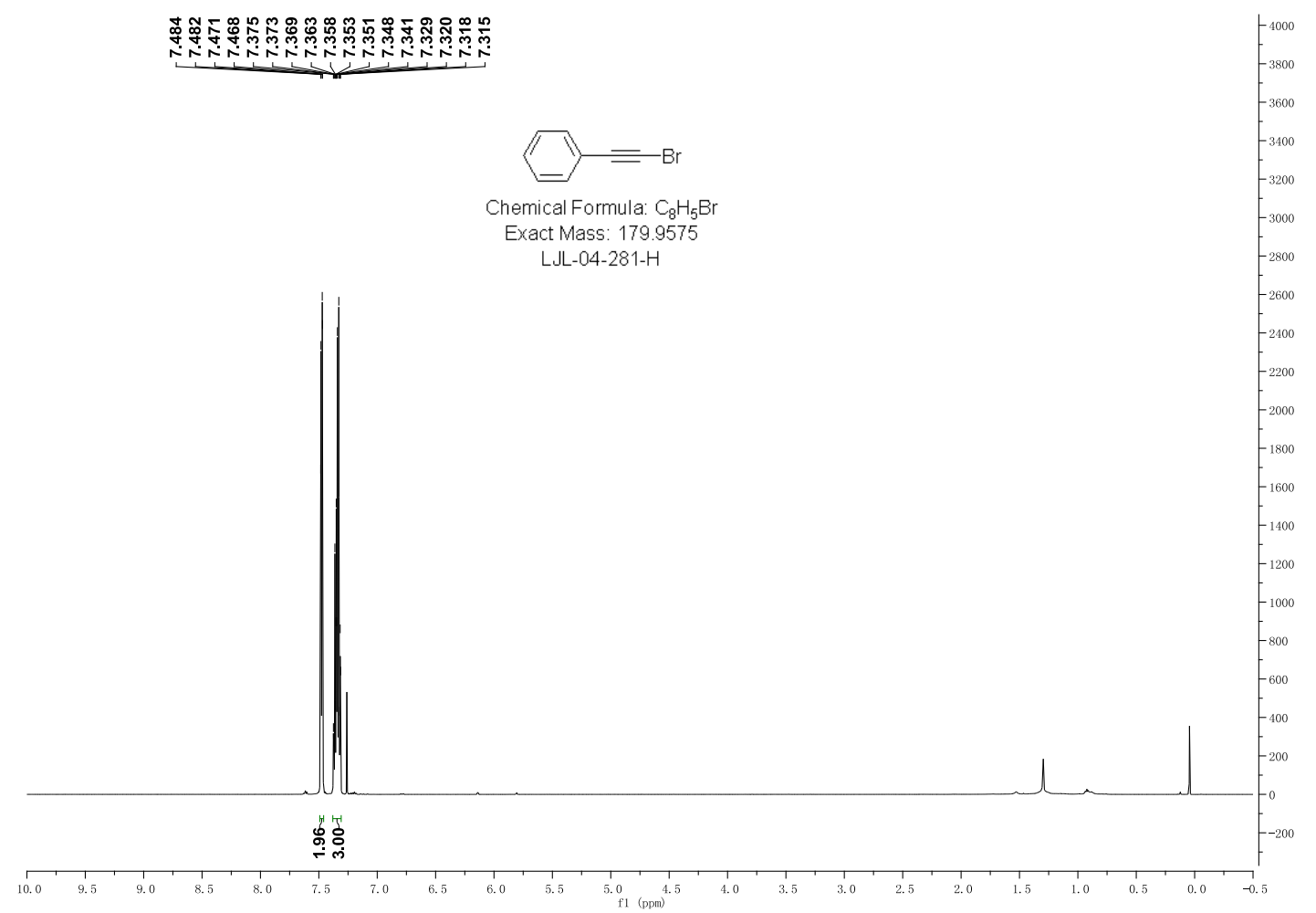

1-(Bromoethynyl)-4-methoxybenzene (1d)

${ }^{1} \mathbf{H}$ NMR (600 MHz, $\left.\mathrm{CDCl}_{3}\right)$

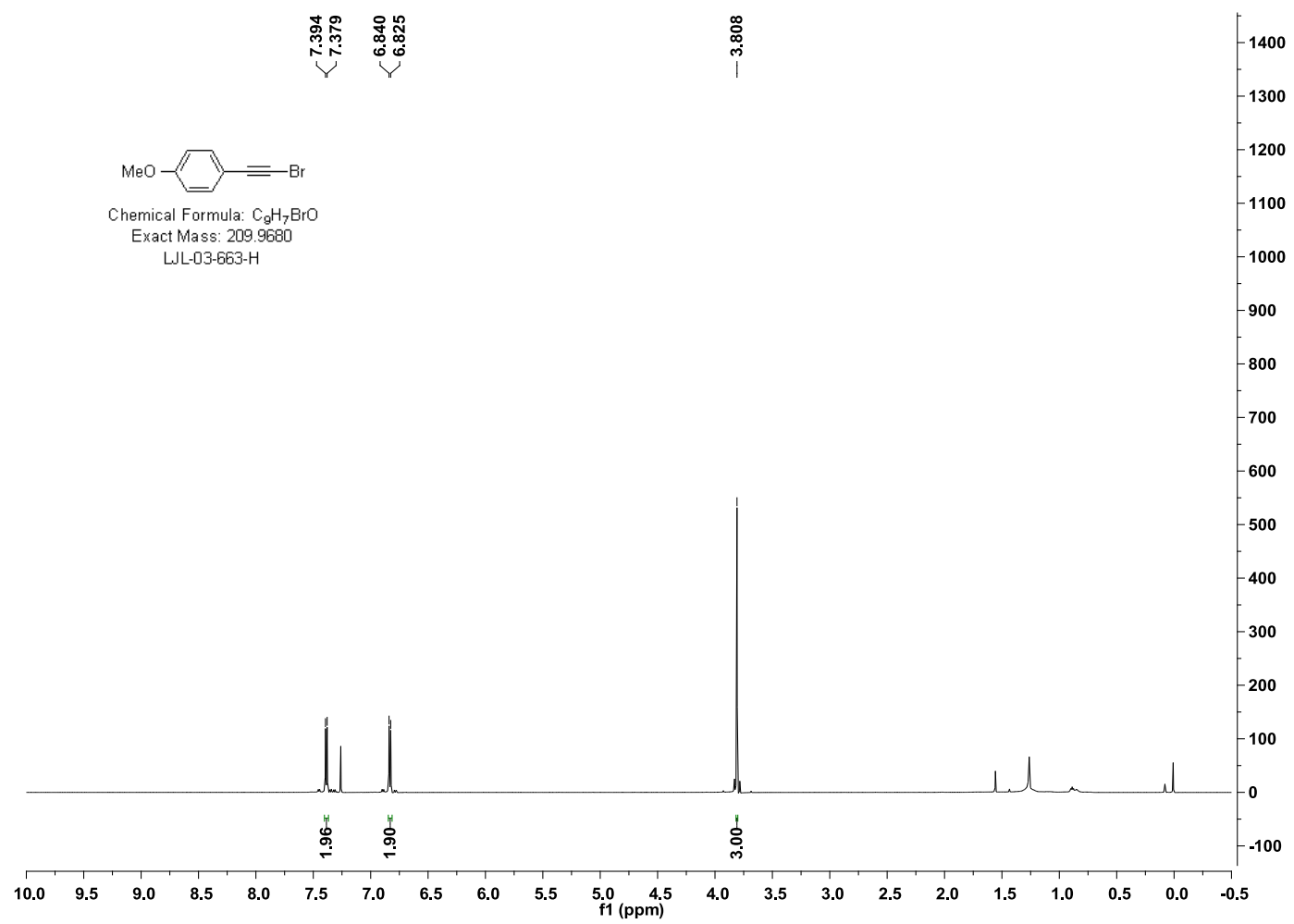


1-(Bromoethynyl)-3,5-dimethoxybenzene (1e)

${ }^{1} \mathbf{H}$ NMR (600 MHz, $\left.\mathrm{CDCl}_{3}\right)$

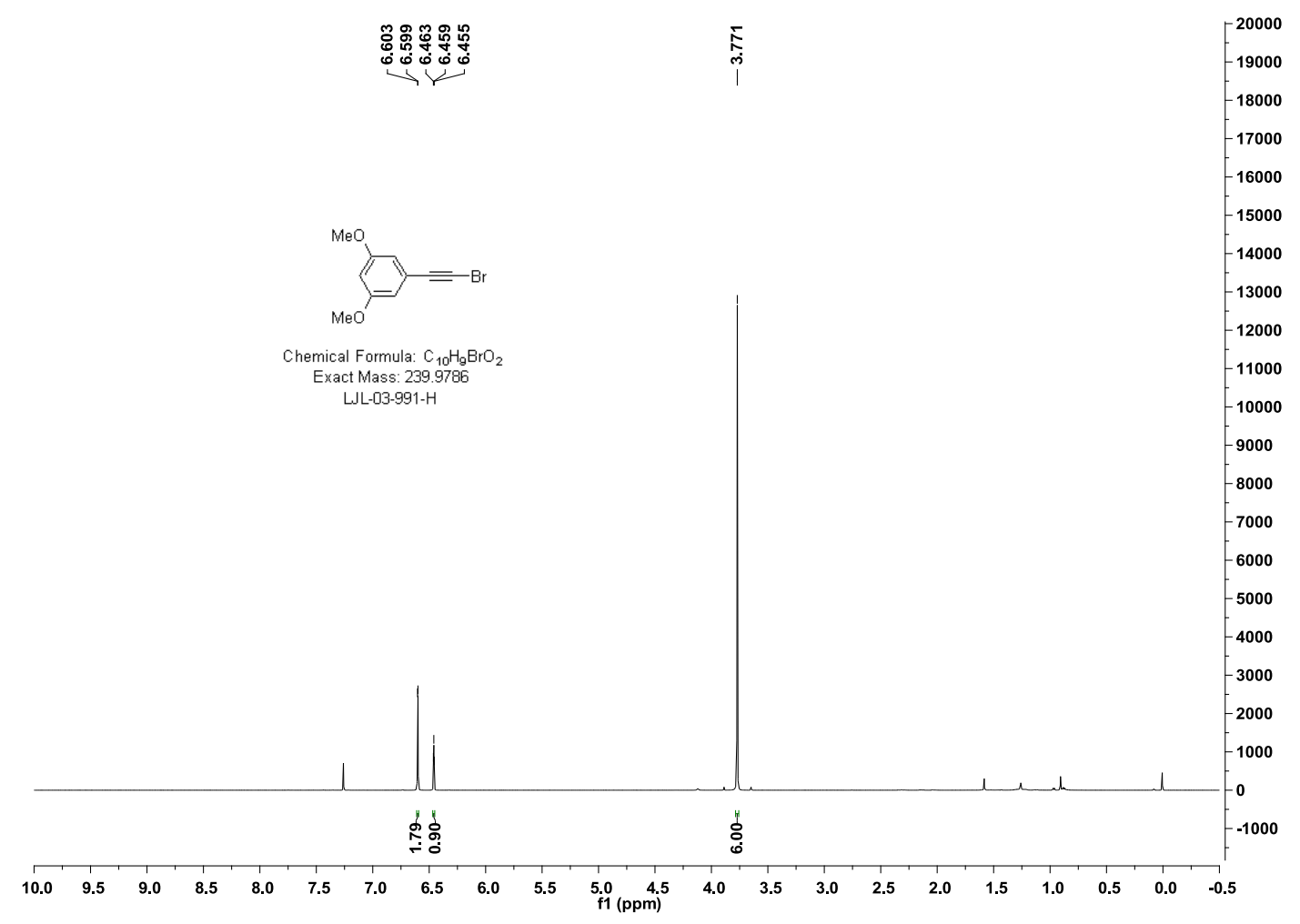

4-(Bromoethynyl)-1,1'-biphenyl (1f)

${ }^{1} \mathbf{H}$ NMR (600 MHz, $\left.\mathrm{CDCl}_{3}\right)$

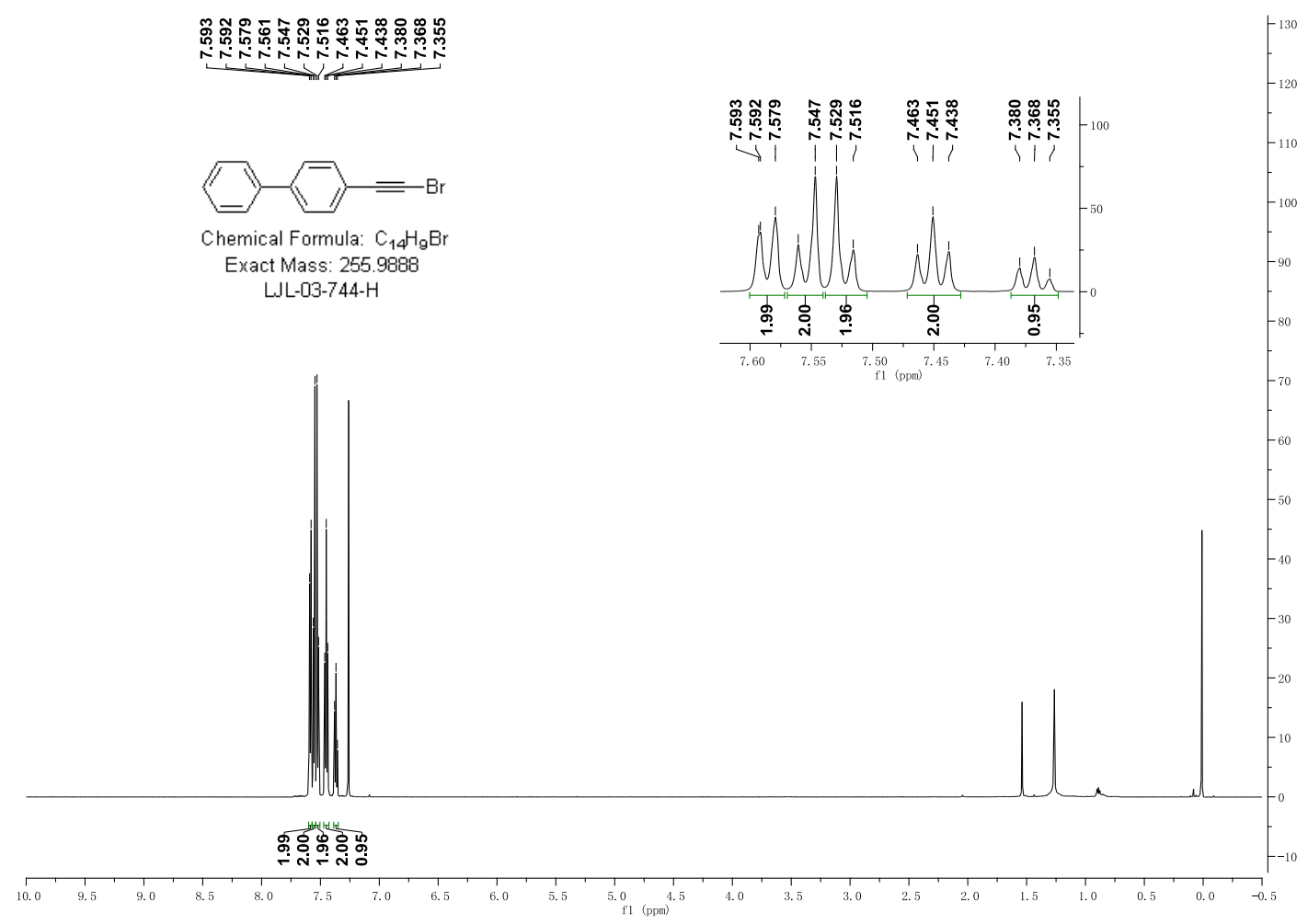


2-(Bromoethynyl)naphthalene (1g)

${ }^{1} \mathbf{H}$ NMR $\left(600 \mathrm{MHz}, \mathrm{CDCl}_{3}\right)$

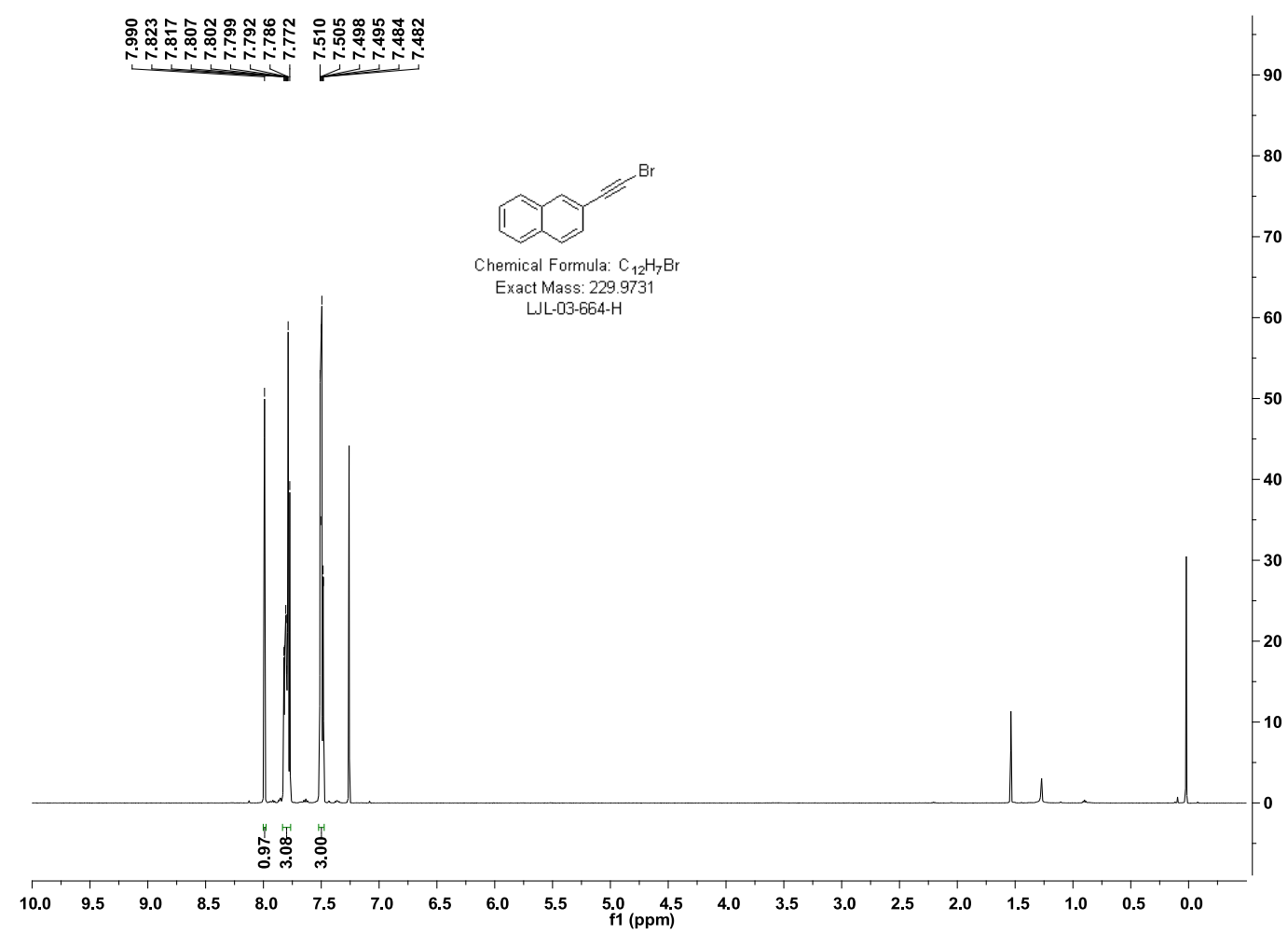

1-(Bromoethynyl)-4-chlorobenzene (1h)

${ }^{1} \mathbf{H}$ NMR $\left(600 \mathrm{MHz}, \mathrm{CDCl}_{3}\right)$

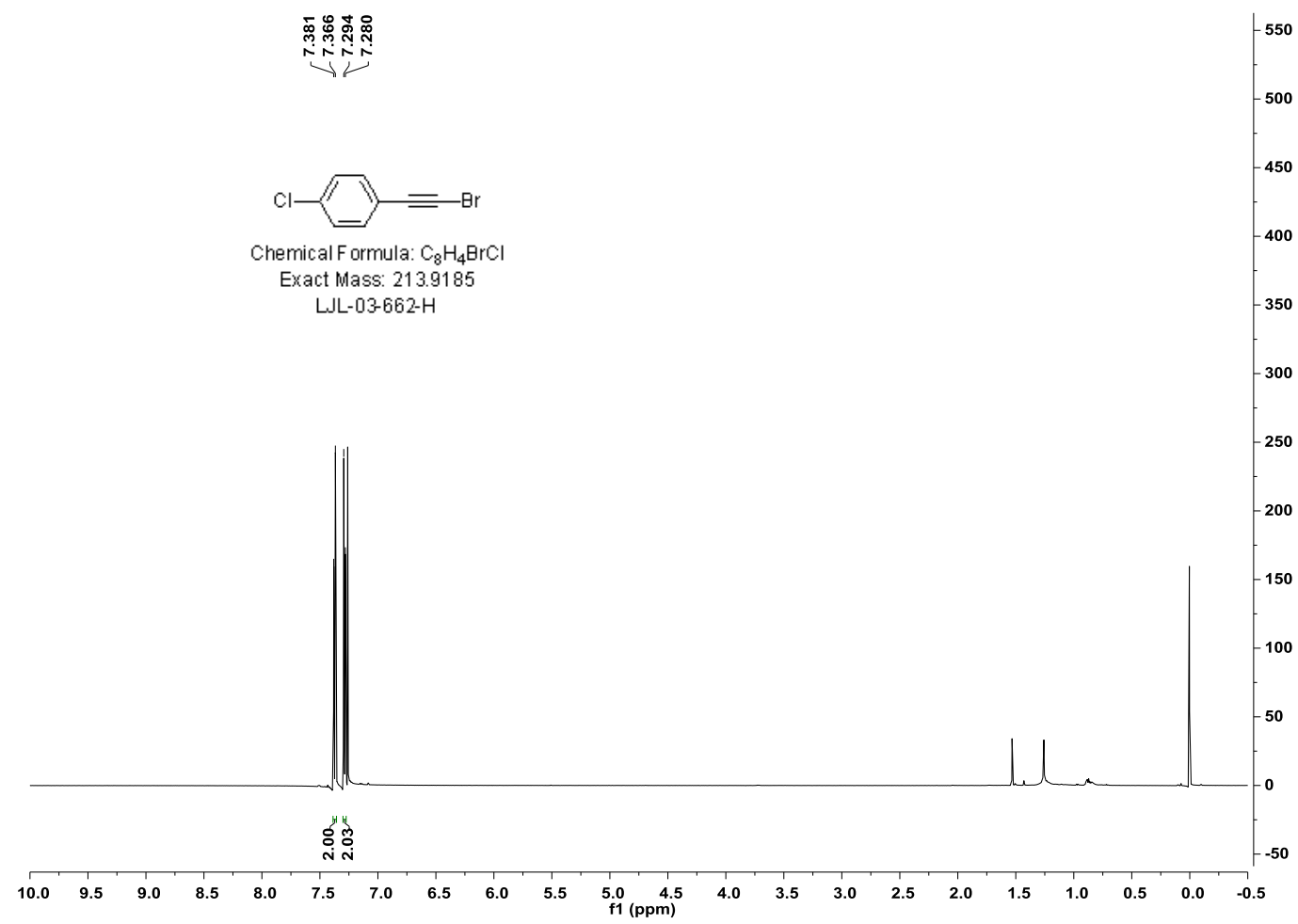


Methyl 4-(bromoethynyl)benzoate (1i)

${ }^{1} \mathbf{H}$ NMR (600 MHz, $\left.\mathrm{CDCl}_{3}\right)$

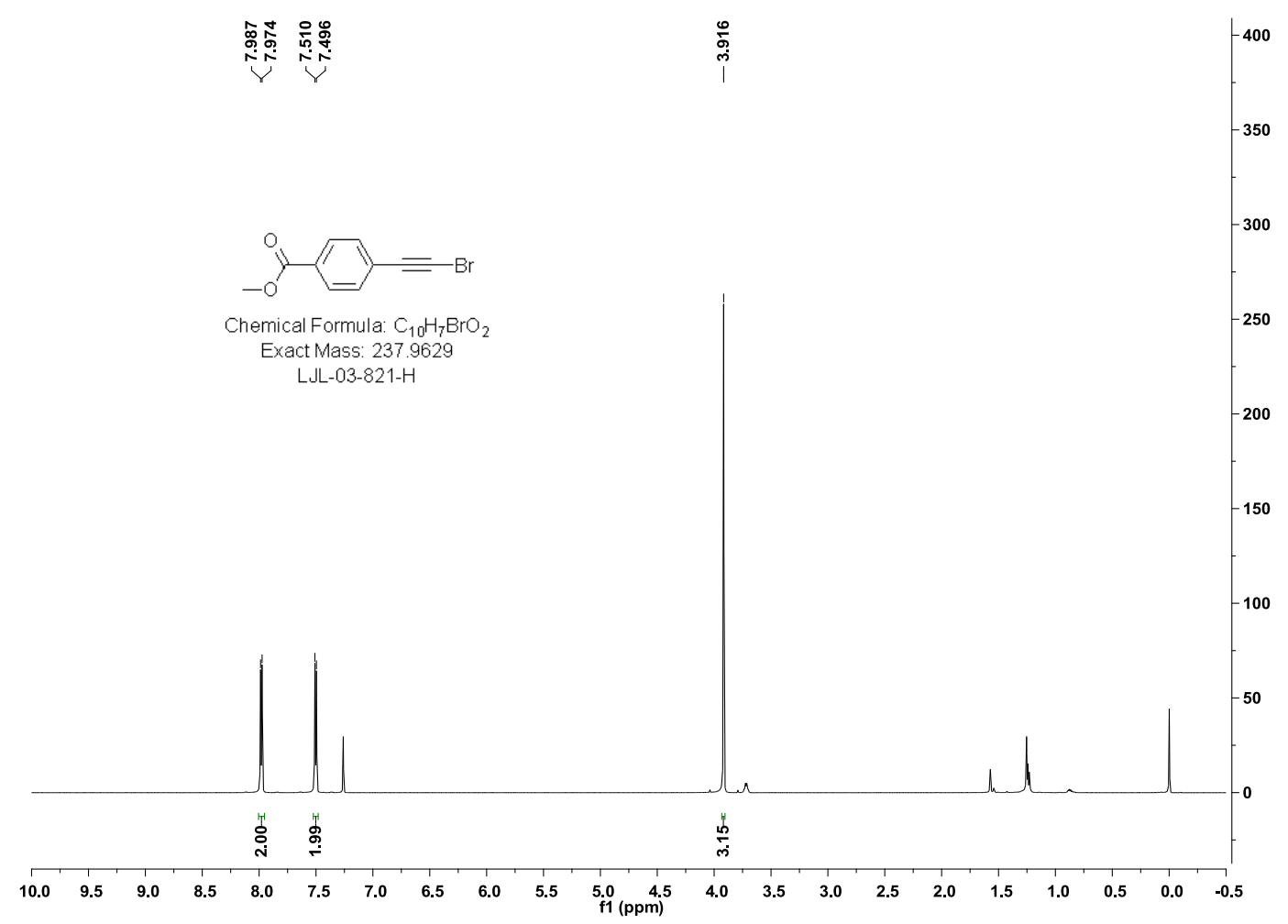

1-3-(Bromoethynyl)thiophene) (1j)

${ }^{1} \mathbf{H}$ NMR (600 MHz, $\left.\mathrm{CDCl}_{3}\right)$

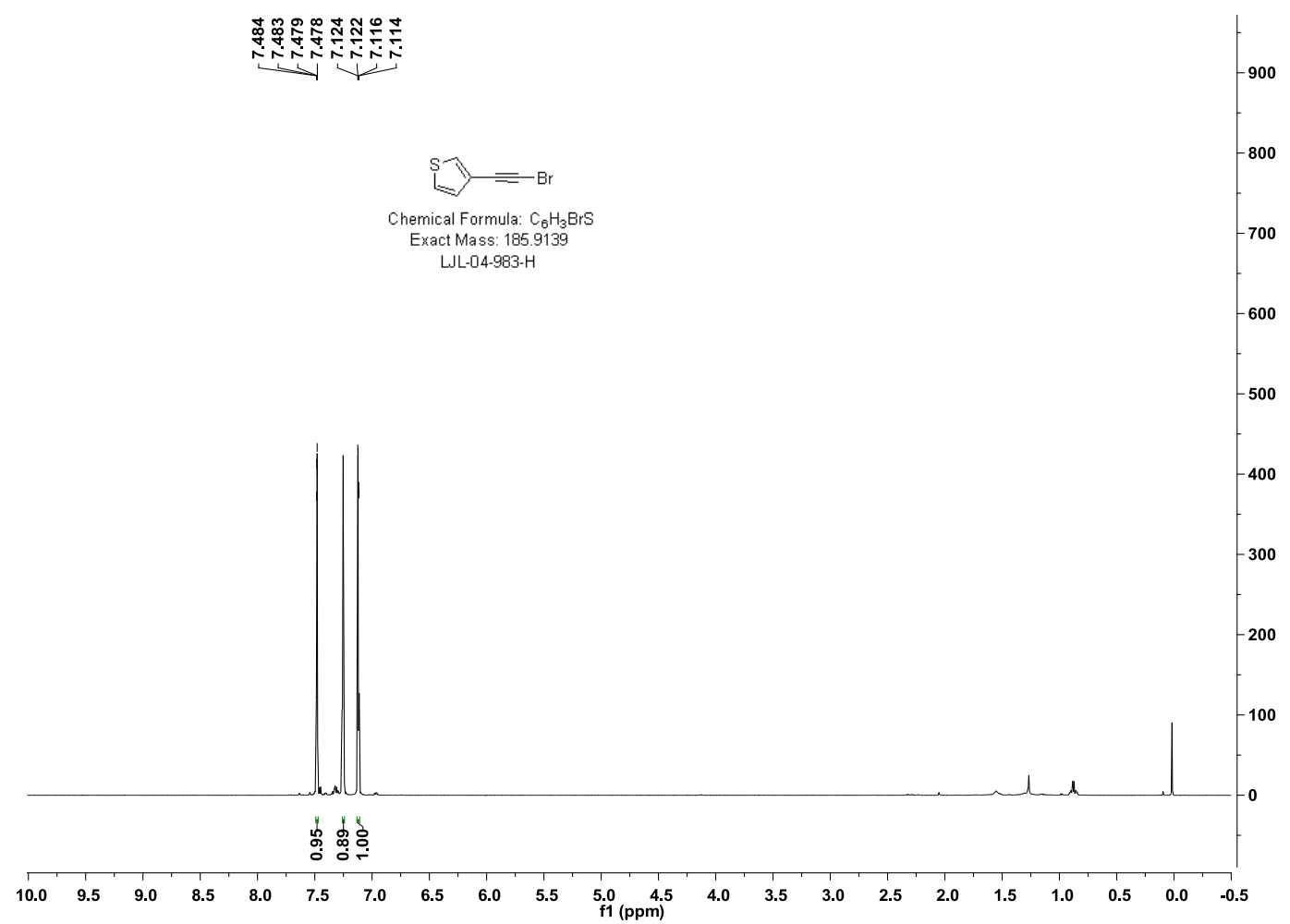


3-(Bromoethynyl)pyridine (1k)

${ }^{1} \mathbf{H}$ NMR (600 MHz, $\left.\mathrm{CDCl}_{3}\right)$

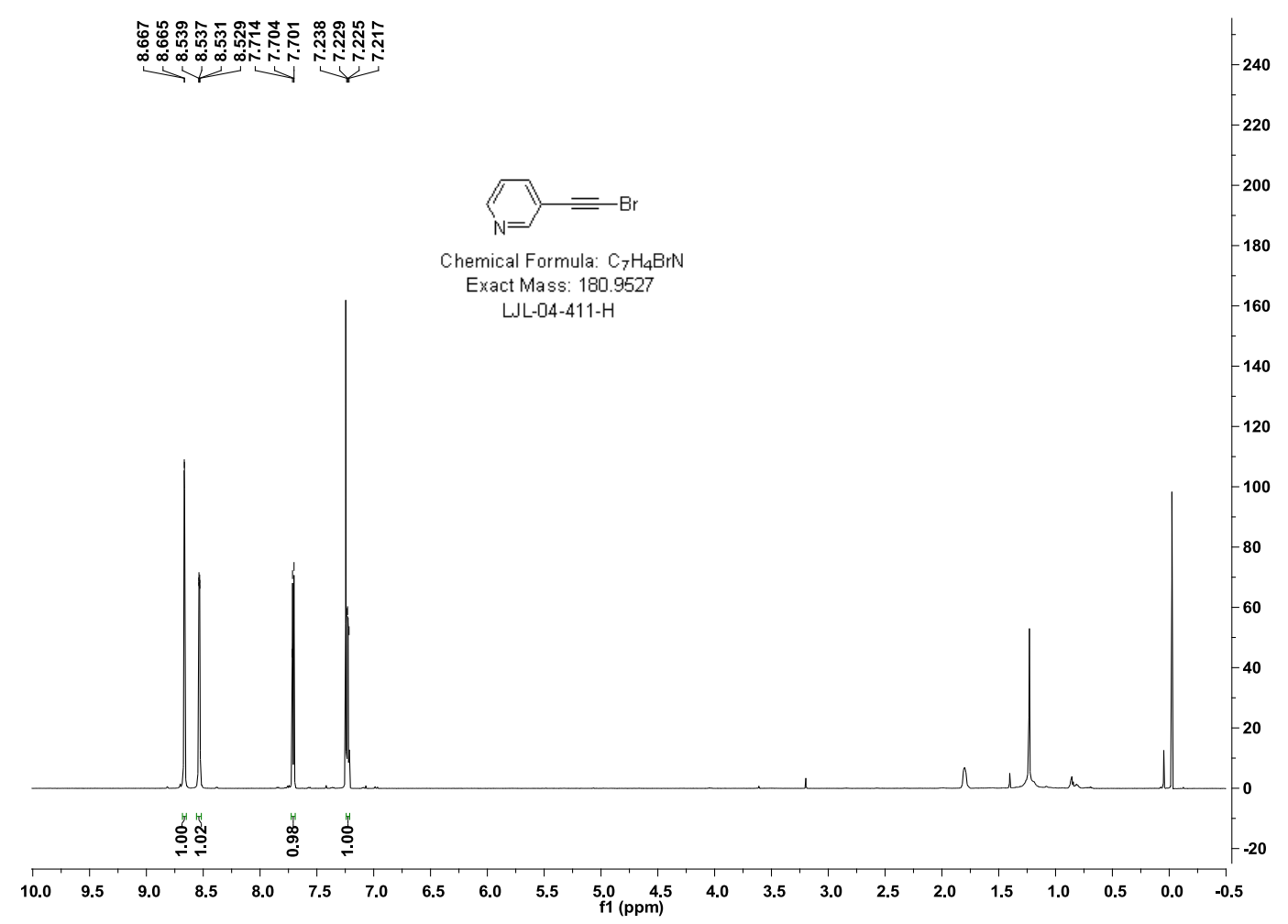

1-Bromododec-1-yne (11)

${ }^{1} \mathbf{H}$ NMR $\left(600 \mathrm{MHz}, \mathrm{CDCl}_{3}\right)$

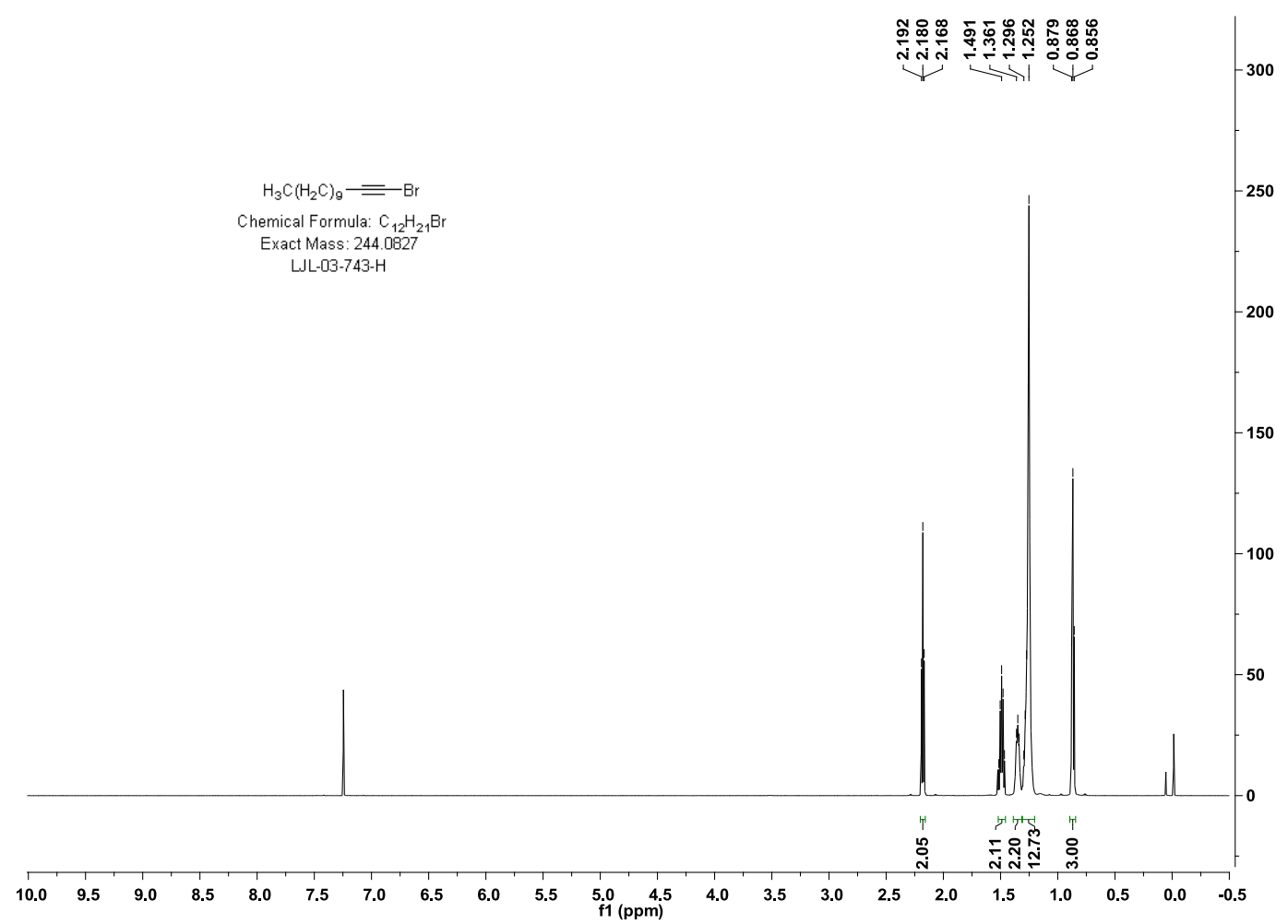


((5-Bromopent-4-yn-1-yl)oxy)(tert-butyl)dimethylsilane (1 m)

${ }^{1} \mathbf{H}$ NMR (600 MHz, $\left.\mathrm{CDCl}_{3}\right)$

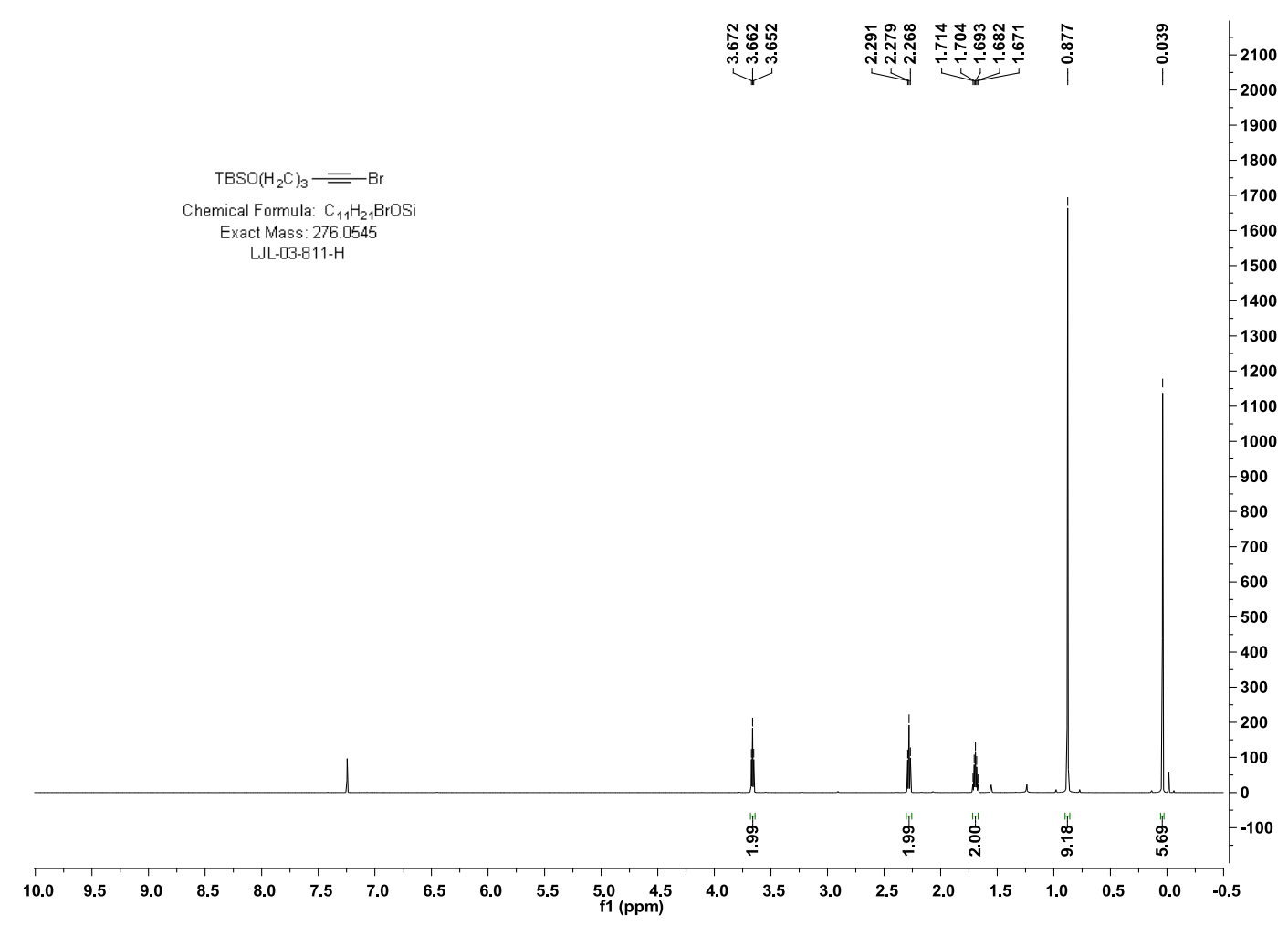

5-Bromopent-4-yn-1-yl acetate (1n)

${ }^{1} \mathbf{H}$ NMR (600 MHz, $\left.\mathrm{CDCl}_{3}\right)$

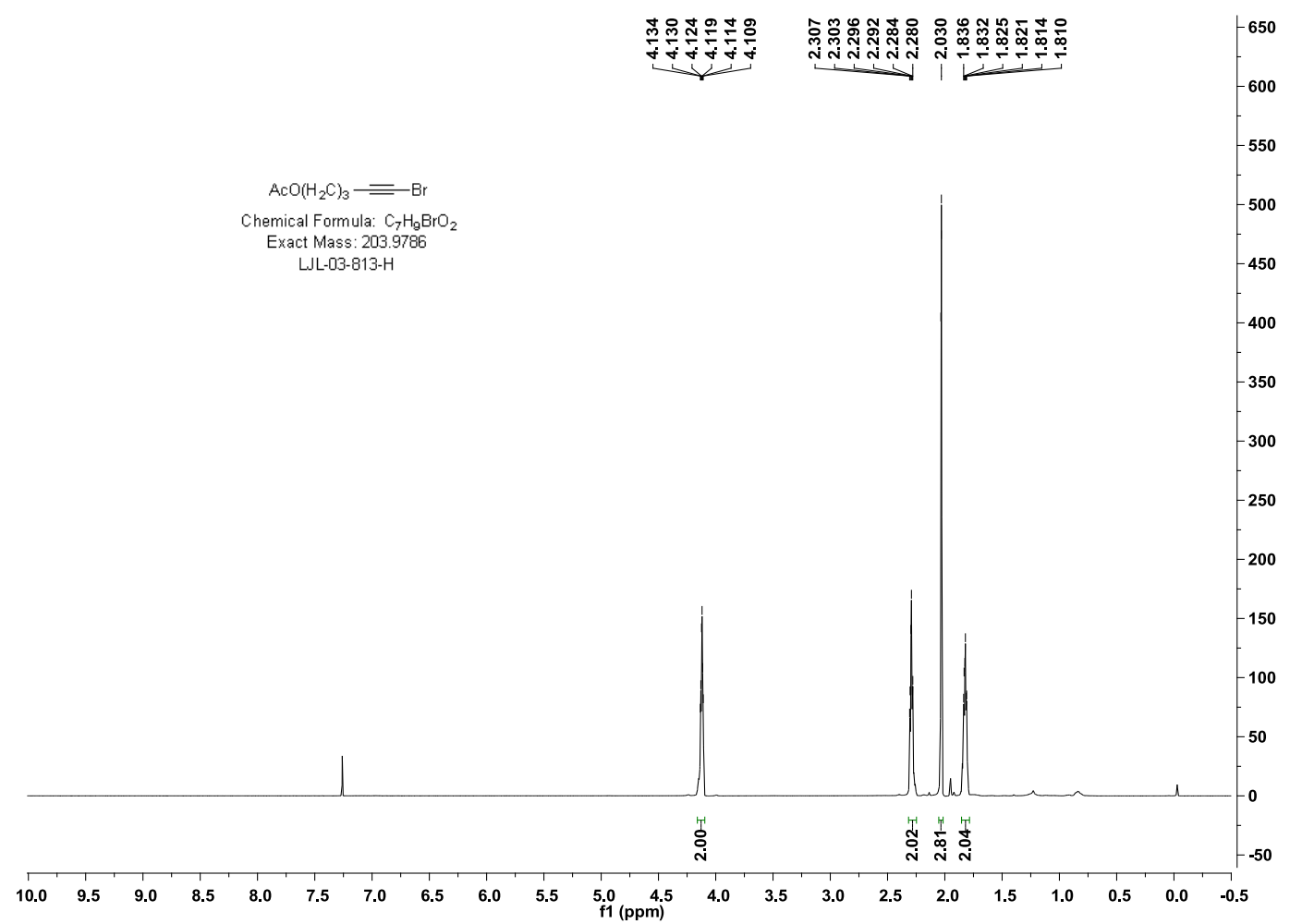


5-Bromopent-4-yn-1-yl 4-cyanobenzoate (10)

${ }^{1} \mathbf{H}$ NMR $\left(600 \mathrm{MHz}, \mathrm{CDCl}_{3}\right)$

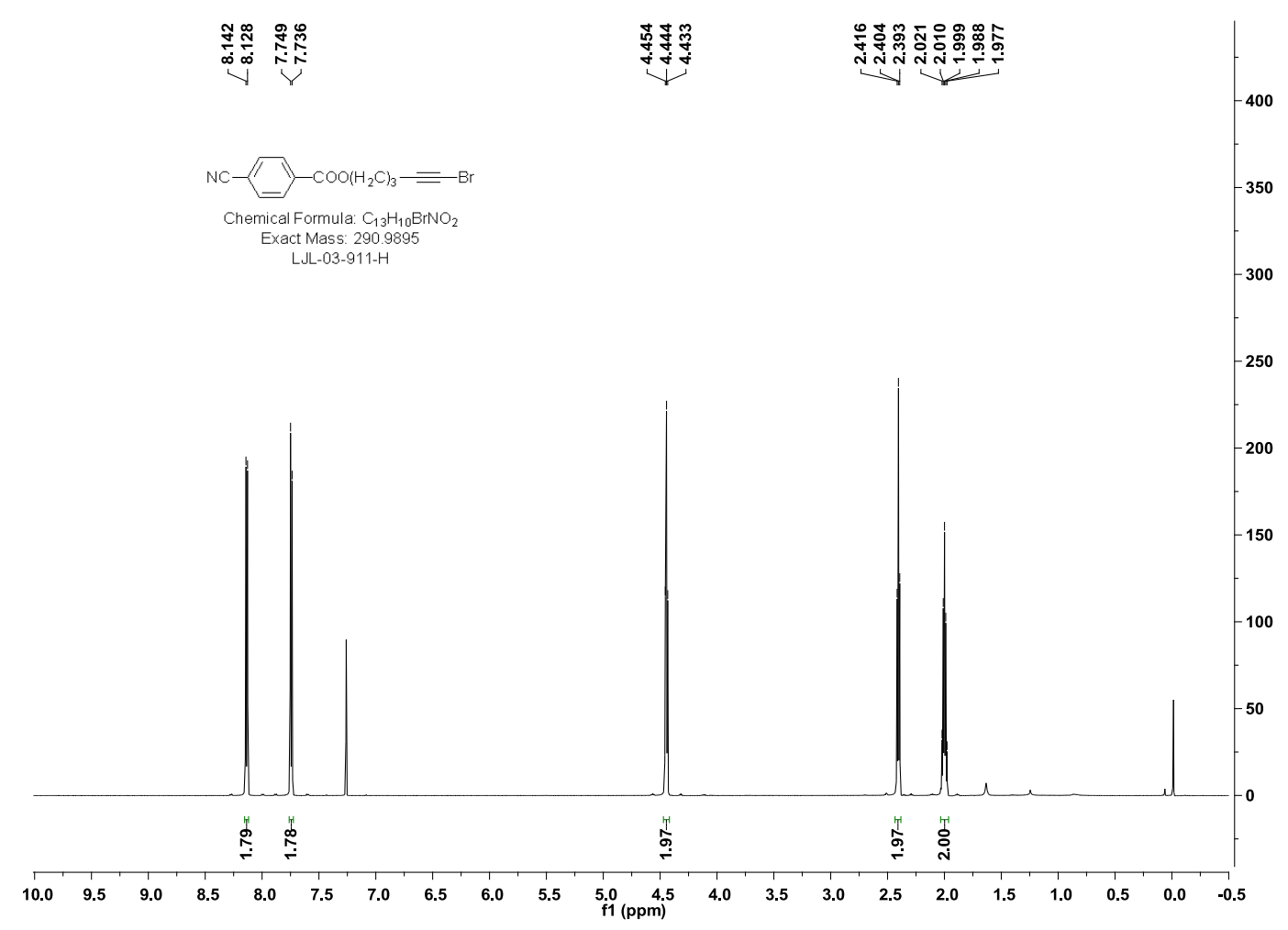

${ }^{13}$ C NMR (150.8 MHz, $\left.\mathrm{CDCl}_{3}\right)$

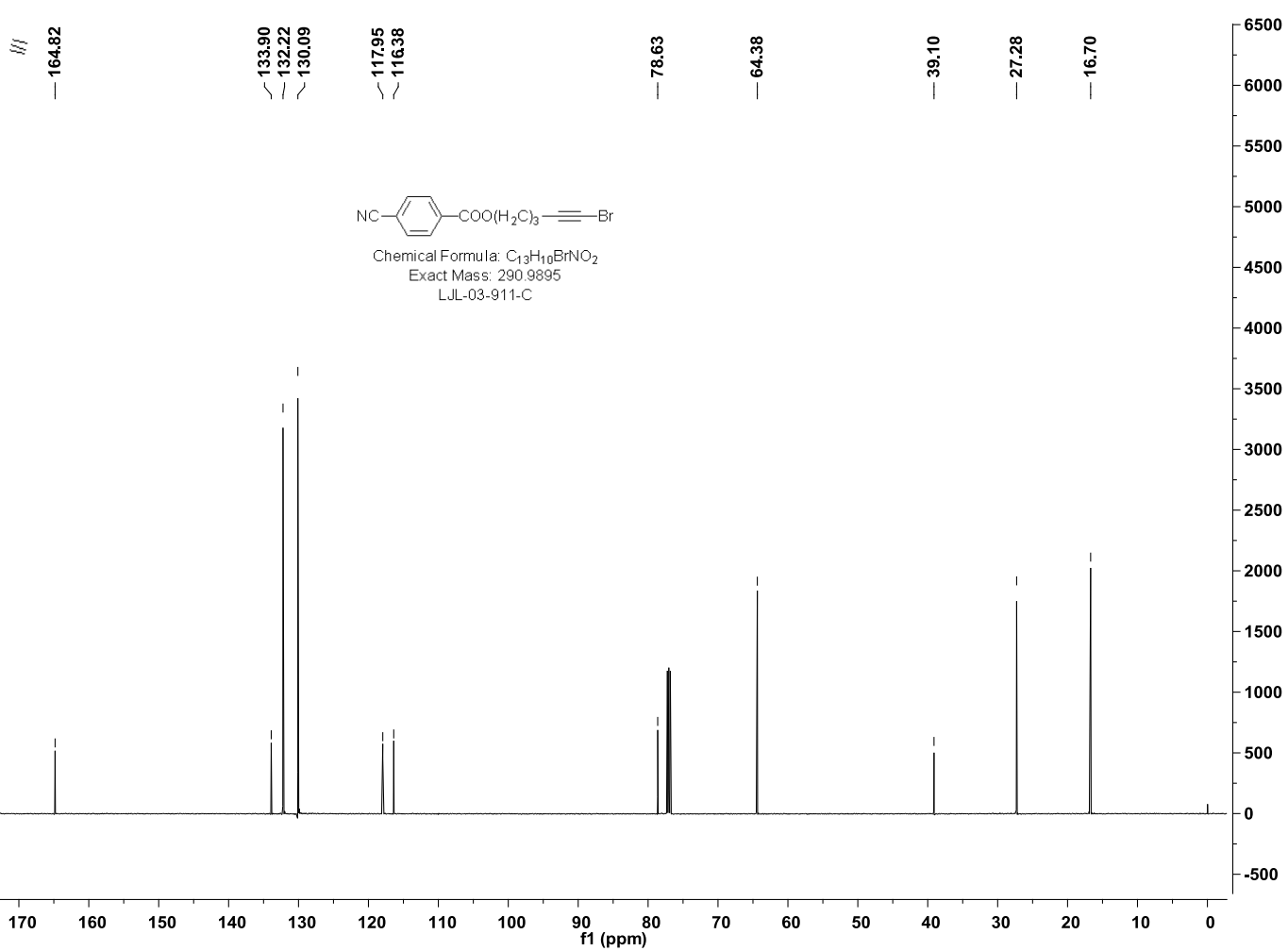


5-Bromopent-4-yn-1-yl 4-nitrobenzoate (1p)

${ }^{1} \mathbf{H}$ NMR (600 MHz, $\left.\mathrm{CDCl}_{3}\right)$

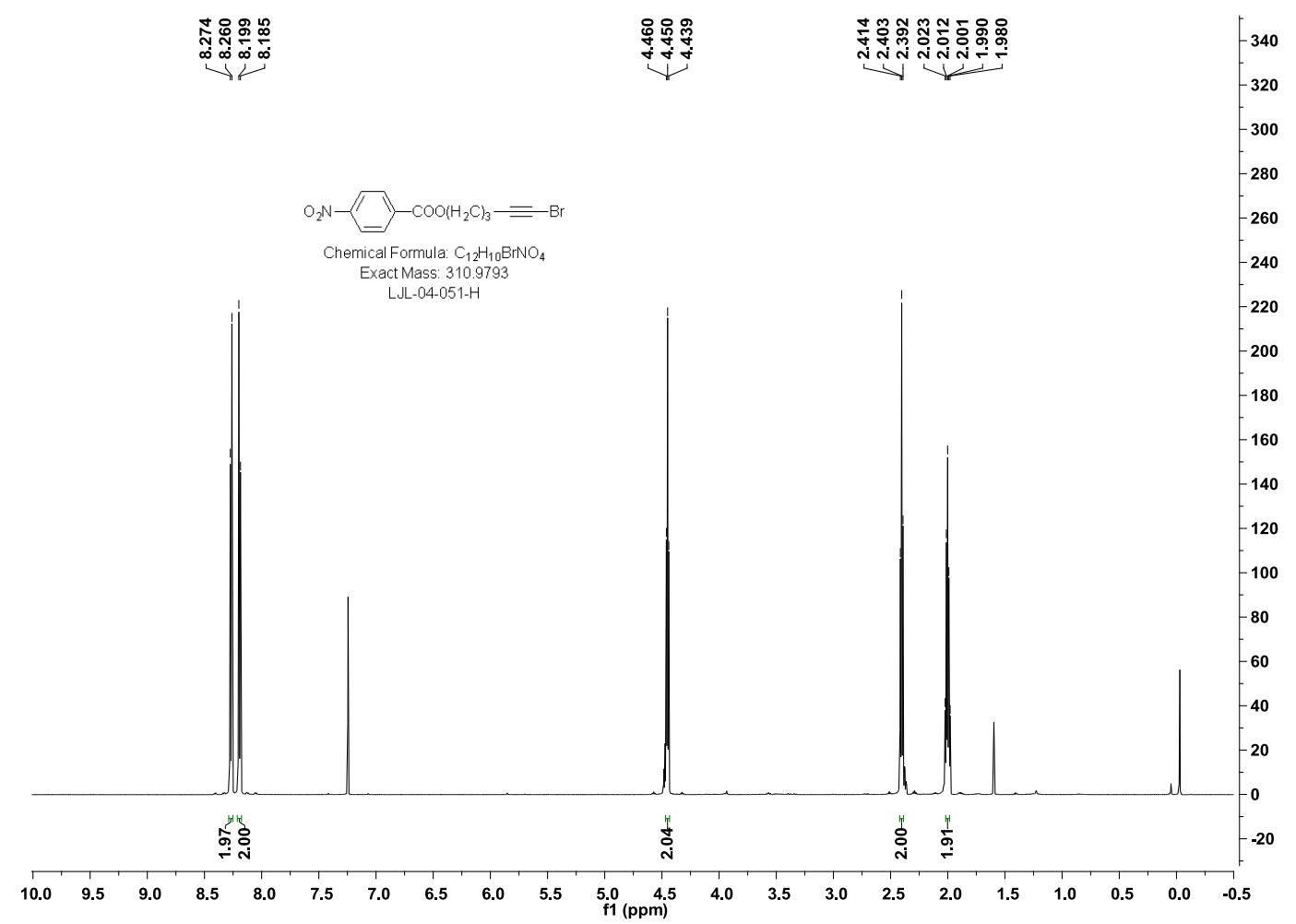

${ }^{13}$ C NMR (150.8 MHz, $\left.\mathrm{CDCl}_{3}\right)$

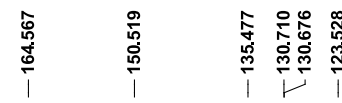

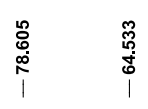

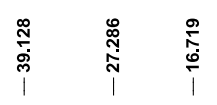

$-4000$

3500

$\mathrm{O}_{2} \mathrm{~N}-\longrightarrow-\mathrm{COO}_{2} \mathrm{H}_{2} \mathrm{C}_{3}=\mathrm{Br}$

Chemical Formula: $\mathrm{C}_{12} \mathrm{H}_{10} \mathrm{BrNO}_{4}$

Exact Mass: 310.9793
LJL-04-051-C

3000

2500

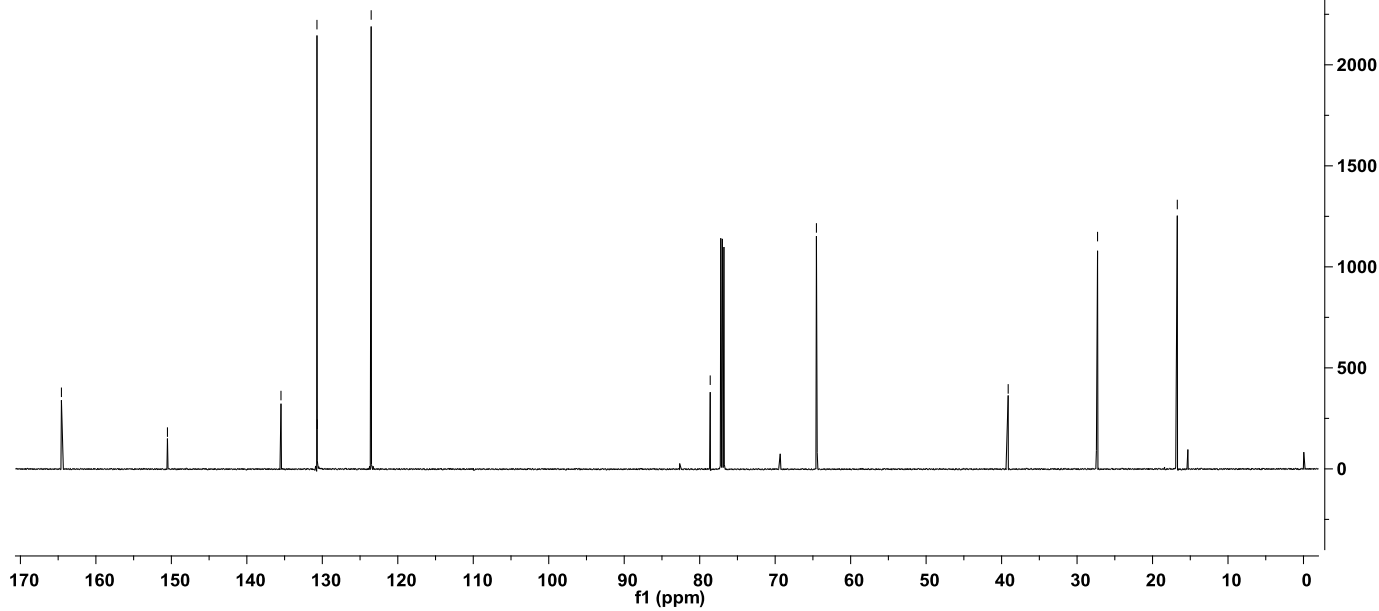


5-Bromopent-4-yn-1-yl diethyl phosphate (1q)

${ }^{1} \mathbf{H}$ NMR (600 MHz, $\left.\mathrm{CDCl}_{3}\right)$

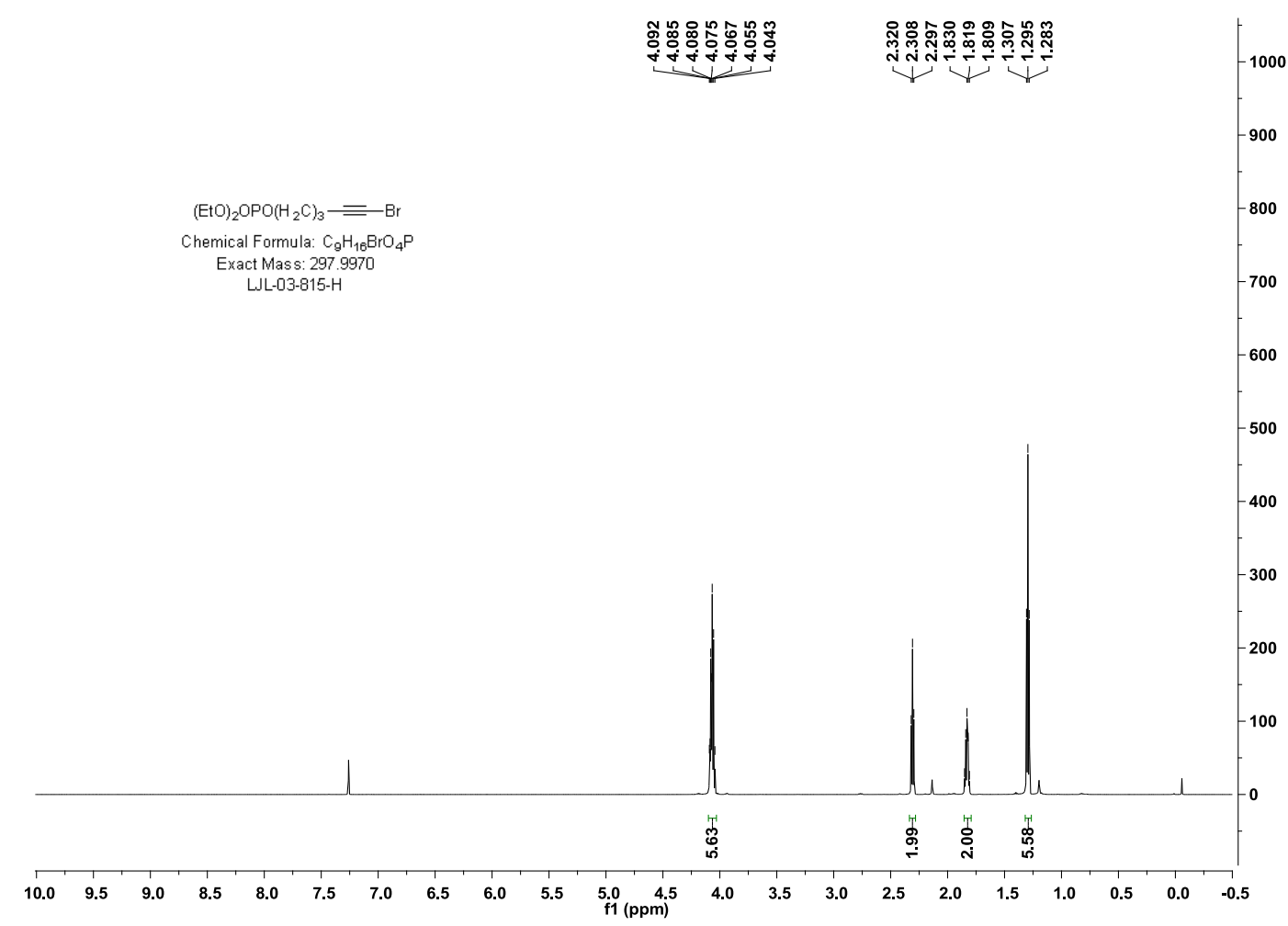

${ }^{13}$ C NMR (150.8 MHz, $\left.\mathrm{CDCl}_{3}\right)$

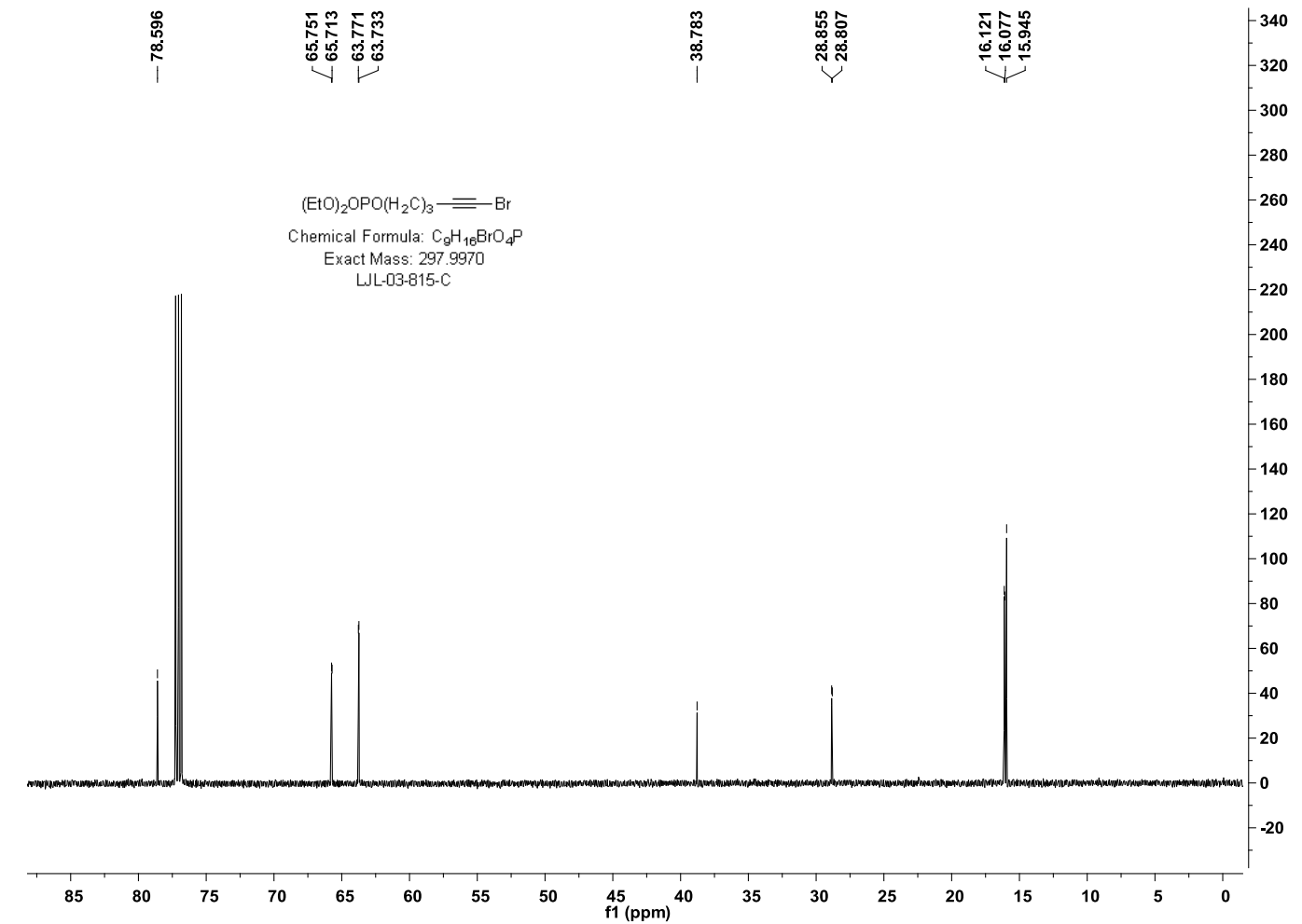


5-Bromopent-4-yn-1-yl 4-methylbenzenesulfonate (1r)

${ }^{1} \mathbf{H}$ NMR $\left(600 \mathrm{MHz}, \mathrm{CDCl}_{3}\right)$

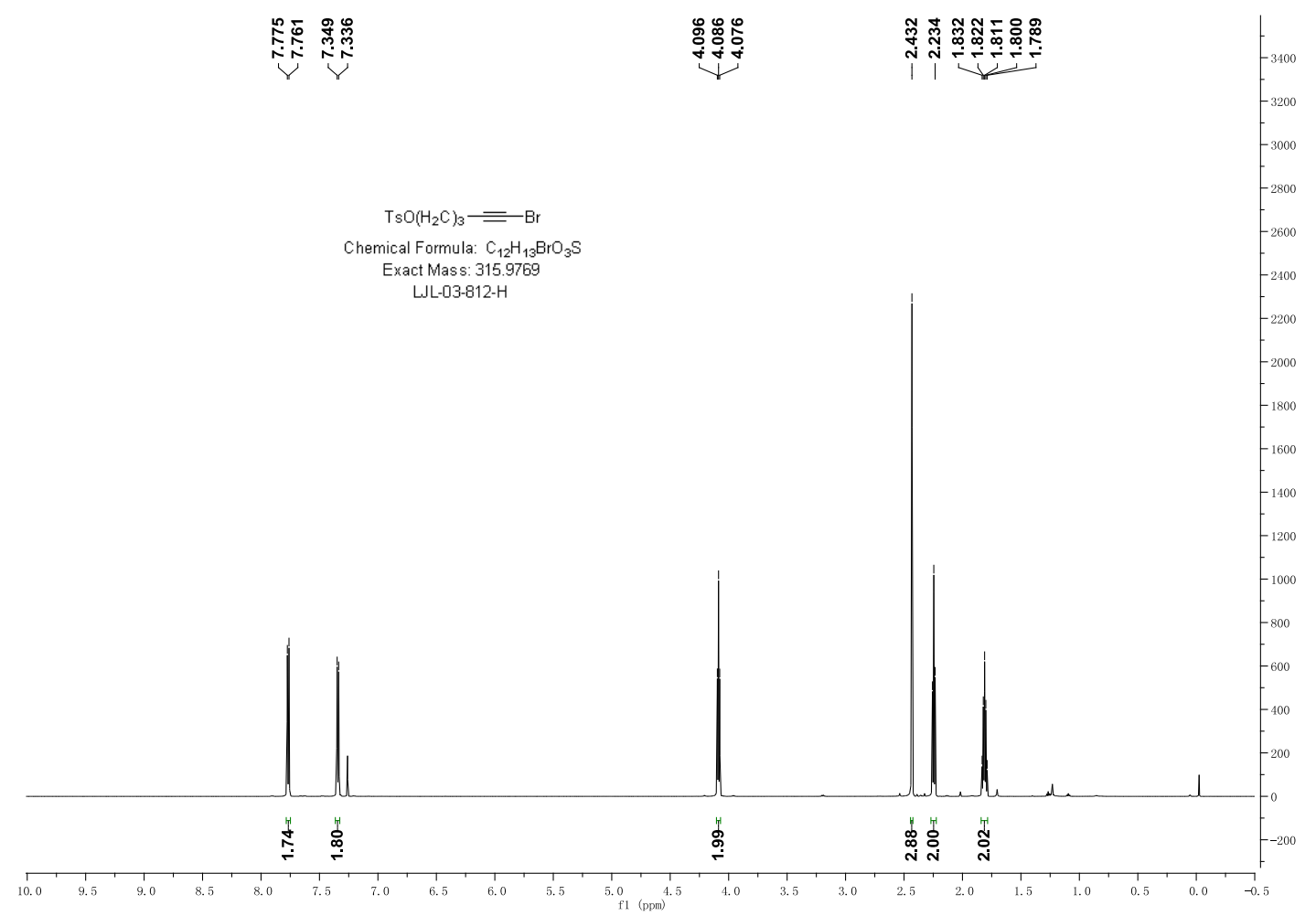

(8R,9S,13S,14S)-3-((3-bromoprop-2-yn-1-yl)oxy)-13-methyl-

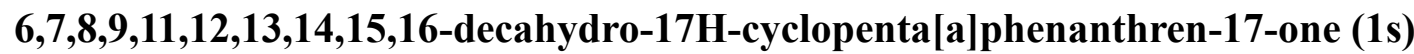
${ }^{1} \mathbf{H}$ NMR $\left(600 \mathrm{MHz}, \mathrm{CDCl}_{3}\right)$

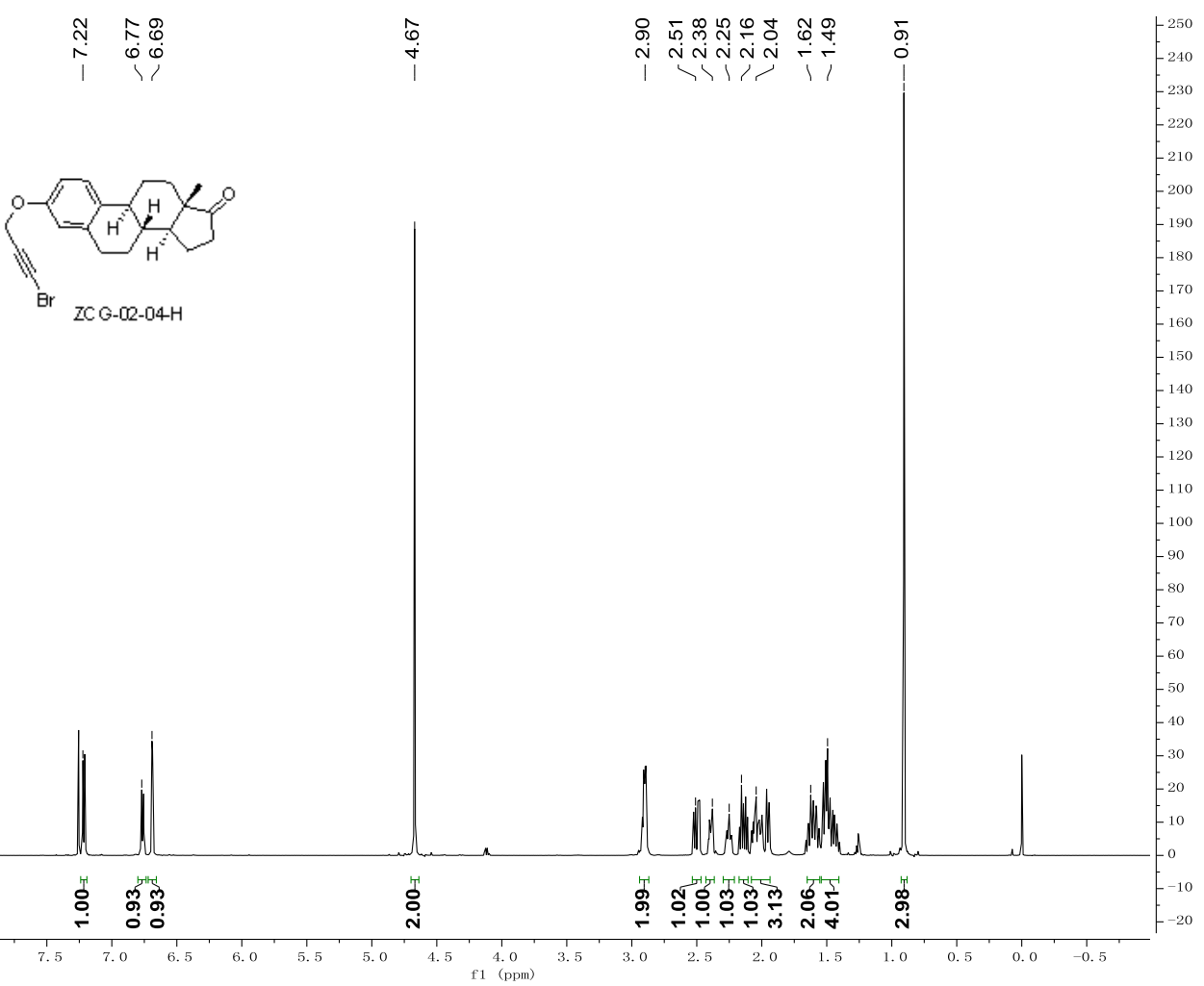




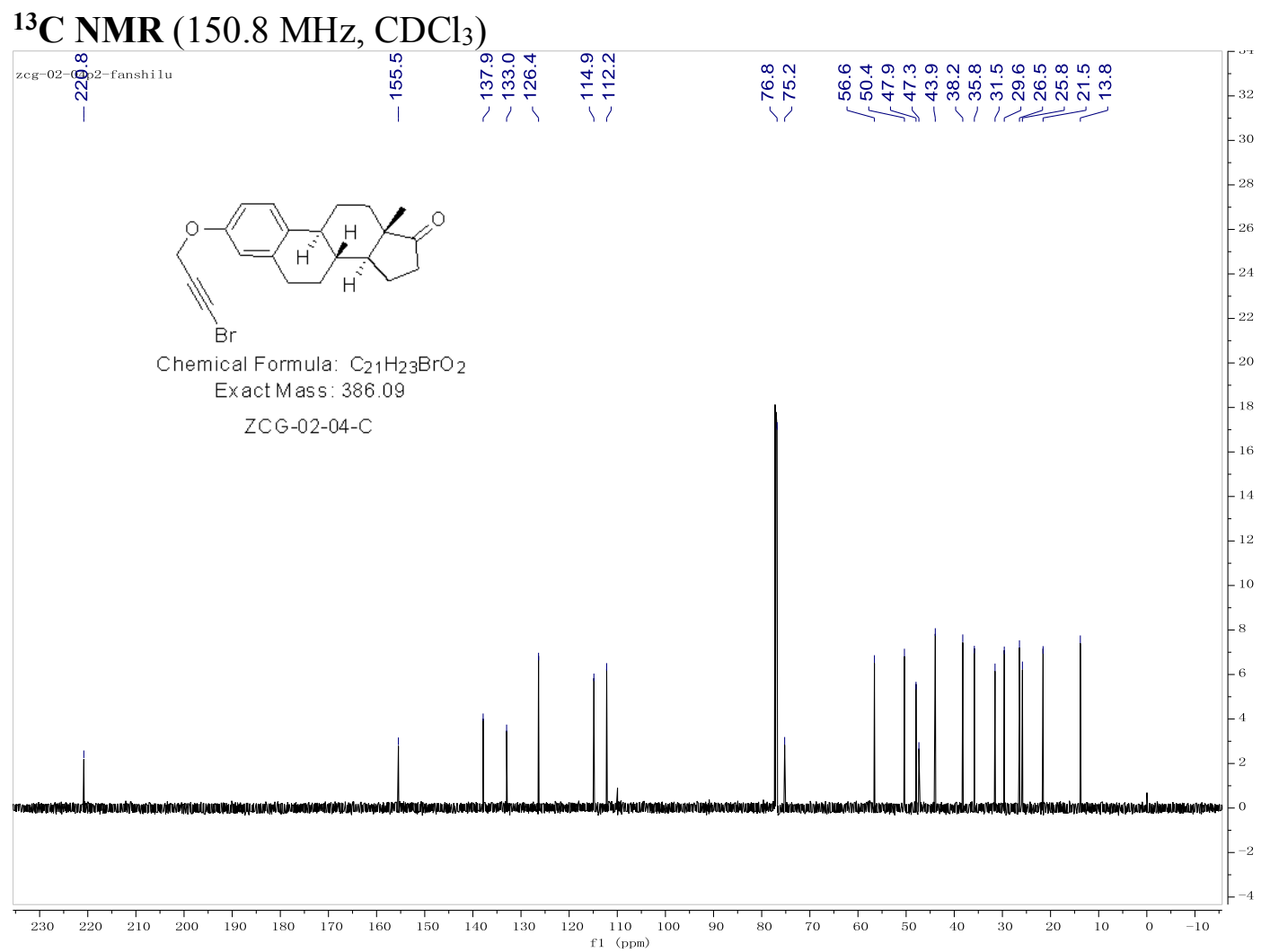

(1S,2R,4R)-2-((3-bromoprop-2-yn-1-yl)oxy)-1-isopropyl-4-methylcyclohexane (1t) ${ }^{1} \mathbf{H}$ NMR (600 MHz, $\left.\mathrm{CDCl}_{3}\right)$

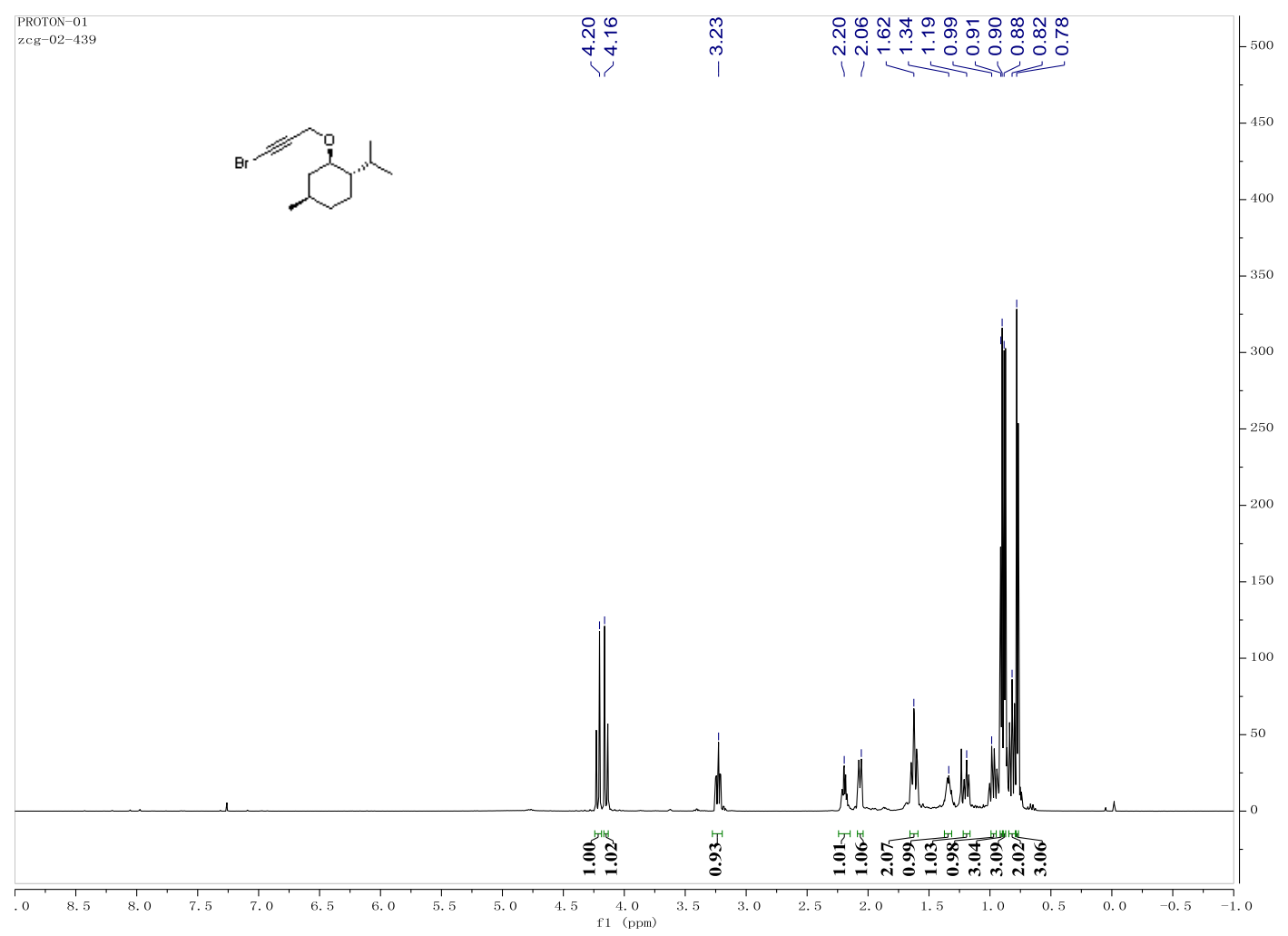




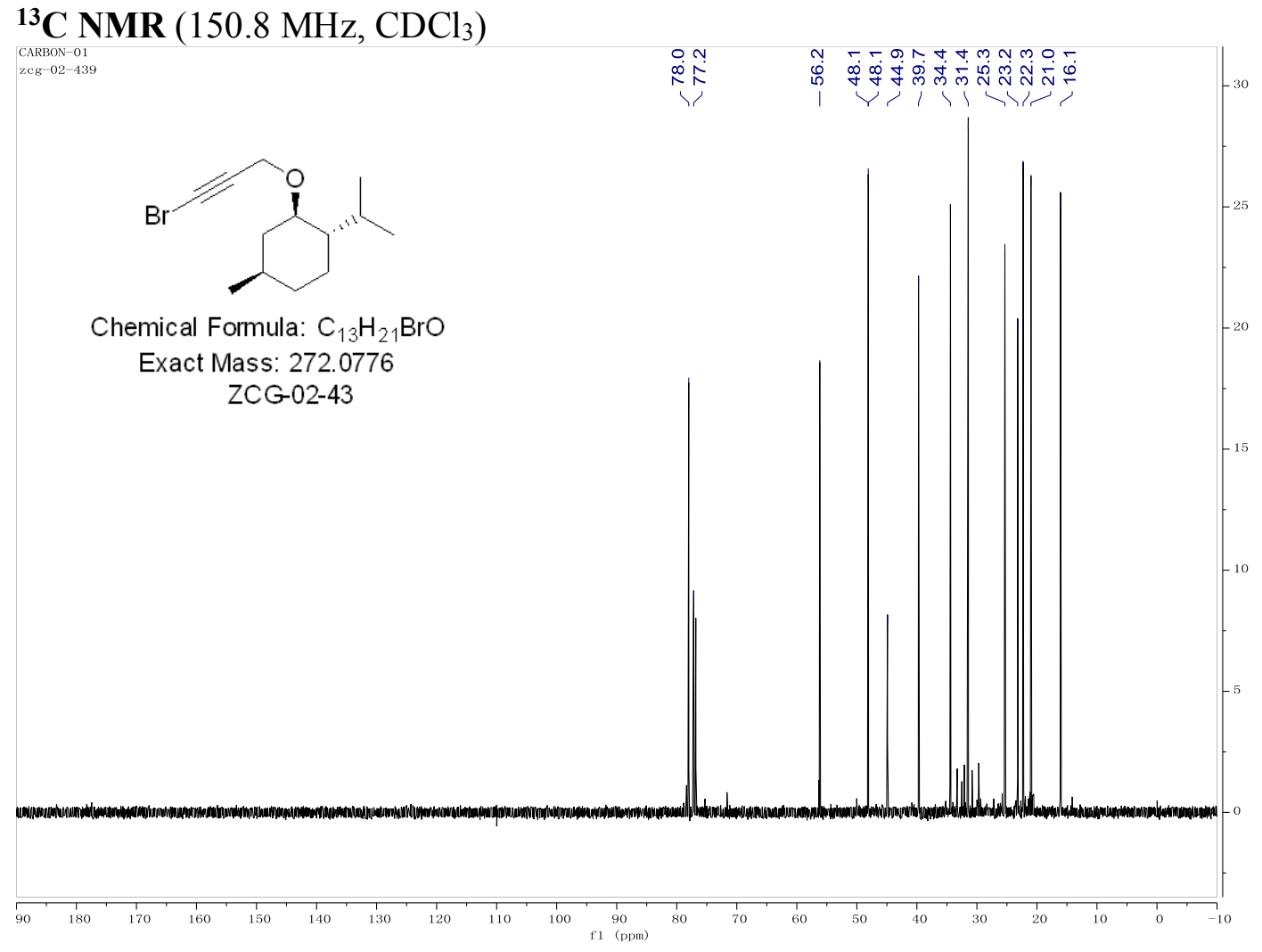

1-(perfluorohex-1-yn-1-yl)-4-propylbenzene (6a)

${ }^{1} \mathbf{H}$ NMR (600 MHz, $\mathrm{CDCl}_{3}$ )

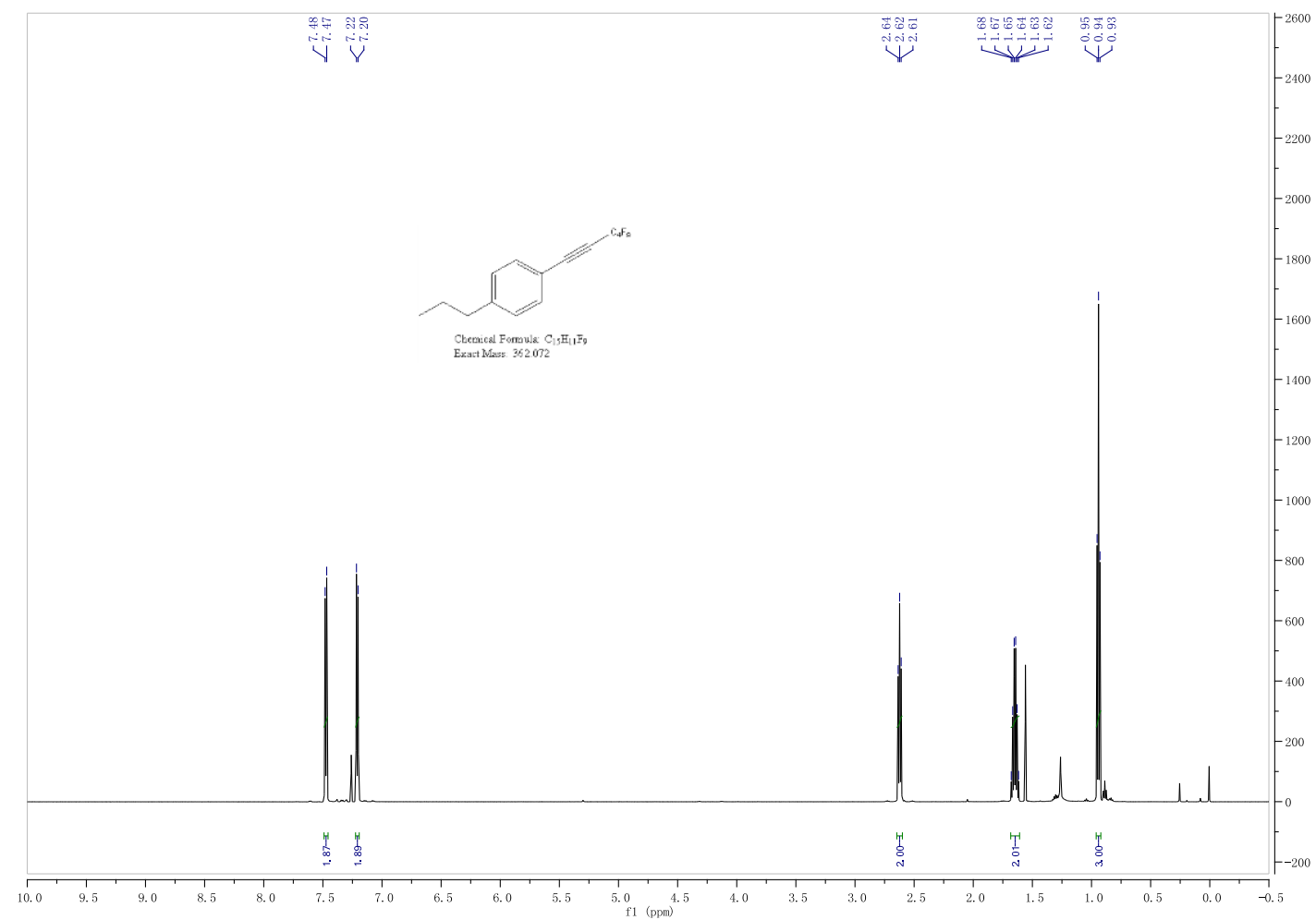


${ }^{19}$ F NMR $\left(564 \mathrm{MHz}, \mathrm{CDCl}_{3}\right)$

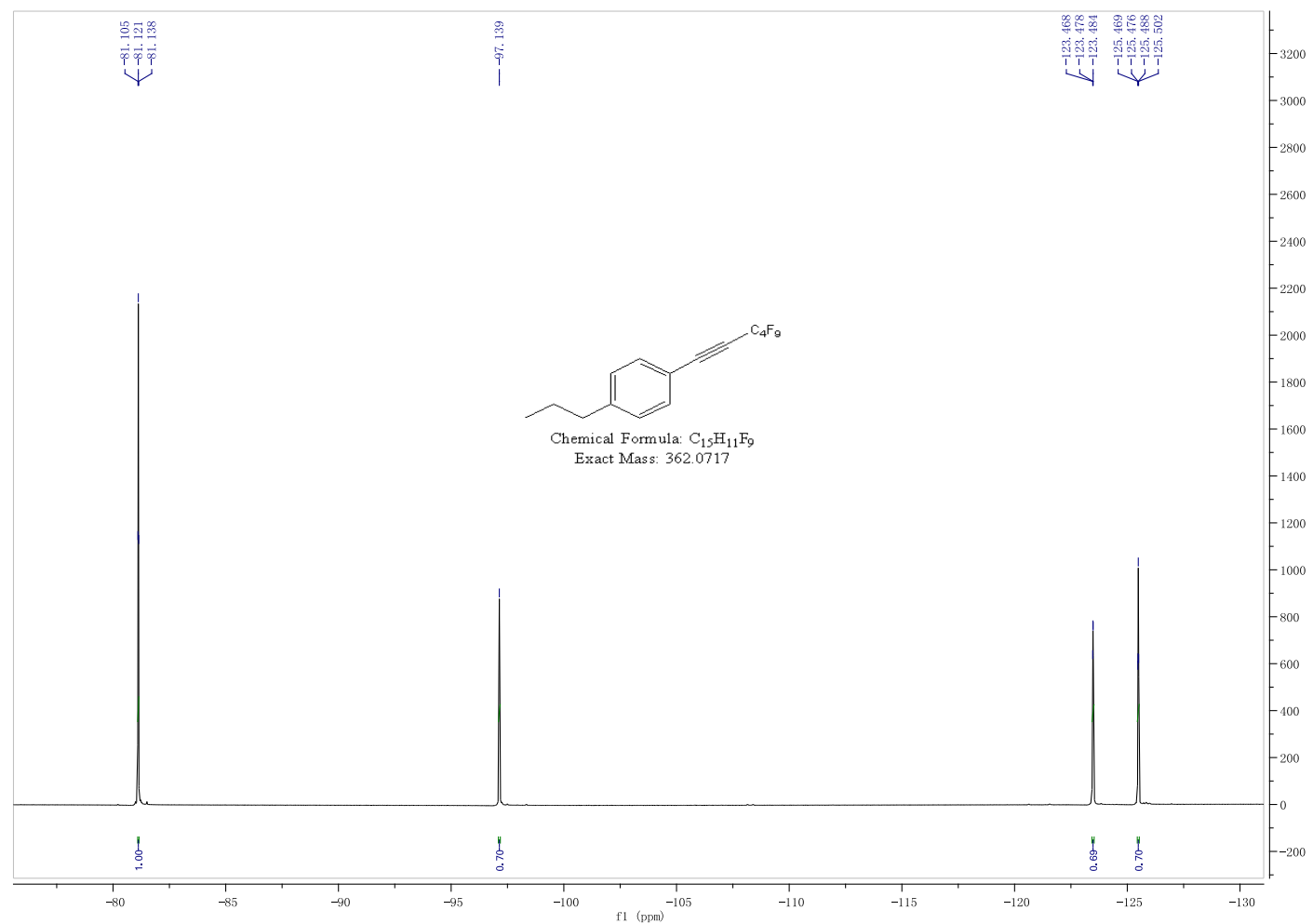

1-pentyl-4-(perfluorohex-1-yn-1-yl)benzene (6b)

${ }^{1} \mathbf{H}$ NMR (600 MHz, $\left.\mathrm{CDCl}_{3}\right)$

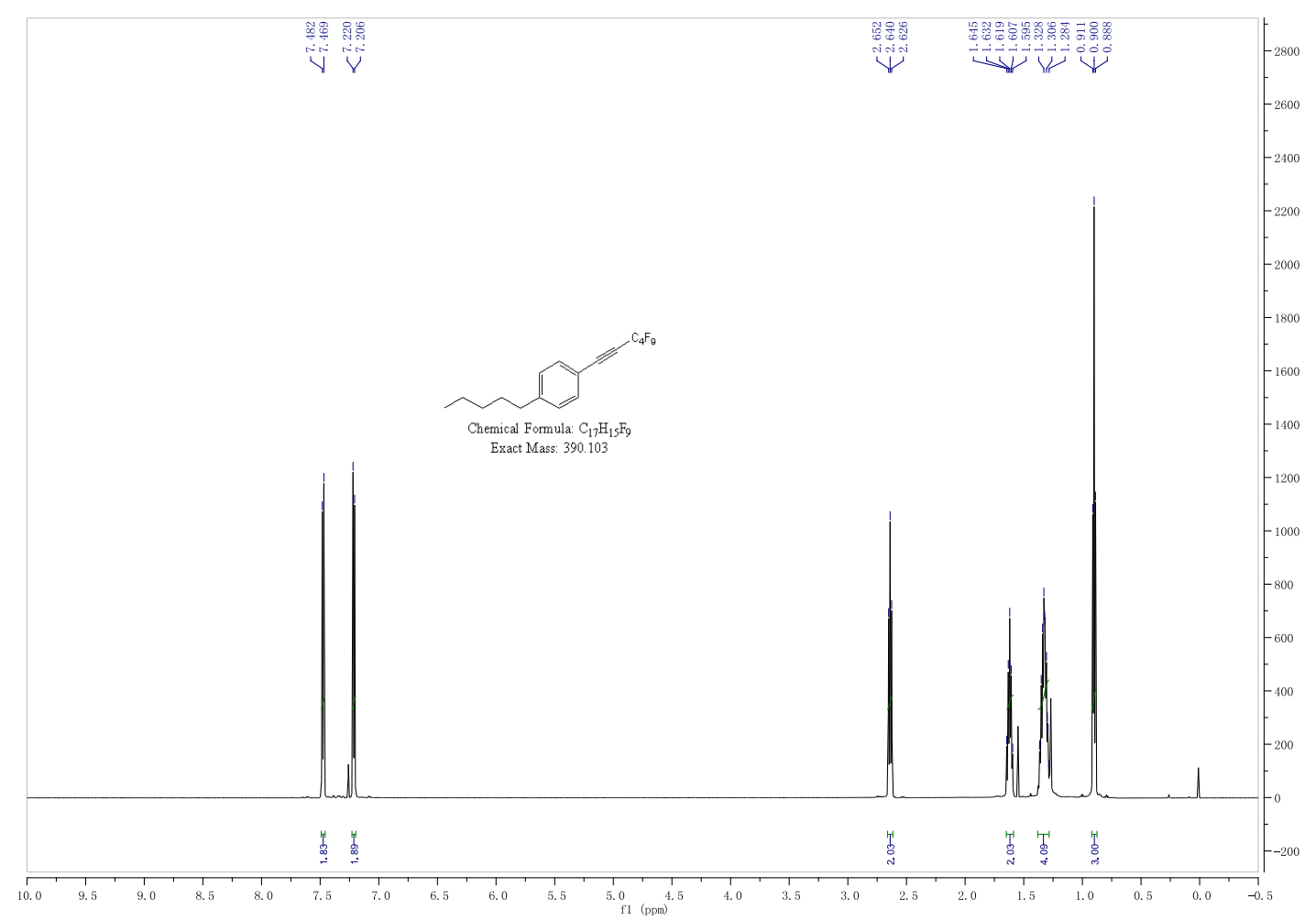


${ }^{19}$ F NMR $\left(564 \mathrm{MHz}, \mathrm{CDCl}_{3}\right)$

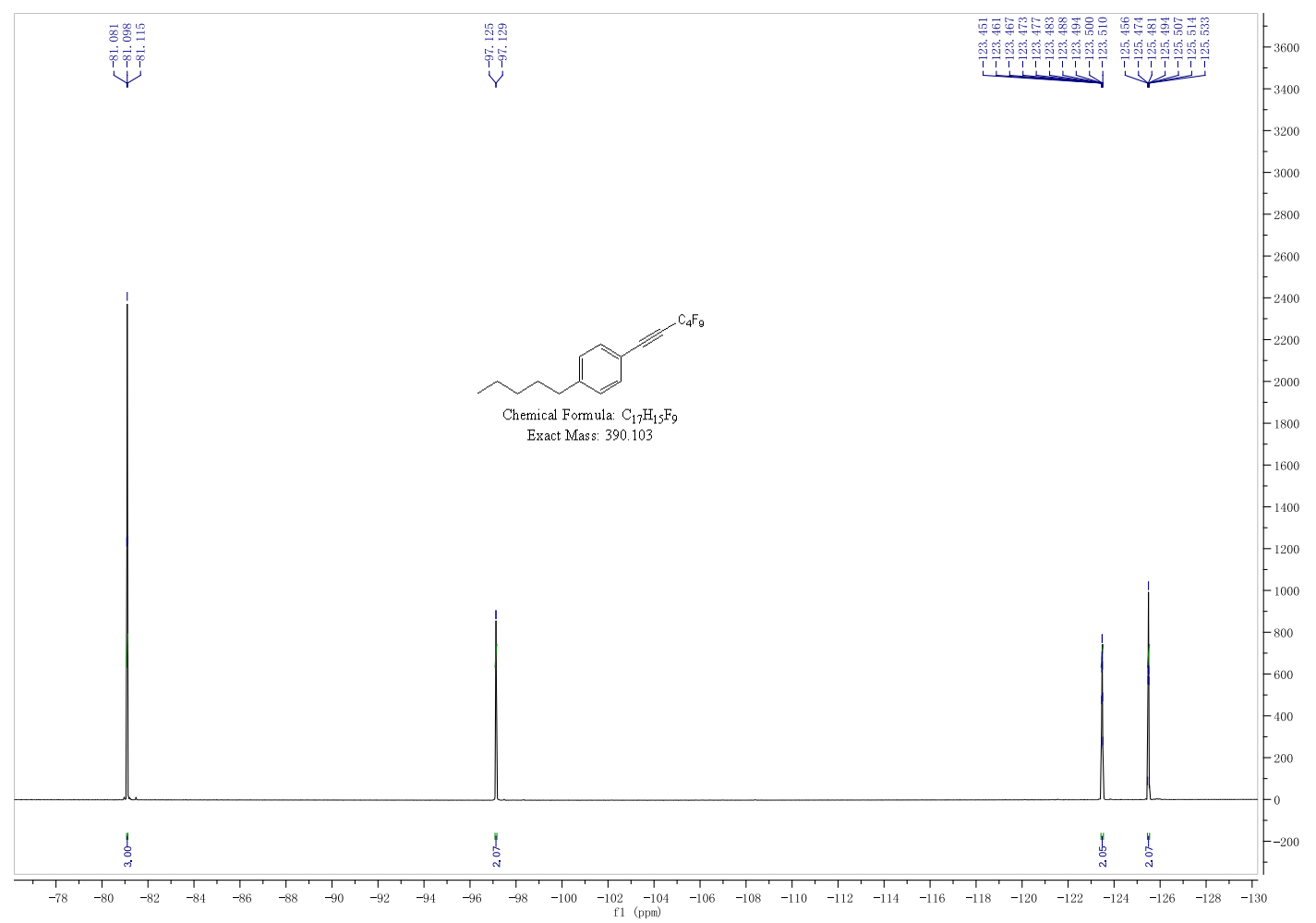

(perfluorohex-1-yn-1-yl)benzene (6c)

${ }^{1} \mathbf{H}$ NMR (600 MHz, $\left.\mathrm{CDCl}_{3}\right)$

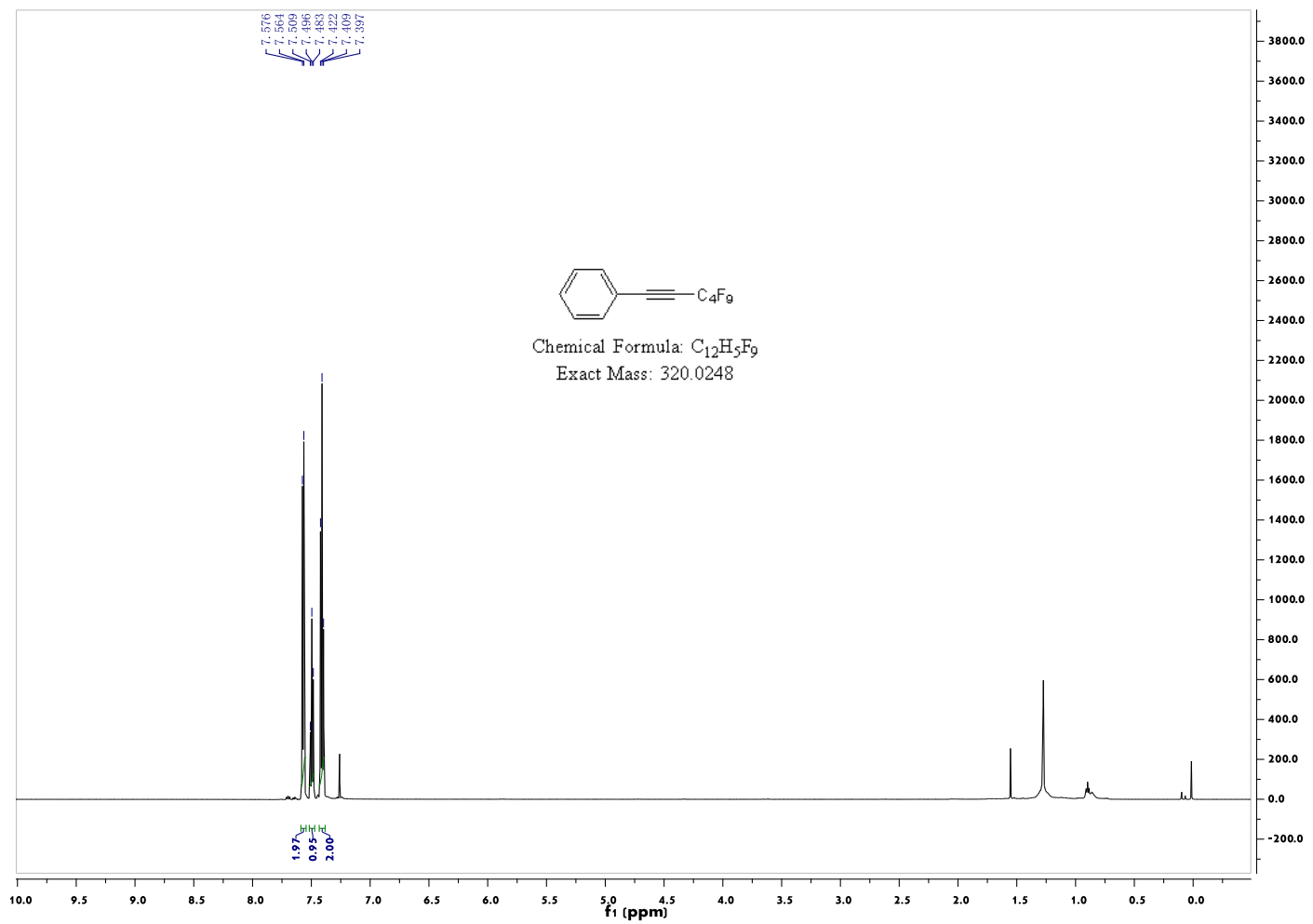


${ }^{19} \mathbf{F}$ NMR $\left(564 \mathrm{MHz}, \mathrm{CDCl}_{3}\right)$

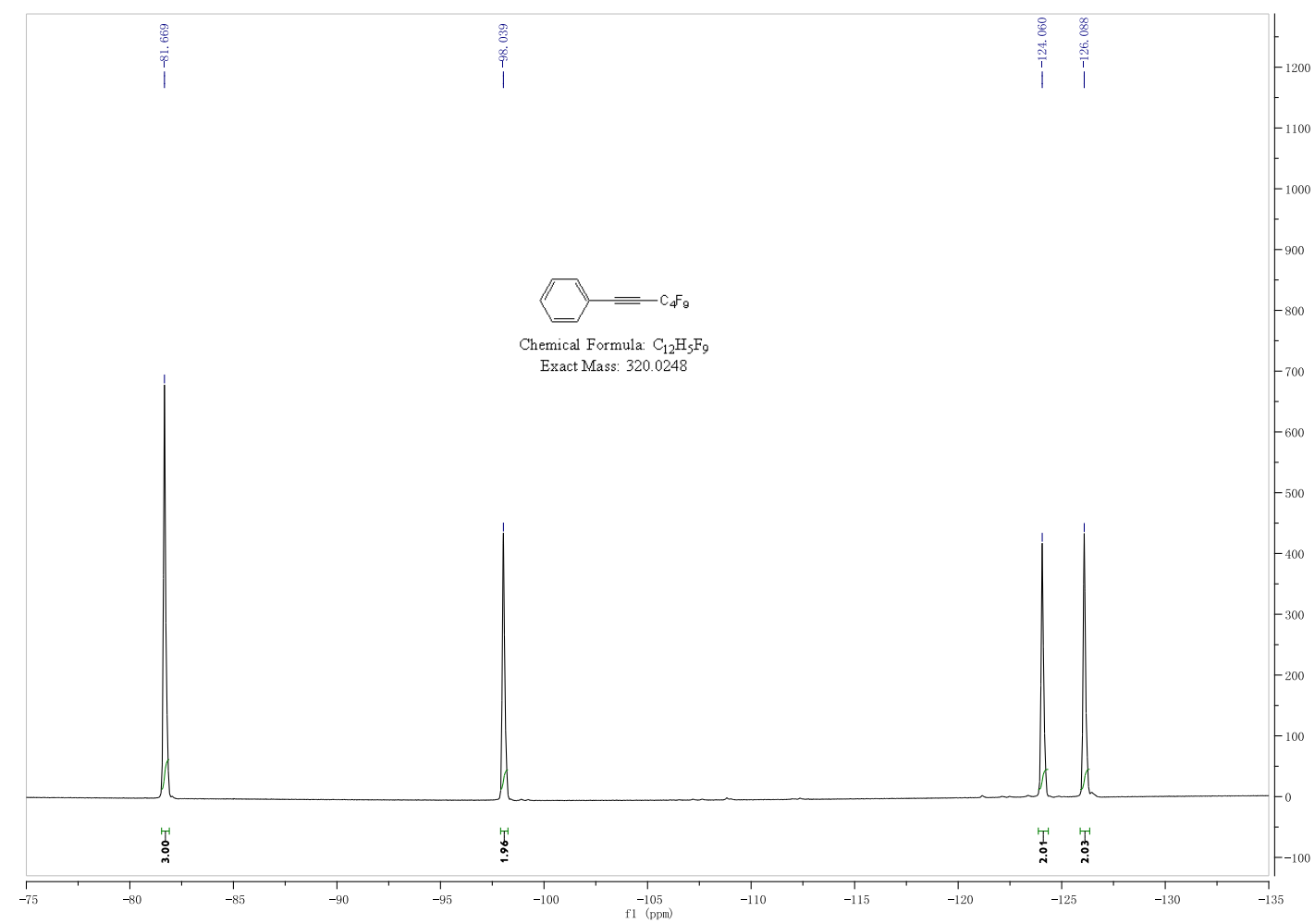

1-methoxy-4-(perfluorohex-1-yn-1-yl)benzene (6d)

${ }^{1} \mathbf{H}$ NMR $\left(600 \mathrm{MHz}, \mathrm{CDCl}_{3}\right)$

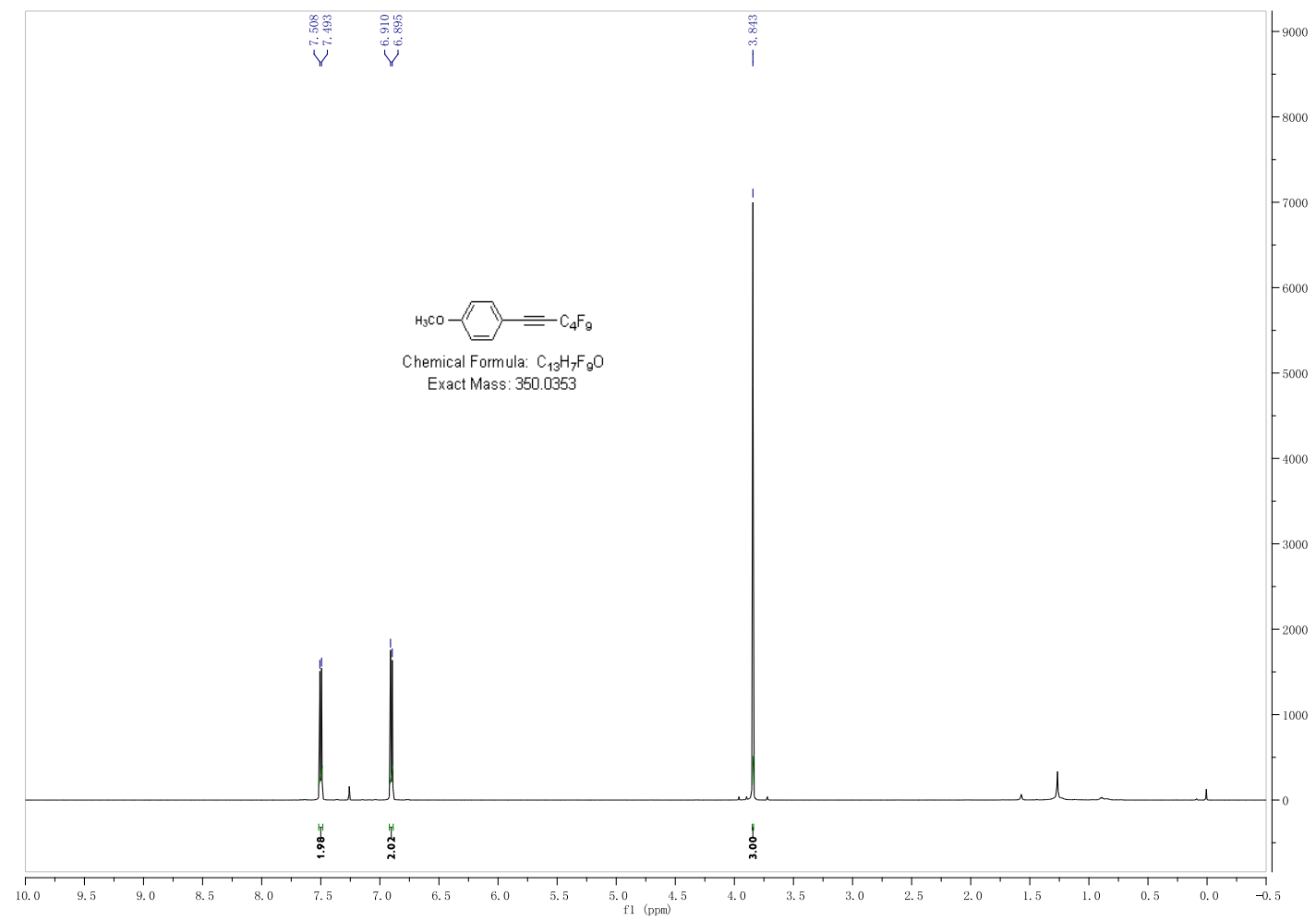


${ }^{19}$ F NMR $\left(564 \mathrm{MHz}, \mathrm{CDCl}_{3}\right)$

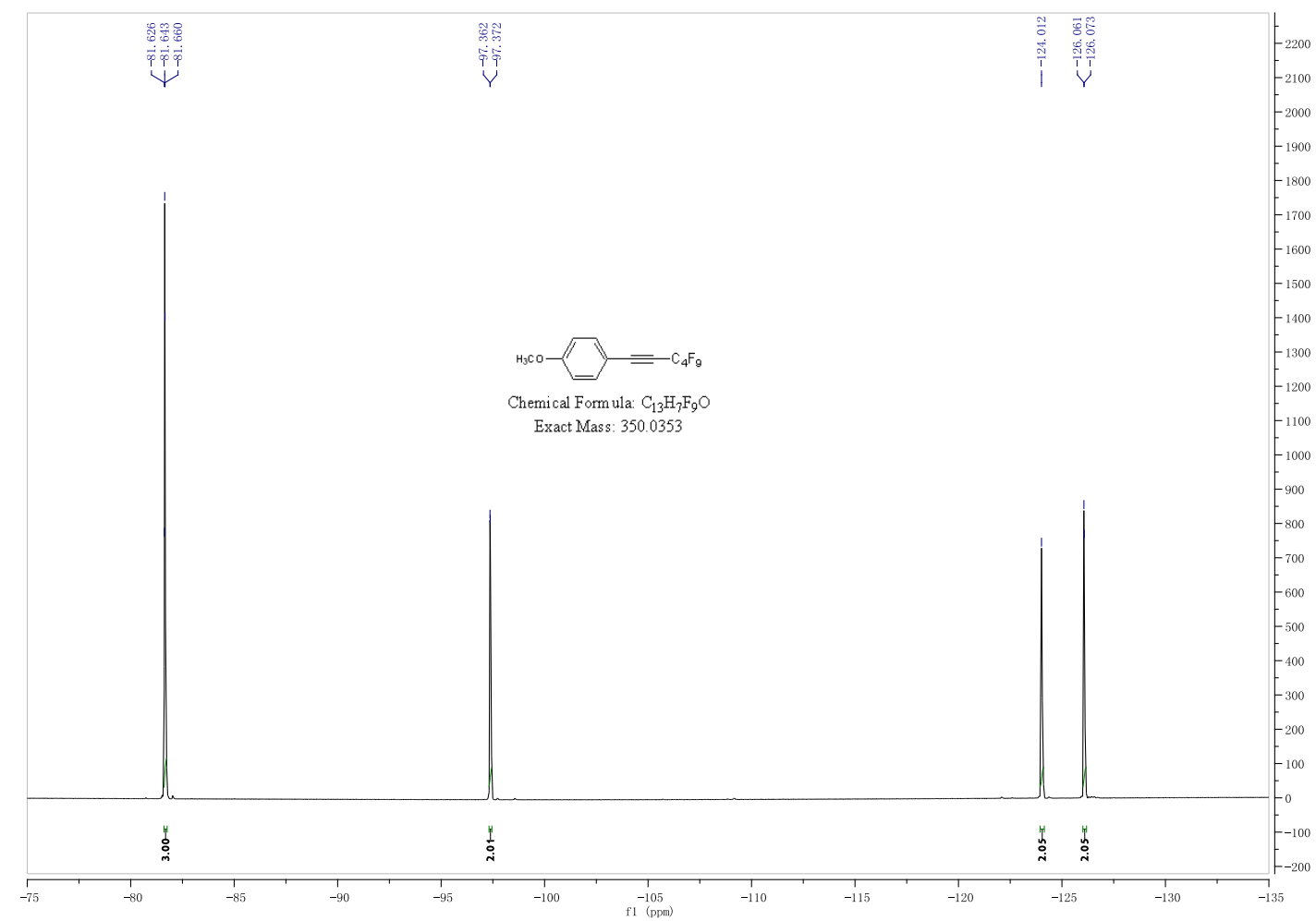

1,3-dimethoxy-5-(perfluorohex-1-yn-1-yl)benzene (6e)

${ }^{1} \mathbf{H}$ NMR $\left(600 \mathrm{MHz}, \mathrm{CDCl}_{3}\right)$

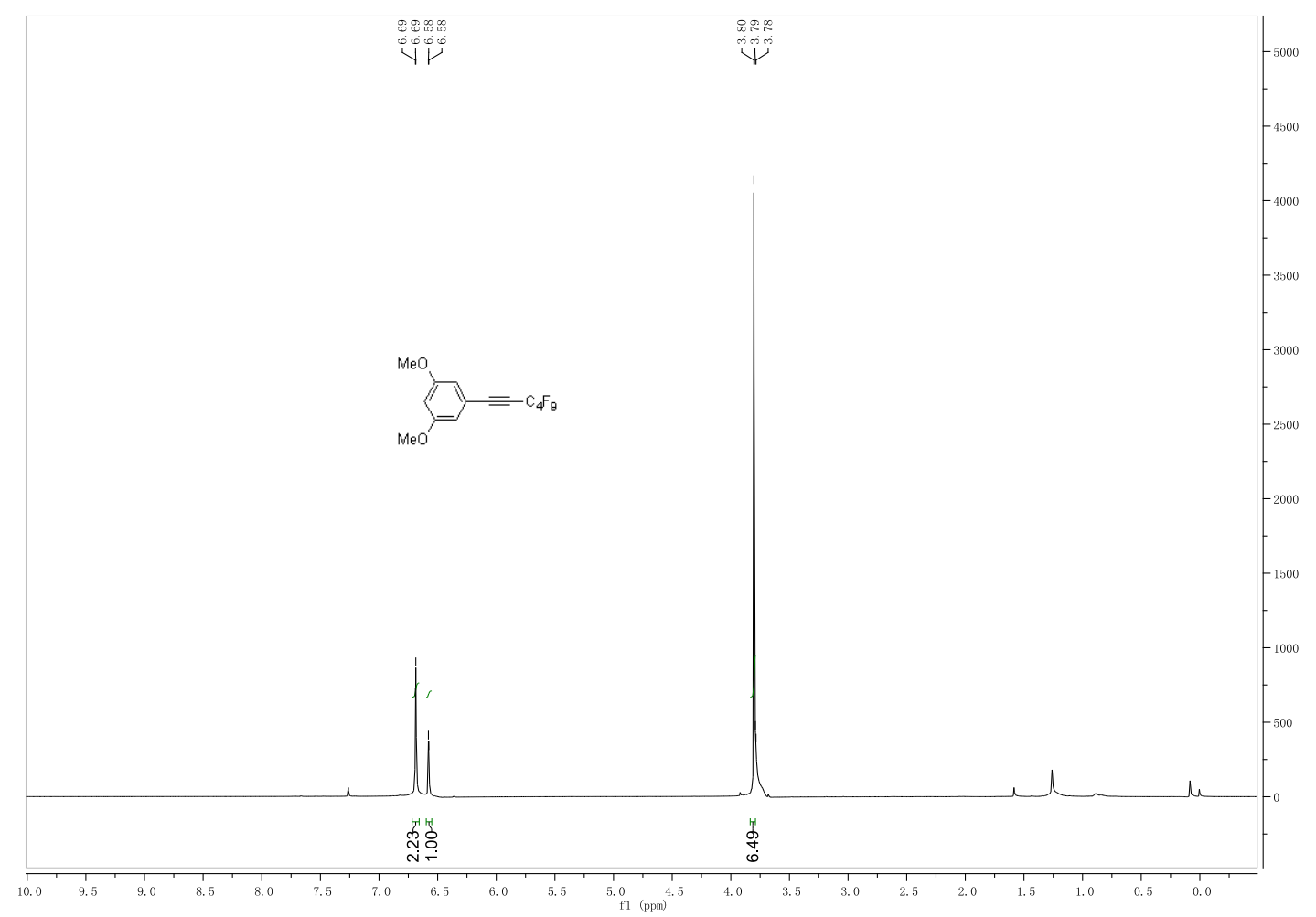


${ }^{19}$ F NMR (564 MHz, $\left.\mathrm{CDCl}_{3}\right)$

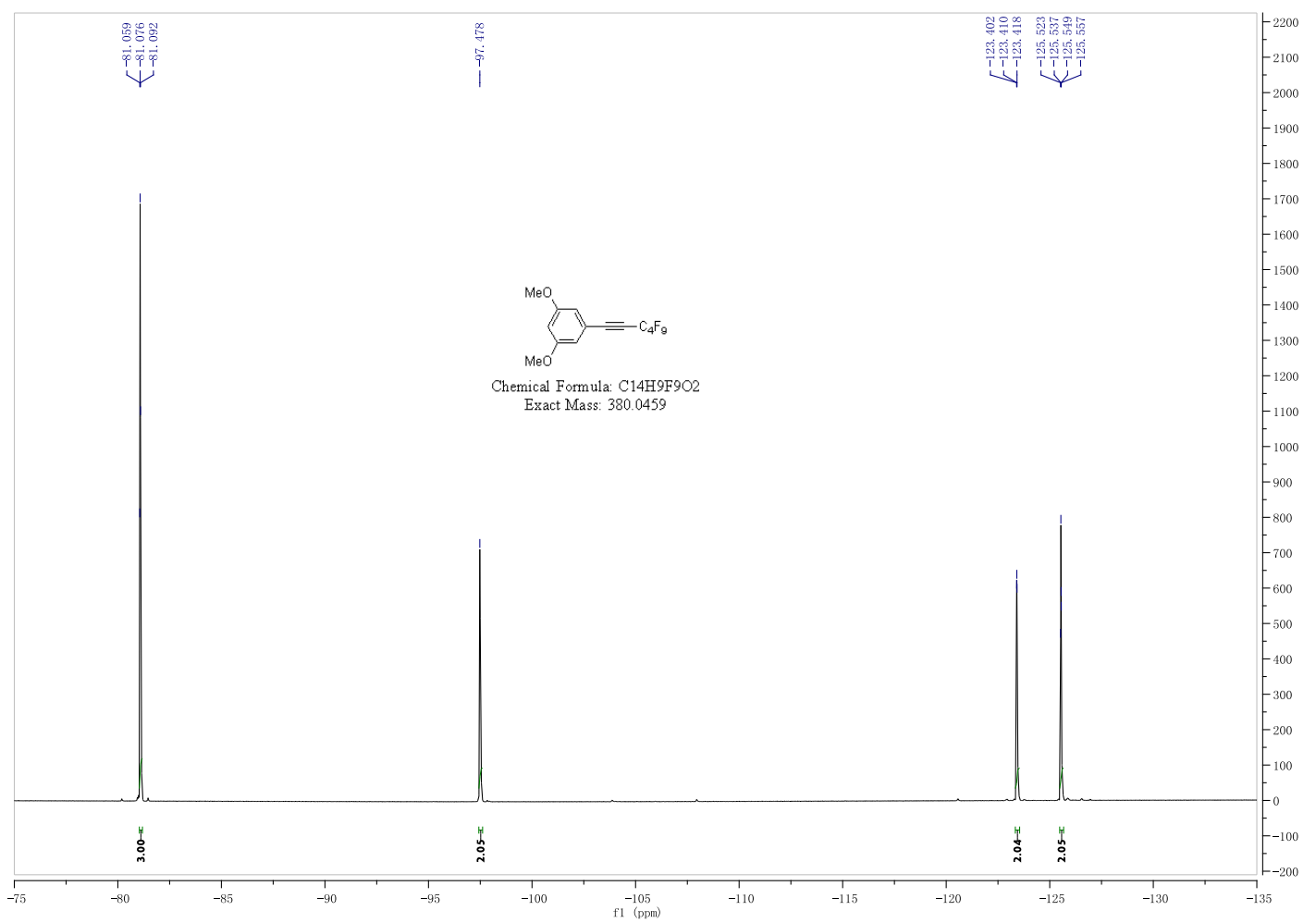

4-(perfluorohex-1-yn-1-yl)-1,1'-biphenyl (6f)

${ }^{1} \mathbf{H}$ NMR $\left(600 \mathrm{MHz}, \mathrm{CDCl}_{3}\right)$

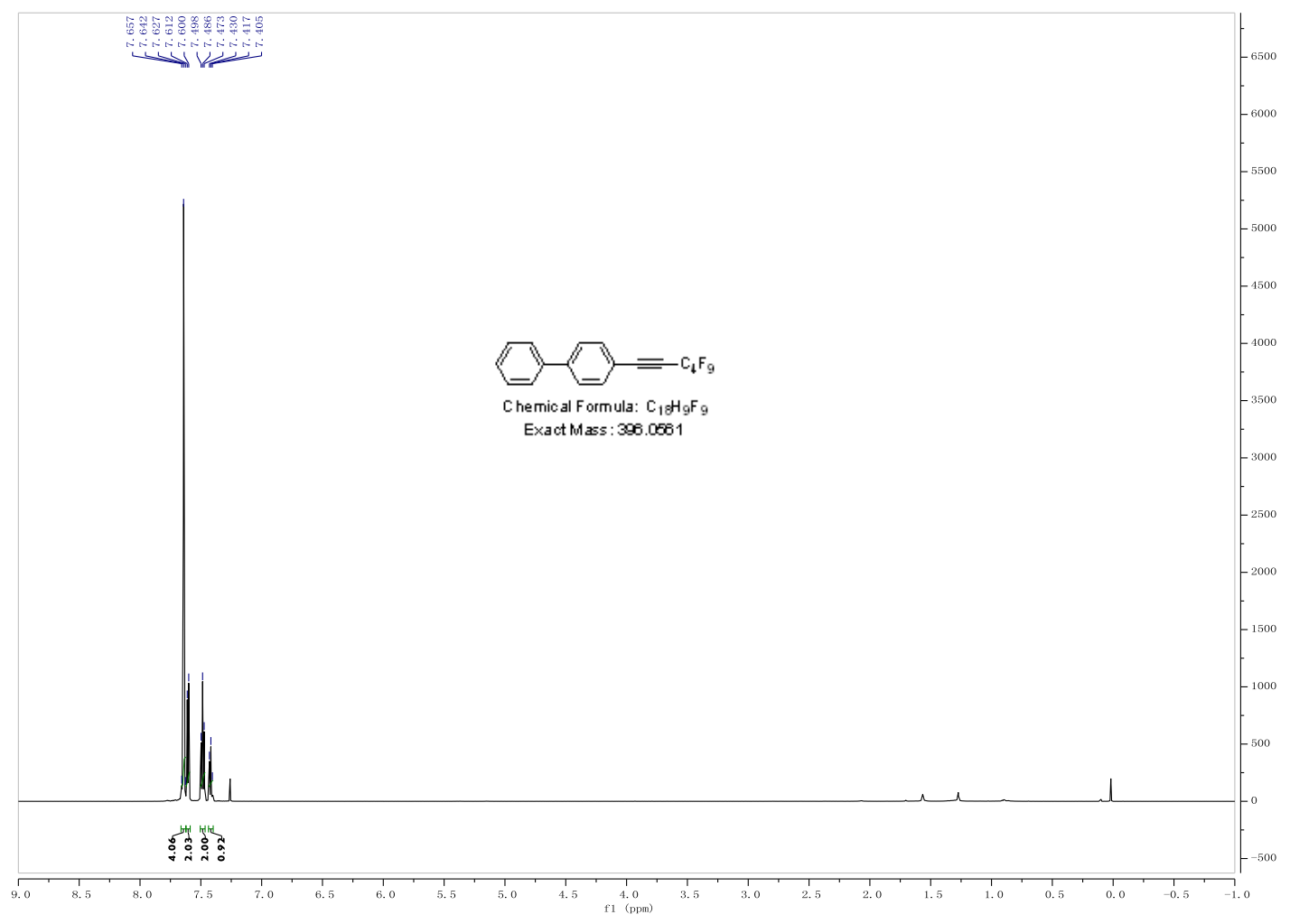


${ }^{19}$ F NMR $\left(564 \mathrm{MHz}, \mathrm{CDCl}_{3}\right)$

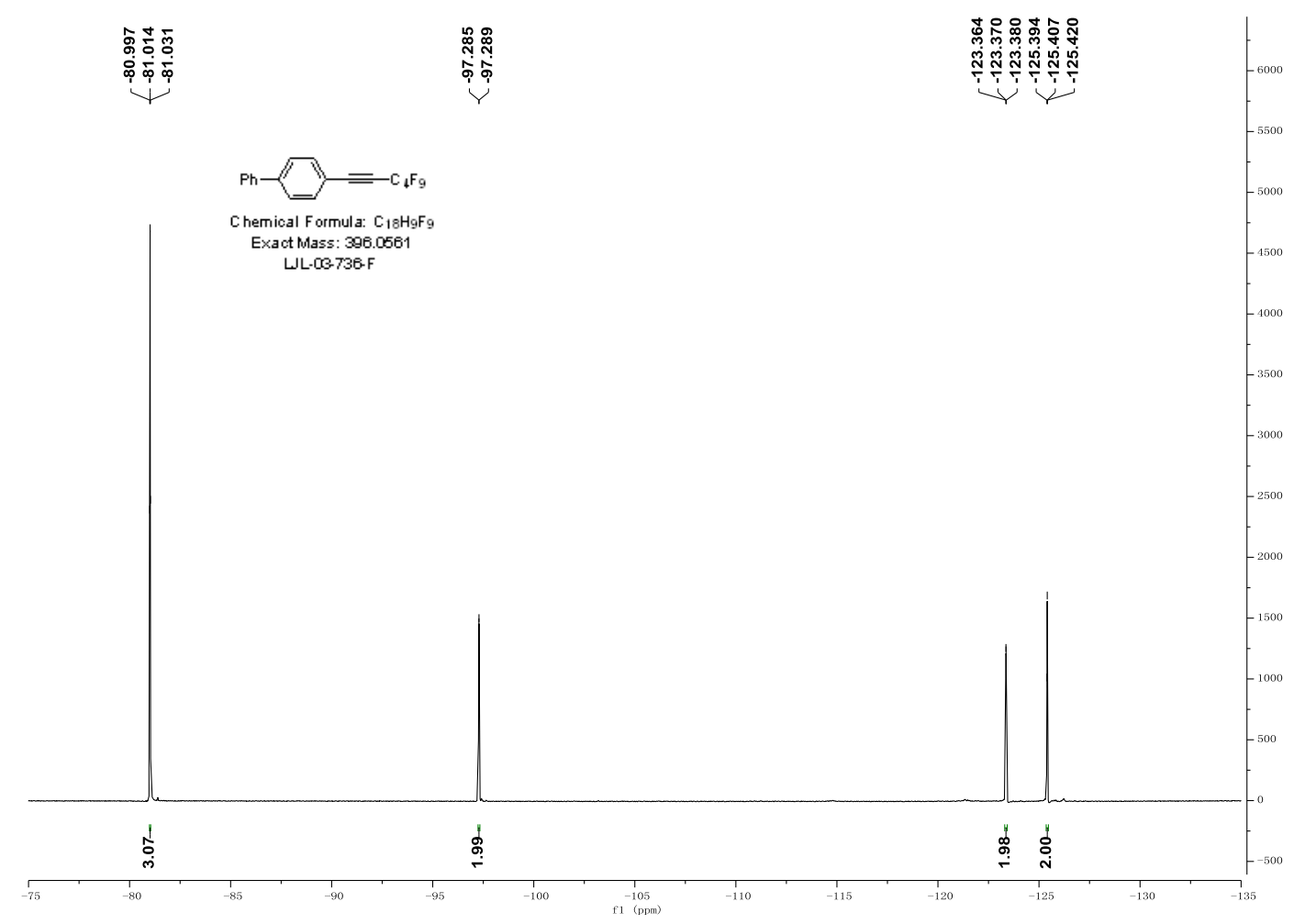

Methyl 4-(perfluorohex-1-yn-1-yl)benzoate (6g)

${ }^{1} \mathbf{H}$ NMR $\left(600 \mathrm{MHz}, \mathrm{CDCl}_{3}\right)$

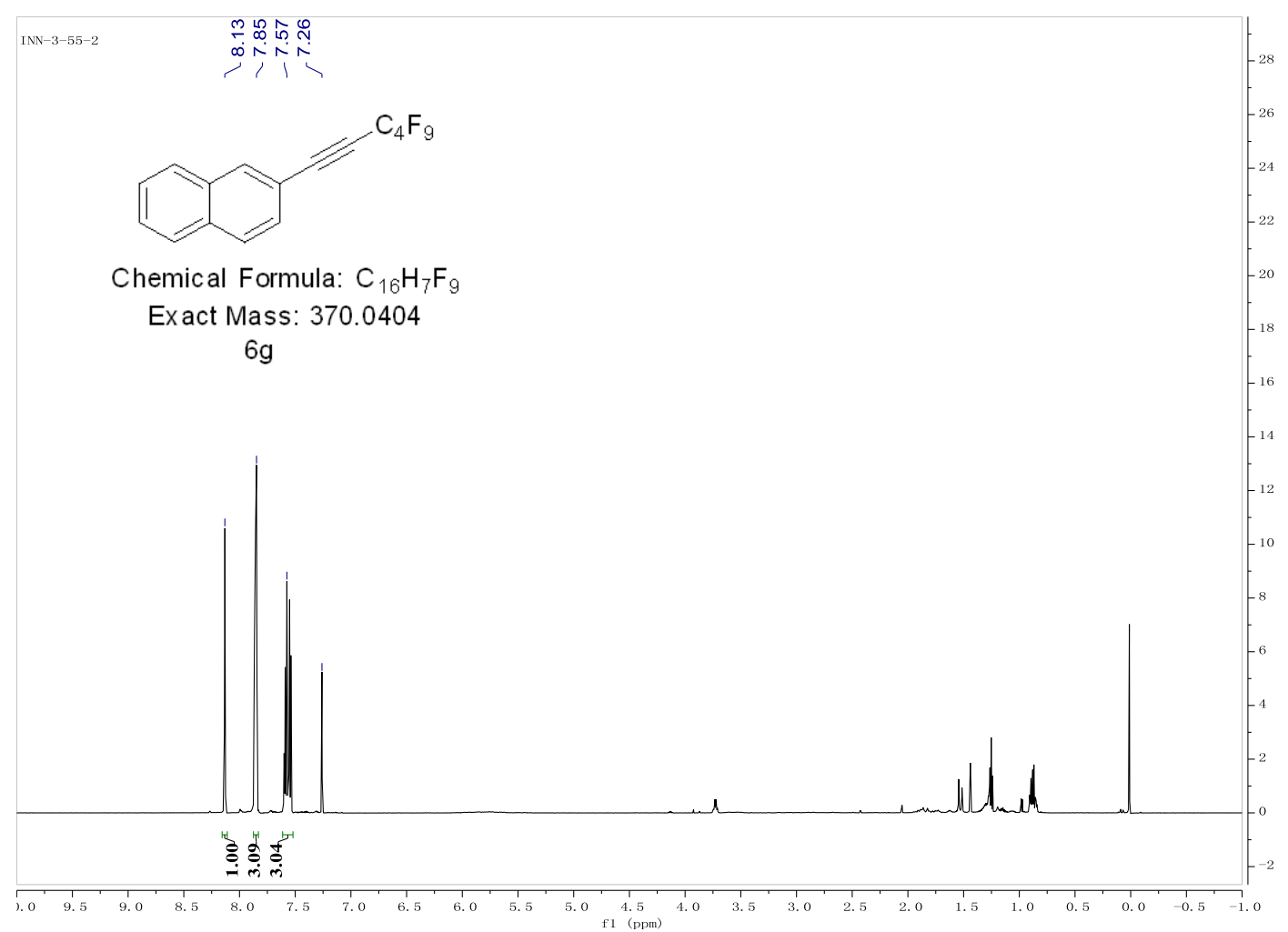


${ }^{19}$ F NMR $\left(564 \mathrm{MHz}, \mathrm{CDCl}_{3}\right)$

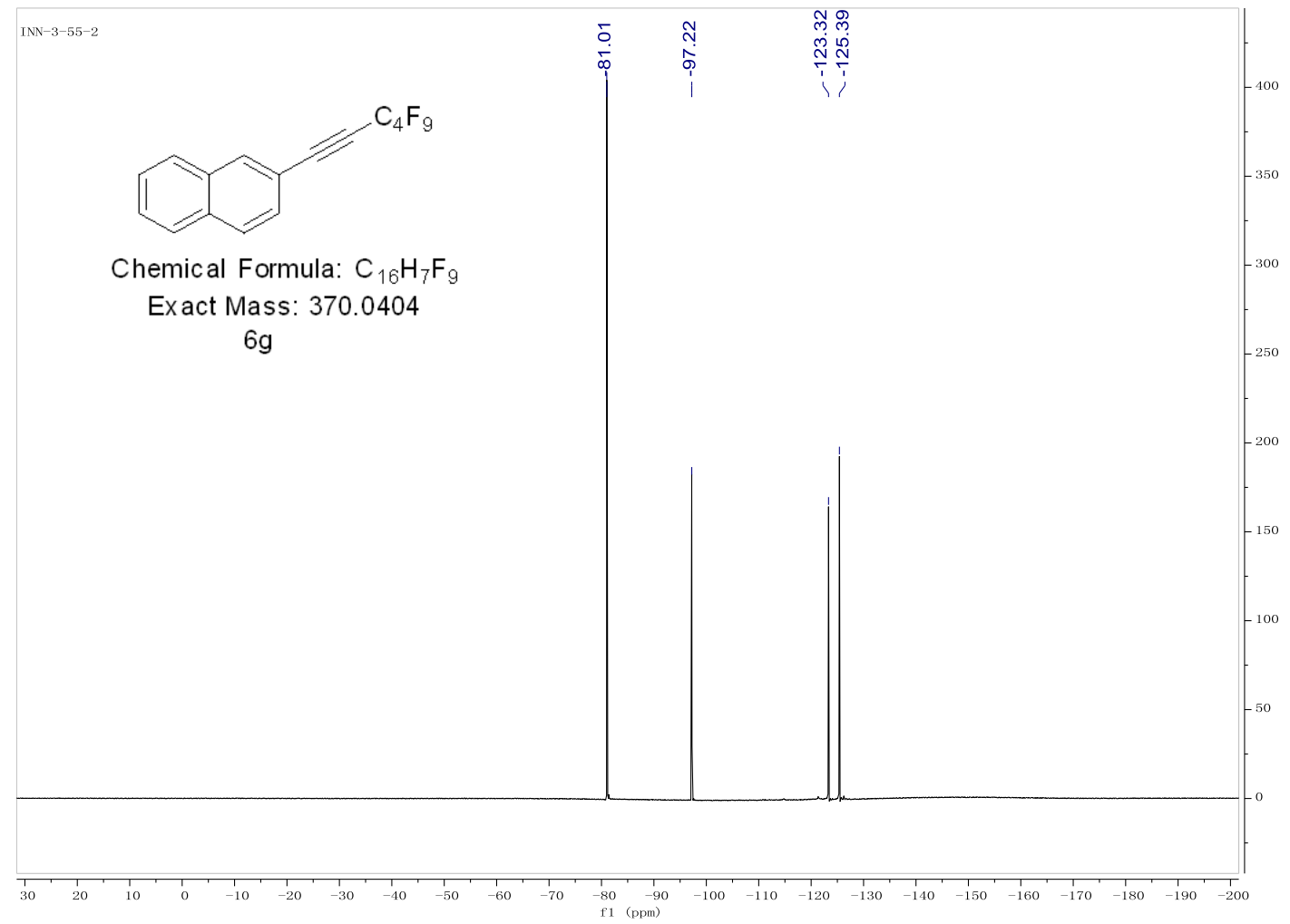

1-chloro-4-(perfluorohex-1-yn-1-yl)benzene (6h)

${ }^{1} \mathbf{H}$ NMR (600 MHz, $\left.\mathrm{CDCl}_{3}\right)$

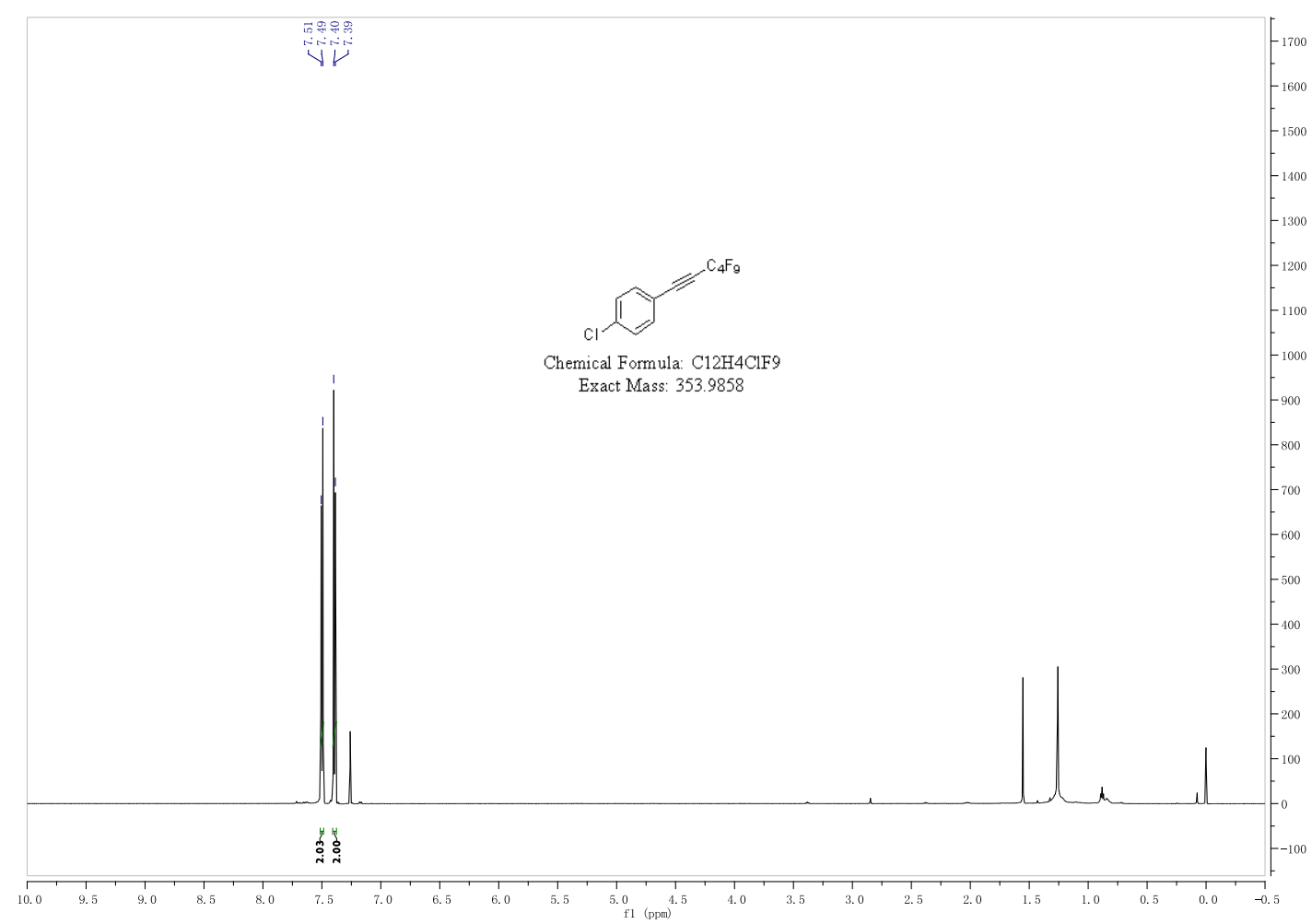


${ }^{19}$ F NMR $\left(564 \mathrm{MHz}, \mathrm{CDCl}_{3}\right)$

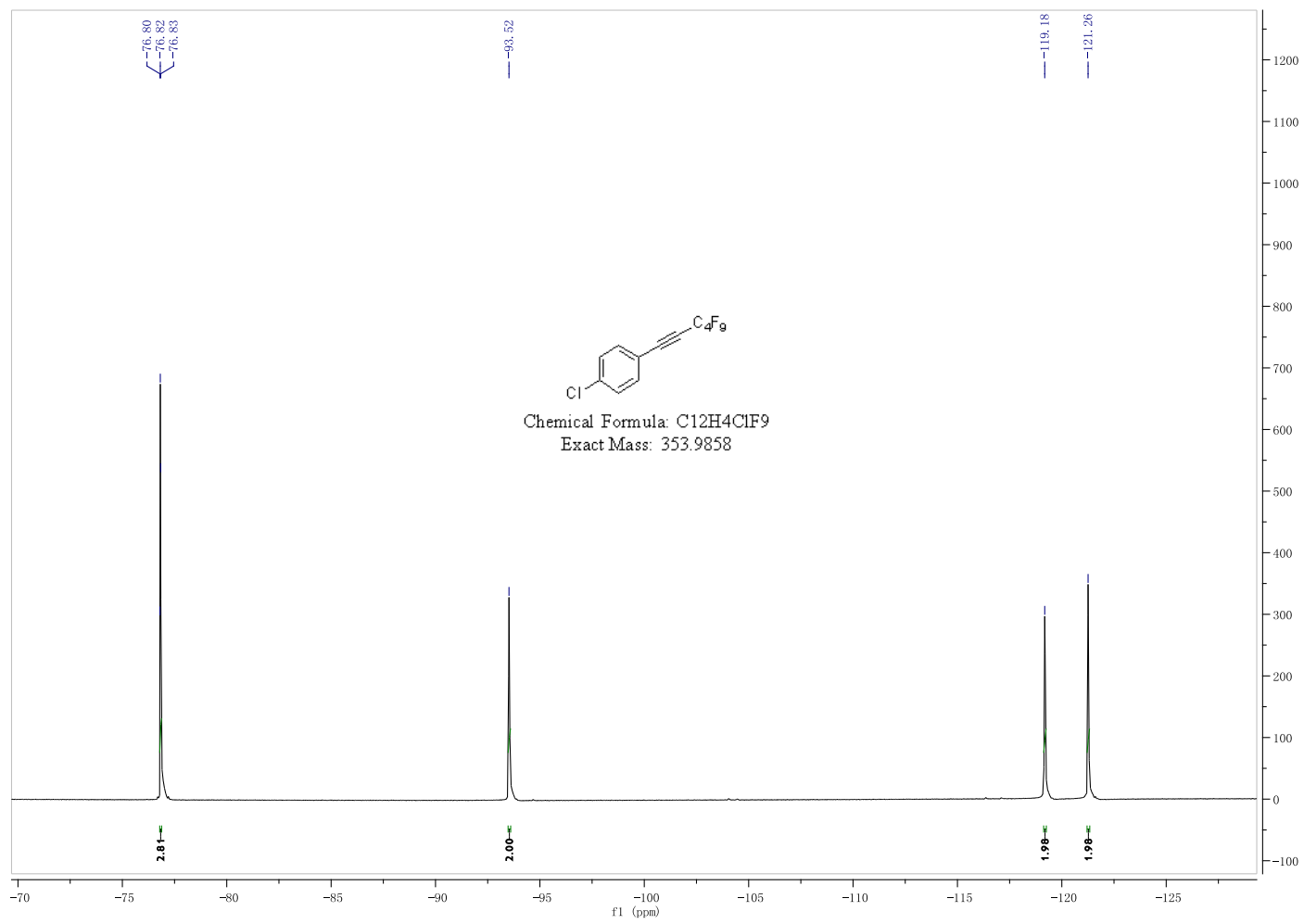

Methyl 4-(perfluorohex-1-yn-1-yl)benzoate (6i)

${ }^{1} \mathbf{H}$ NMR $\left(600 \mathrm{MHz}, \mathrm{CDCl}_{3}\right)$
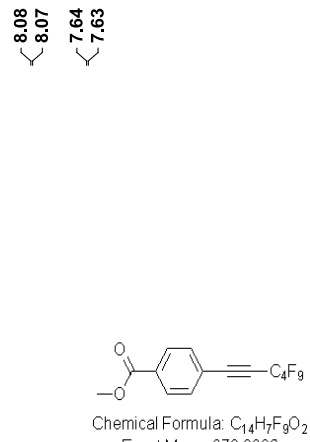

ExactMass: 3780302

LJL-03-831-H

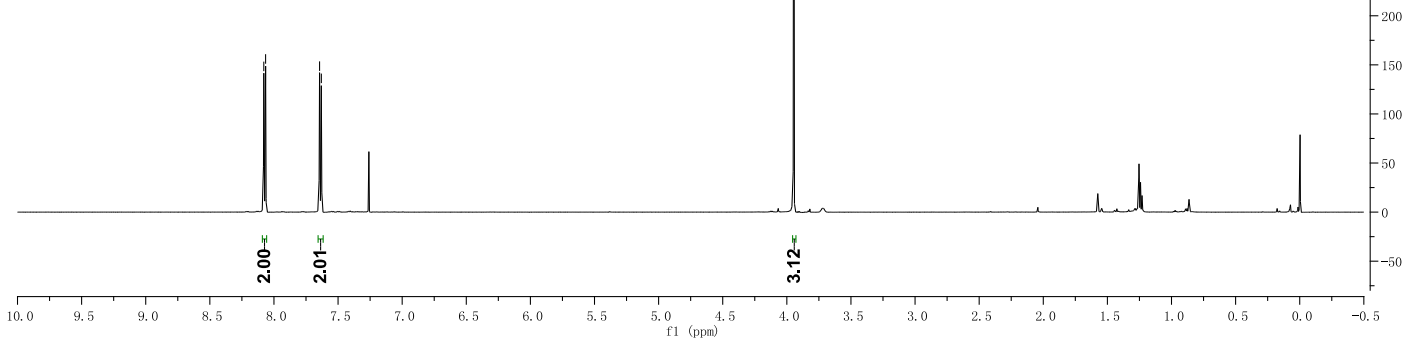


${ }^{19}$ F NMR $\left(564 \mathrm{MHz}, \mathrm{CDCl}_{3}\right)$

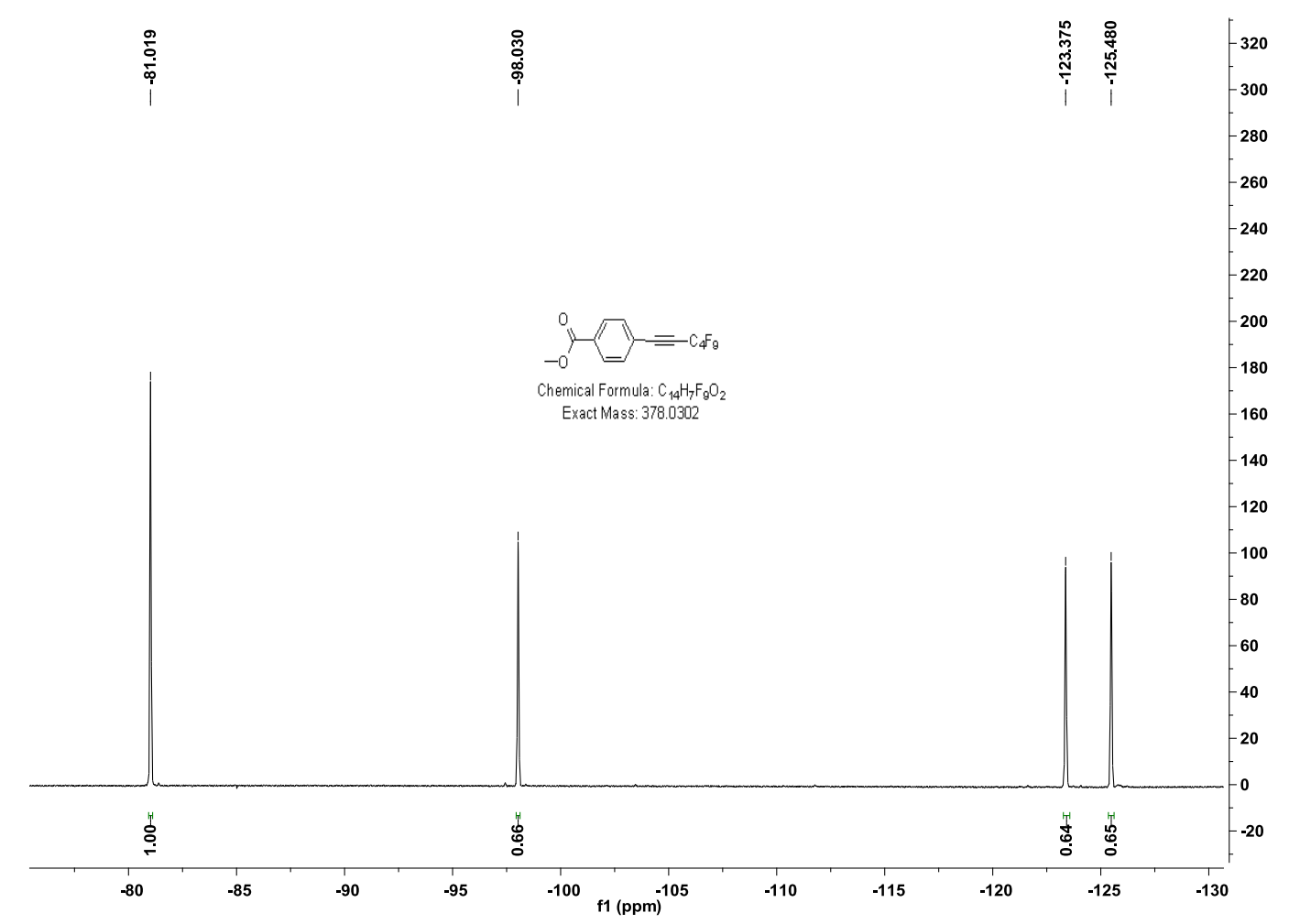

4-(Perfluorohex-1-yn-1-yl)thiophene(6j)

${ }^{1} \mathbf{H}$ NMR $\left(600 \mathrm{MHz}, \mathrm{CDCl}_{3}\right)$

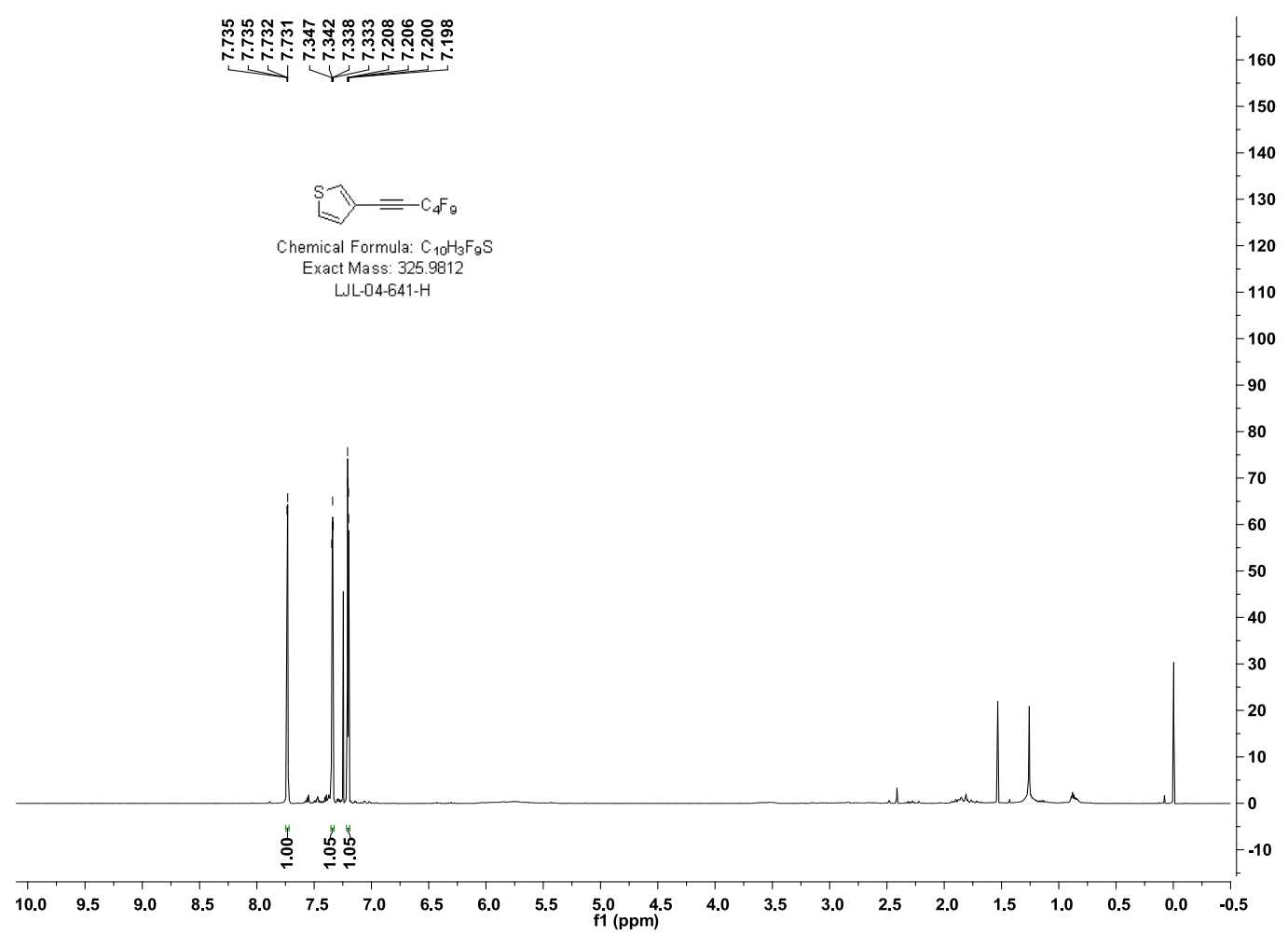


${ }^{19}$ F NMR $\left(564 \mathrm{MHz}, \mathrm{CDCl}_{3}\right)$

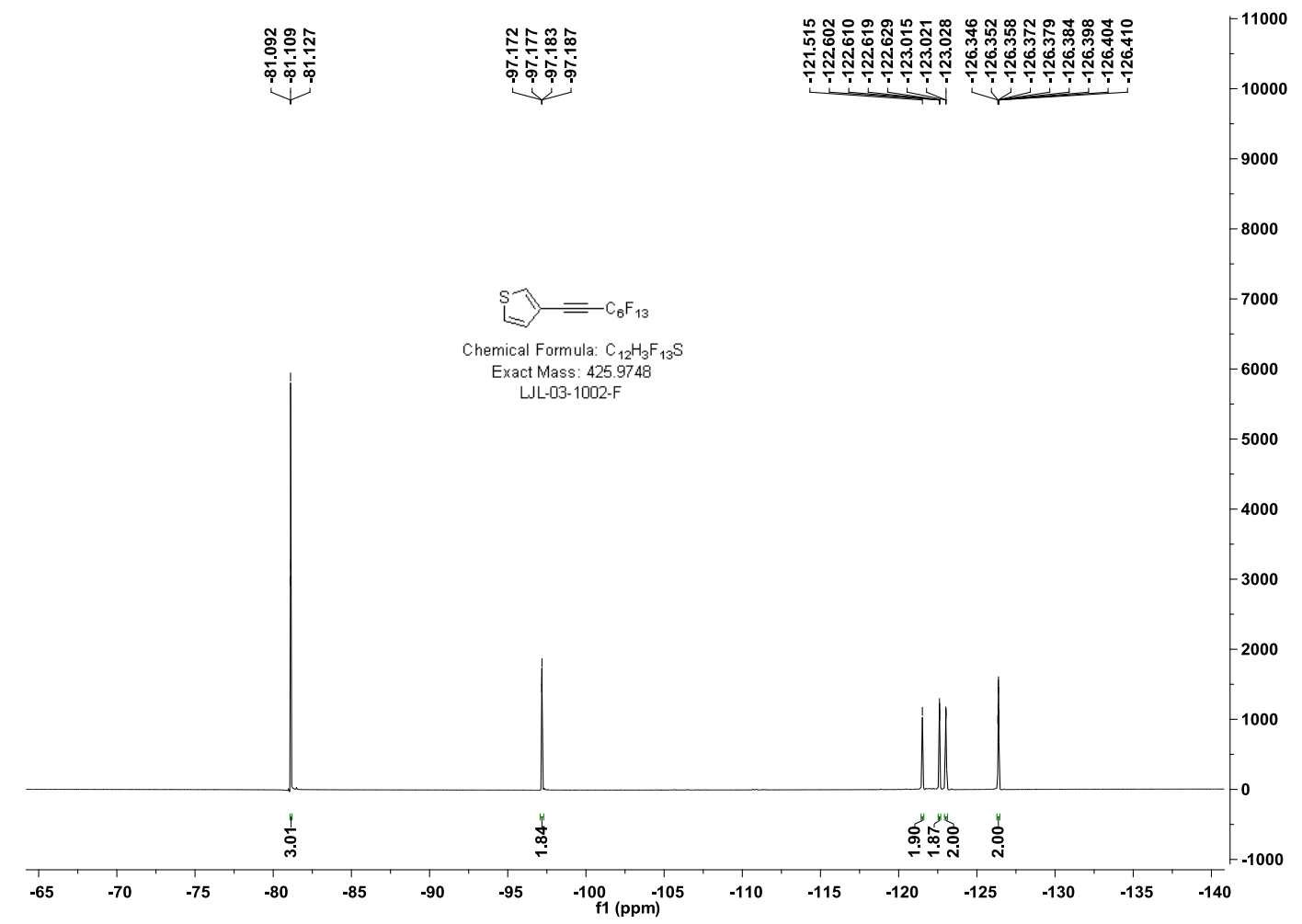

${ }^{13} \mathrm{C}$ NMR (150.8 MHz, $\left.\mathrm{CDCl}_{3}\right)$
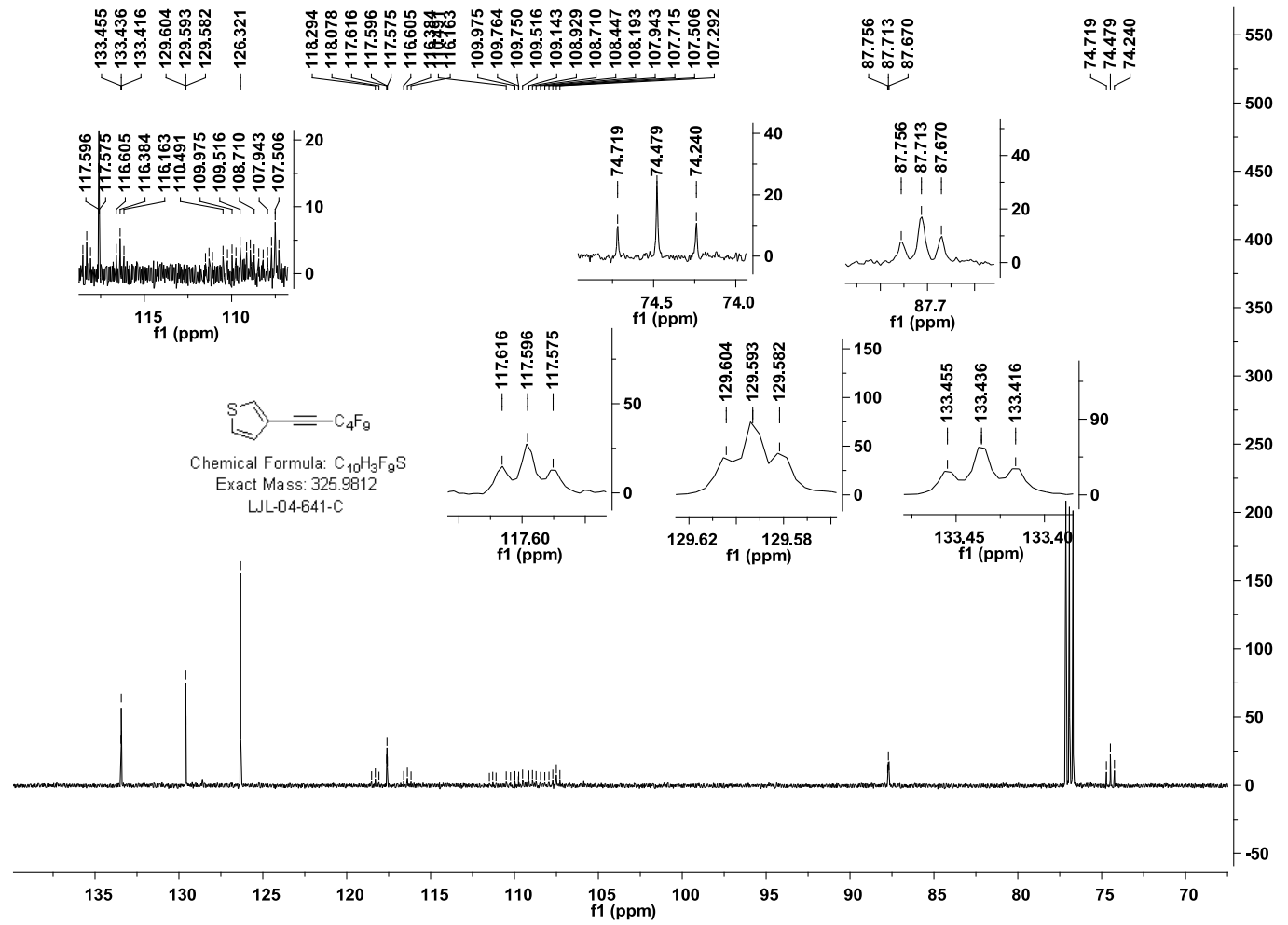
3-(Perfluorohex-1-yn-1-yl)pyridine(6k)

${ }^{1} \mathbf{H}$ NMR (600 MHz, $\left.\mathrm{CDCl}_{3}\right)$

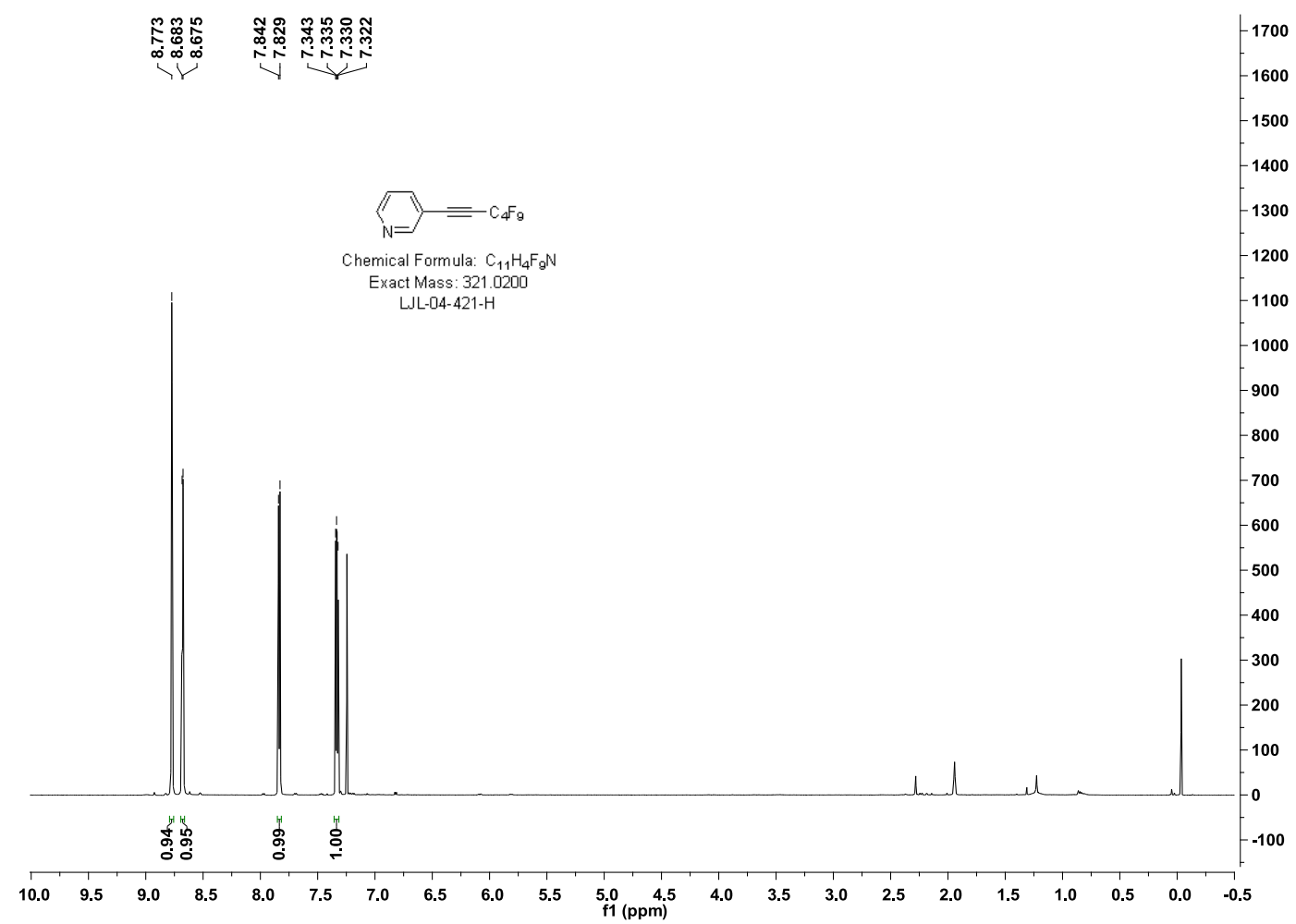

${ }^{19}$ F NMR $\left(564 \mathrm{MHz}, \mathrm{CDCl}_{3}\right)$

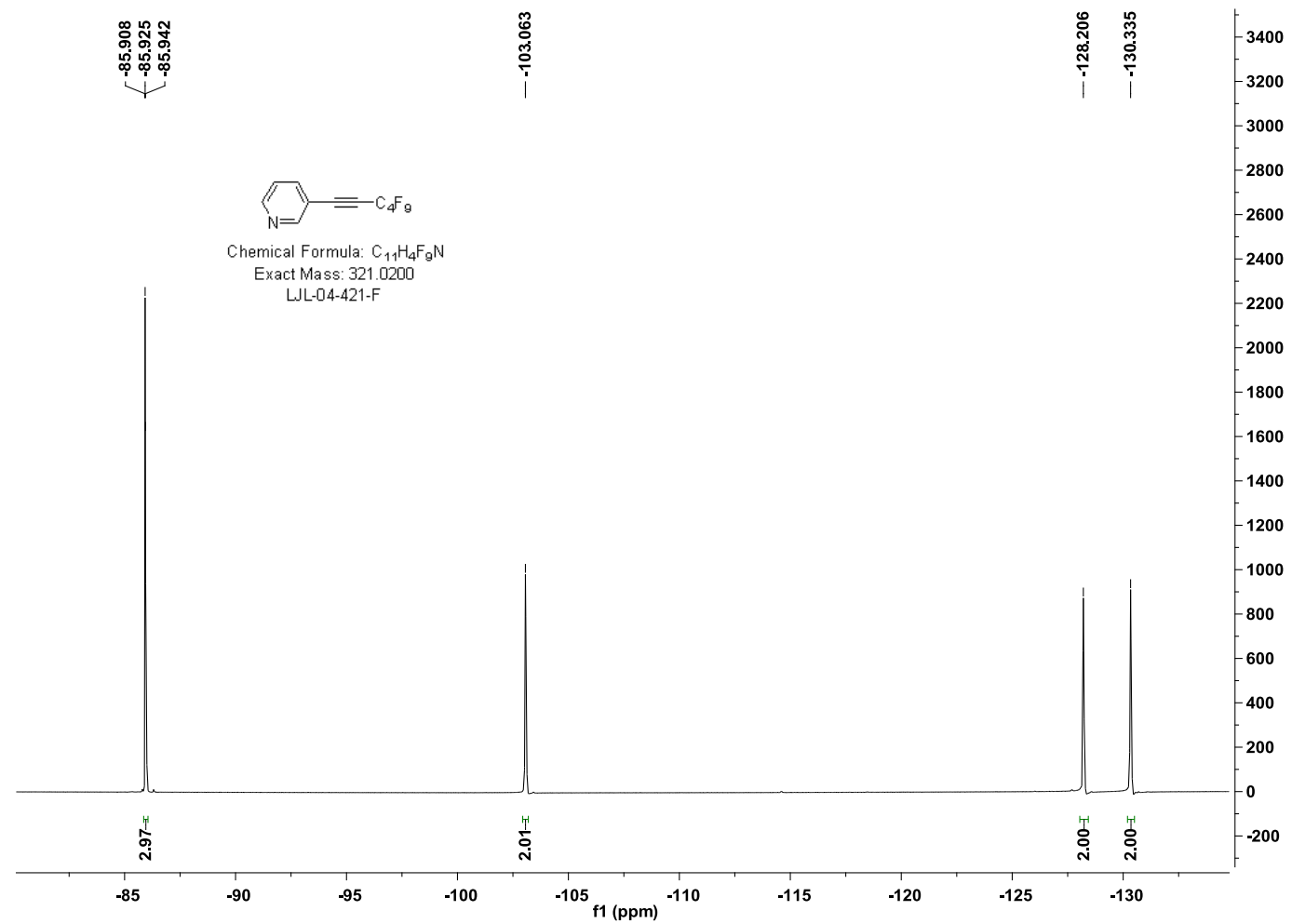


${ }^{13} \mathbf{C ~ N M R}\left(150.8 \mathrm{MHz}, \mathrm{CDCl}_{3}\right)$

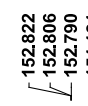
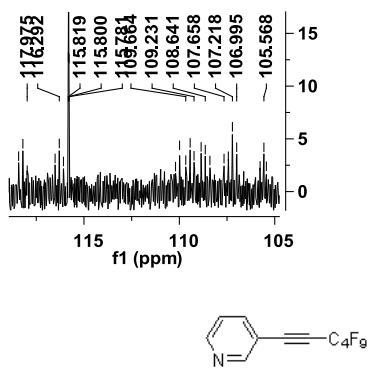

Chemical Formula: $\mathrm{C}_{11} \mathrm{H}_{4} \mathrm{~F}_{0}$

Exact Mass: 321.0200

LJL-04-421-C

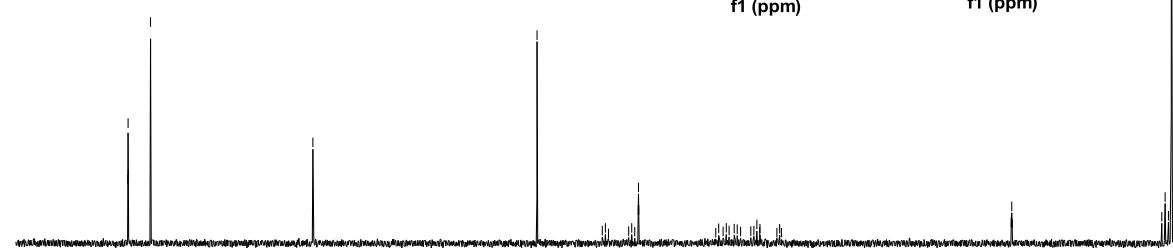

360

$-340$

$-320$

$\begin{array}{lllllllllllllllllll}160 & 155 & 150 & 145 & 140 & 135 & 130 & 125 & 120 & \begin{array}{l}115 \\ \mathrm{f} 1(\mathrm{ppm})\end{array} & 110 & 105 & 100 & 95 & 90 & 85 & 80 & 75 & 70\end{array}$

\section{1,1,1,2,2,3,3,4,4-Nonafluorohexadec-5-yne(61)}

${ }^{1} \mathbf{H}$ NMR $\left(600 \mathrm{MHz}, \mathrm{CDCl}_{3}\right)$

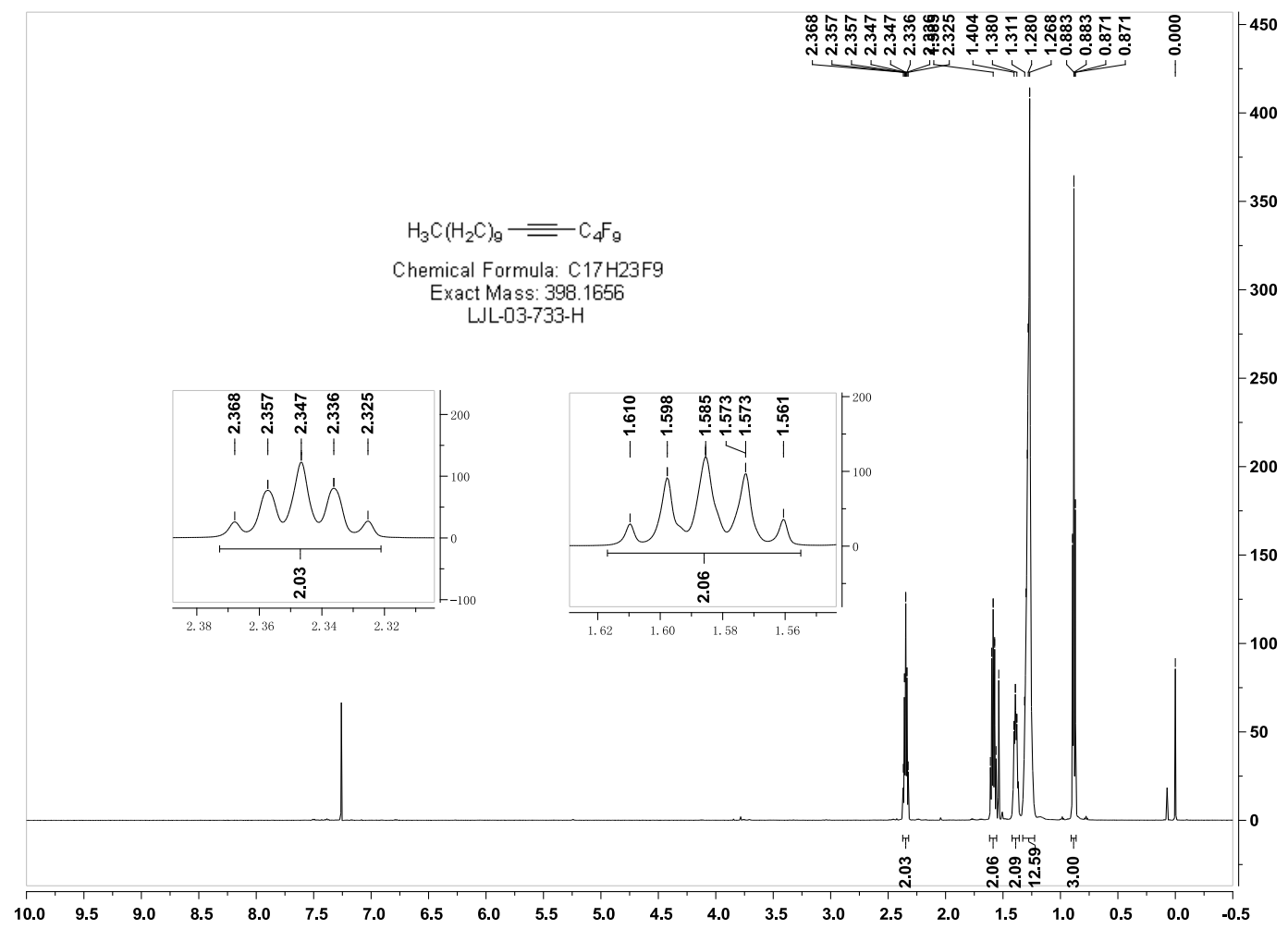


${ }^{19}$ F NMR $\left(564 \mathrm{MHz}, \mathrm{CDCl}_{3}\right)$

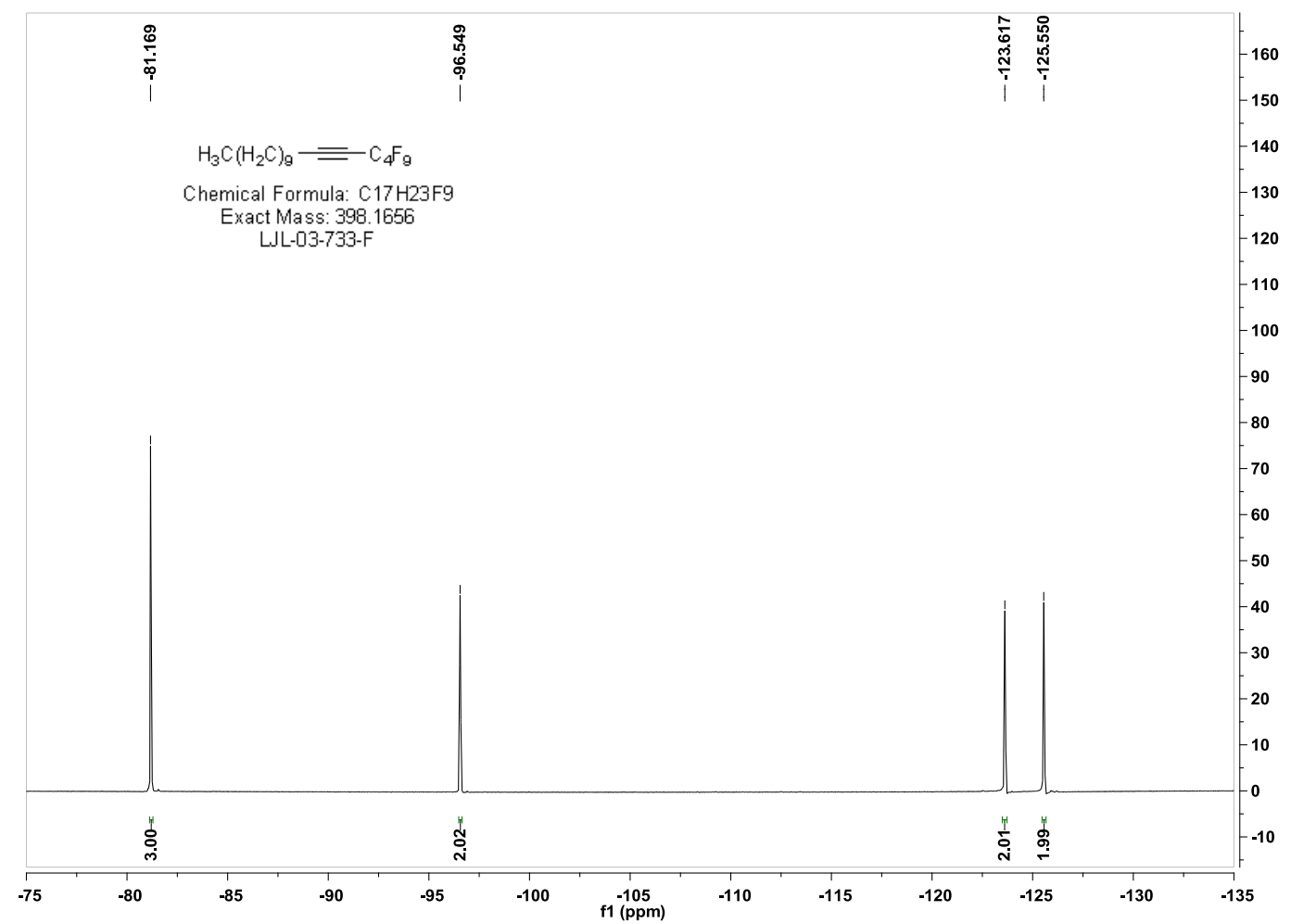

${ }^{13}$ C NMR (150.8 MHz, $\left.\mathrm{CDCl}_{3}\right)$

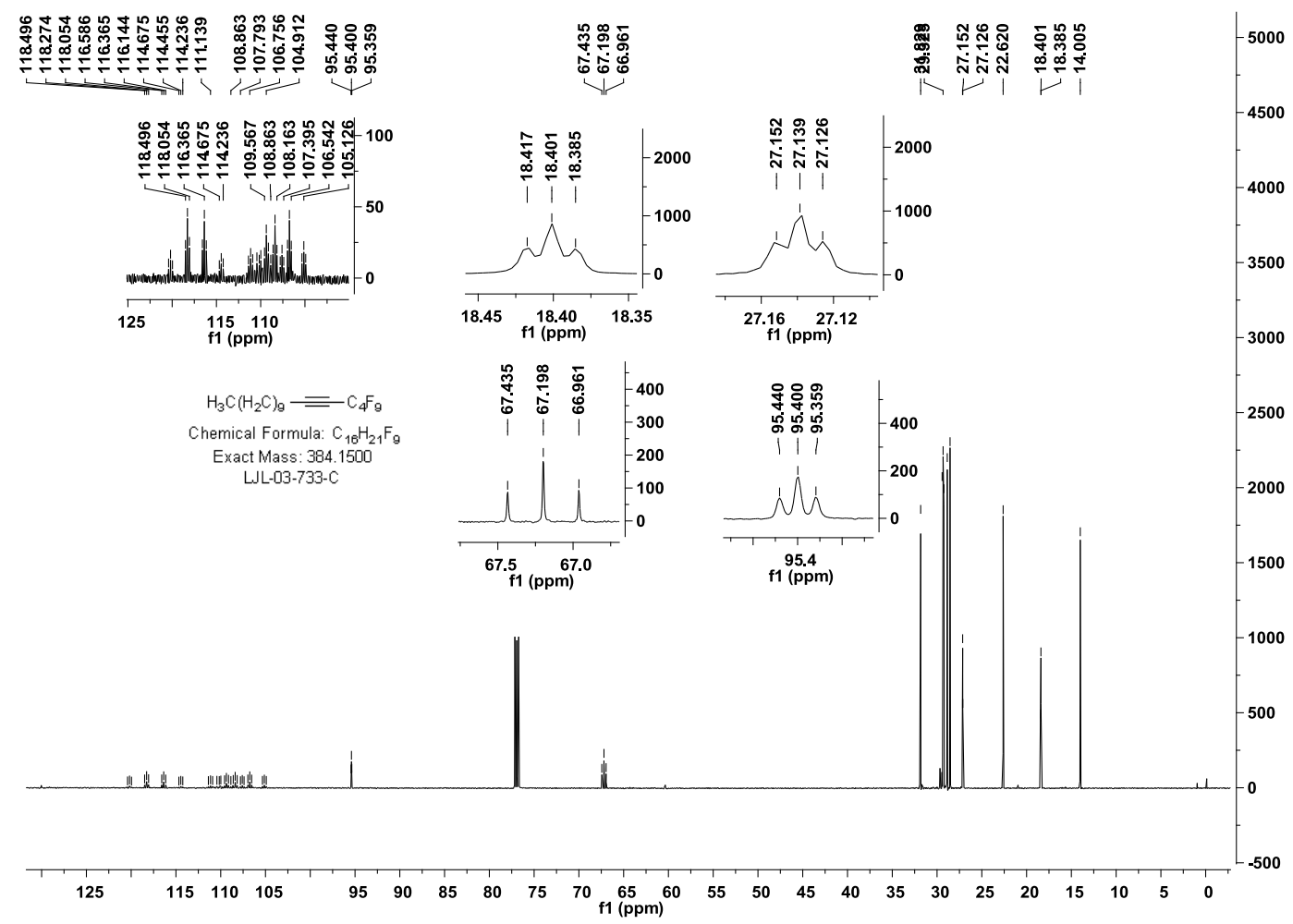


tert-Butyldimethyl((6,6,7,7,8,8,9,9,9-nonafluoronon-4-yn-1-yl)oxy)silane (6m)

${ }^{1} \mathbf{H}$ NMR (600 MHz, $\left.\mathrm{CDCl}_{3}\right)$

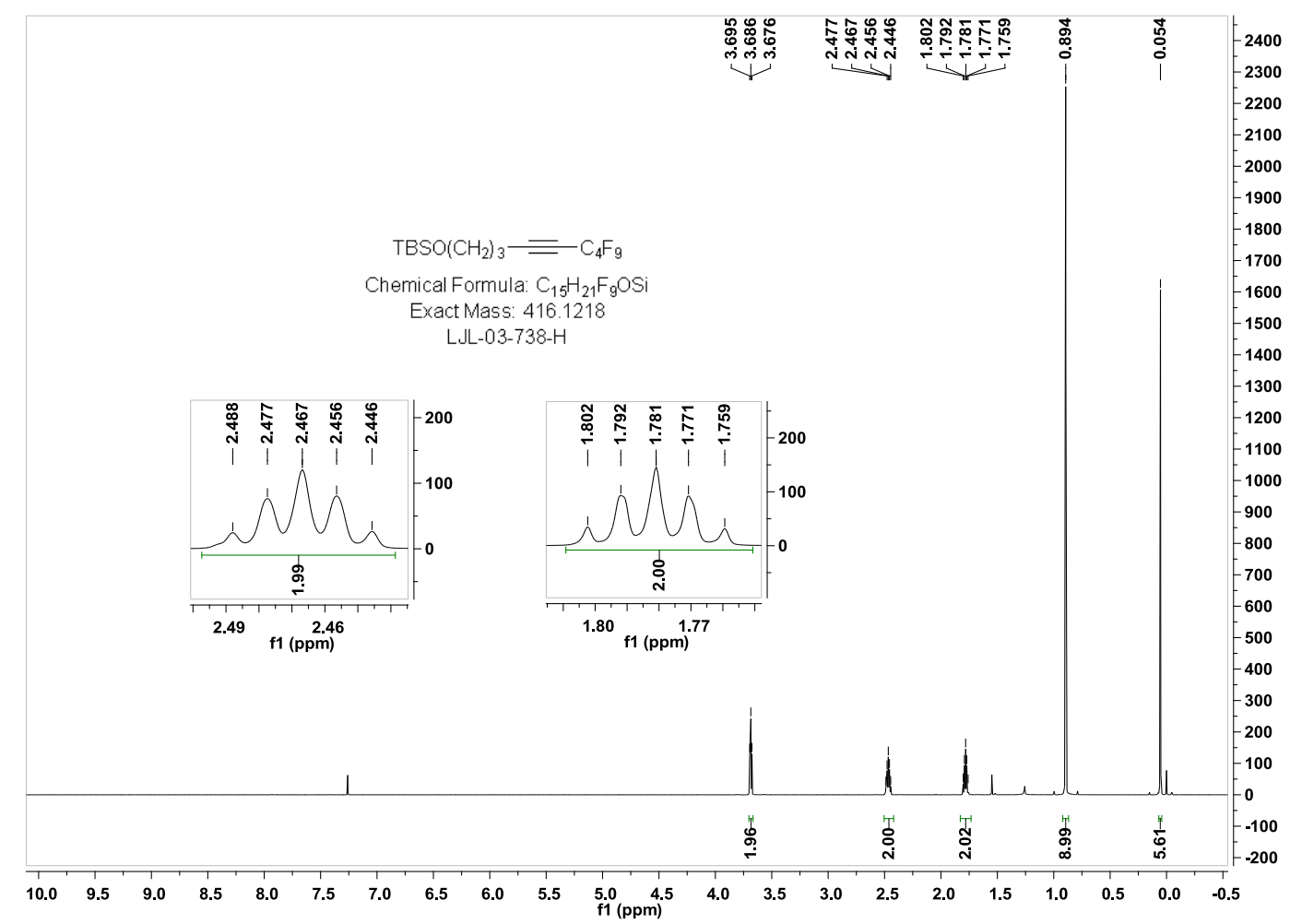

${ }^{19}$ F NMR (564 MHz, $\left.\mathrm{CDCl}_{3}\right)$

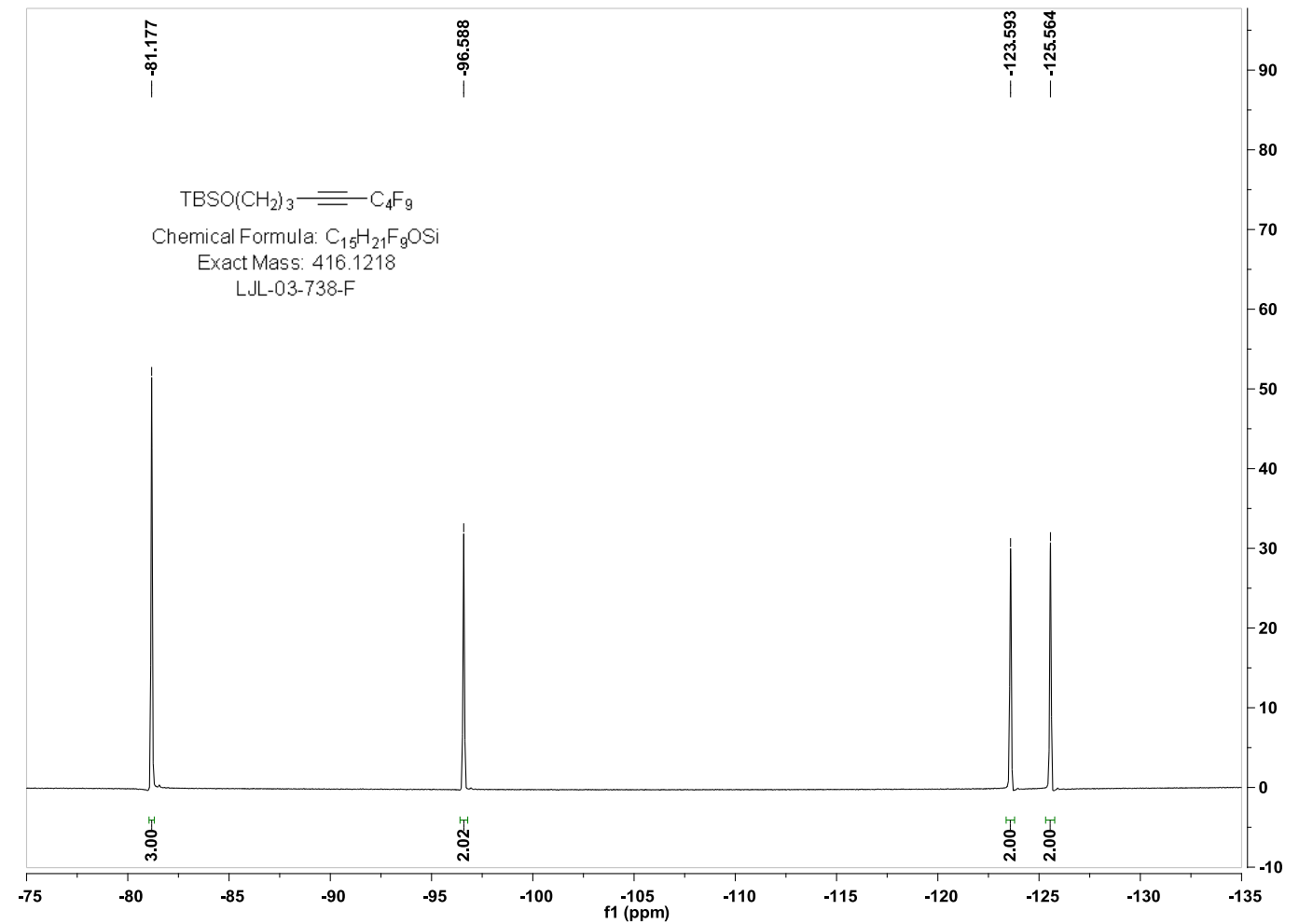


${ }^{13} \mathbf{C ~ N M R}\left(150.8 \mathrm{MHz}, \mathrm{CDCl}_{3}\right)$

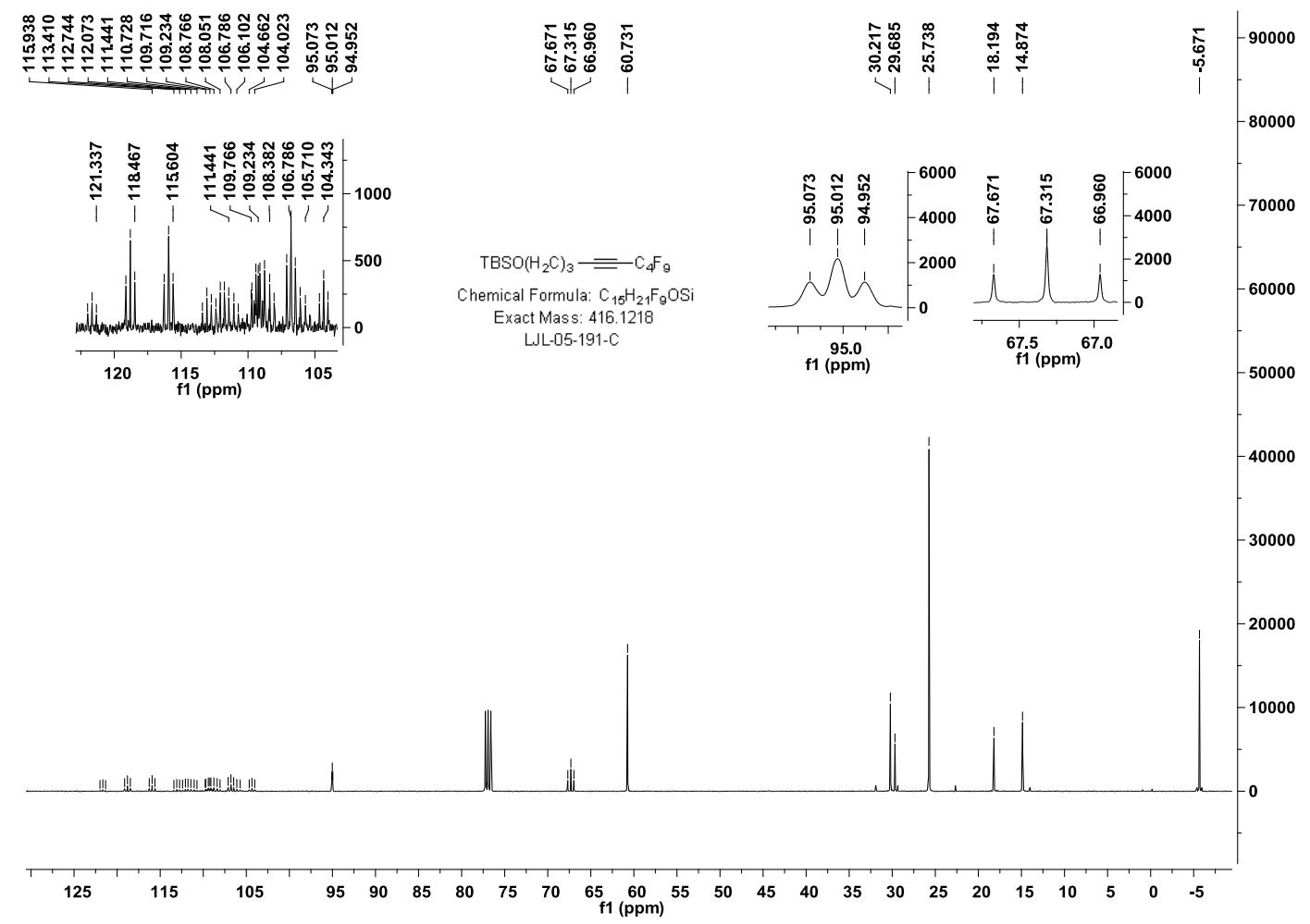

6,6,7,7,8,8,9,9,9-Nonafluoronon-4-yn-1-yl acetate (6n)

${ }^{1} \mathbf{H}$ NMR $\left(600 \mathrm{MHz}, \mathrm{CDCl}_{3}\right)$

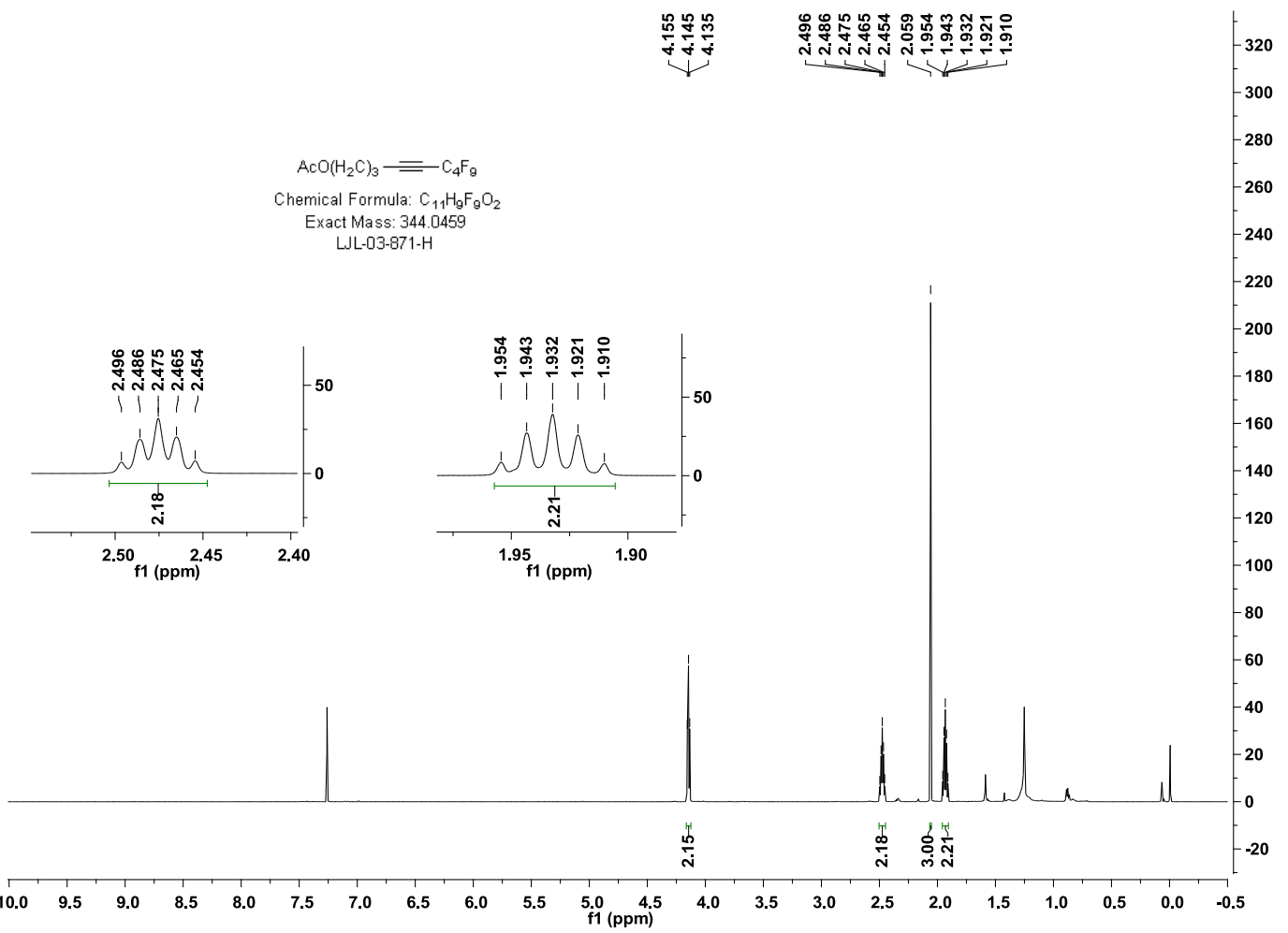


${ }^{19}$ F NMR $\left(564 \mathrm{MHz}, \mathrm{CDCl}_{3}\right)$

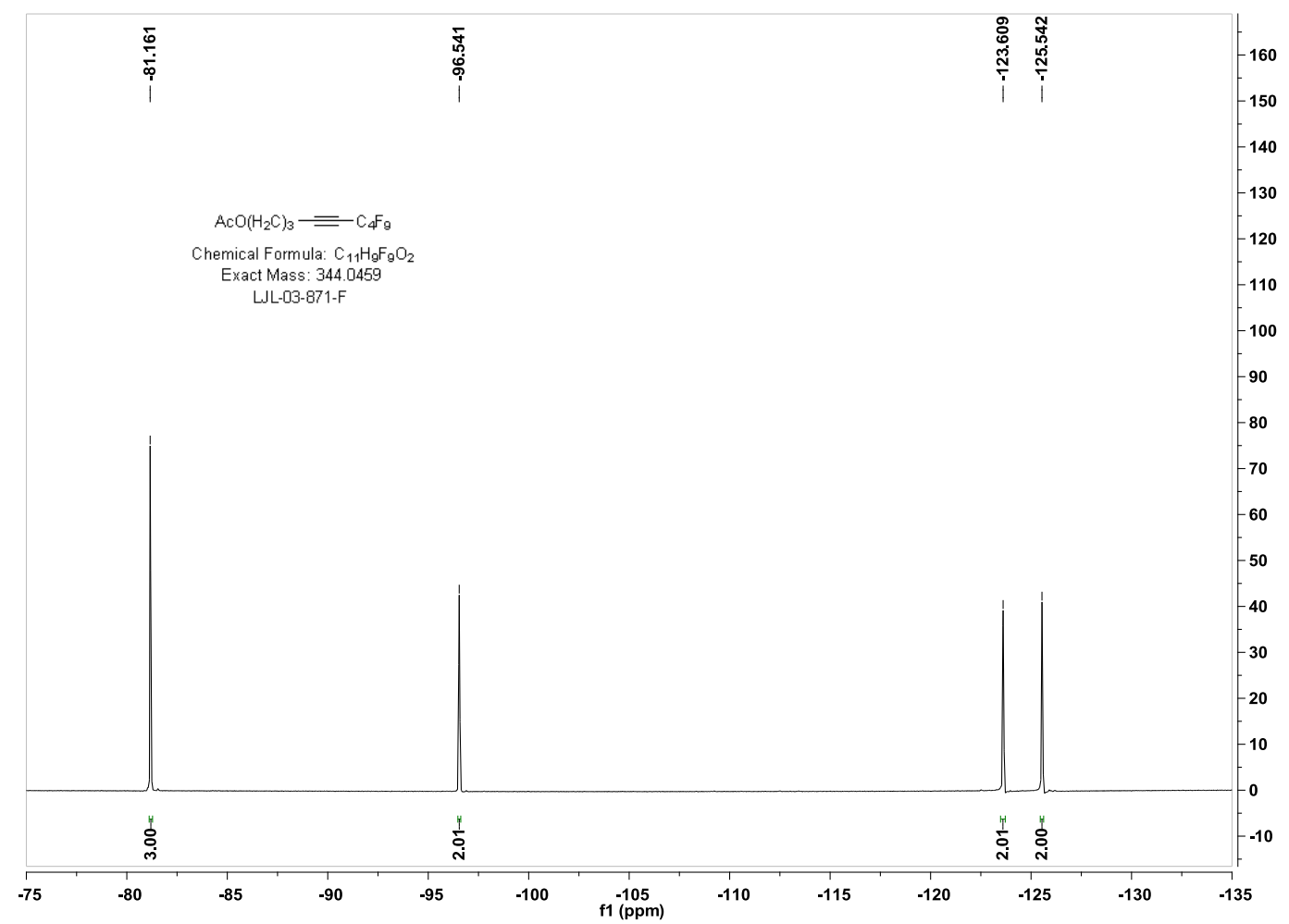

${ }^{13} \mathrm{C}$ NMR (150.8 MHz, $\left.\mathrm{CDCl}_{3}\right)$

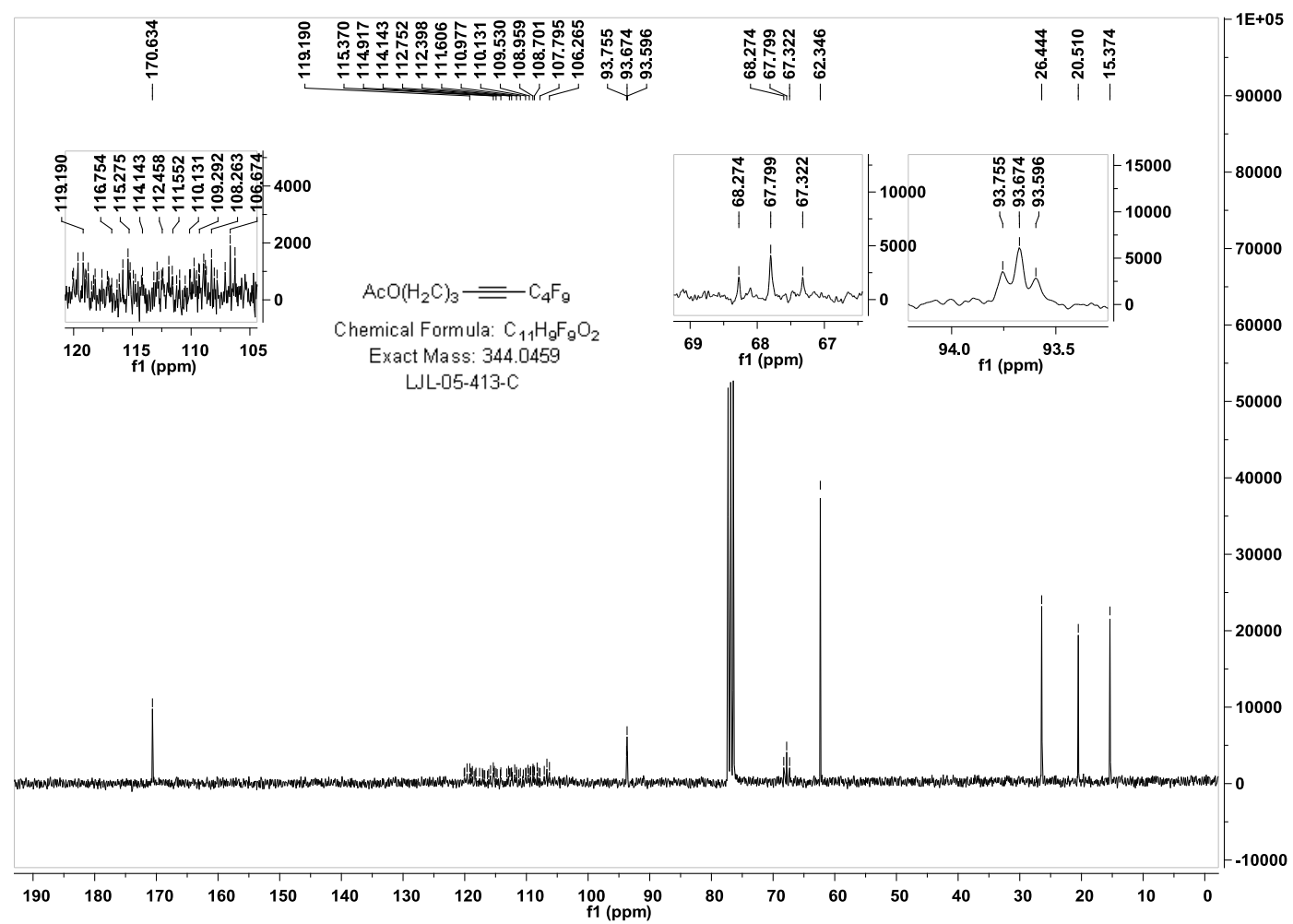


6,6,7,7,8,8,9,9,9-Nonafluoronon-4-yn-1-yl 4-cyanobenzoate (6o)

${ }^{1} \mathbf{H}$ NMR $\left(600 \mathrm{MHz}, \mathrm{CDCl}_{3}\right)$

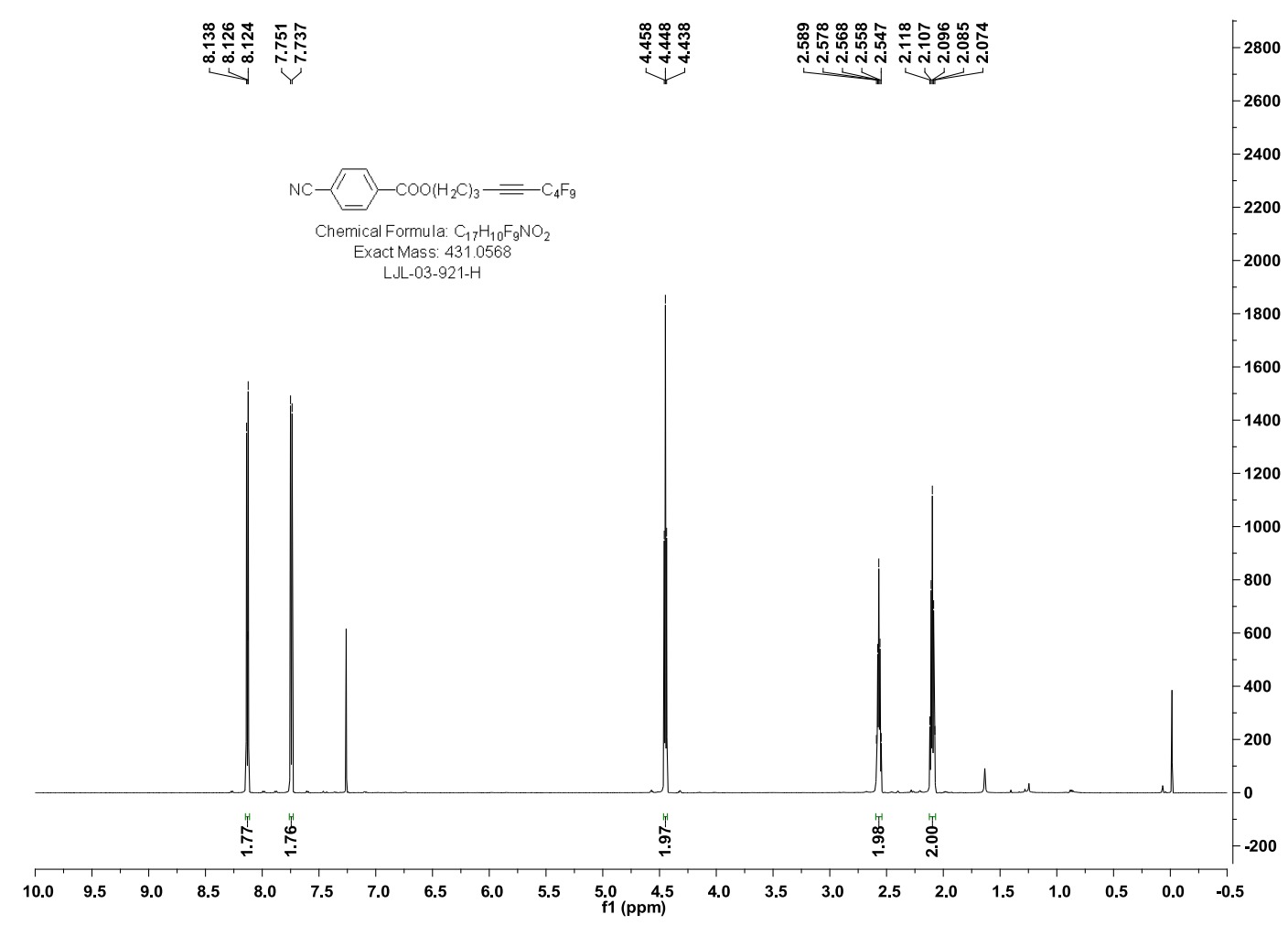

${ }^{19}$ F NMR $\left(564 \mathrm{MHz}, \mathrm{CDCl}_{3}\right)$

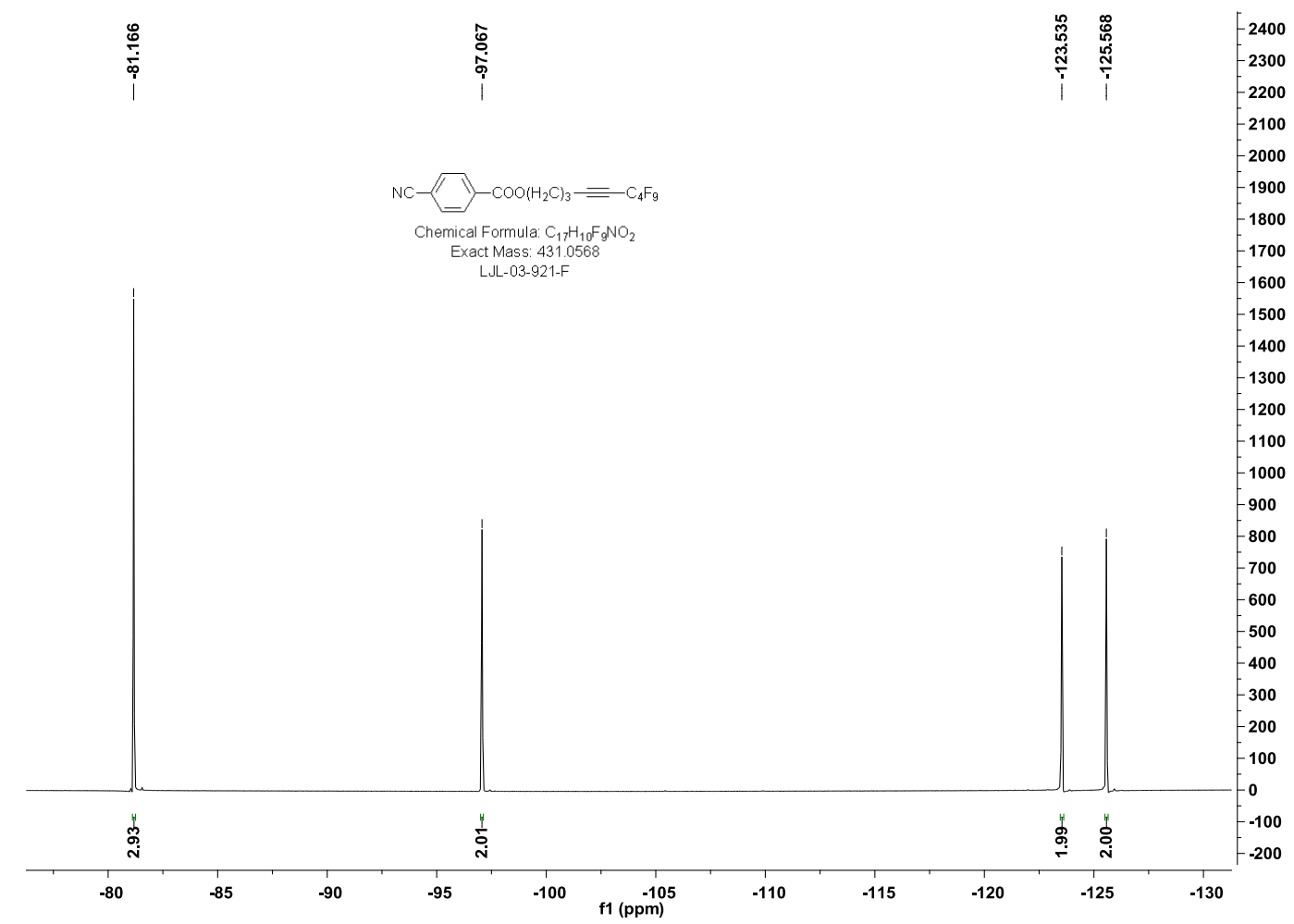


${ }^{13} \mathbf{C}$ NMR $\left(150.8 \mathrm{MHz}, \mathrm{CDCl}_{3}\right)$

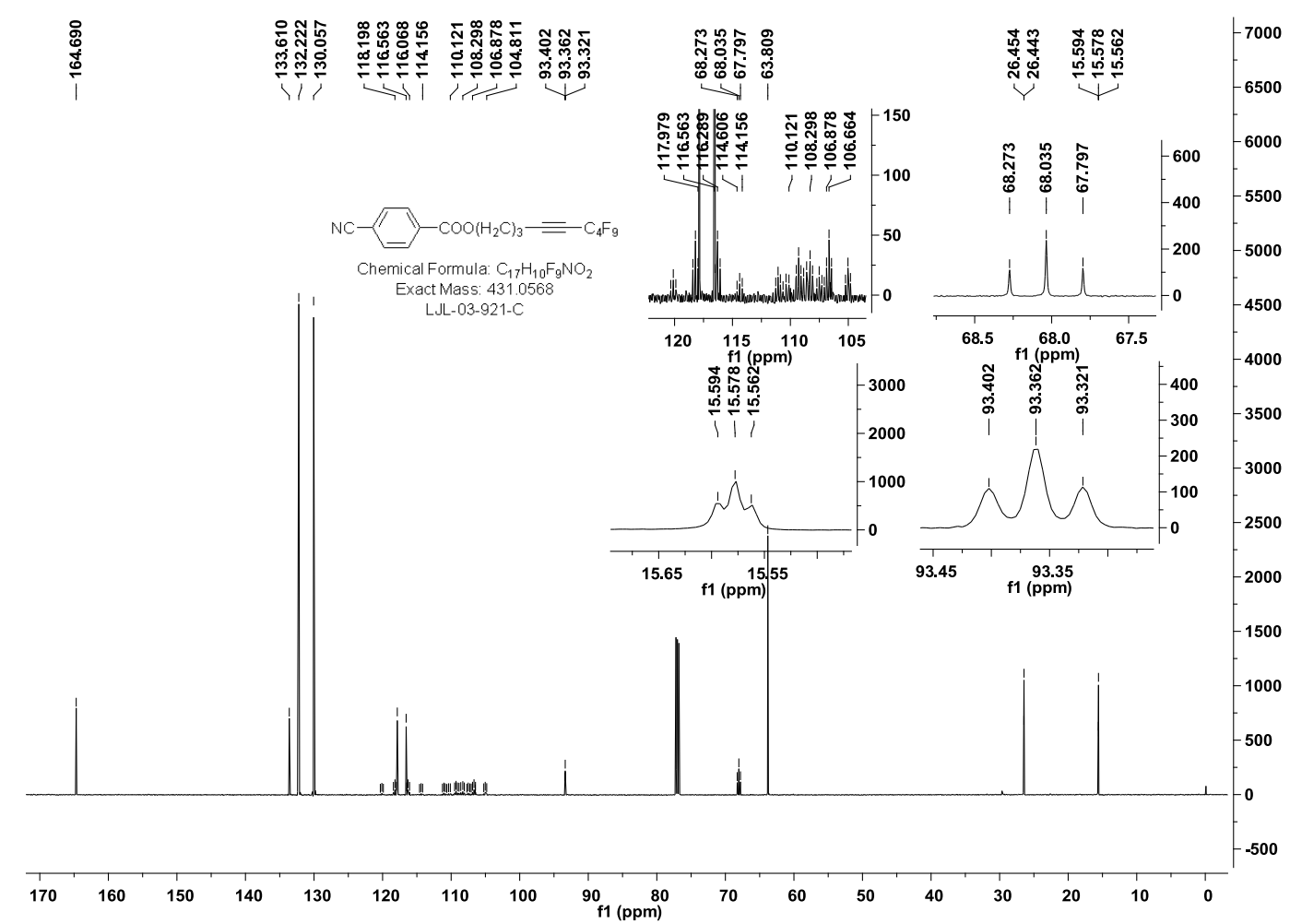

\section{6,6,7,7,8,8,9,9,9-Nonafluoronon-4-yn-1-yl 4-nitrobenzoate (6p)}

${ }^{1} \mathbf{H}$ NMR $\left(600 \mathrm{MHz}, \mathrm{CDCl}_{3}\right)$

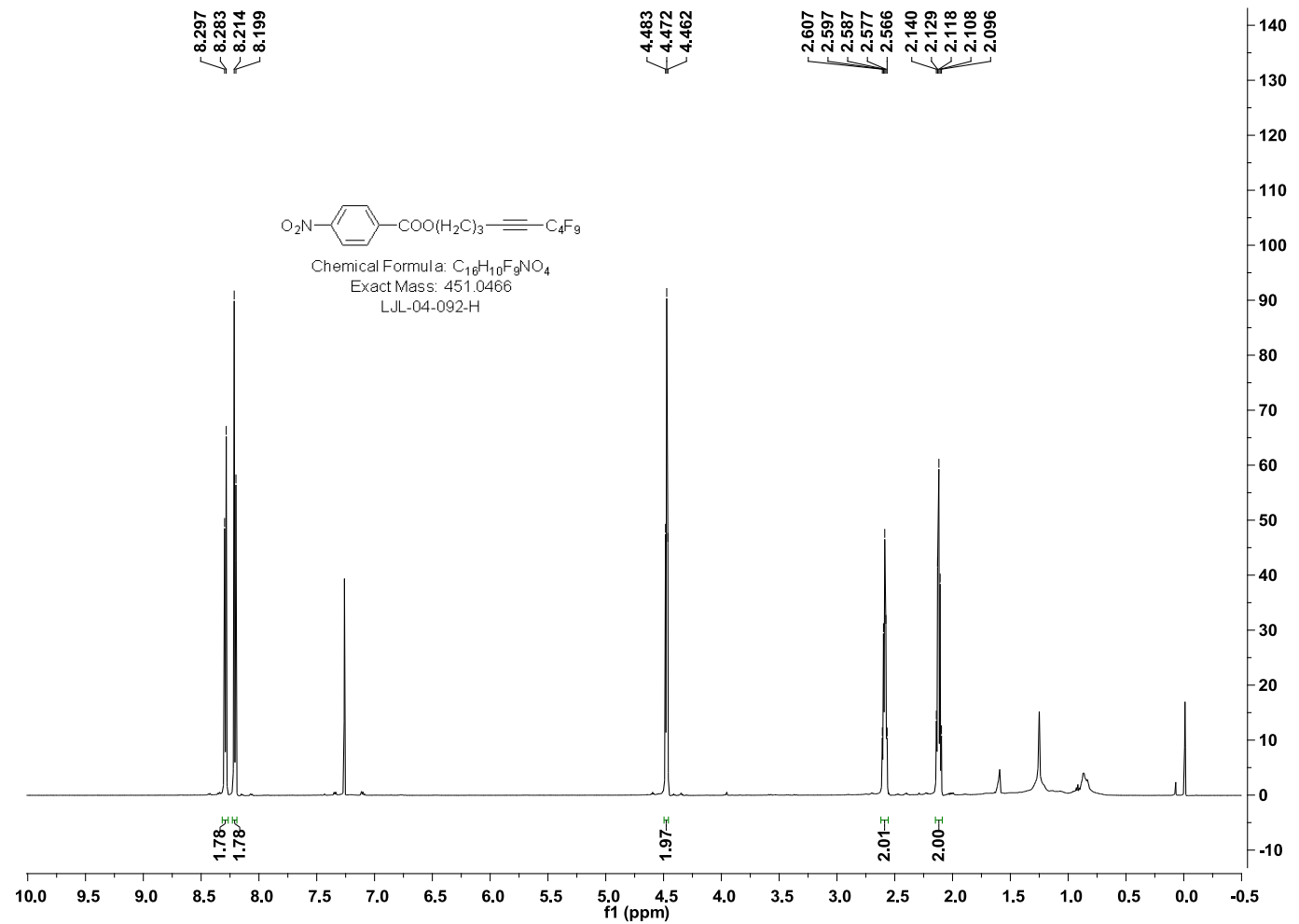


${ }^{19}$ F NMR $\left(564 \mathrm{MHz}, \mathrm{CDCl}_{3}\right)$

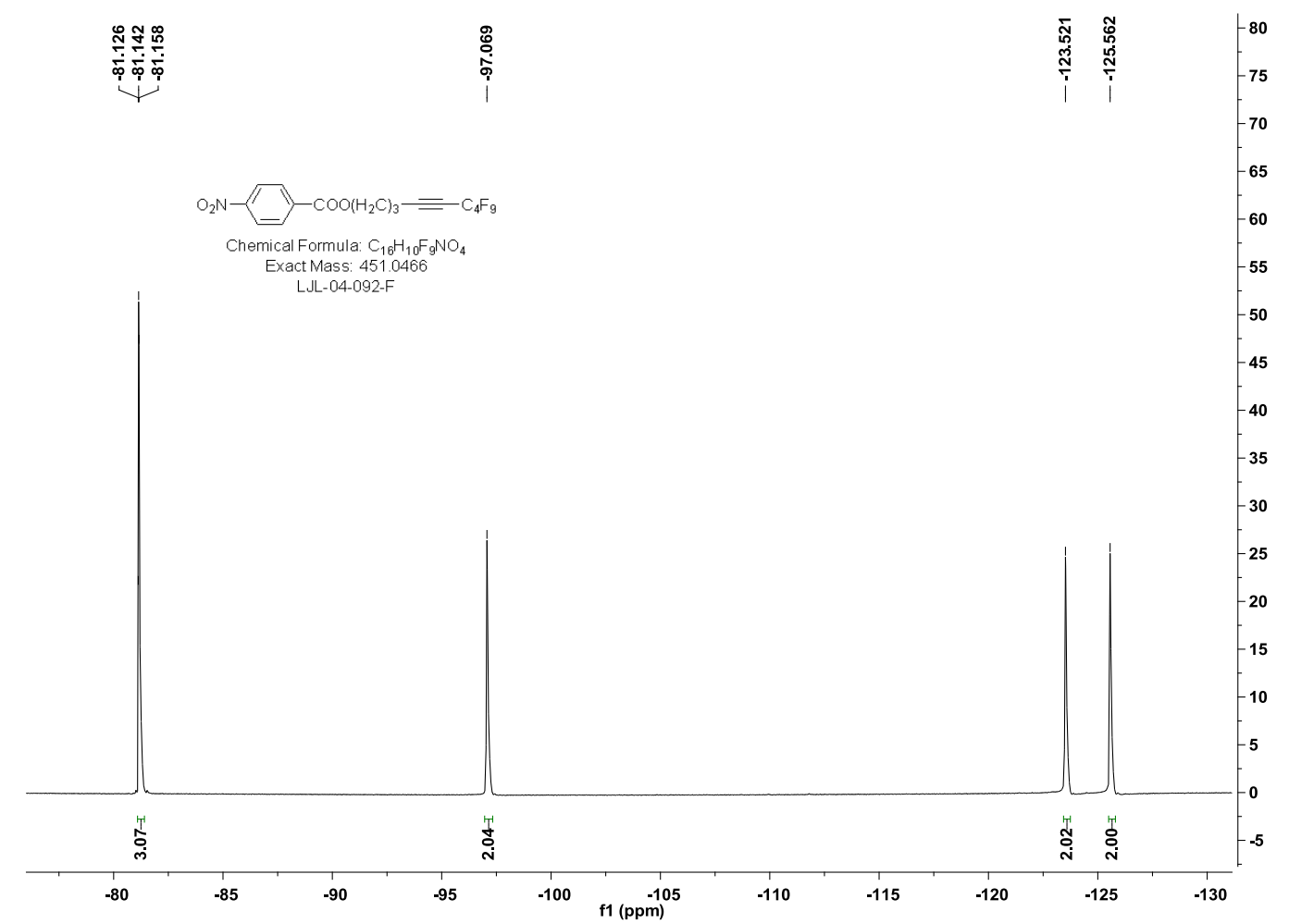

${ }^{13}$ C NMR (150.8 MHz, $\left.\mathrm{CDCl}_{3}\right)$

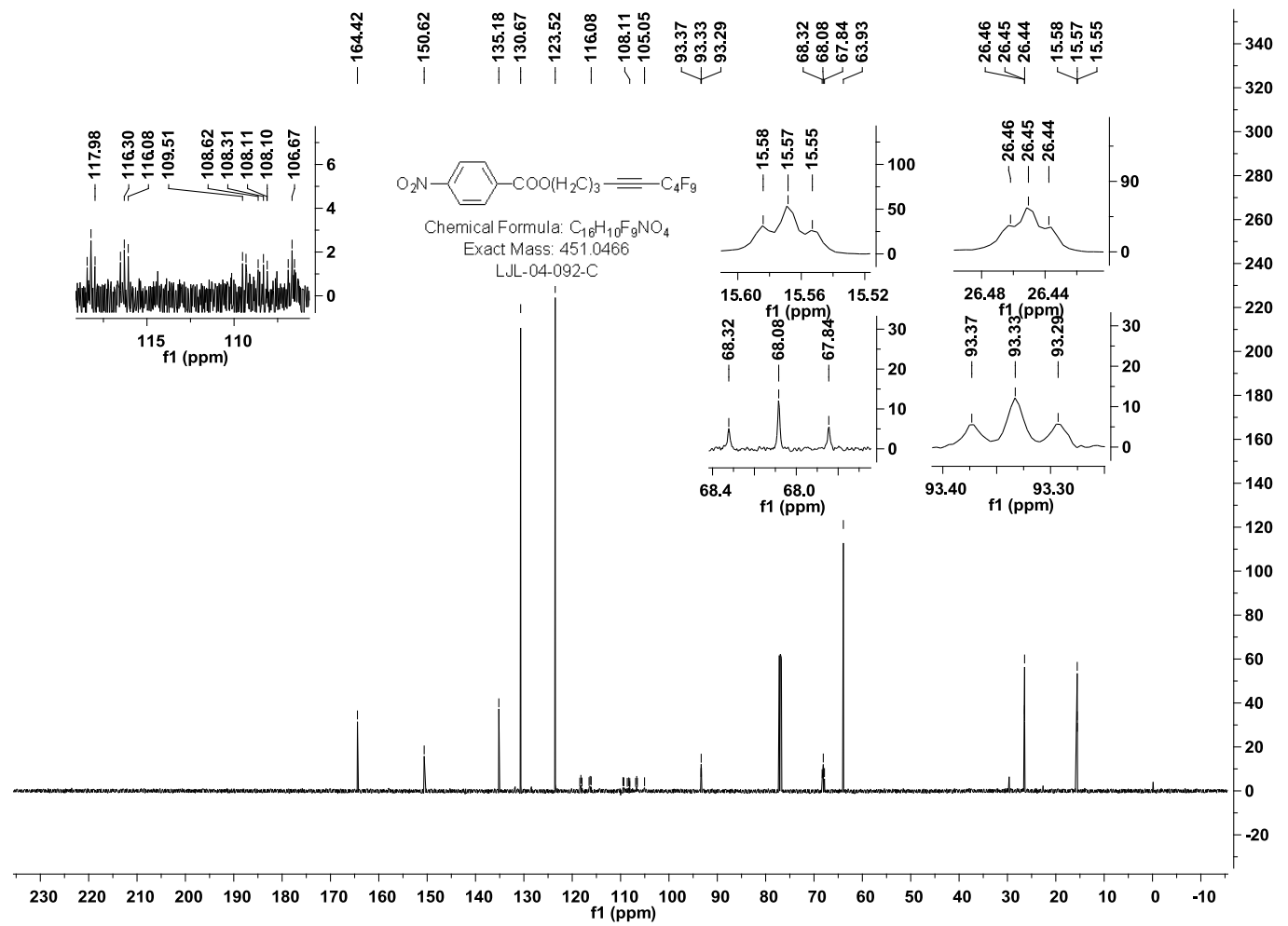


Diethyl (6,6,7,7,8,8,9,9,9-nonafluoronon-4-yn-1-yl) phosphate (6q)

${ }^{1} \mathbf{H}$ NMR $\left(600 \mathrm{MHz}, \mathrm{CDCl}_{3}\right)$

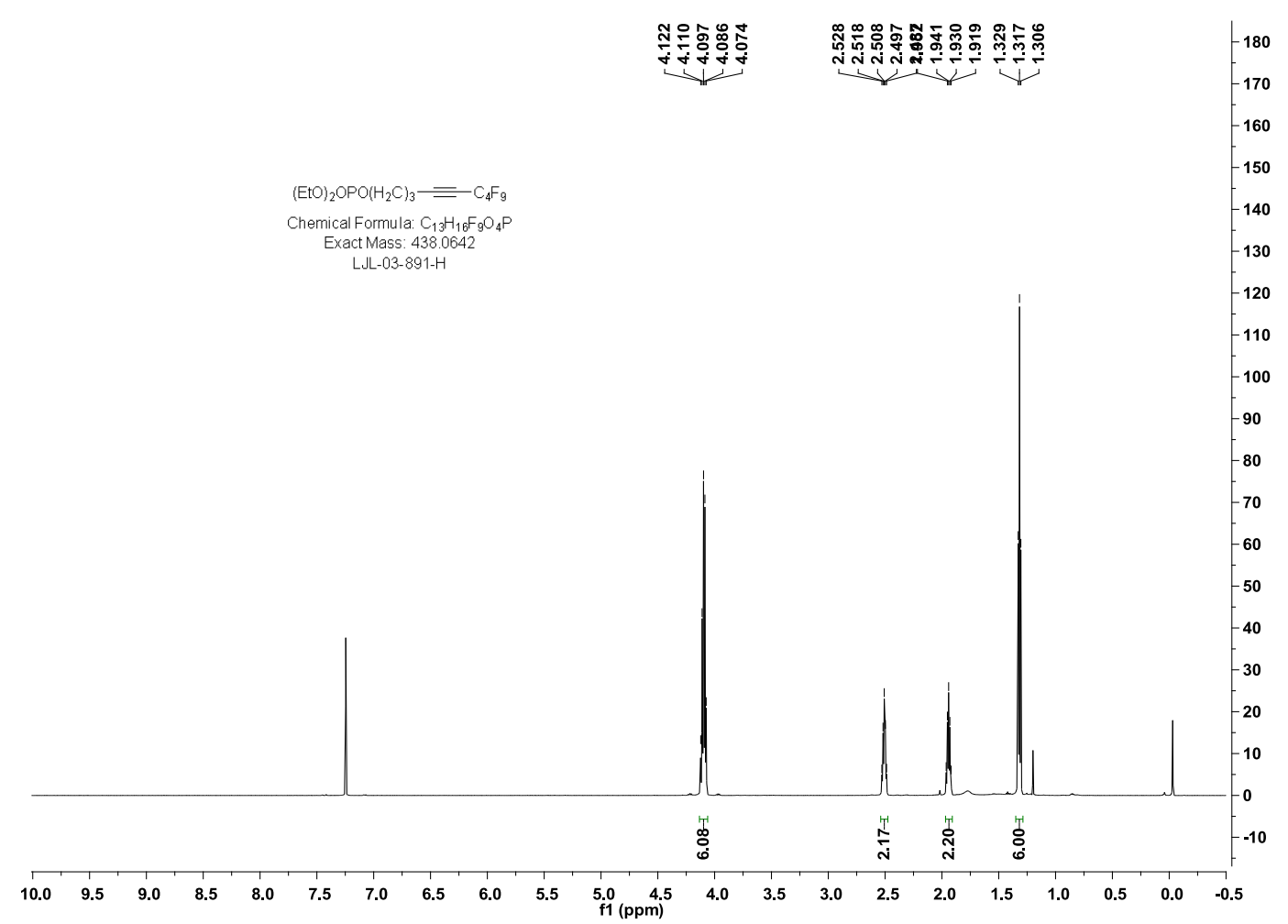

${ }^{19}$ F NMR $\left(564 \mathrm{MHz}, \mathrm{CDCl}_{3}\right)$

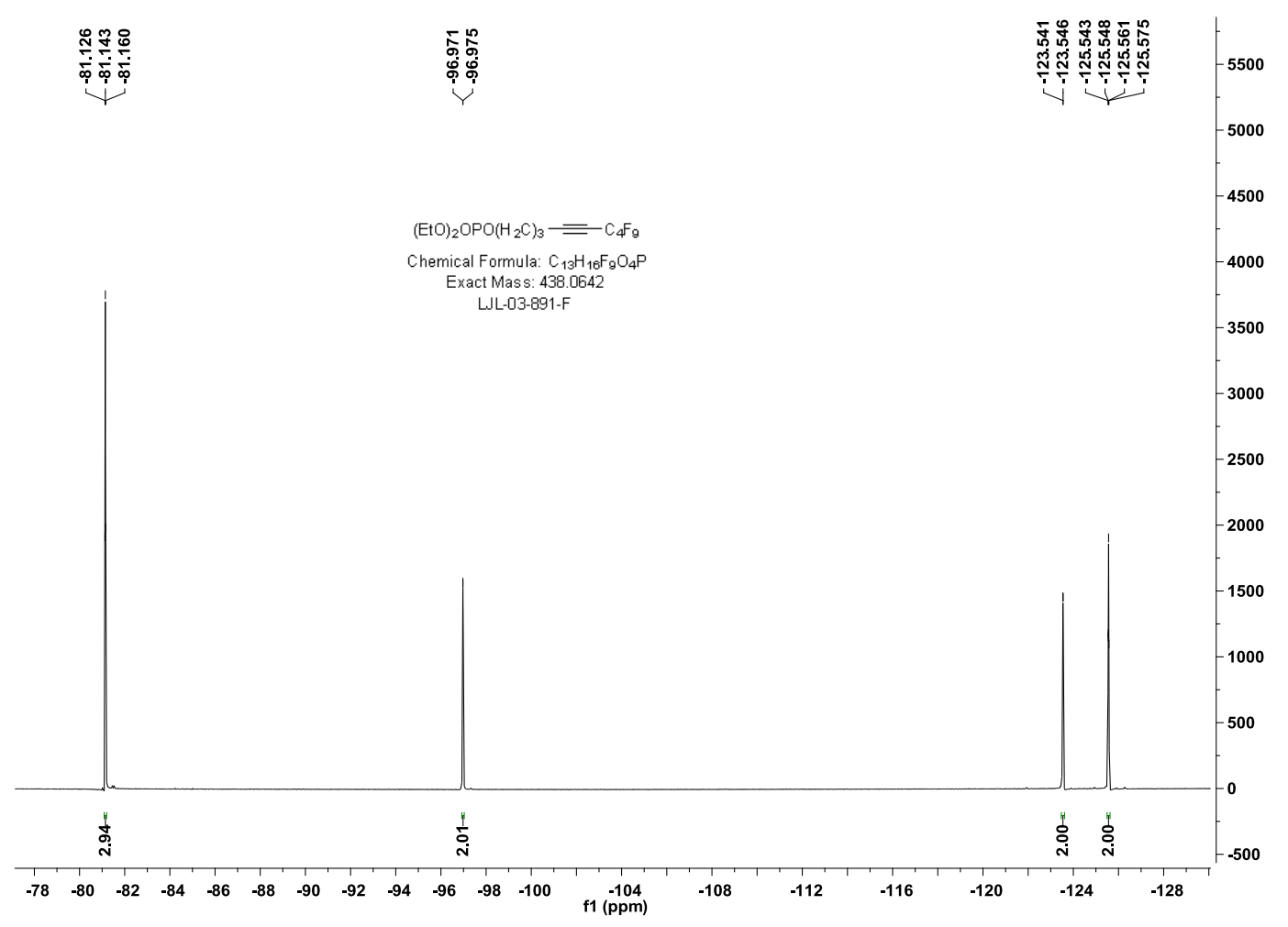


${ }^{13} \mathbf{C ~ N M R}\left(150.8 \mathrm{MHz}, \mathrm{CDCl}_{3}\right)$

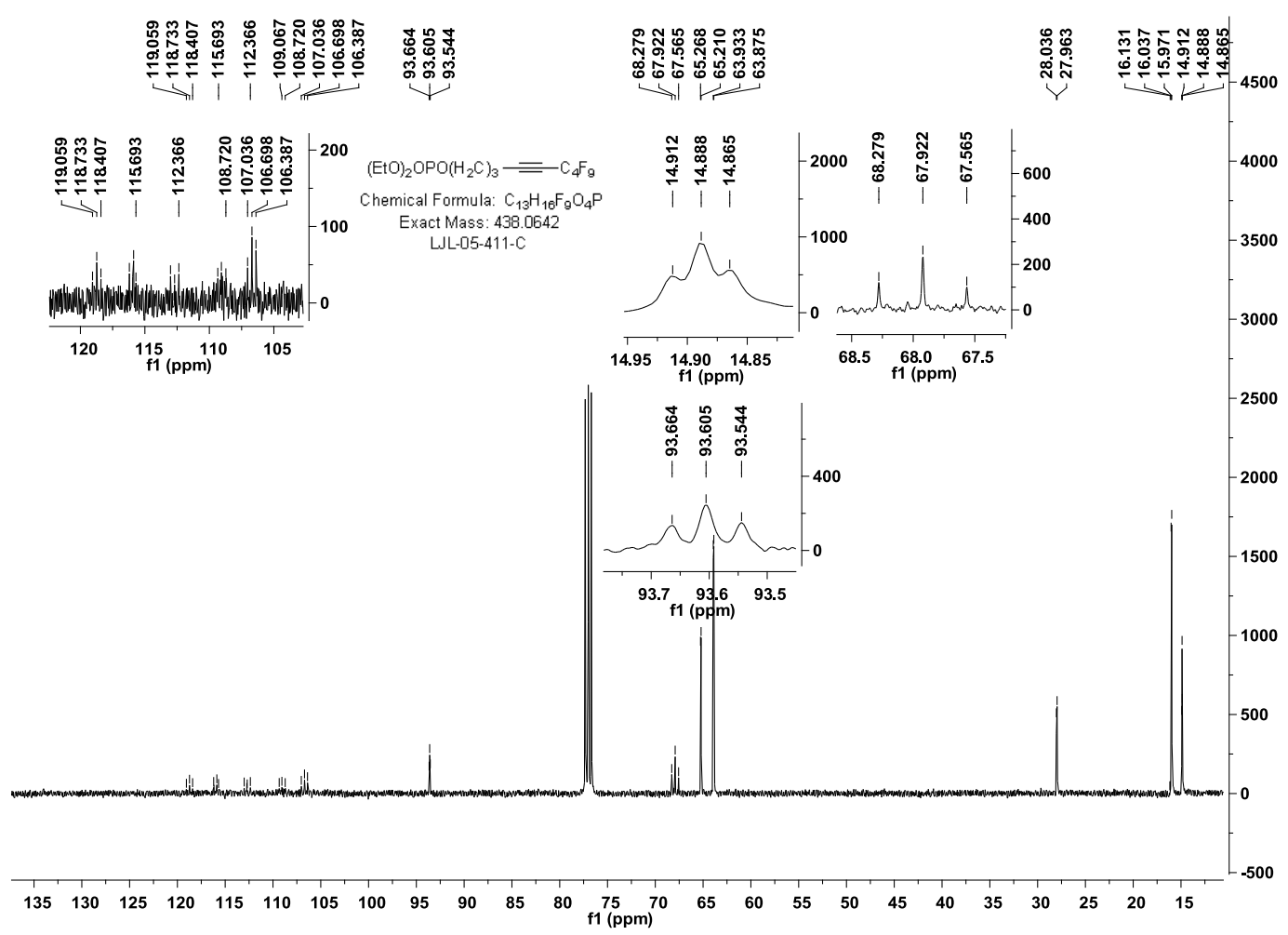

6,6,7,7,8,8,9,9,9-Nonafluoronon-4-yn-1-yl 4-methylbenzenesulfonate (6r)

${ }^{1} \mathbf{H}$ NMR $\left(600 \mathrm{MHz}, \mathrm{CDCl}_{3}\right)$

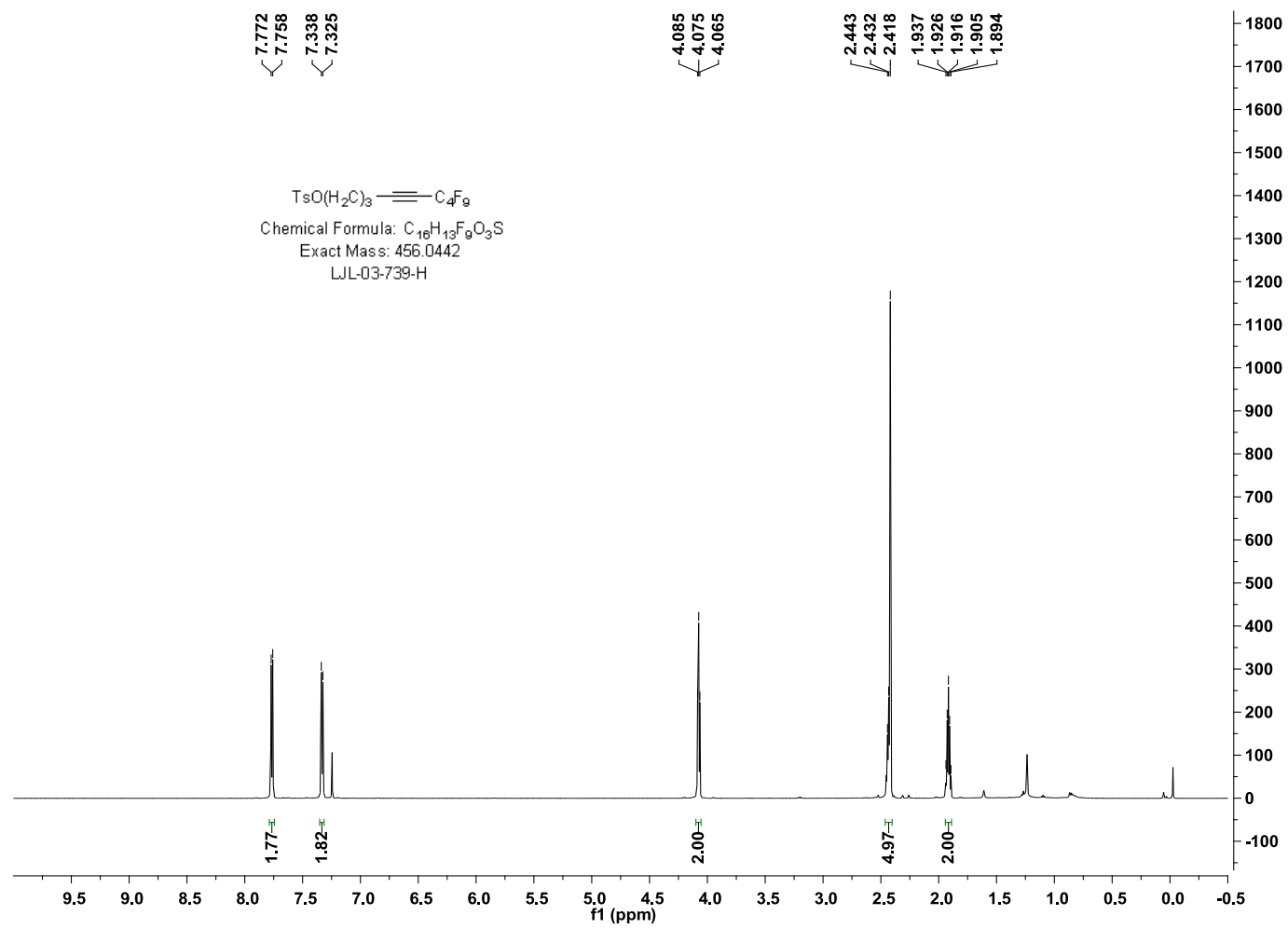


${ }^{19}$ F NMR $\left(564 \mathrm{MHz}, \mathrm{CDCl}_{3}\right)$

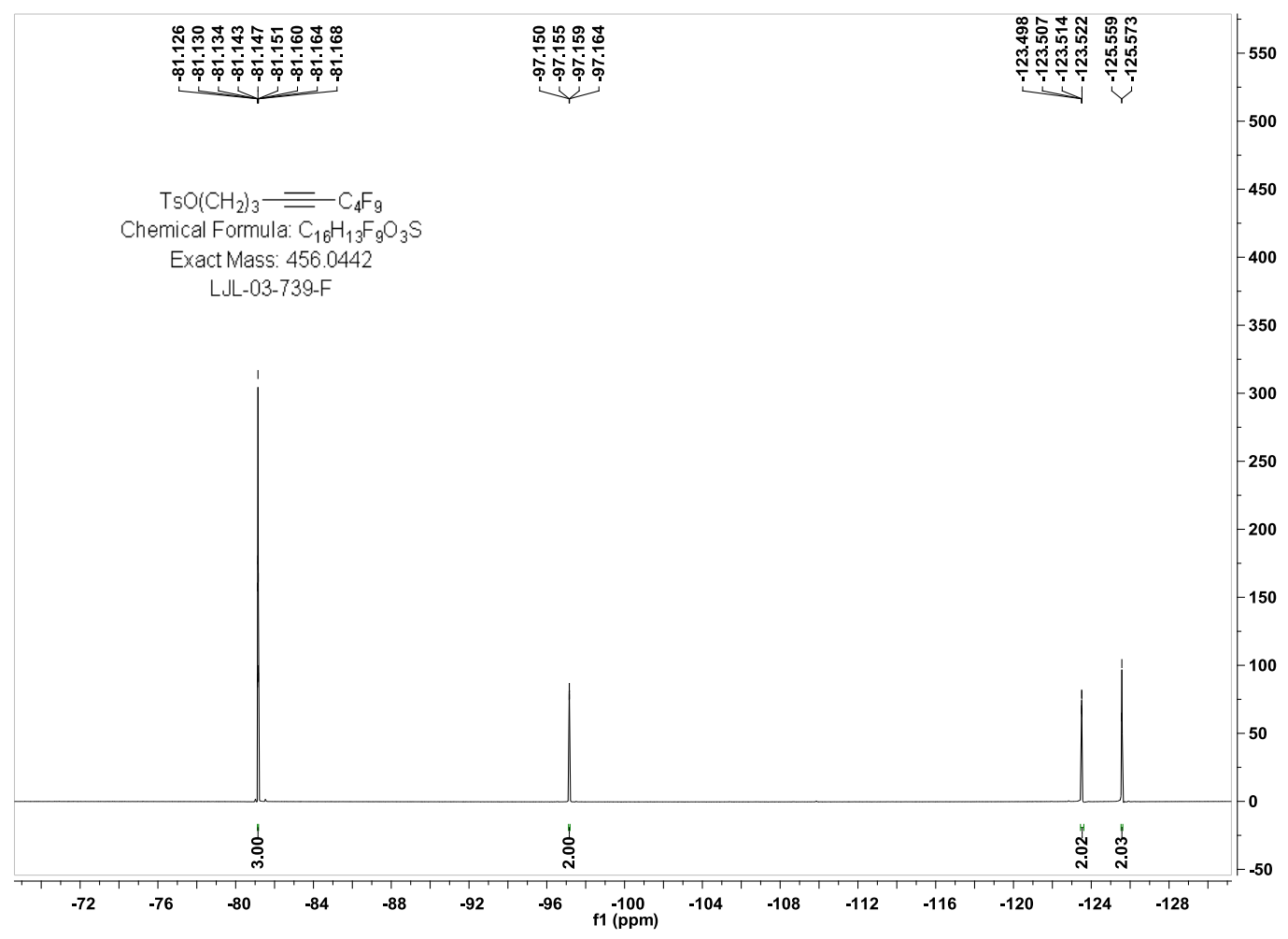

${ }^{13}$ C NMR (150.8 MHz, $\left.\mathrm{CDCl}_{3}\right)$

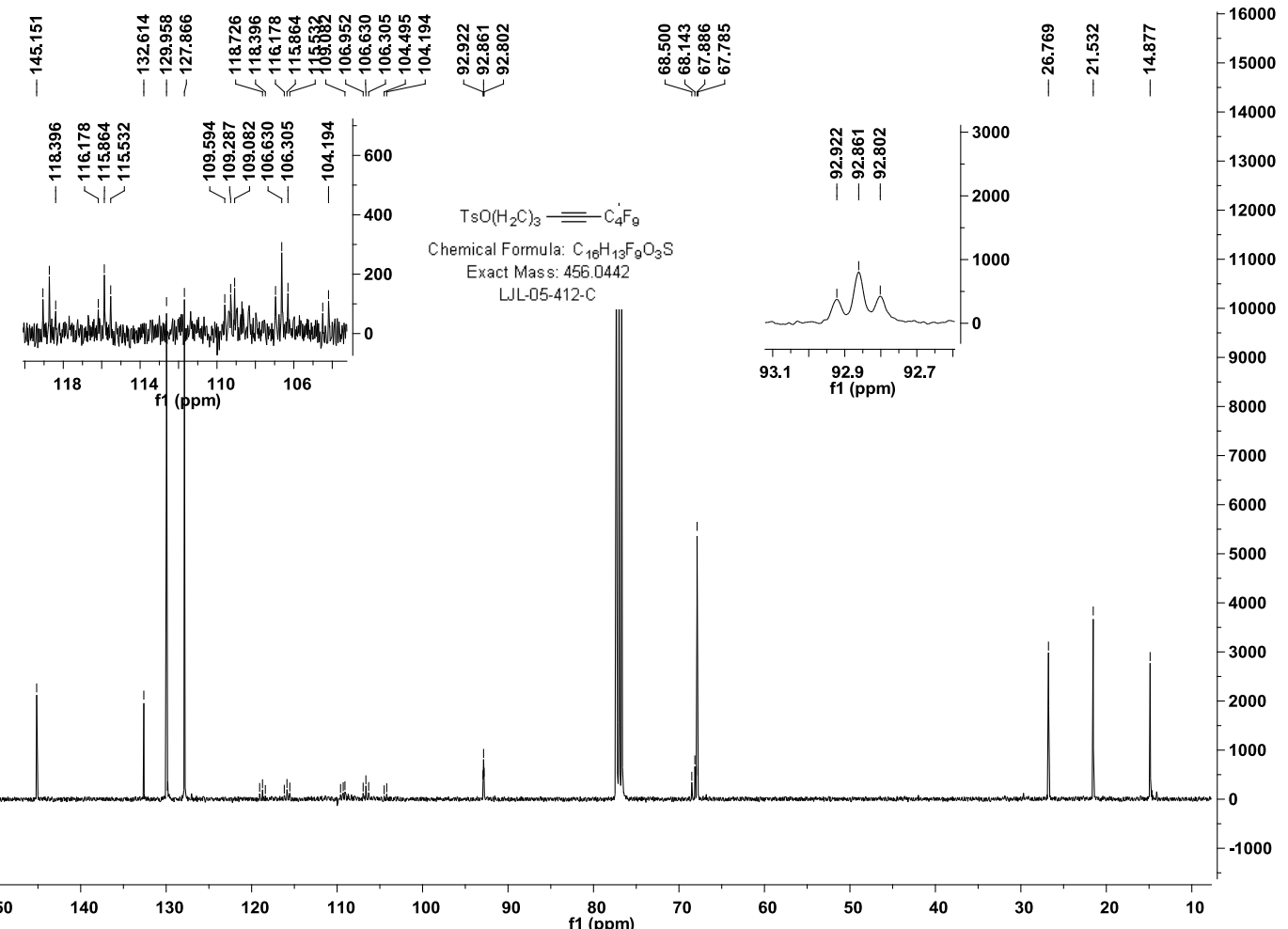


(8R,9S,13S,14S)-13-methyl-3-((4,4,5,5,6,6,7,7,7-nonafluorohept-2-yn-1-yl)oxy)-

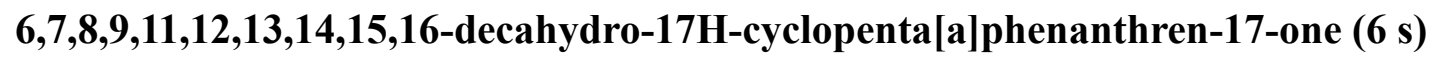
${ }^{1} \mathbf{H}$ NMR $\left(600 \mathrm{MHz}, \mathrm{CDCl}_{3}\right)$

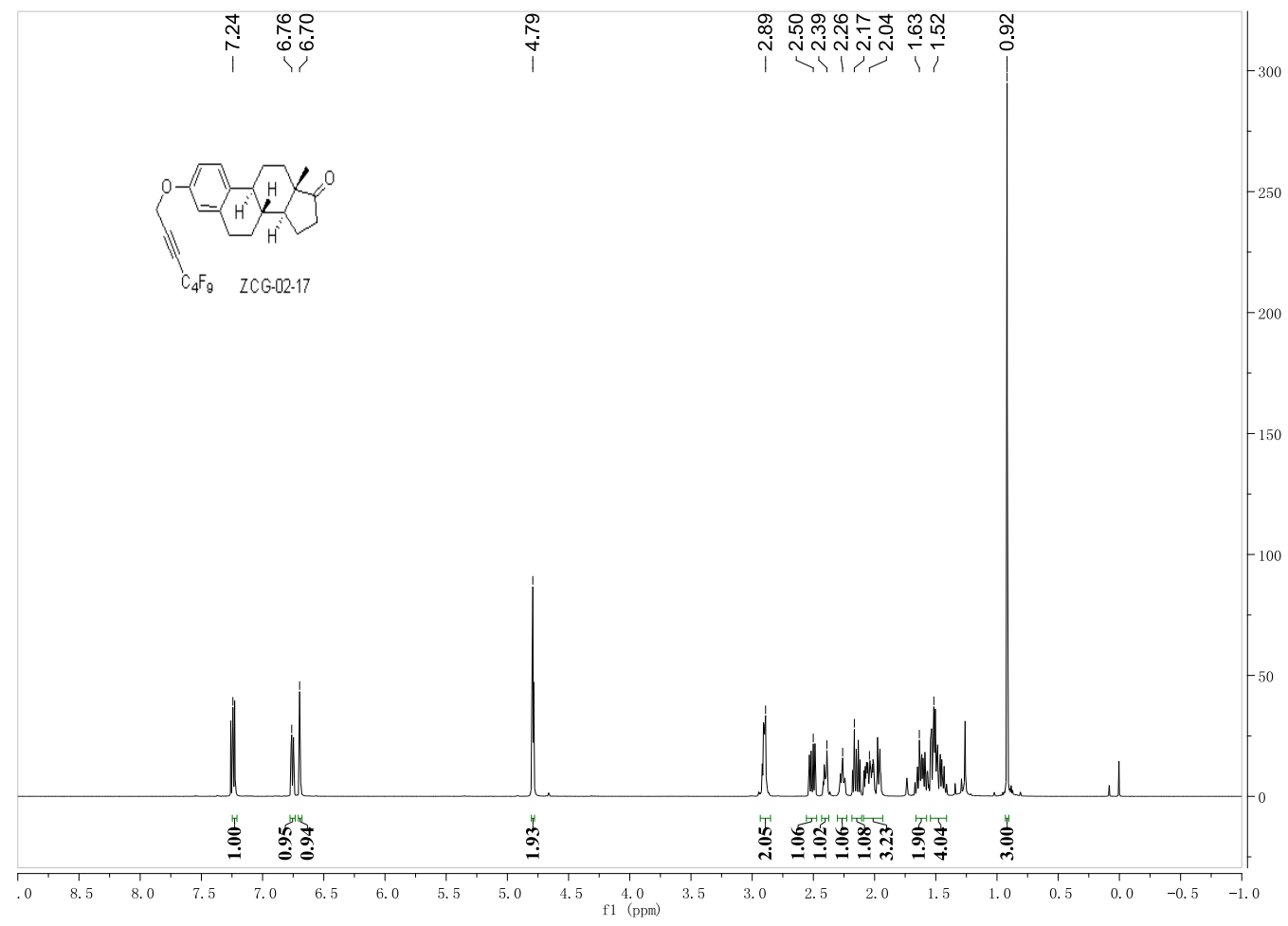

${ }^{19}$ F NMR (564 MHz, $\left.\mathrm{CDCl}_{3}\right)$

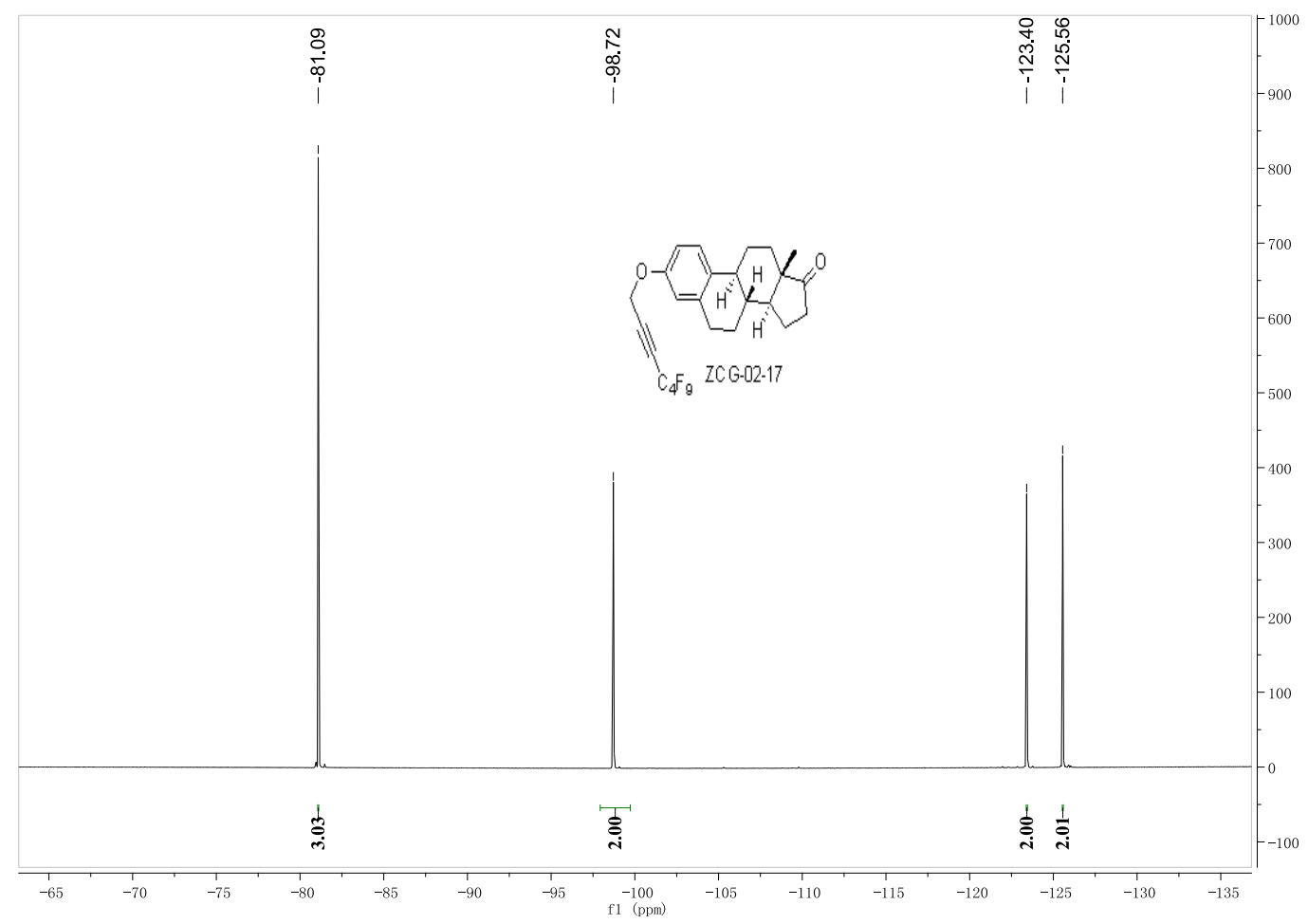


${ }^{13} \mathbf{C ~ N M R}\left(150.8 \mathrm{MHz}, \mathrm{CDCl}_{3}\right)$

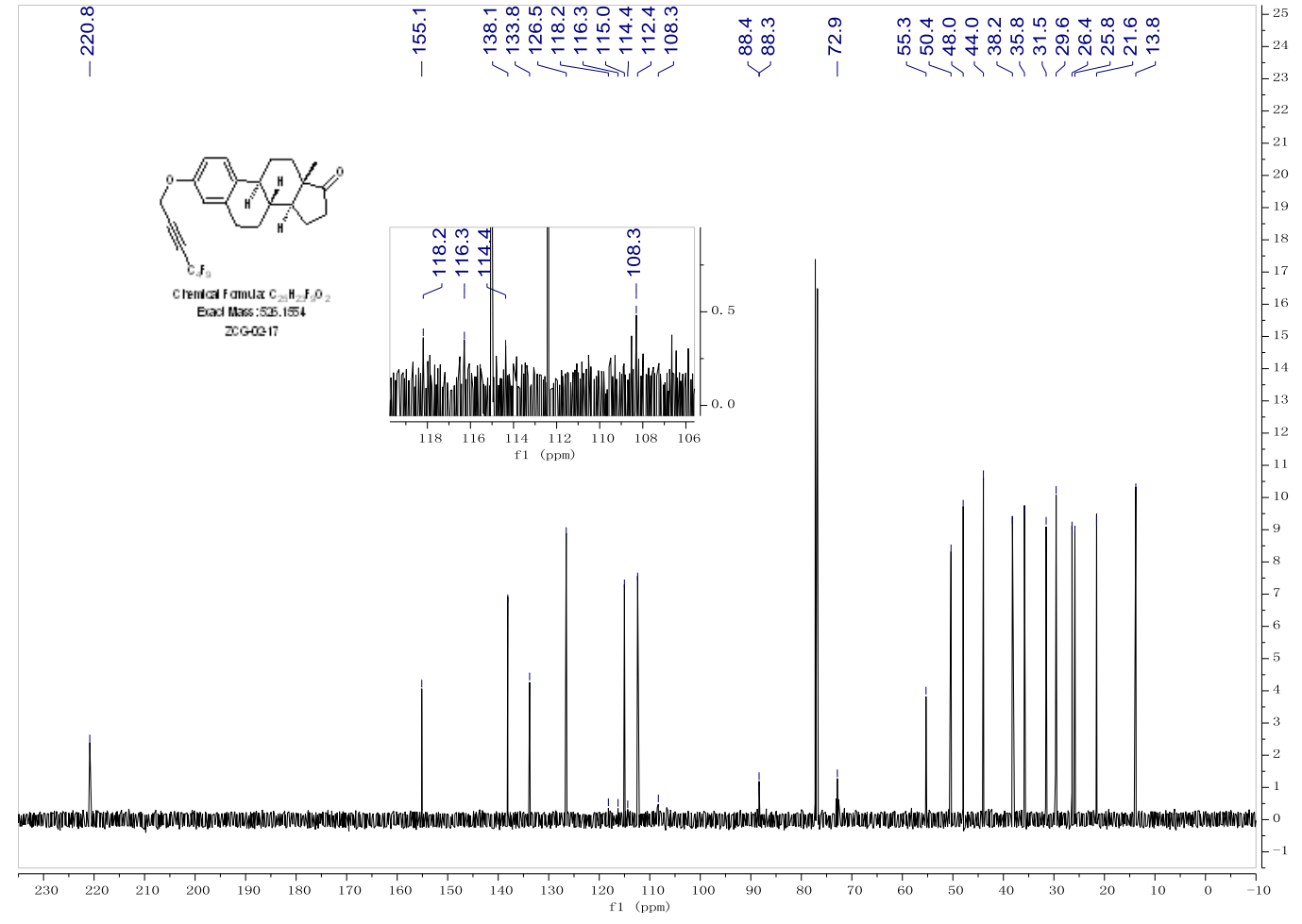

(1S,2R,4R)-1-isopropyl-4-methyl-2-((4,4,5,5,6,6,7,7,7-nonafluorohept-2-yn-1yl)oxy)cyclohexane (6t)

${ }^{1} \mathbf{H}$ NMR $\left(600 \mathrm{MHz}, \mathrm{CDCl}_{3}\right)$

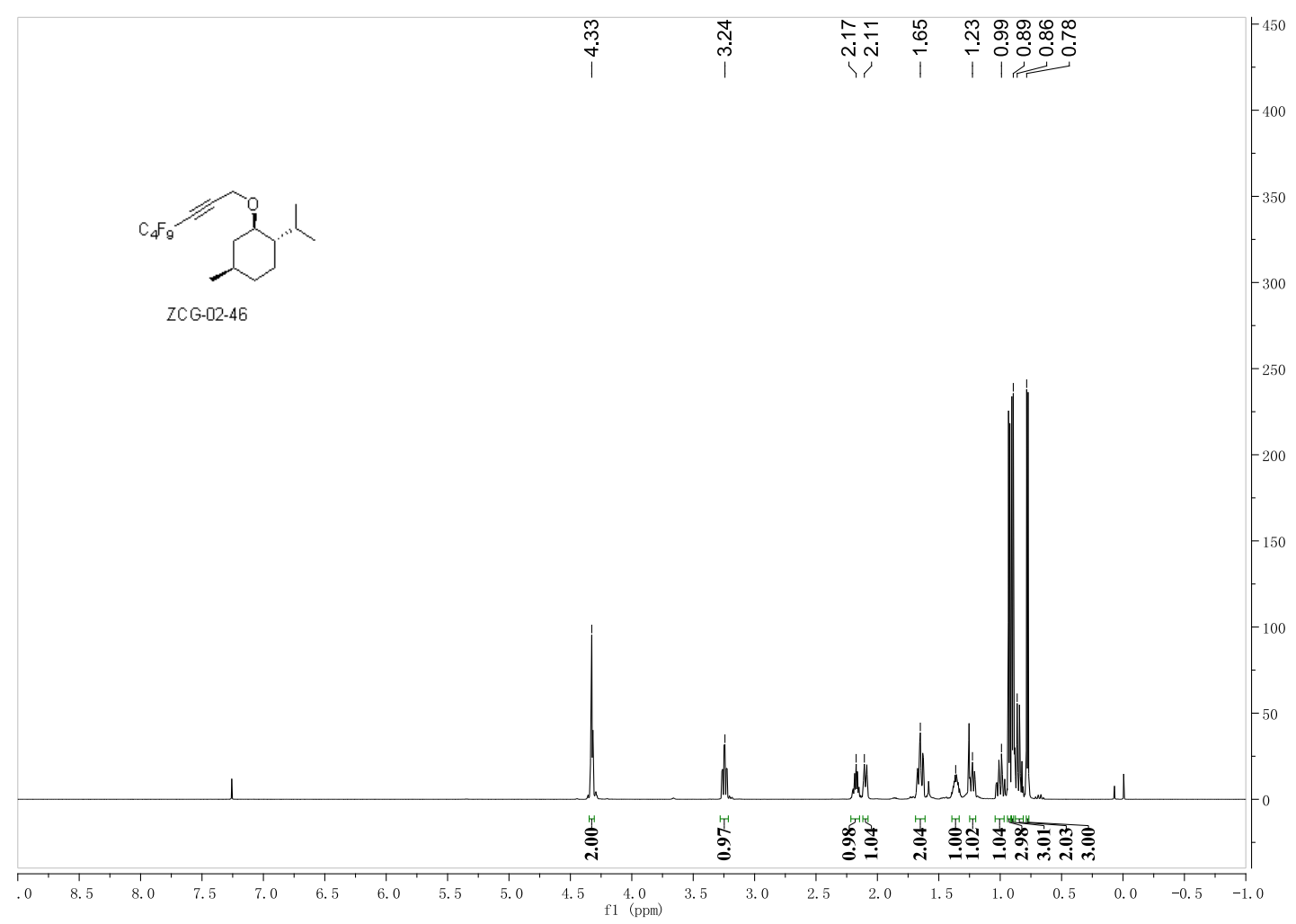


${ }^{19}$ F NMR $\left(564 \mathrm{MHz}, \mathrm{CDCl}_{3}\right)$

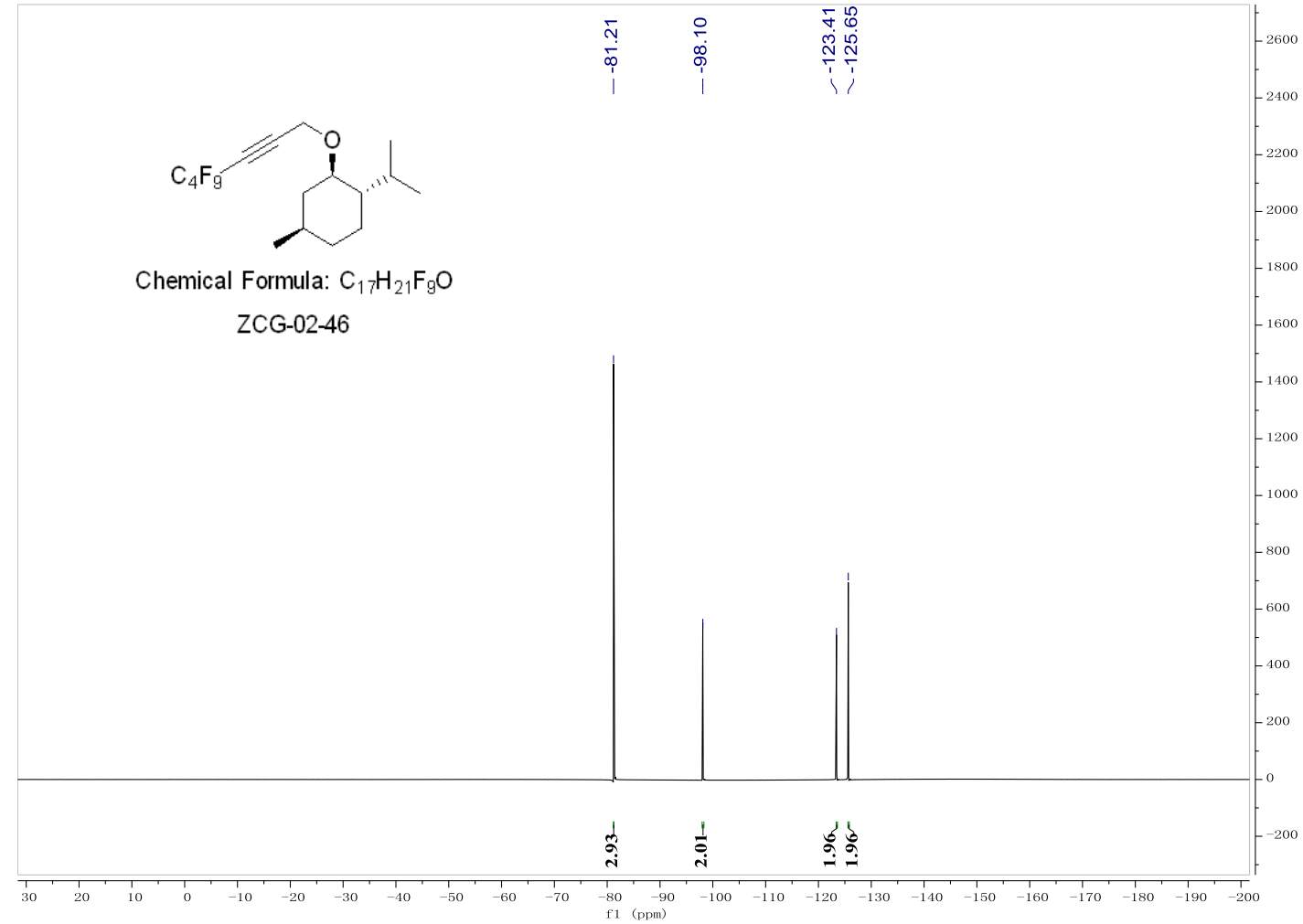

${ }^{13}$ C NMR (150.8 MHz, $\left.\mathrm{CDCl}_{3}\right)$

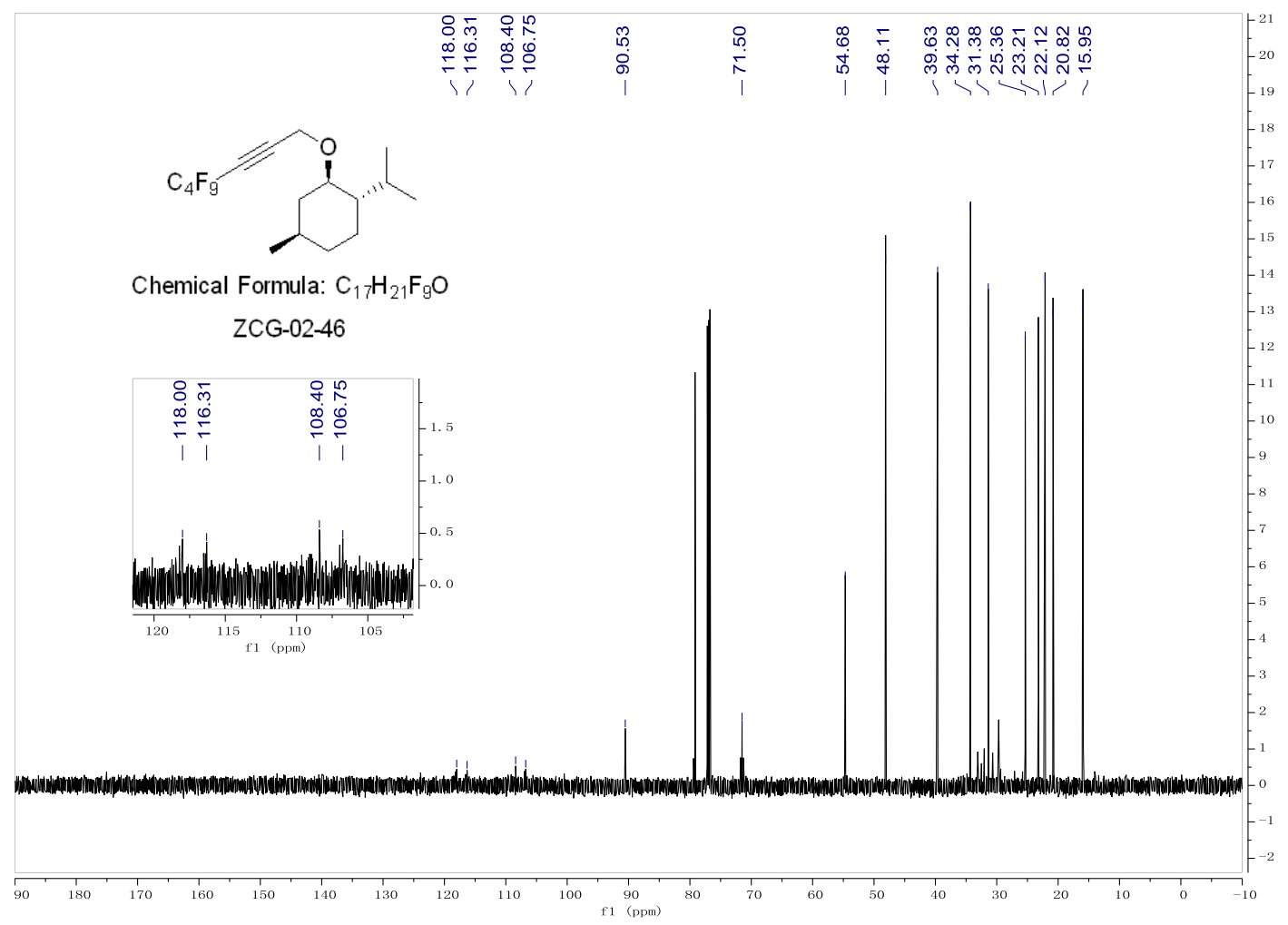


1-Pentyl-4-(perfluoropent-1-yn-1-yl)benzene (5b)

${ }^{1} \mathbf{H}$ NMR (600 MHz, $\left.\mathrm{CDCl}_{3}\right)$

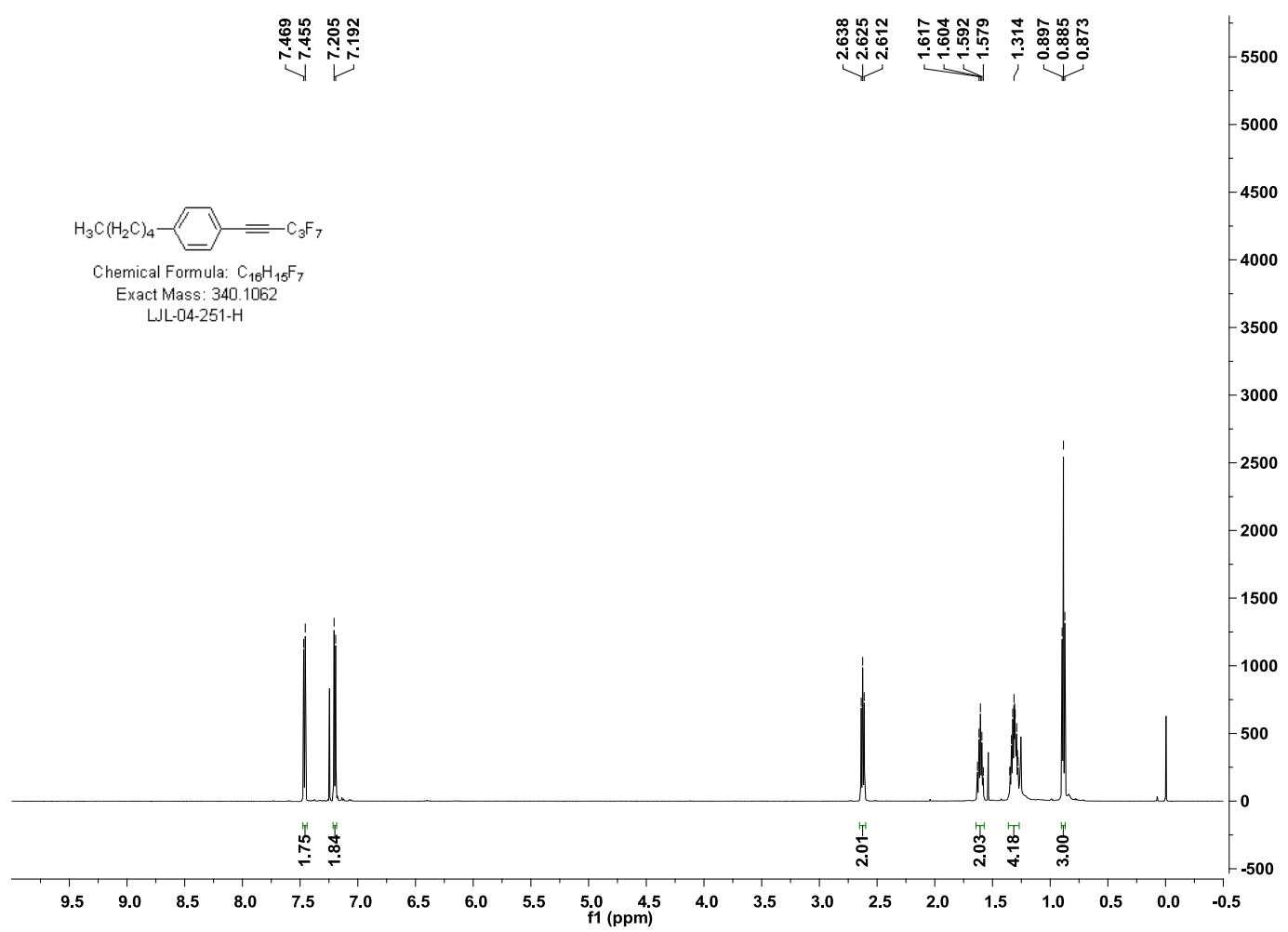

${ }^{19}$ F NMR (564 MHz, $\left.\mathrm{CDCl}_{3}\right)$

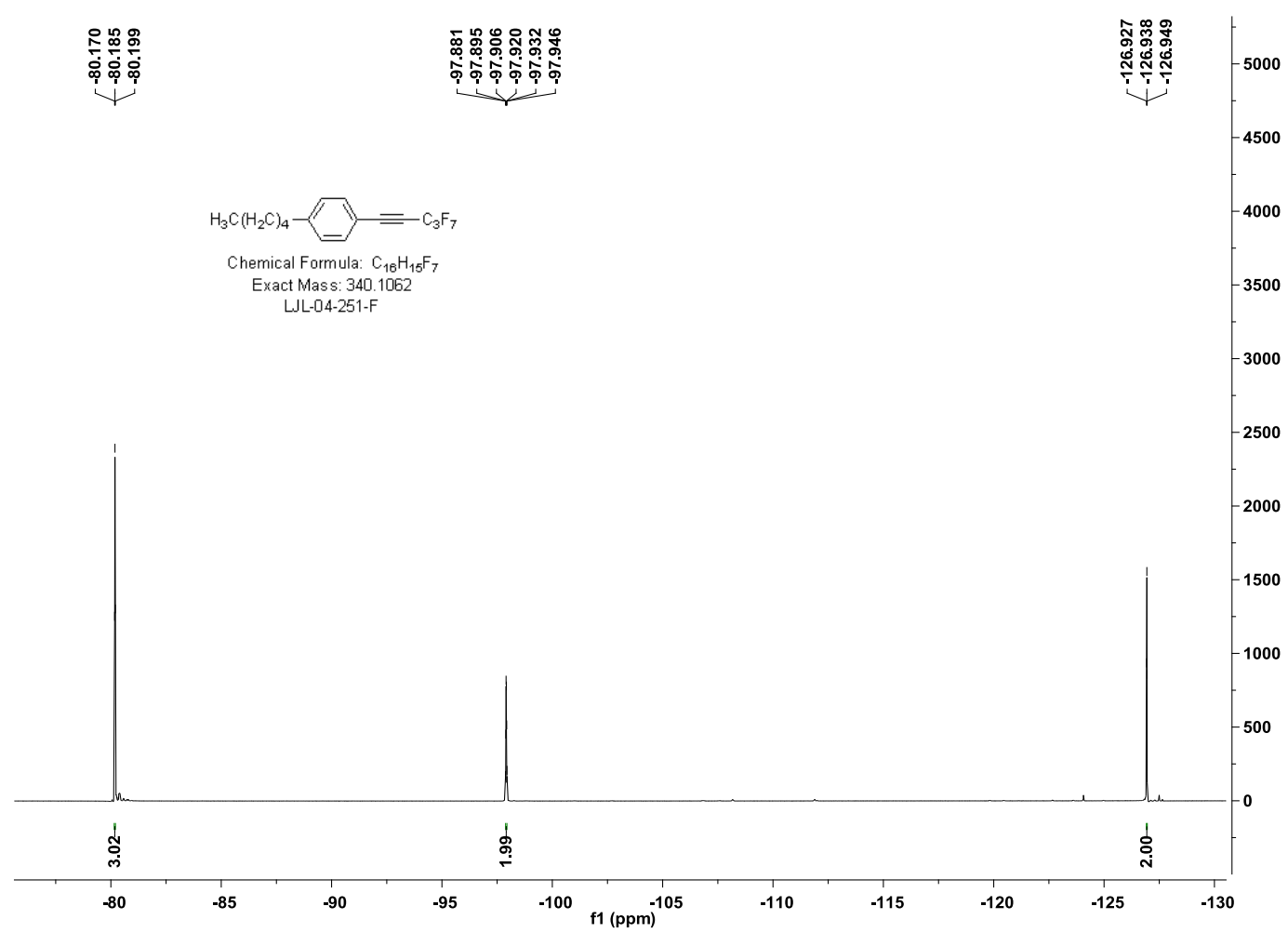


${ }^{13} \mathbf{C ~ N M R}\left(150.8 \mathrm{MHz}, \mathrm{CDCl}_{3}\right)$

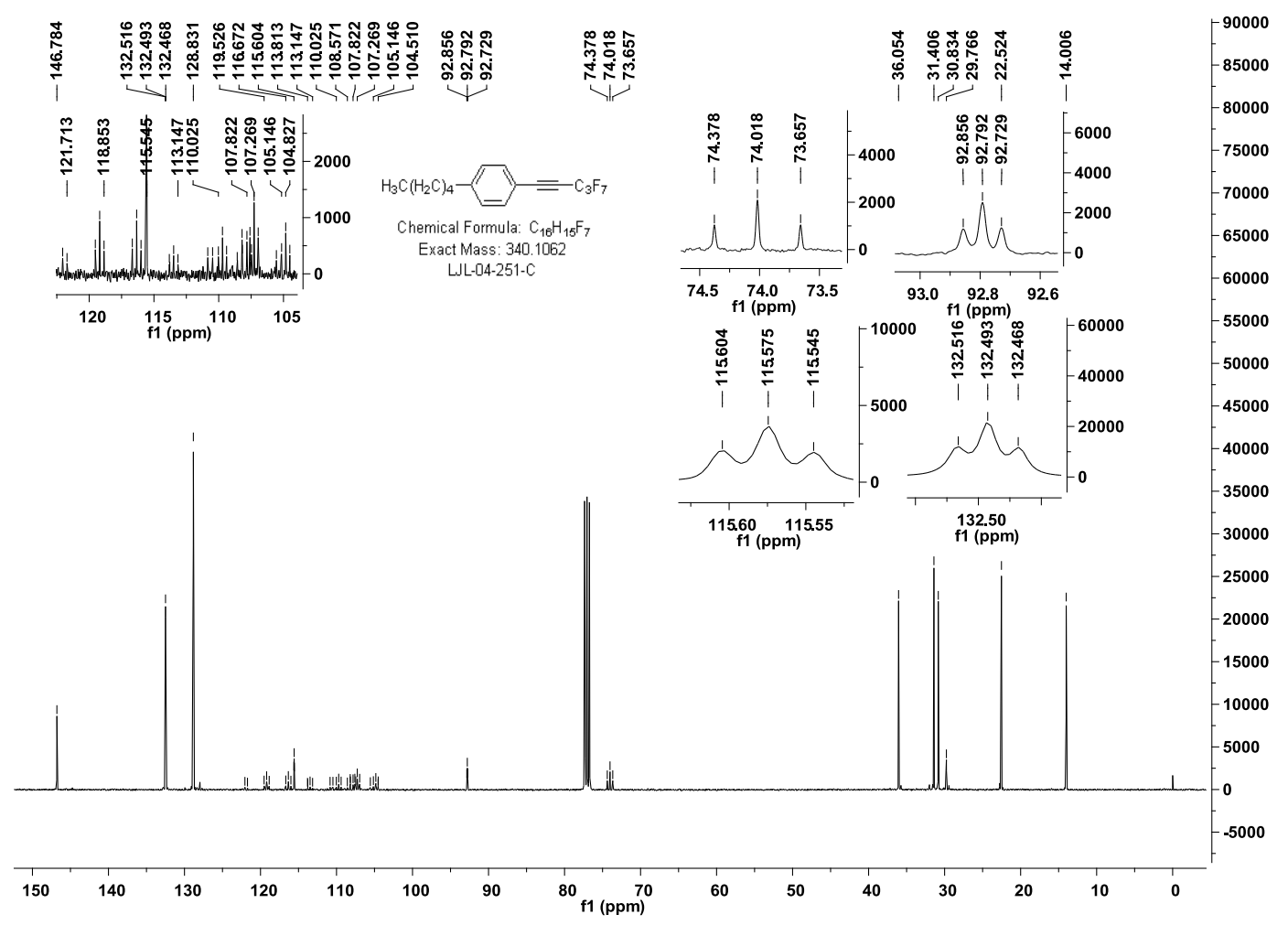

1-Chloro-4-(perfluoropent-1-yn-1-yl)benzene (5h)

${ }^{1} \mathbf{H}$ NMR (600 MHz, $\left.\mathrm{CDCl}_{3}\right)$

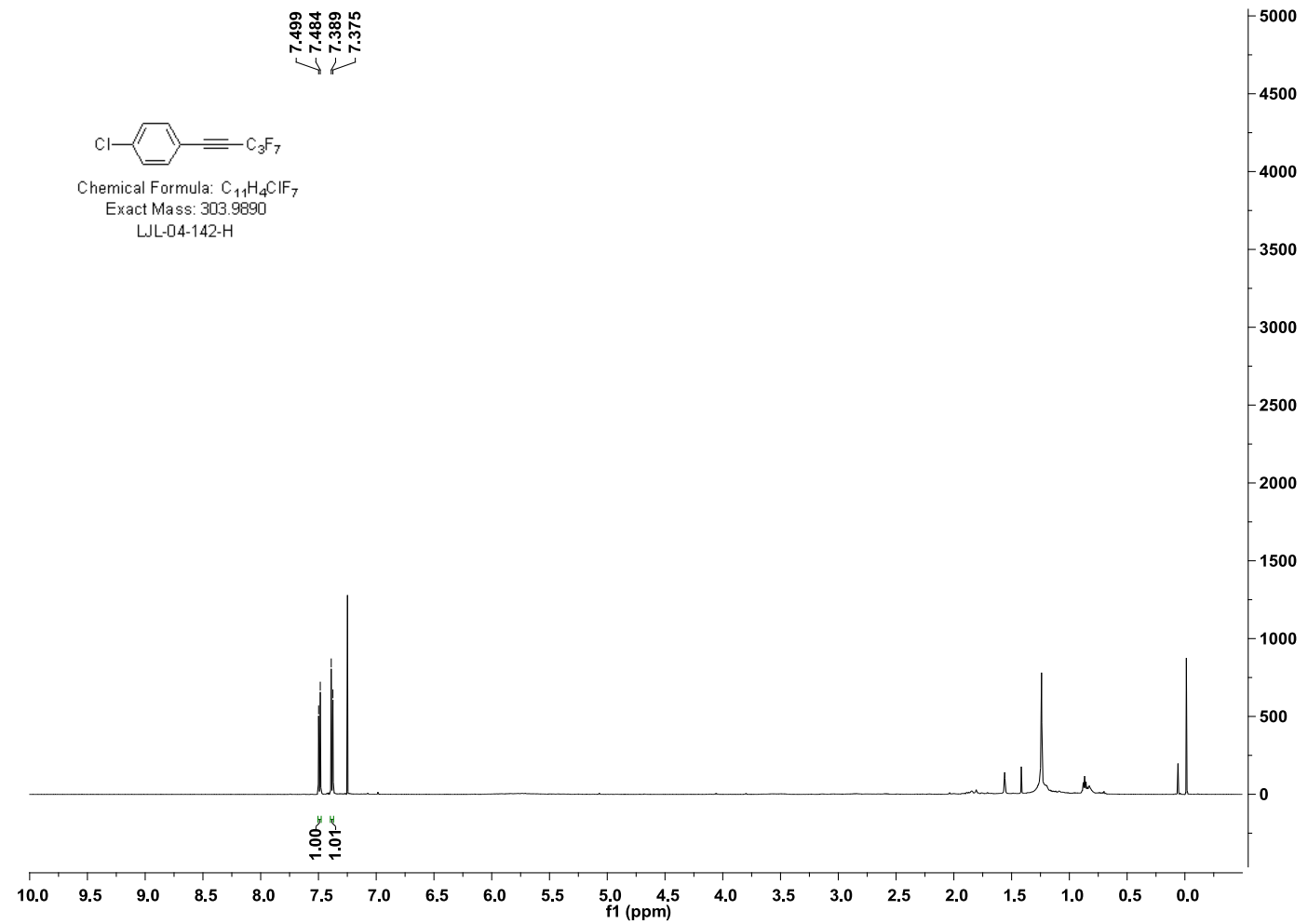


${ }^{19}$ F NMR $\left(564 \mathrm{MHz}, \mathrm{CDCl}_{3}\right)$

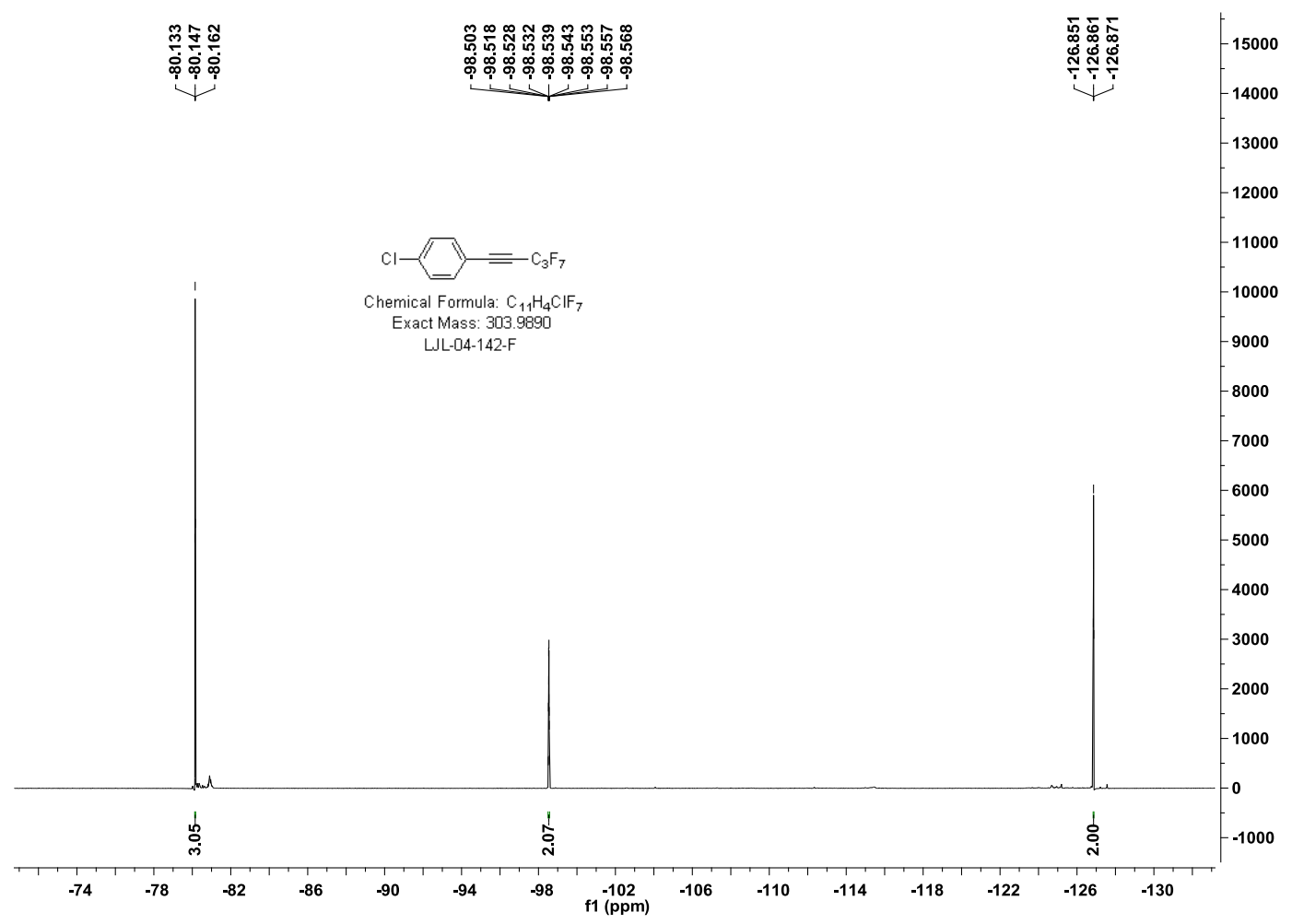

${ }^{13}$ C NMR (150.8 MHz, $\left.\mathrm{CDCl}_{3}\right)$

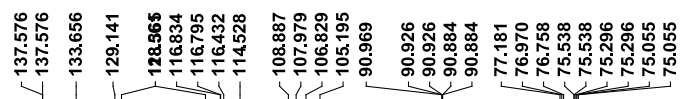
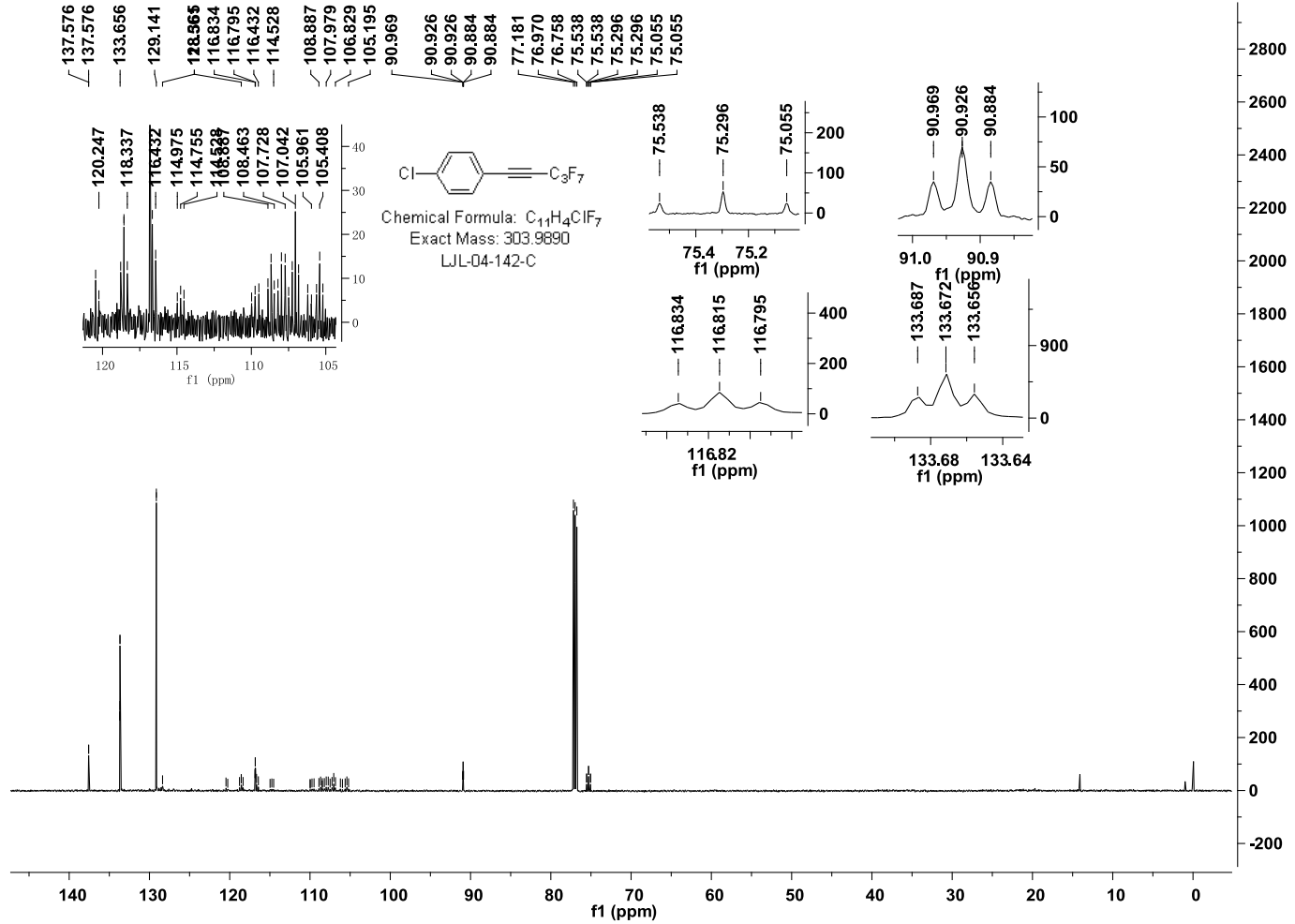
1,1,1,2,2,3,3-Heptafluoropentadec-4-yne (5l)

${ }^{1} \mathbf{H}$ NMR (600 MHz, $\left.\mathrm{CDCl}_{3}\right)$

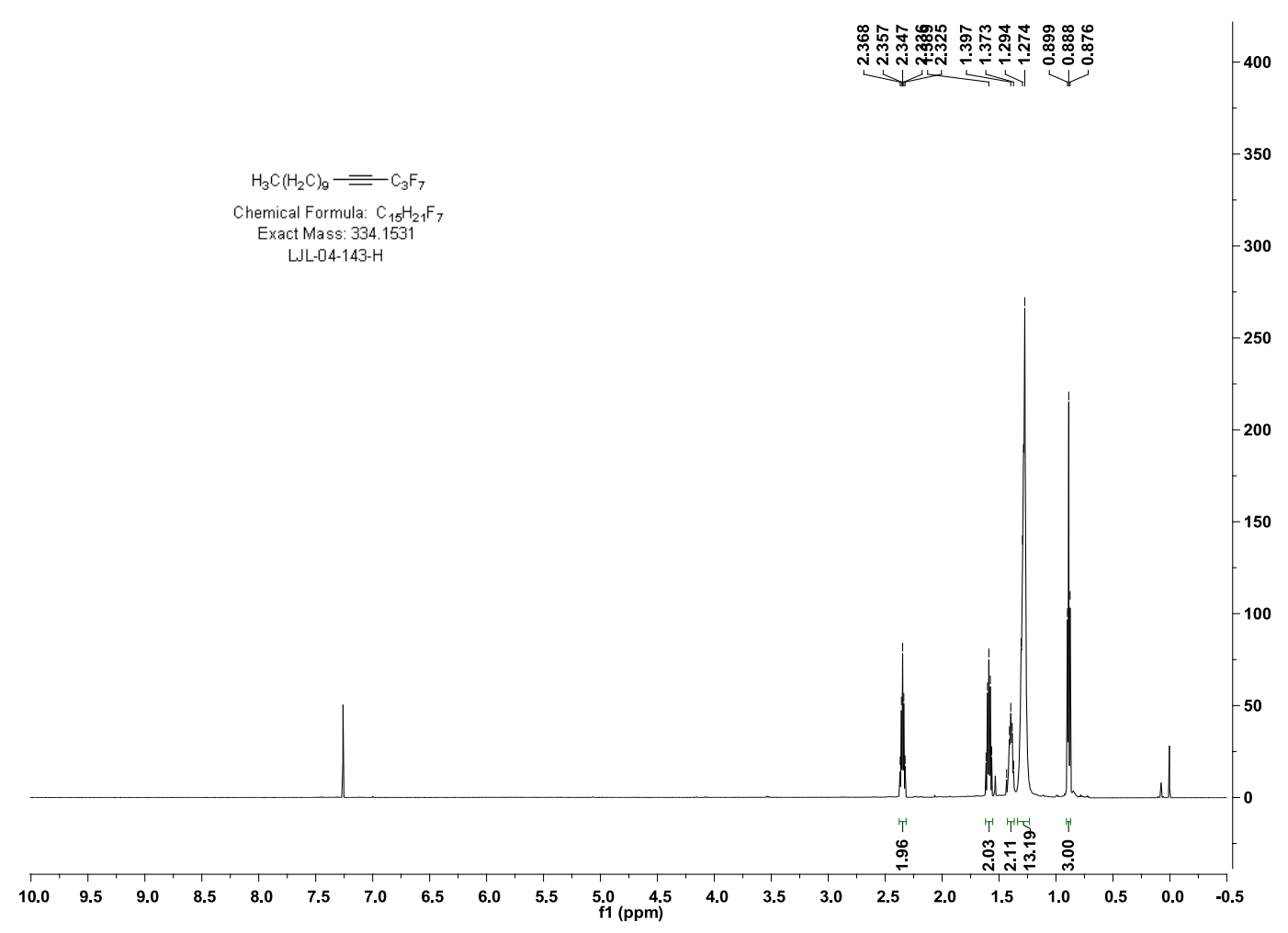

${ }^{19}$ F NMR (564 MHz, $\left.\mathrm{CDCl}_{3}\right)$

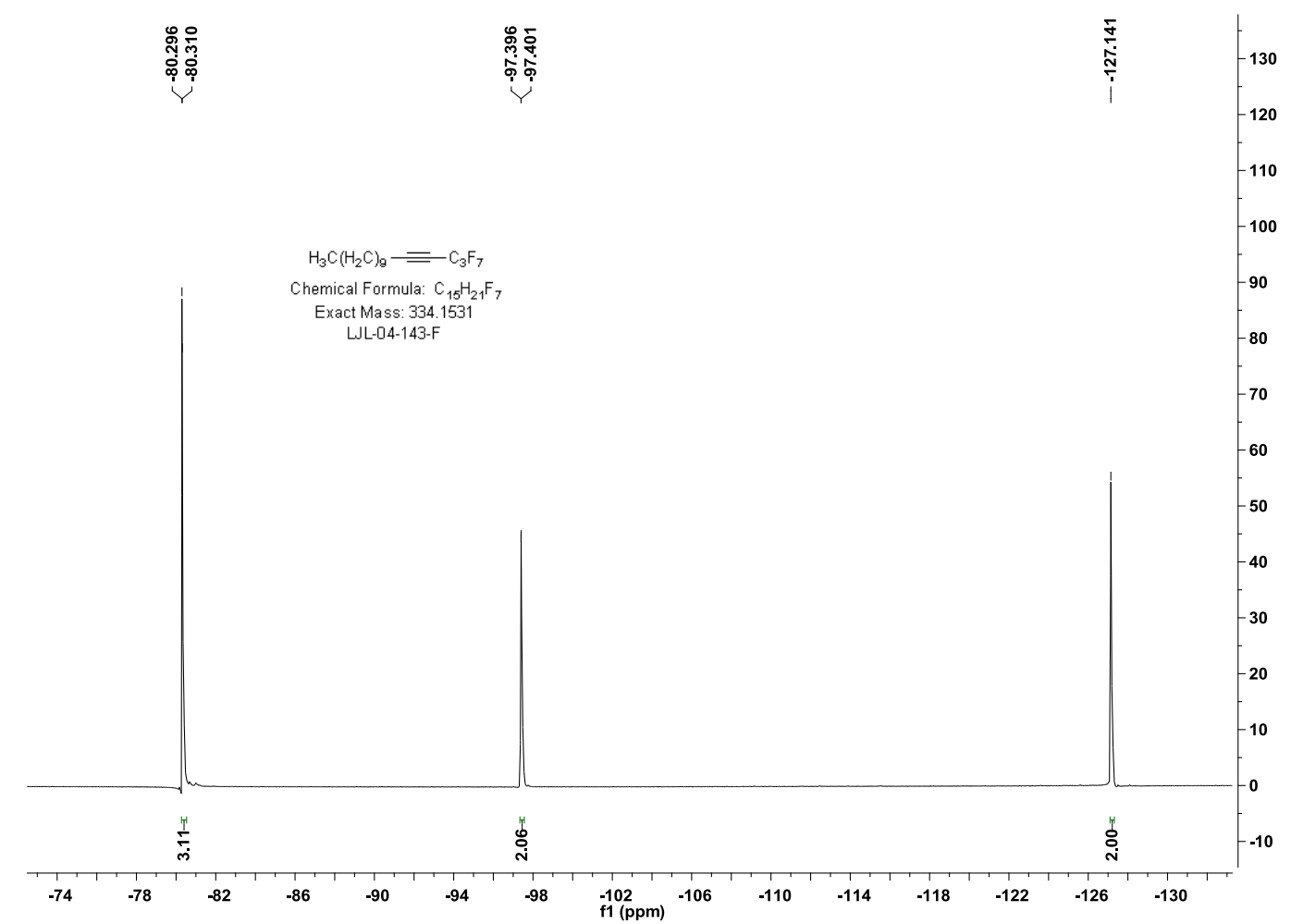




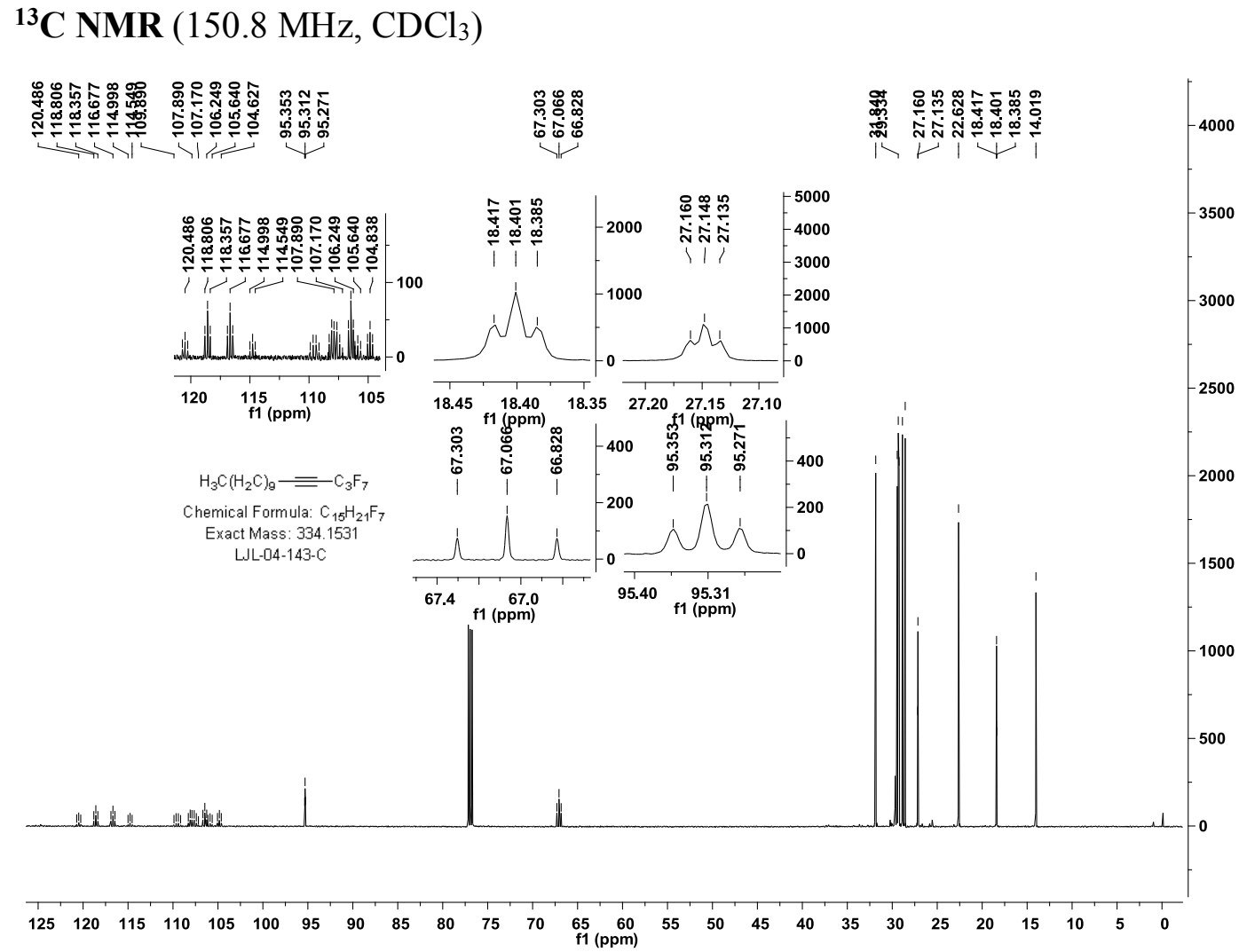

6,6,7,7,8,8,8-Heptafluorooct-4-yn-1-yl acetate (5n)

${ }^{1} \mathbf{H}$ NMR $\left(600 \mathrm{MHz}, \mathrm{CDCl}_{3}\right)$

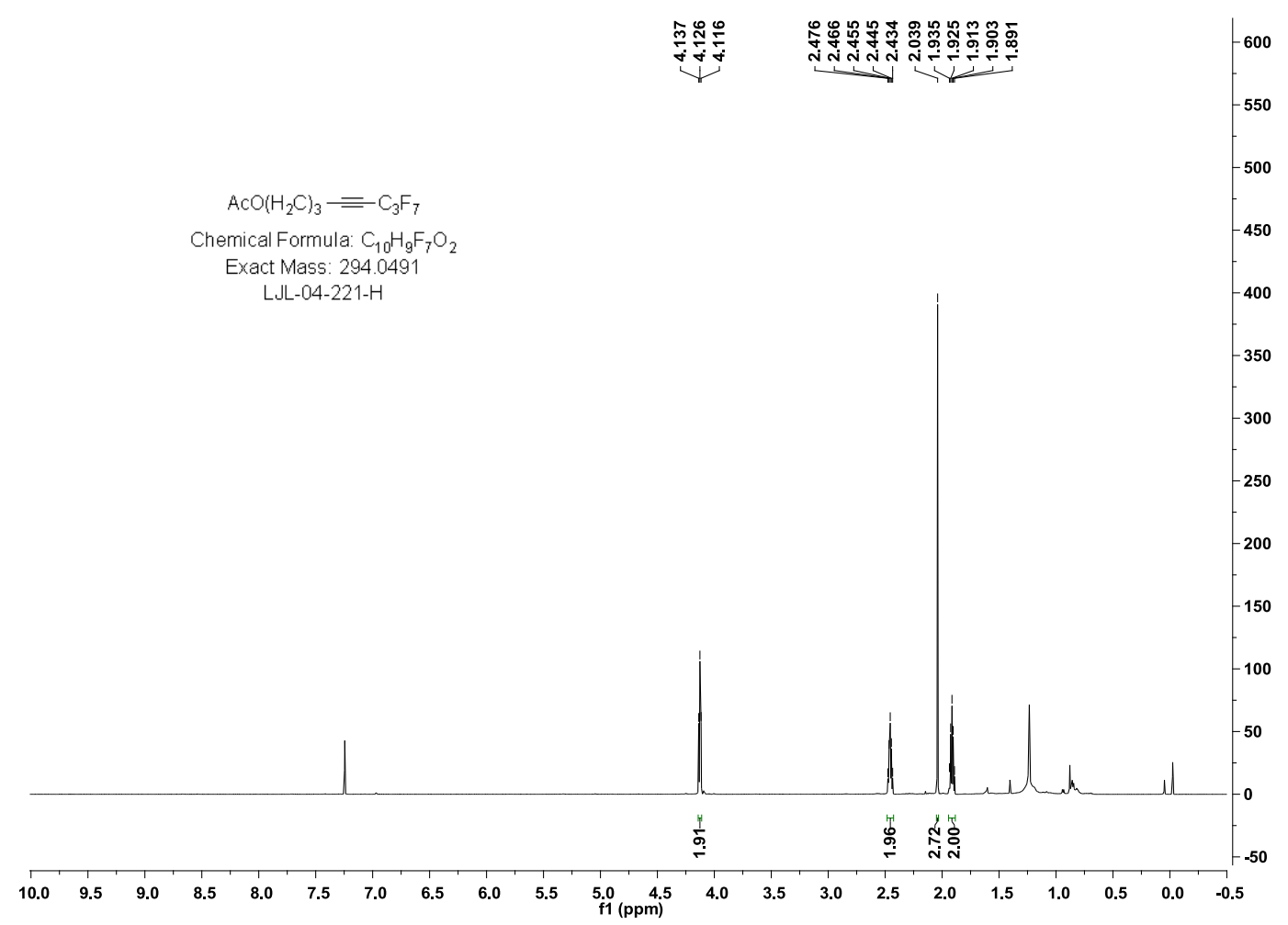


${ }^{19}$ F NMR $\left(564 \mathrm{MHz}, \mathrm{CDCl}_{3}\right)$

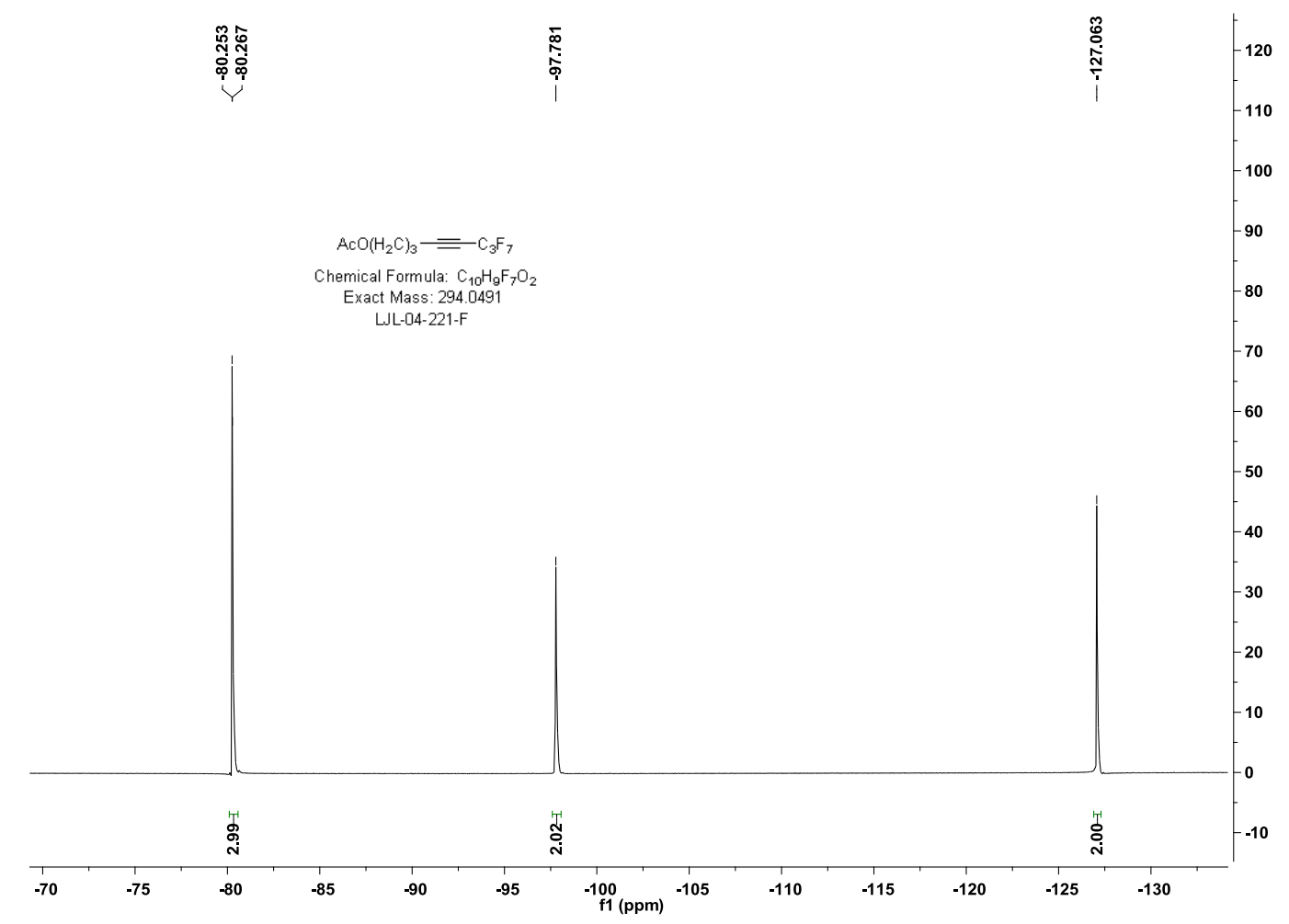

${ }^{13}$ C NMR (150.8 MHz, $\left.\mathrm{CDCl}_{3}\right)$

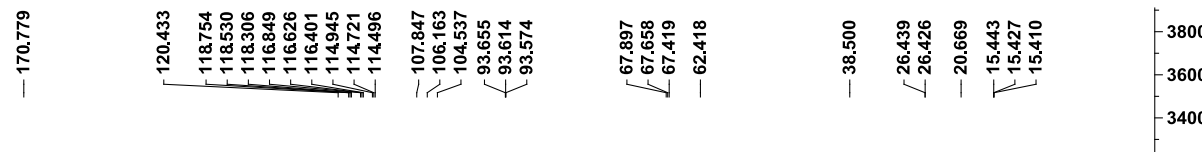

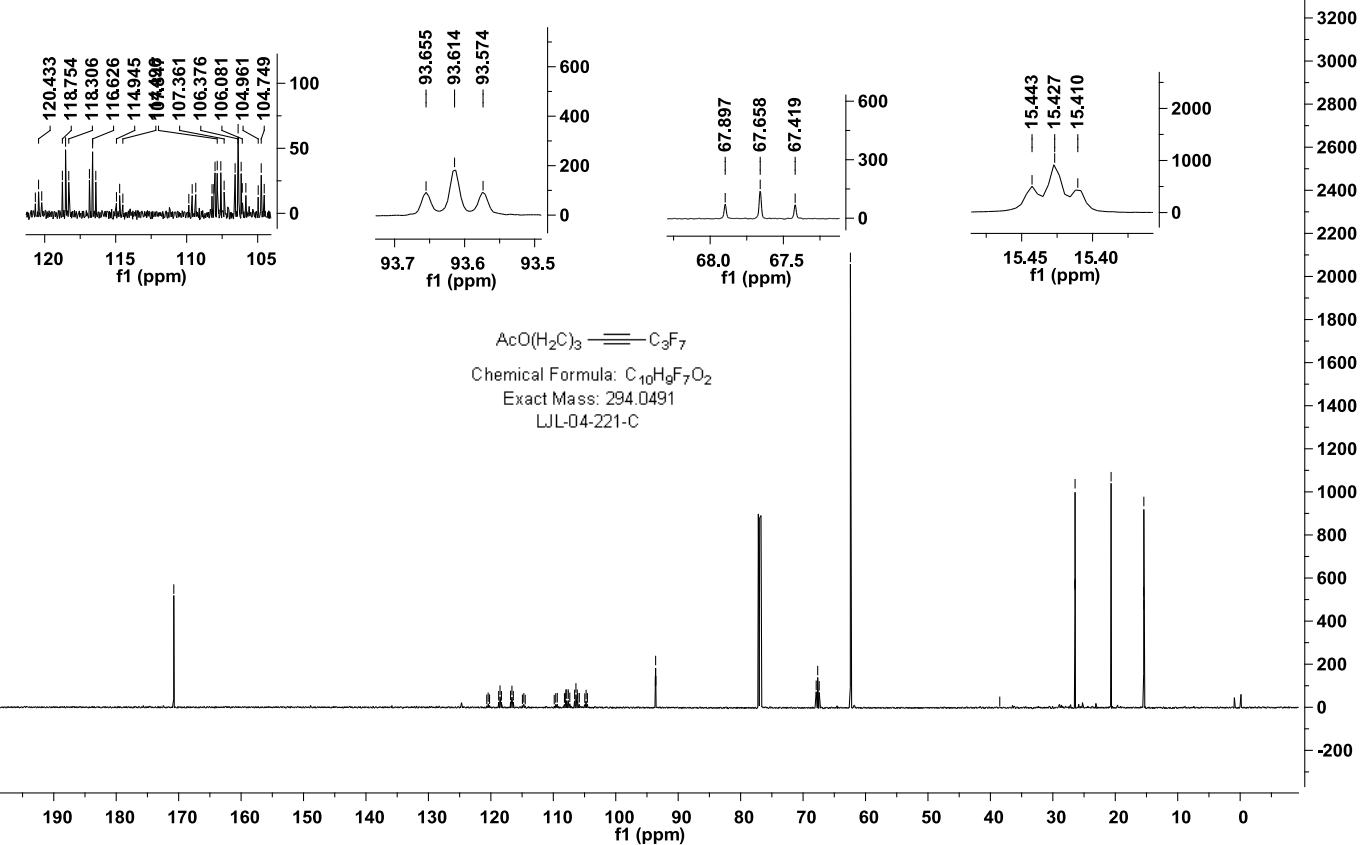


1-(perfluorooct-1-yn-1-yl)-4-propylbenzene (7a)

${ }^{1} \mathbf{H}$ NMR (600 MHz, $\left.\mathrm{CDCl}_{3}\right)$

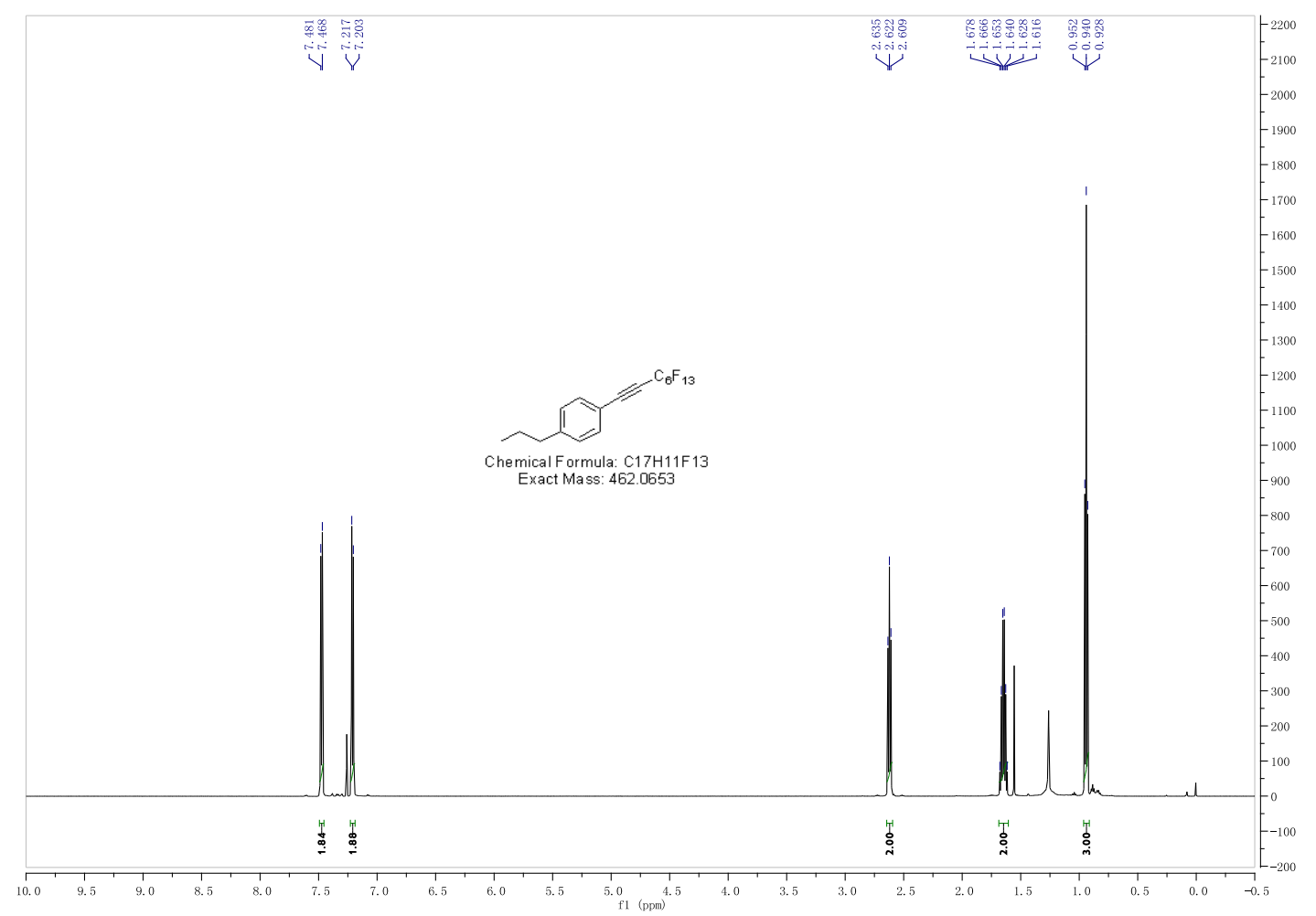

${ }^{19}$ F NMR (564 MHz, $\left.\mathrm{CDCl}_{3}\right)$

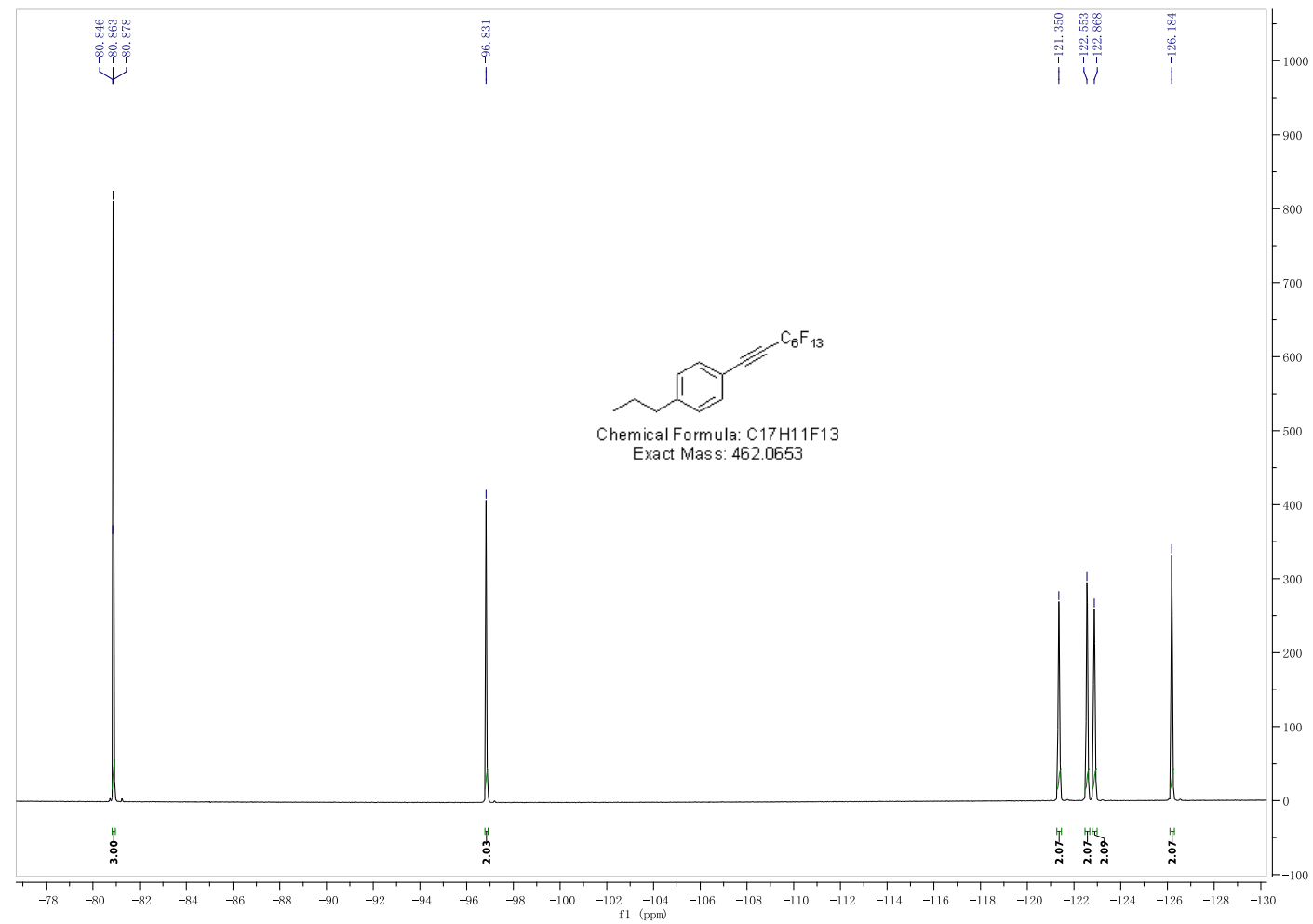


1-pentyl-4-(perfluorooct-1-yn-1-yl)benzene (7b)

${ }^{1}$ H NMR (600 MHz, $\left.\mathrm{CDCl}_{3}\right)$

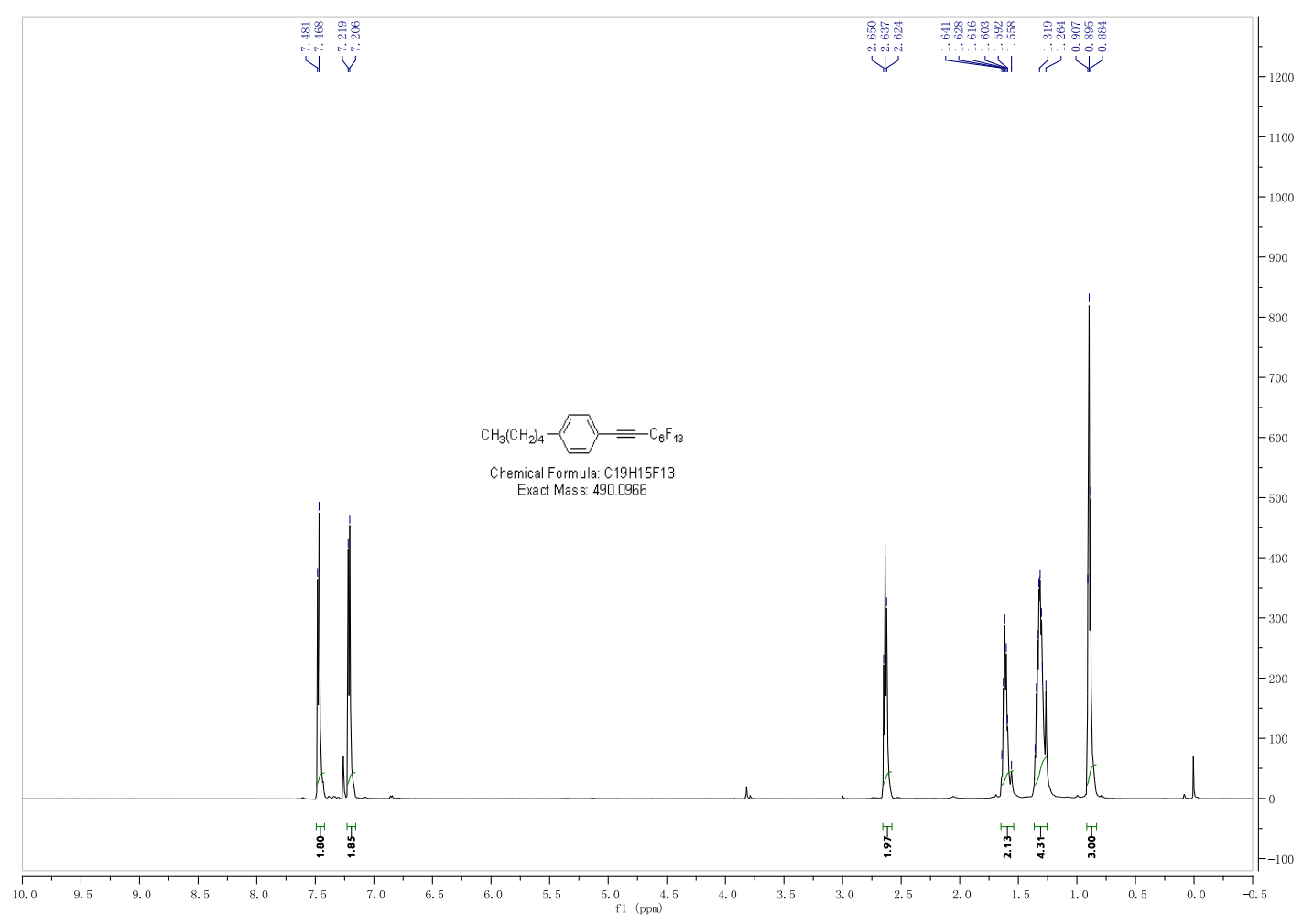

${ }^{19}$ F NMR (564 MHz, $\left.\mathrm{CDCl}_{3}\right)$

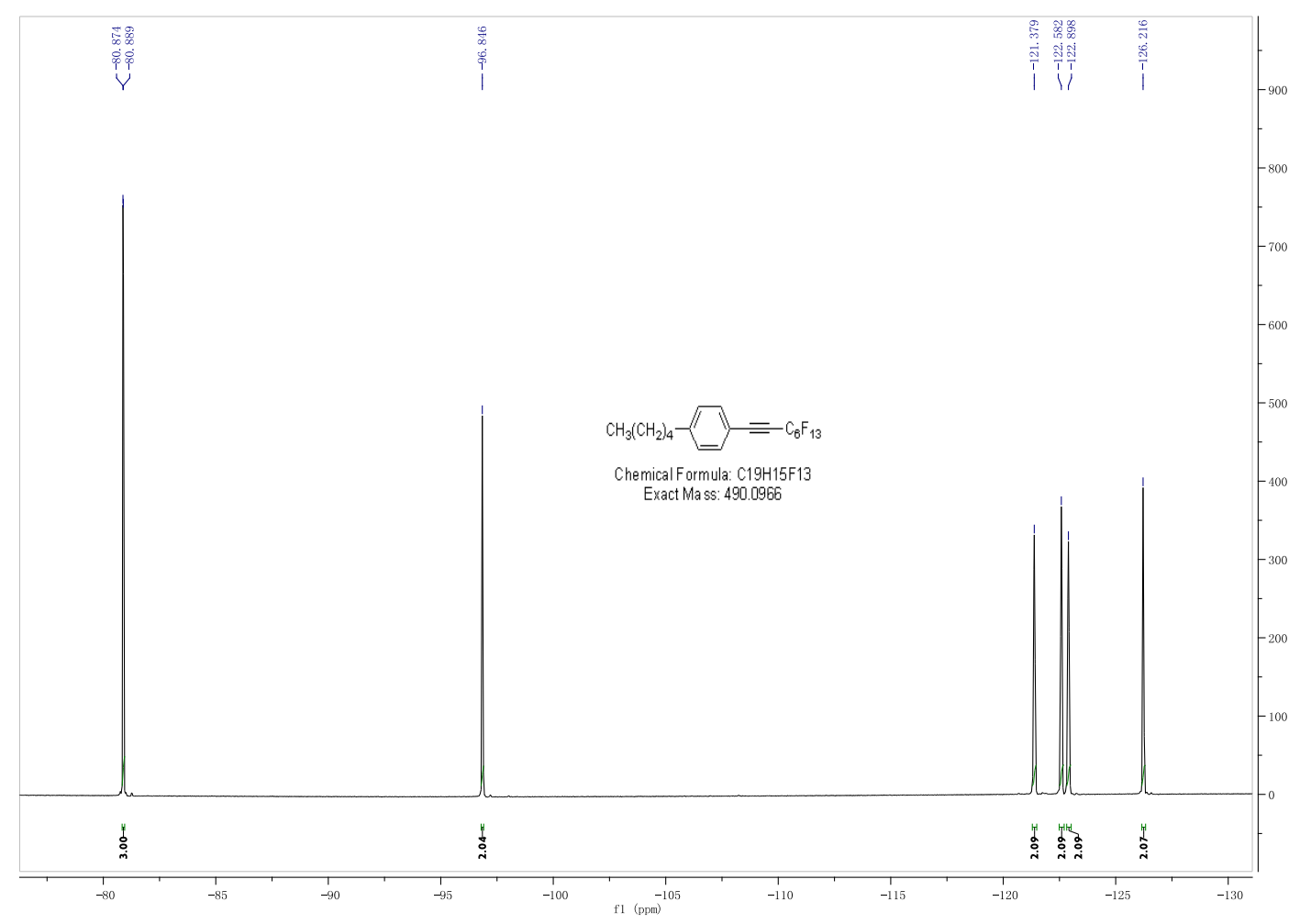


(perfluorooct-1-yn-1-yl)benzene (7c)

${ }^{1} \mathbf{H}$ NMR (600 MHz, $\left.\mathrm{CDCl}_{3}\right)$

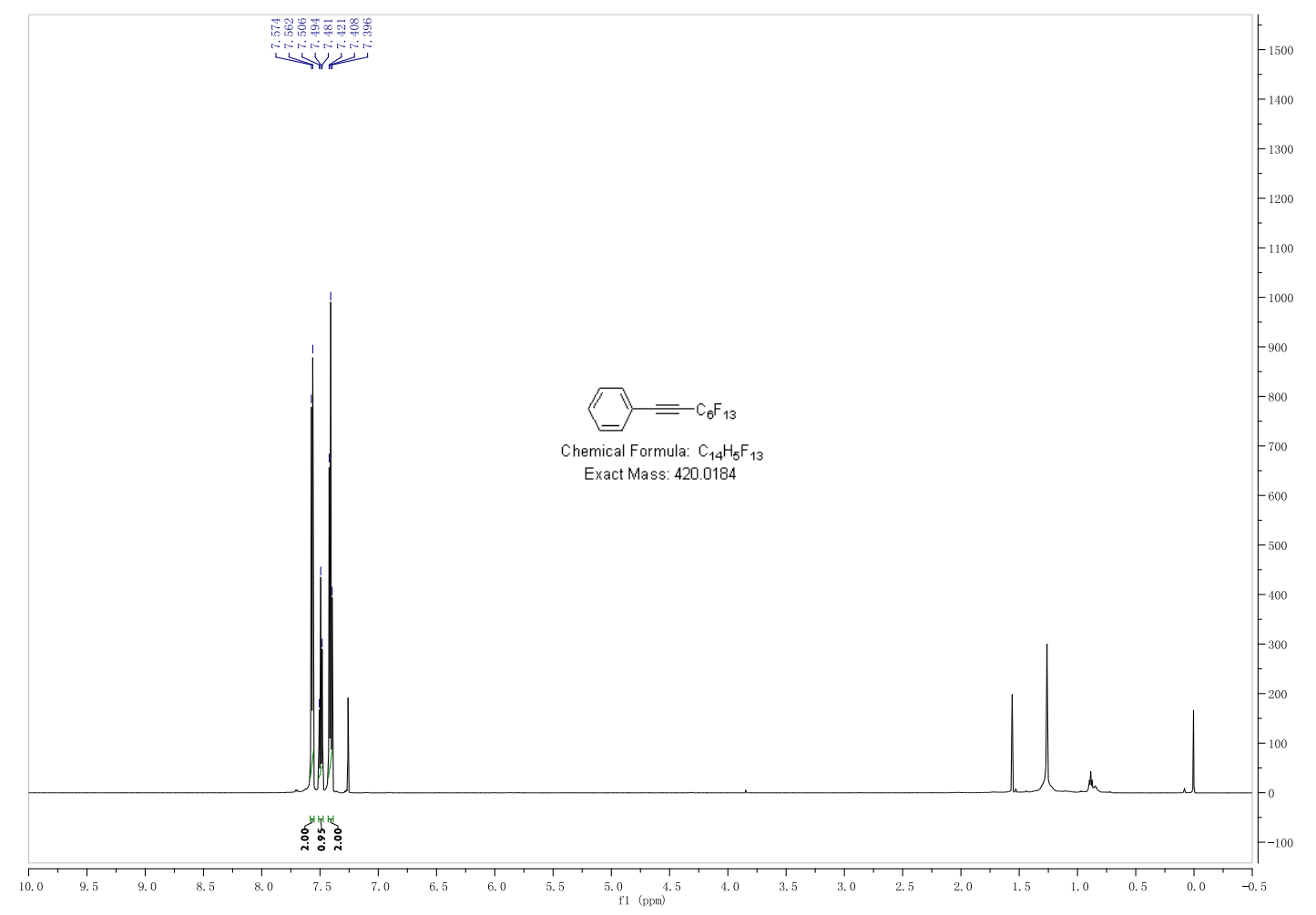

${ }^{19}$ F NMR (564 MHz, $\left.\mathrm{CDCl}_{3}\right)$

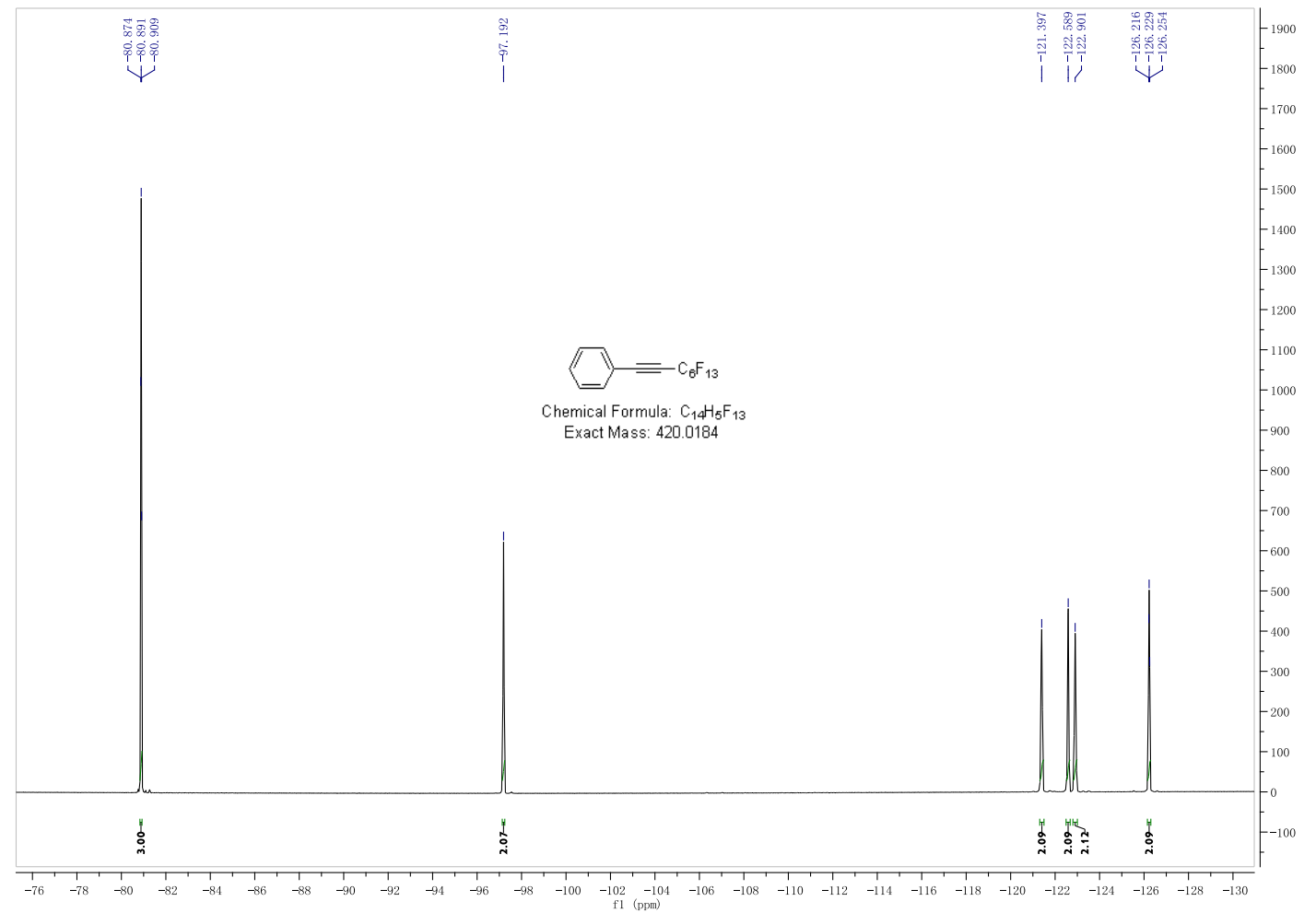


1-methoxy-4-(perfluorooct-1-yn-1-yl)benzene (7d)

${ }^{1} \mathbf{H}$ NMR (600 MHz, $\left.\mathrm{CDCl}_{3}\right)$

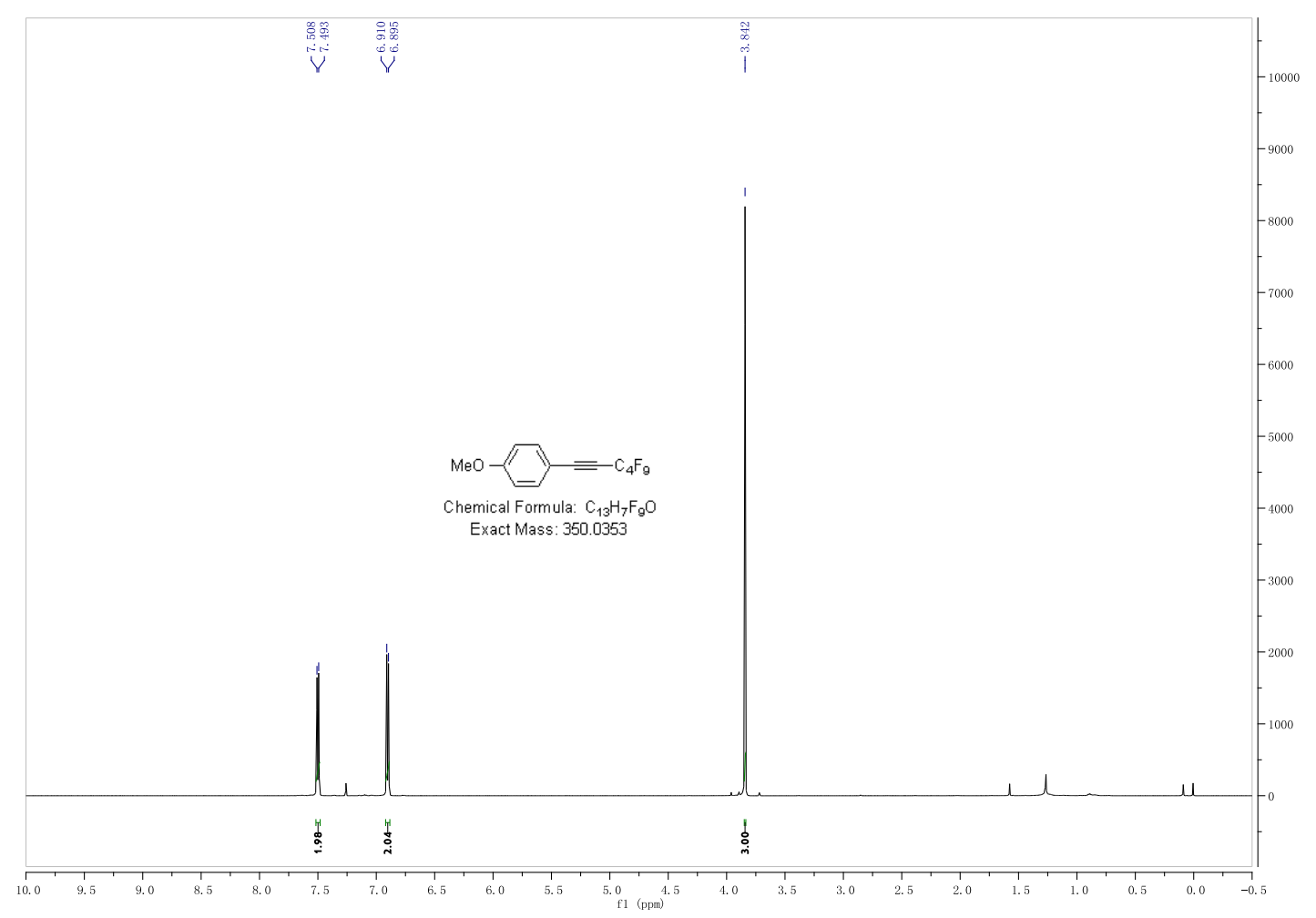

${ }^{19}$ F NMR (564 MHz, $\left.\mathrm{CDCl}_{3}\right)$

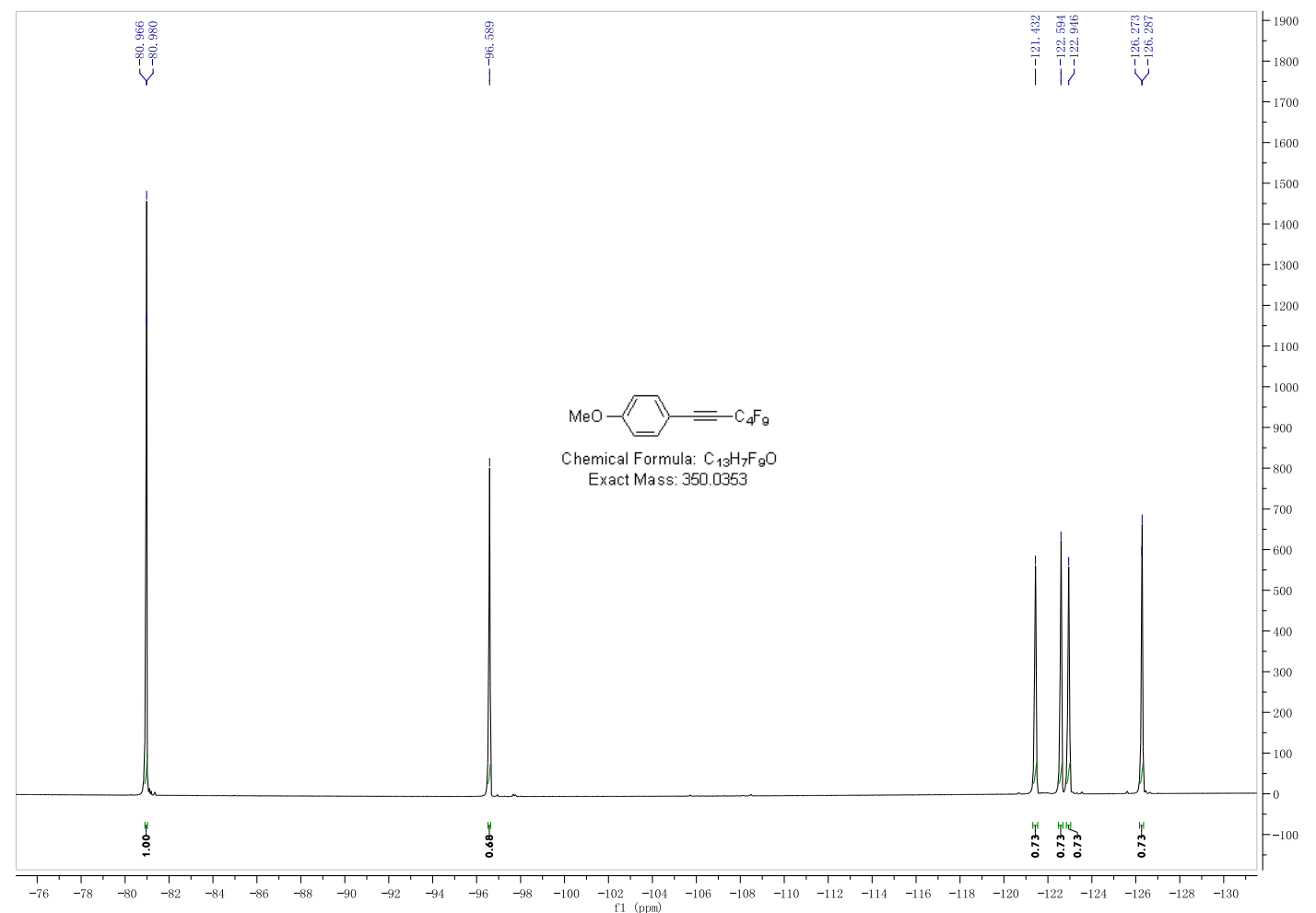


1-chloro-4-(perfluorooct-1-yn-1-yl)benzene (7h)

${ }^{1} \mathbf{H}$ NMR (600 MHz, $\left.\mathrm{CDCl}_{3}\right)$

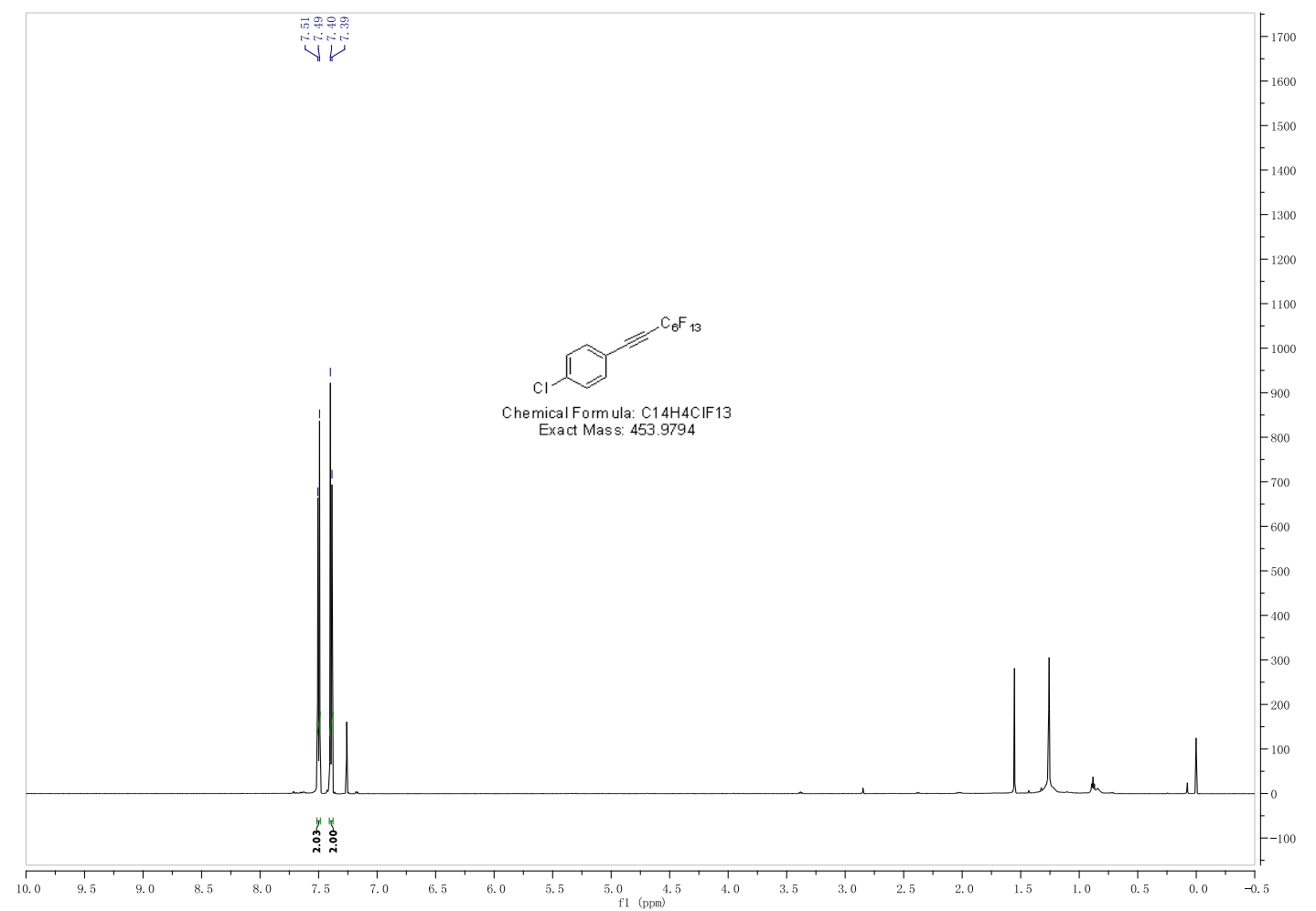

${ }^{19}$ F NMR (564 MHz, $\left.\mathrm{CDCl}_{3}\right)$

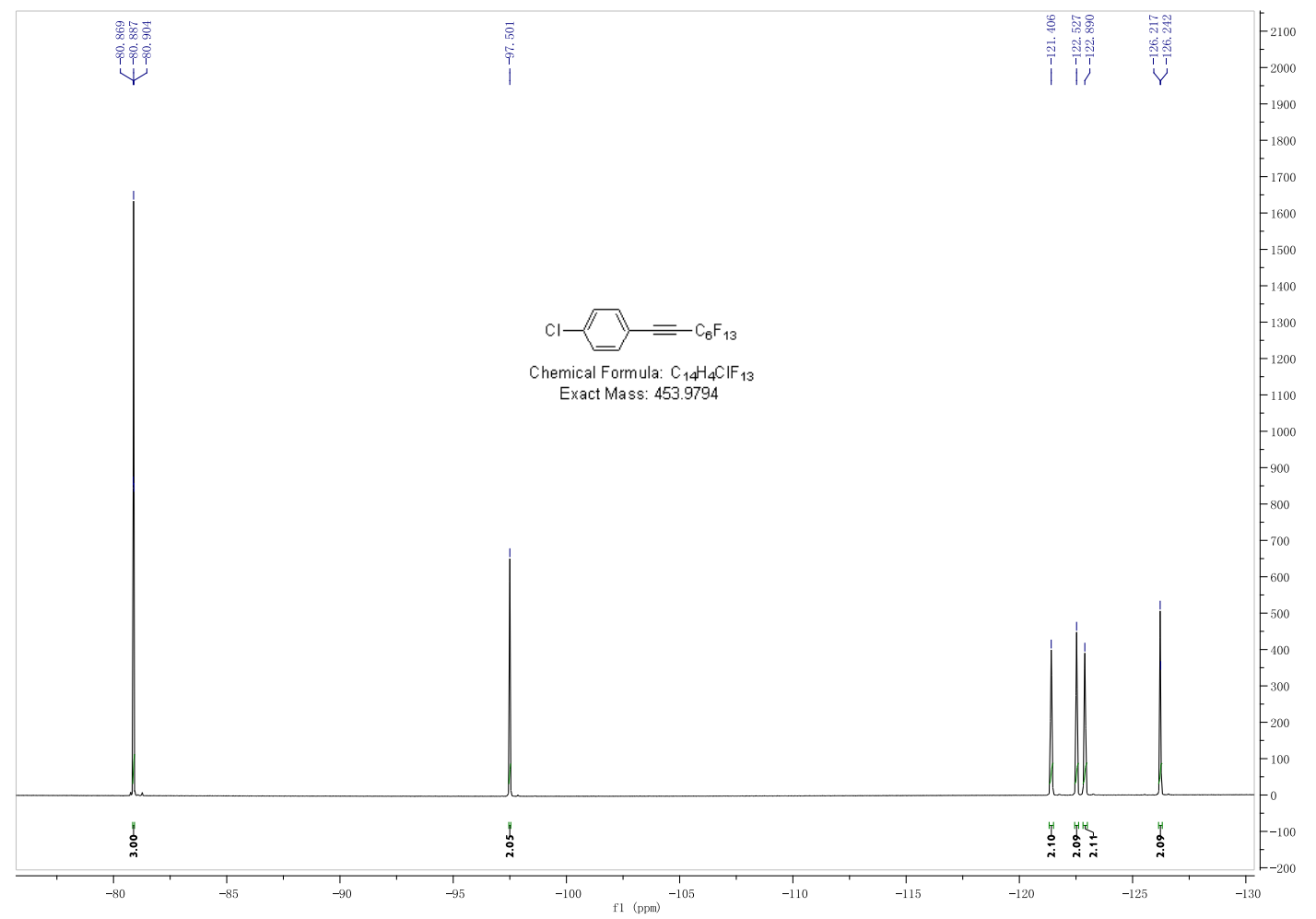


Methyl 4-(perfluorooct-1-yn-1-yl)benzoate (7i)

${ }^{1} \mathbf{H}$ NMR (600 MHz, $\left.\mathrm{CDCl}_{3}\right)$

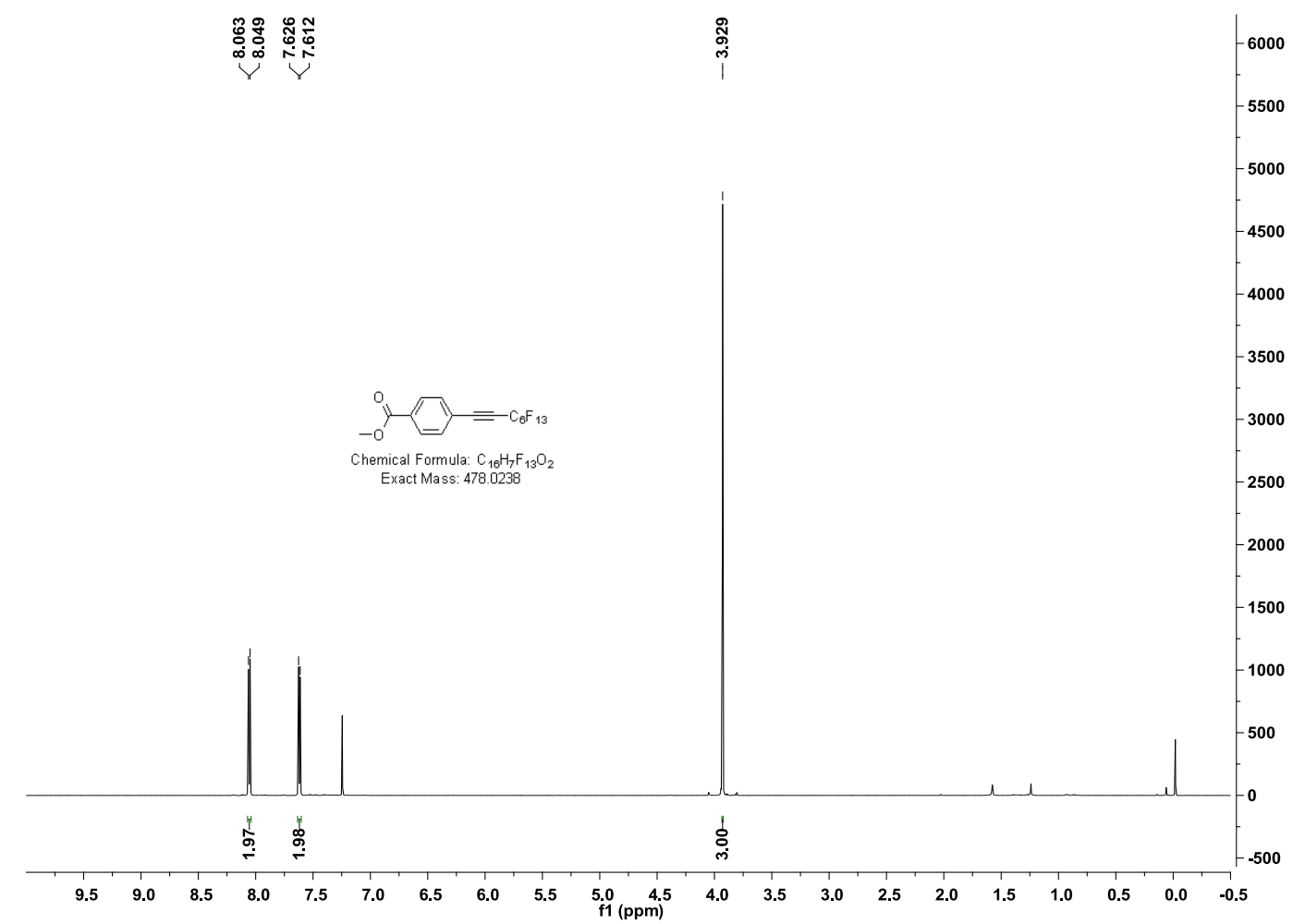

${ }^{19}$ F NMR (564 MHz, $\left.\mathrm{CDCl}_{3}\right)$

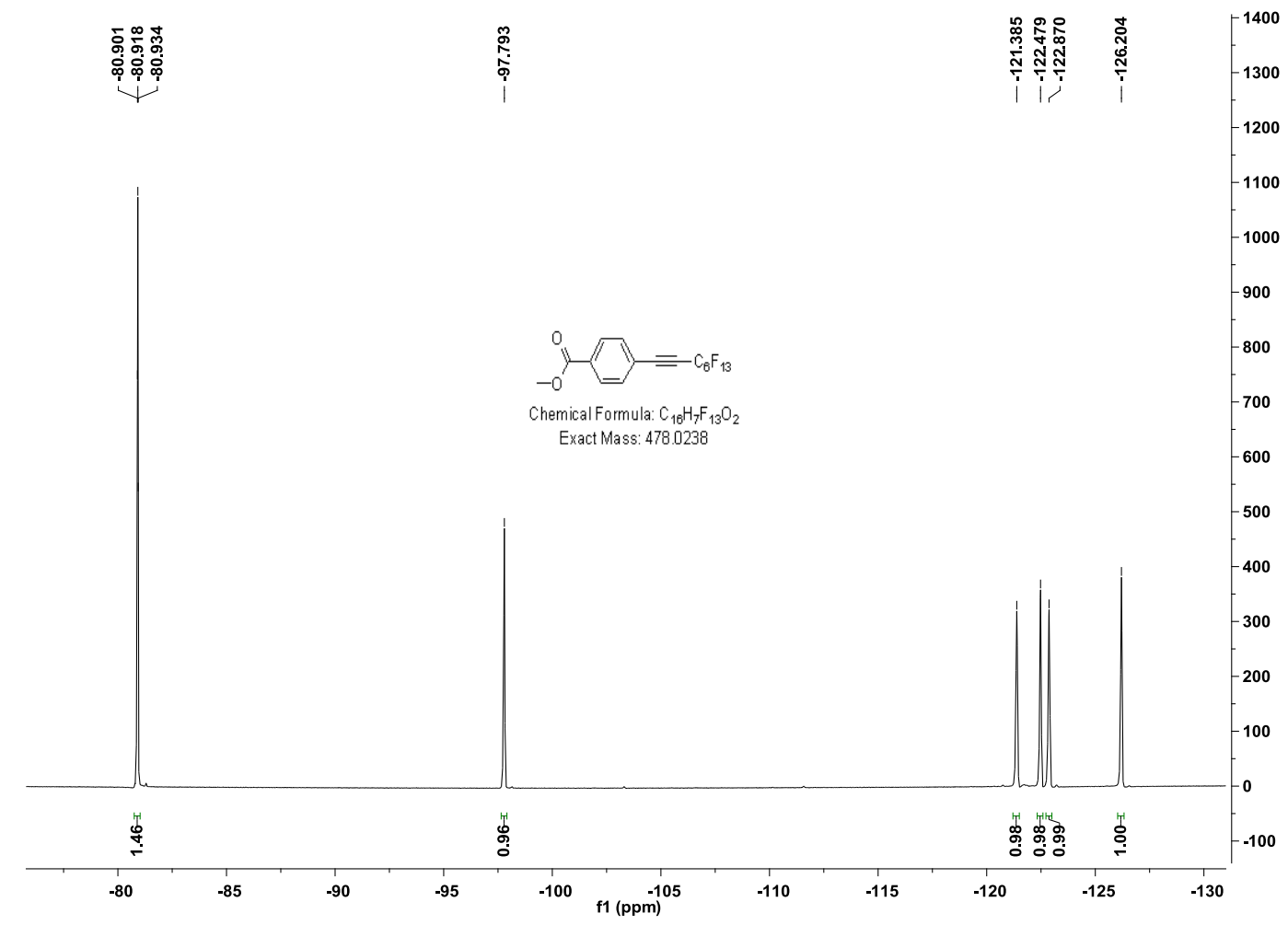


${ }^{13} \mathbf{C ~ N M R}\left(150.8 \mathrm{MHz}, \mathrm{CDCl}_{3}\right)$

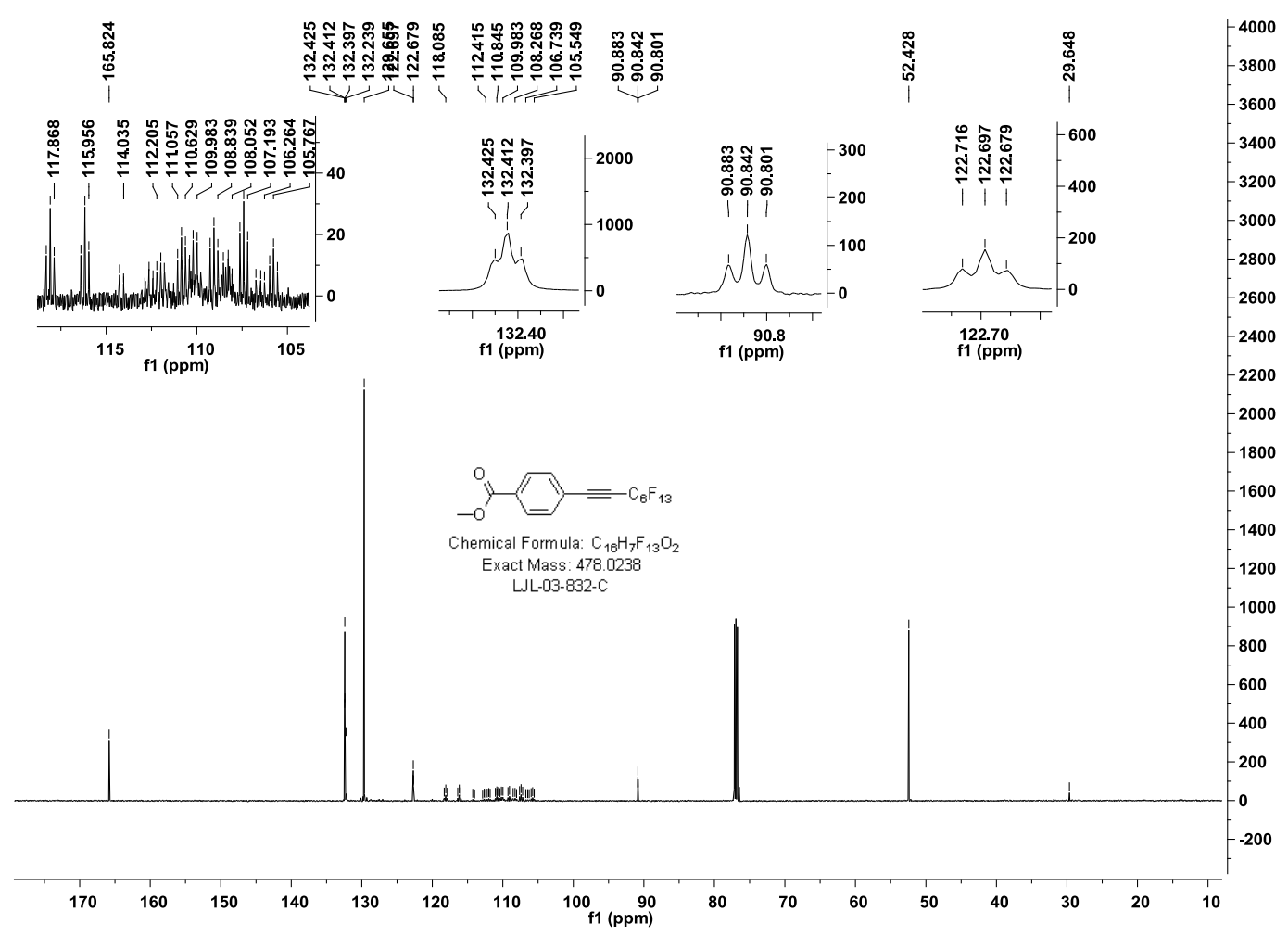

3-(Perfluorooct-1-yn-1-yl)thiophene (7j)

${ }^{1} \mathbf{H}$ NMR $\left(600 \mathrm{MHz}, \mathrm{CDCl}_{3}\right)$

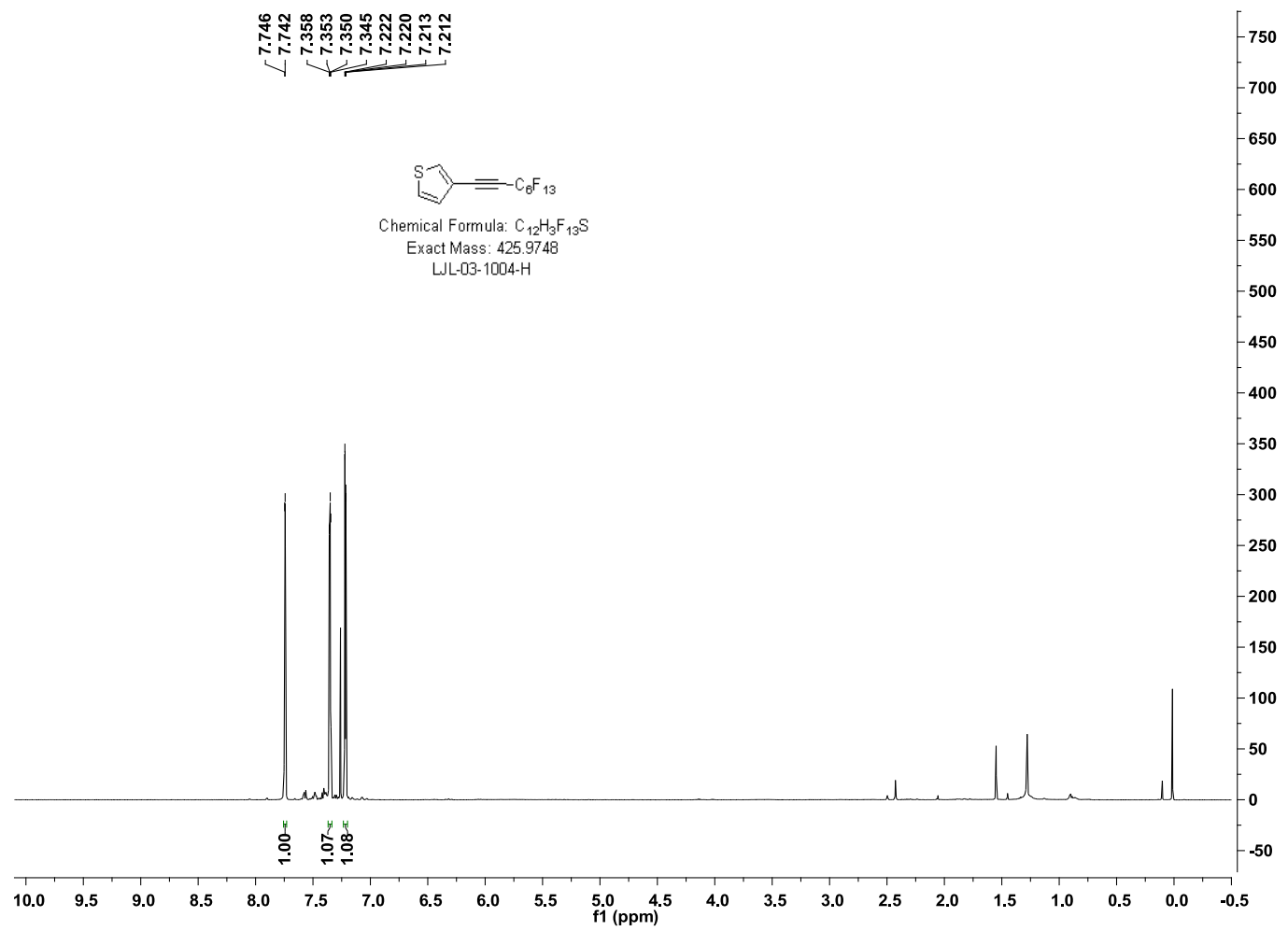


${ }^{19}$ F NMR $\left(564 \mathrm{MHz}, \mathrm{CDCl}_{3}\right)$

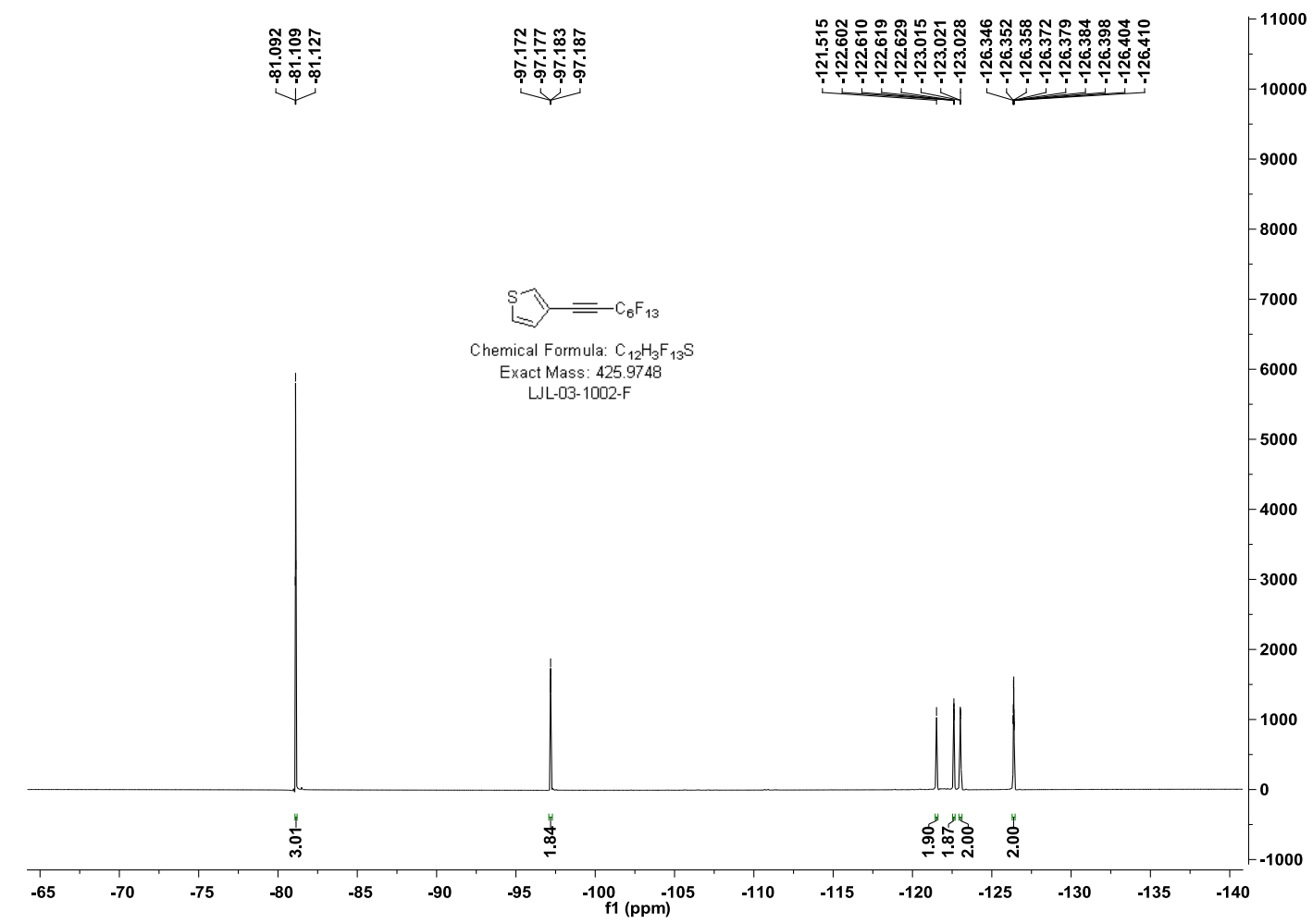

${ }^{13}$ C NMR (150.8 MHz, $\left.\mathrm{CDCl}_{3}\right)$

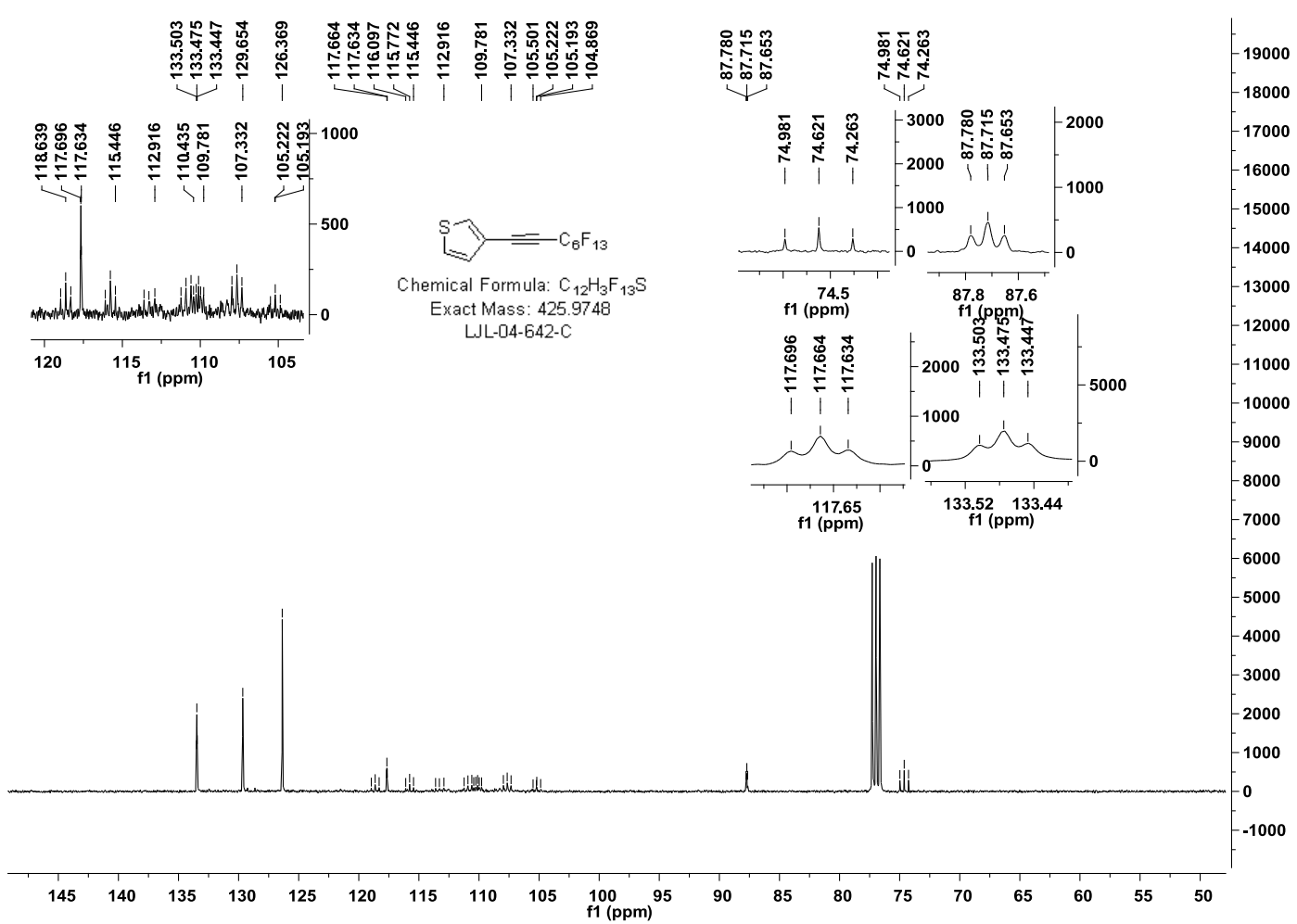


3-(Perfluorohex-1-yn-1-yl)pyridine (7k)

${ }^{1} \mathbf{H}$ NMR (600 MHz, $\left.\mathrm{CDCl}_{3}\right)$

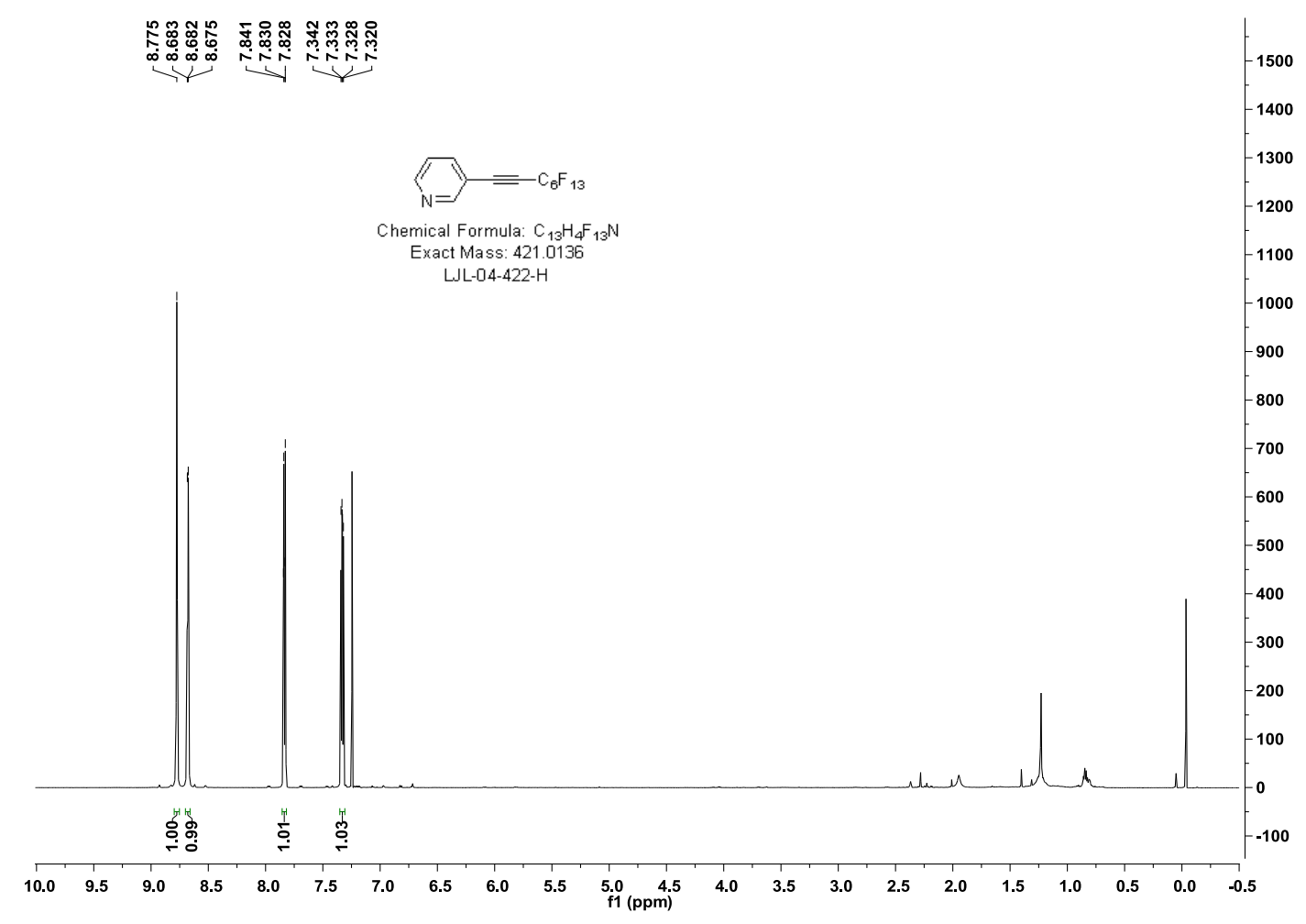

${ }^{19}$ F NMR $\left(564 \mathrm{MHz}, \mathrm{CDCl}_{3}\right)$

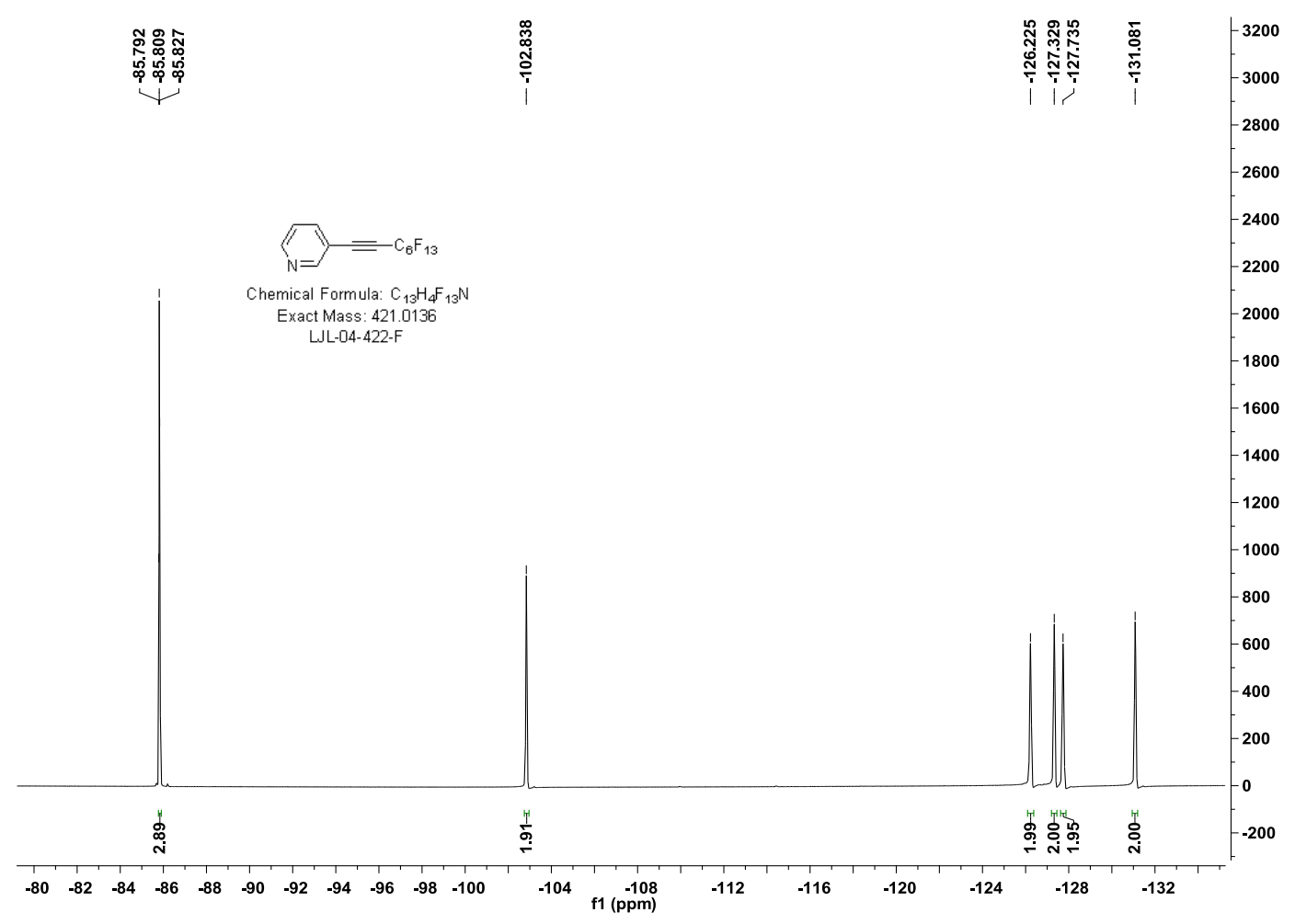




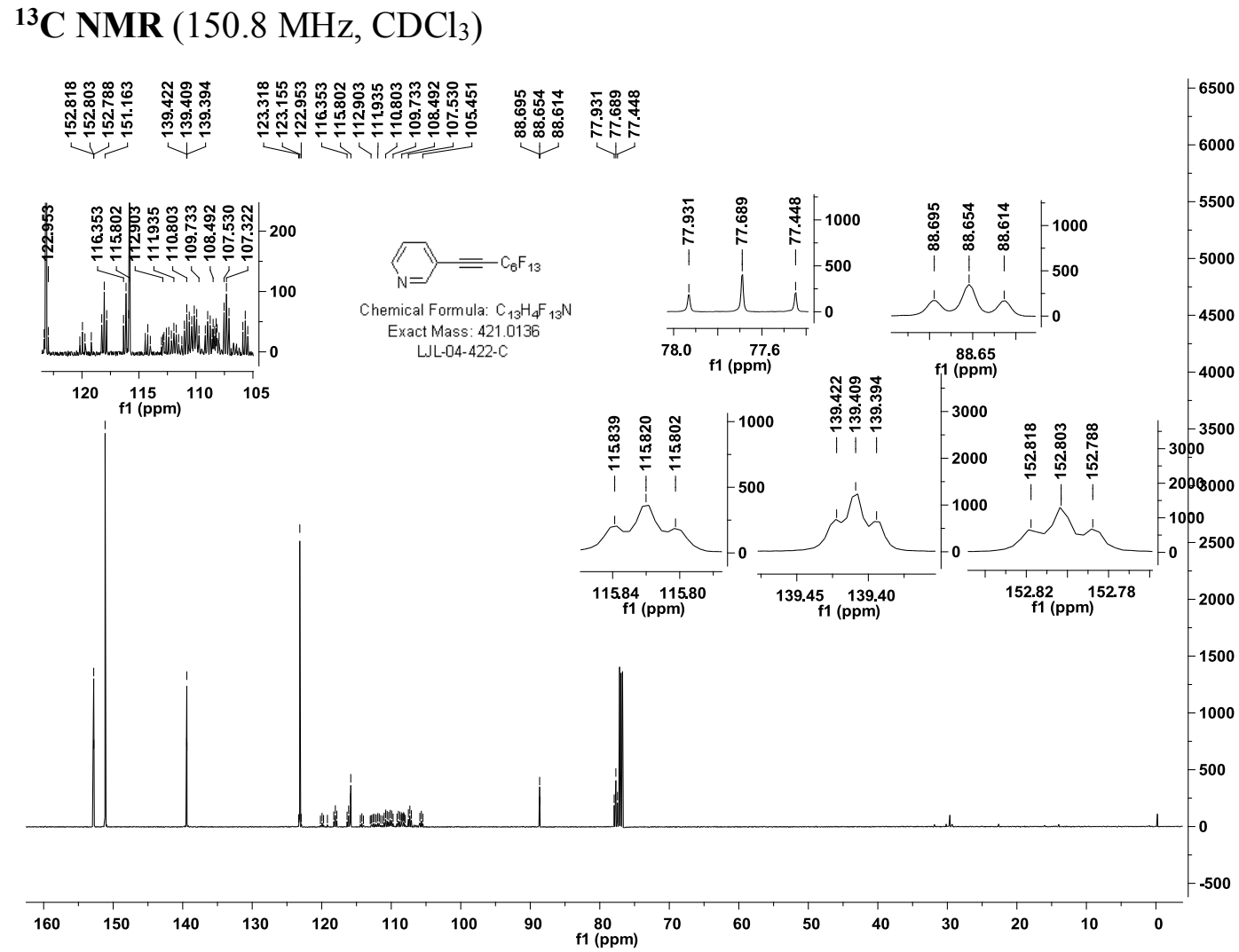

\section{1,1,1,2,2,3,3,4,4,5,5,6,6-Tridecafluorooctadec-7-yne (7l)}

${ }^{1} \mathbf{H}$ NMR $\left(600 \mathrm{MHz}, \mathrm{CDCl}_{3}\right)$

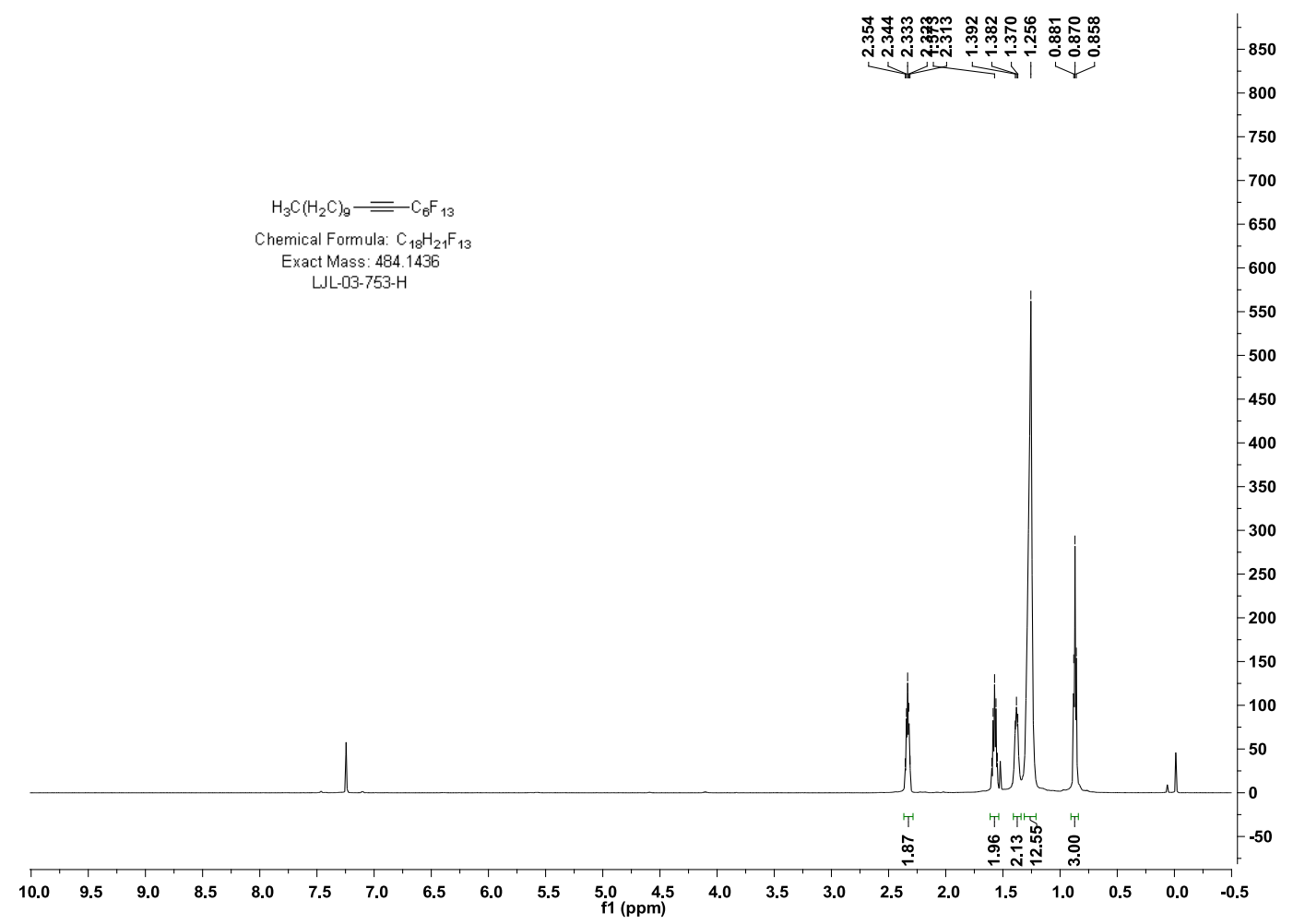


${ }^{19}$ F NMR $\left(564 \mathrm{MHz}, \mathrm{CDCl}_{3}\right)$

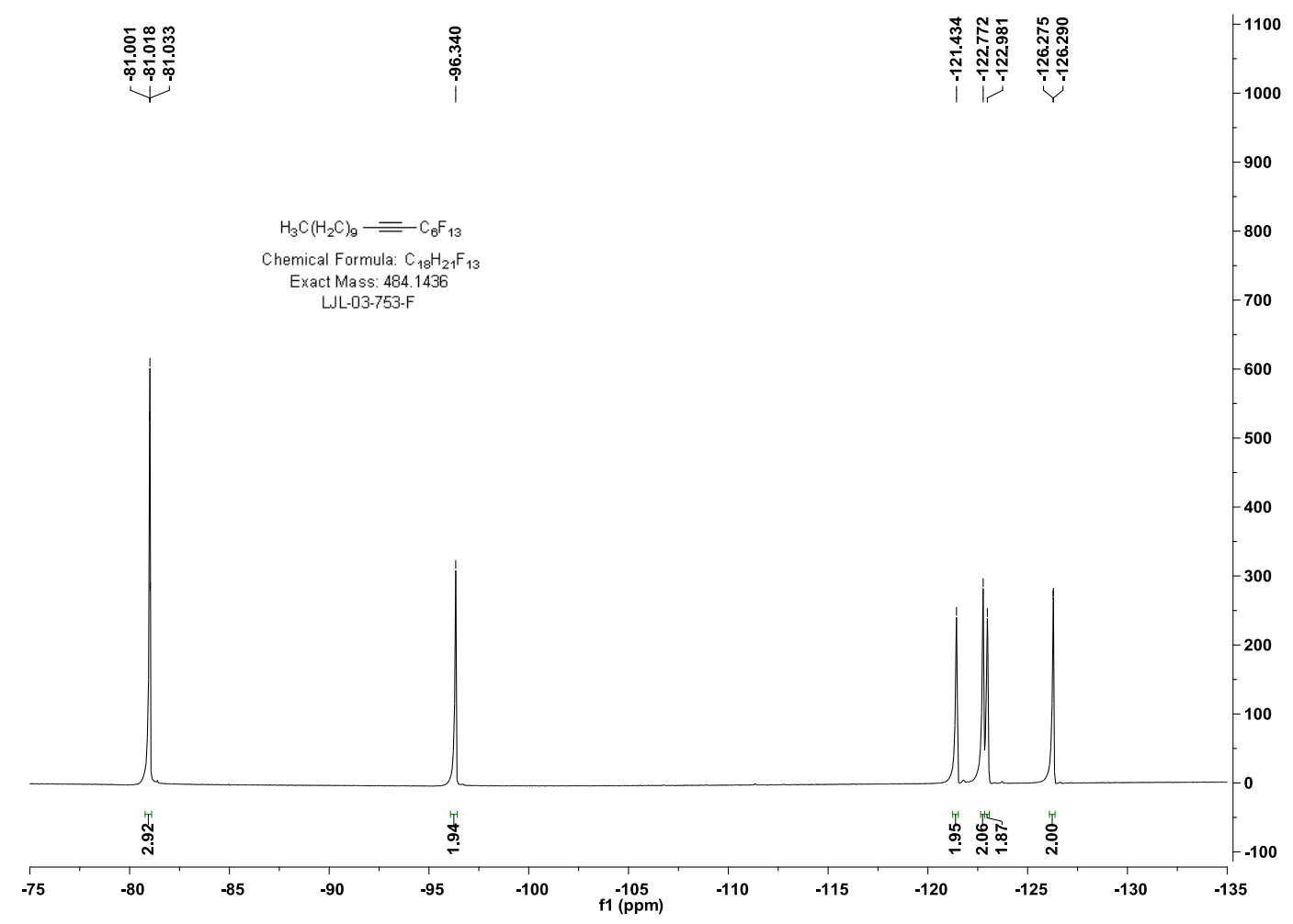

${ }^{13}$ C NMR (150.8 MHz, $\left.\mathrm{CDCl}_{3}\right)$

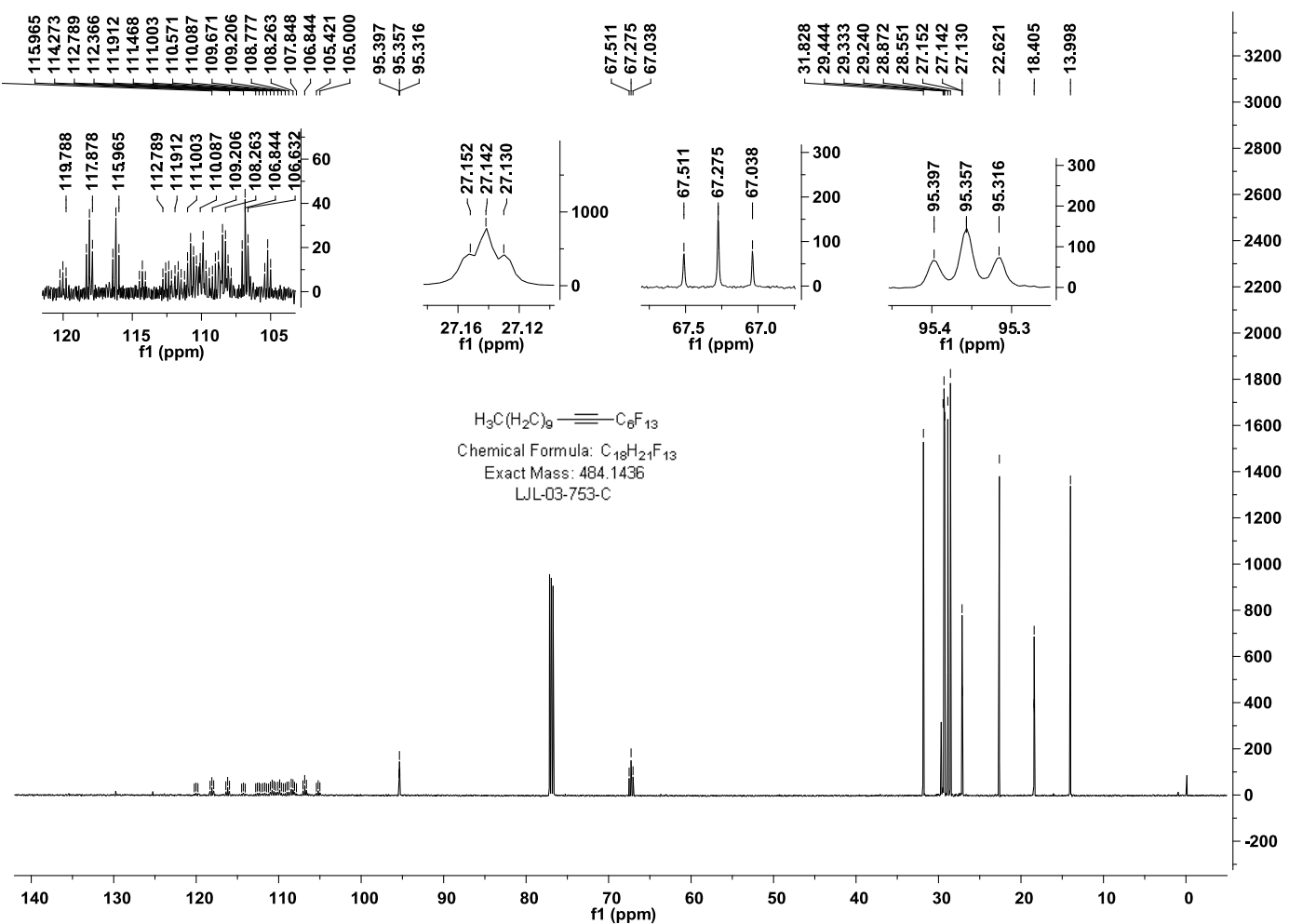




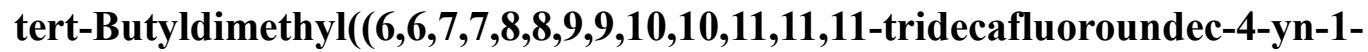

yl)oxy)silane (7 m)

${ }^{1} \mathbf{H}$ NMR $\left(600 \mathrm{MHz}, \mathrm{CDCl}_{3}\right)$

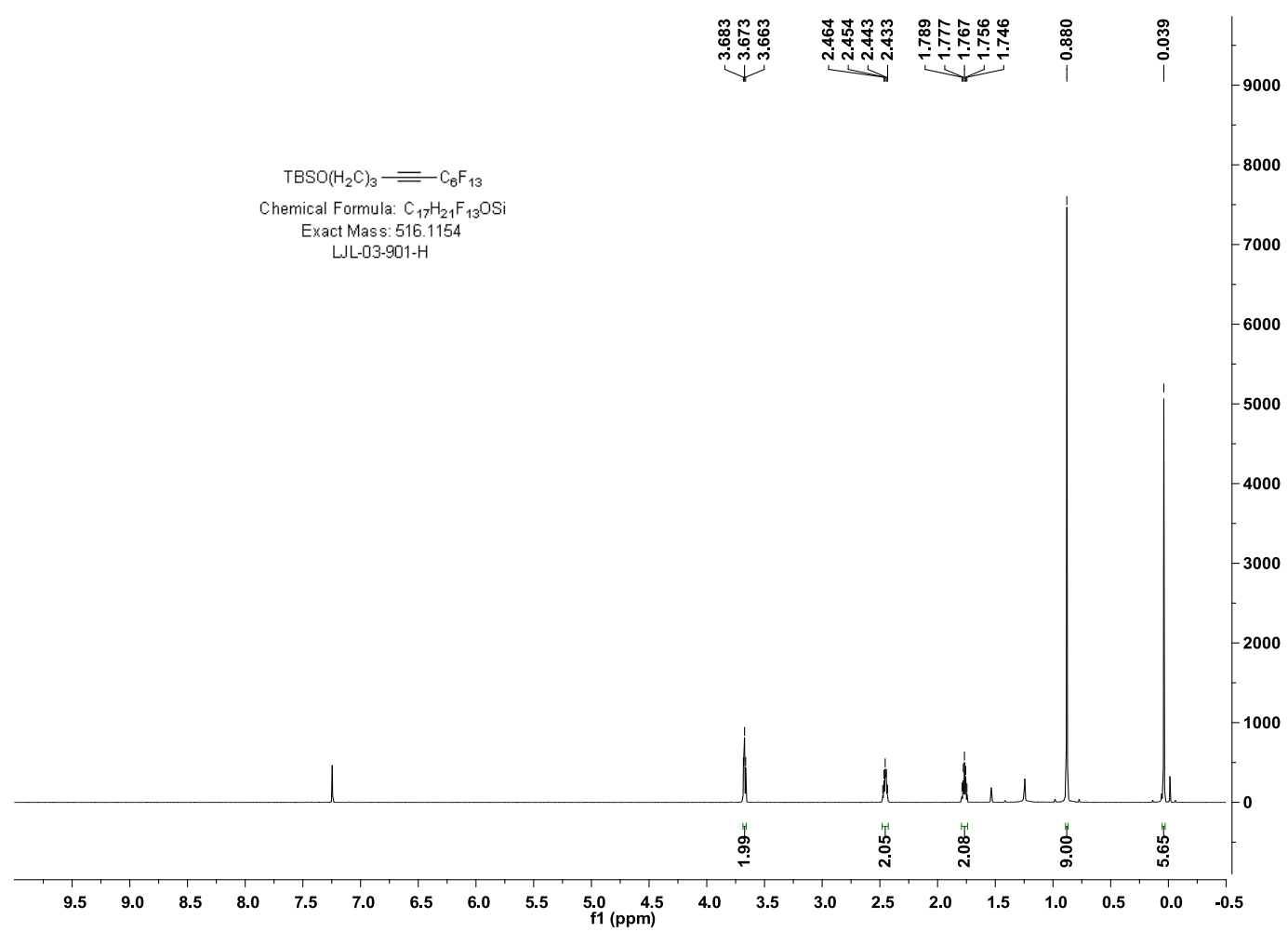

${ }^{19}$ F NMR $\left(564 \mathrm{MHz}, \mathrm{CDCl}_{3}\right)$

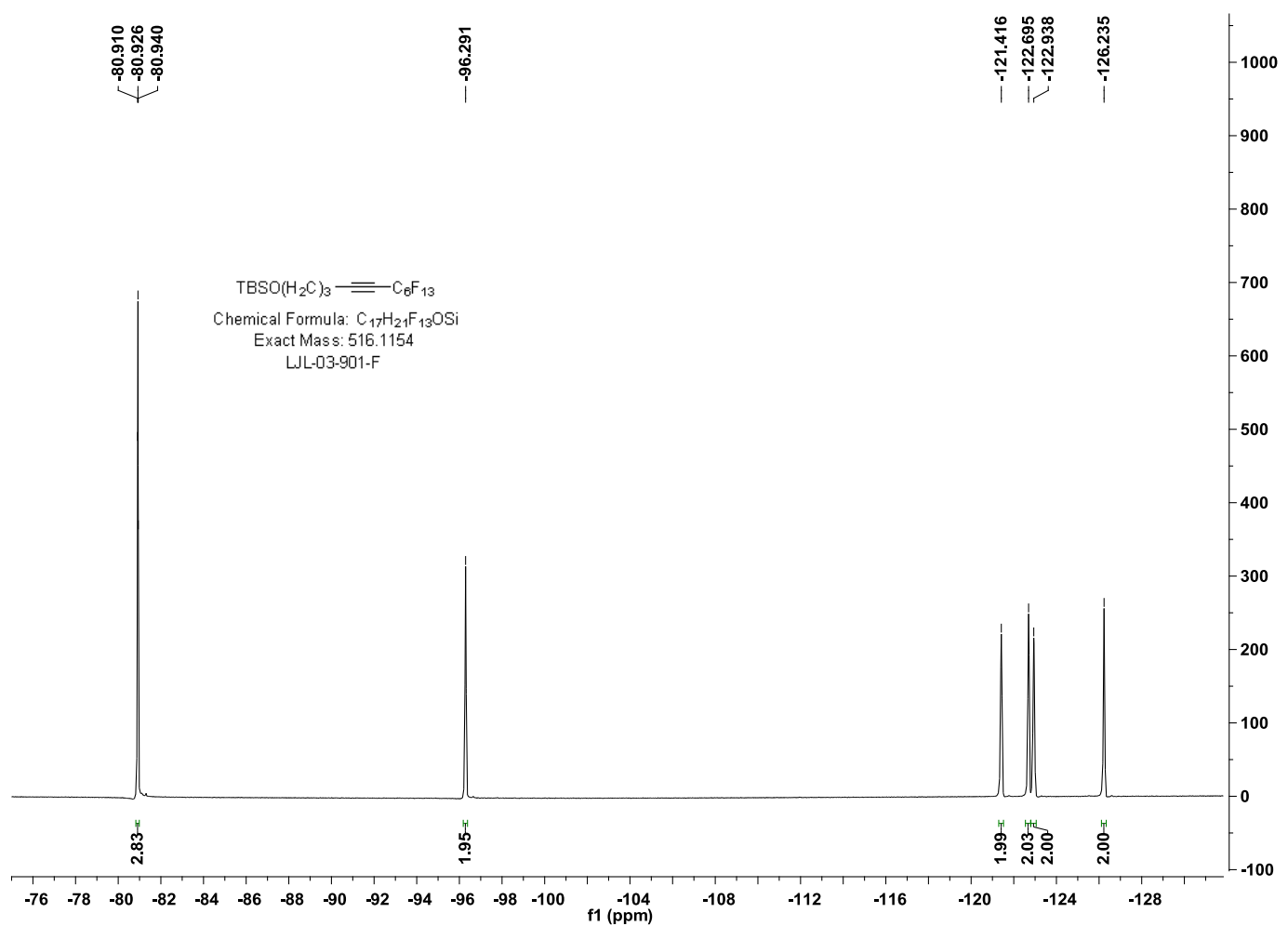




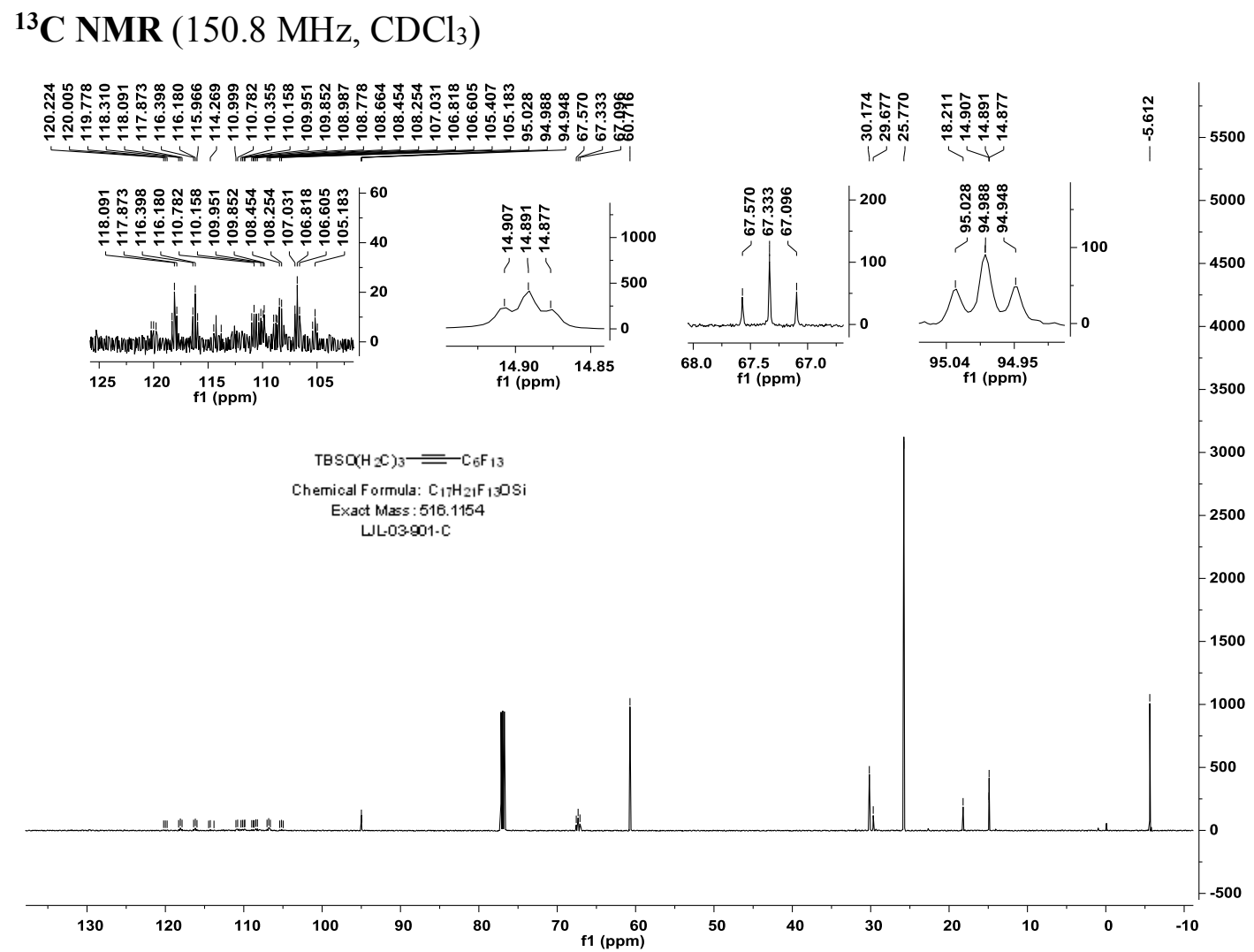

Diethyl (6,6,7,7,8,8,9,9,10,10,11,11,11-tridecafluoroundec-4-yn-1-yl) phosphate (7q) ${ }^{1} \mathbf{H}$ NMR $\left(600 \mathrm{MHz}, \mathrm{CDCl}_{3}\right)$

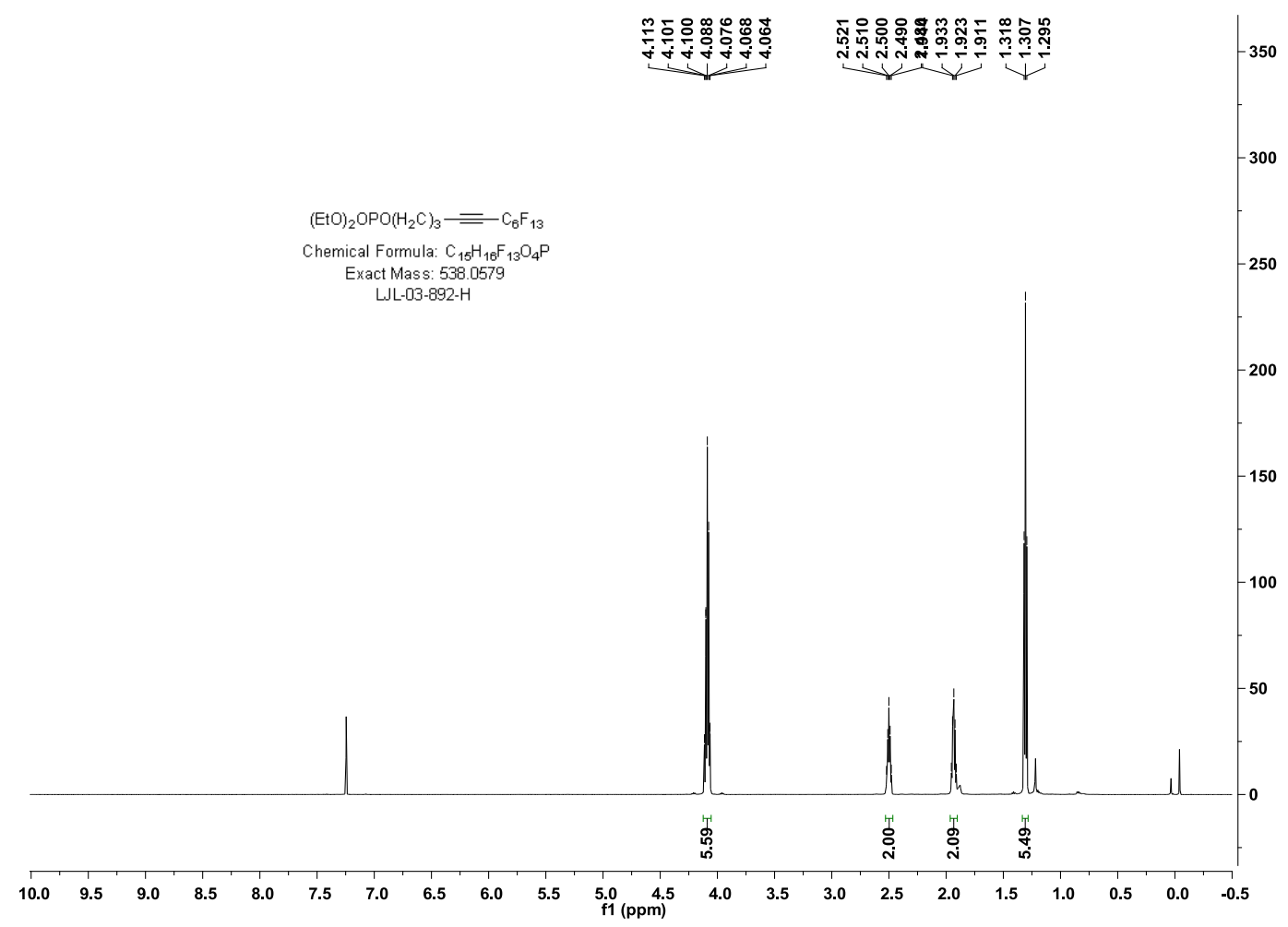


${ }^{19}$ F NMR $\left(150.8 \mathrm{MHz}, \mathrm{CDCl}_{3}\right)$

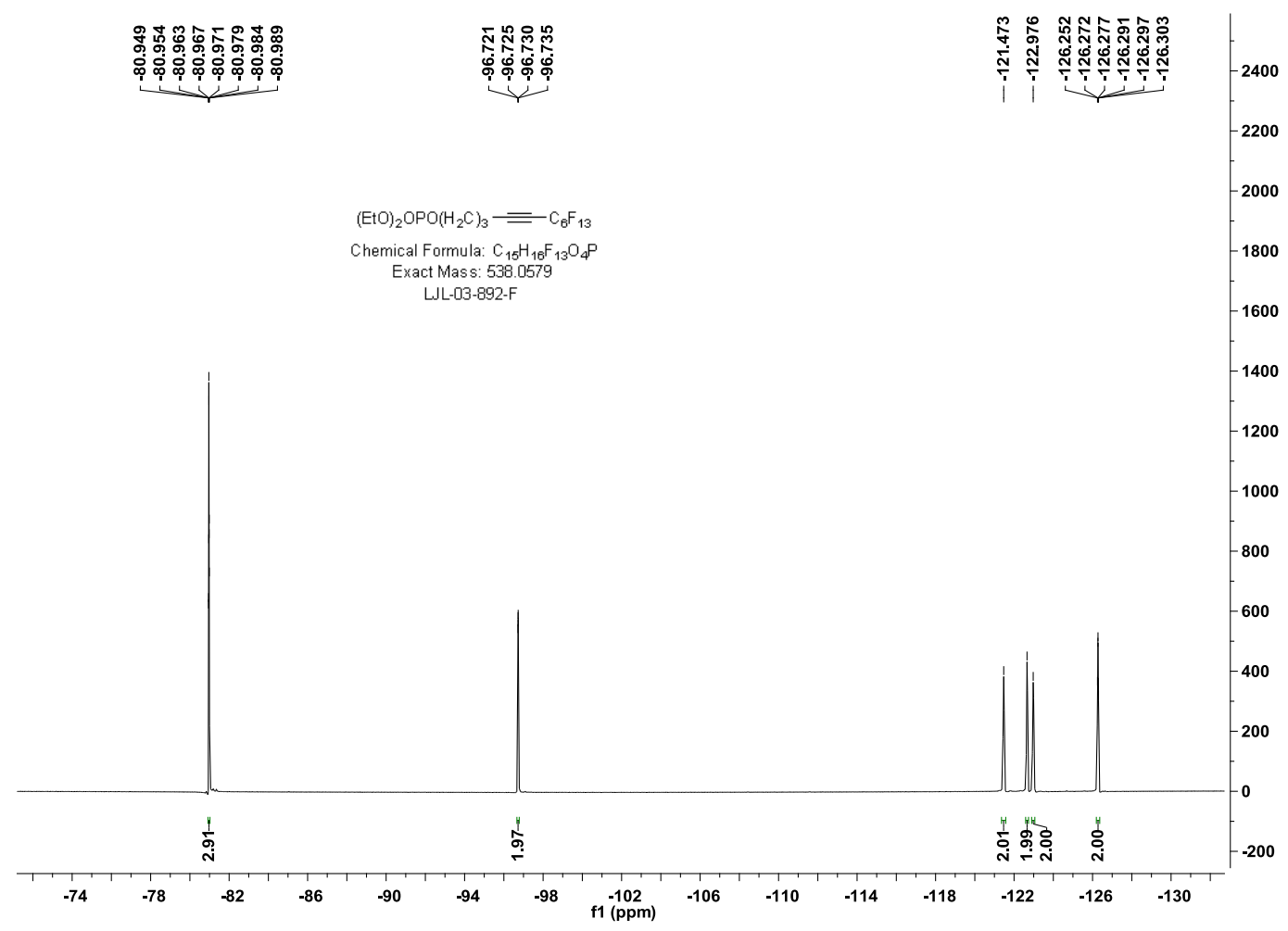

${ }^{13}$ C NMR (150.8 MHz, $\left.\mathrm{CDCl}_{3}\right)$

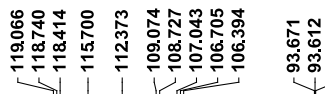

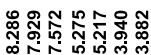

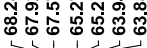

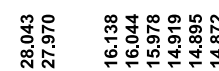

พั่

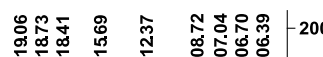

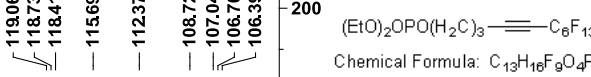
100 Exact Mass: 438.0642 $1.100 \quad$ LJL-05-892-C

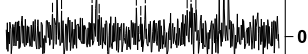

$120 \quad \begin{array}{lll}115 & 110 & 105\end{array}$

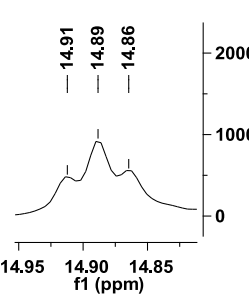

2000

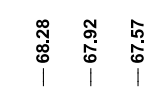

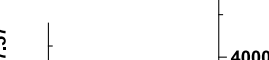

200

000

800

600

400

200

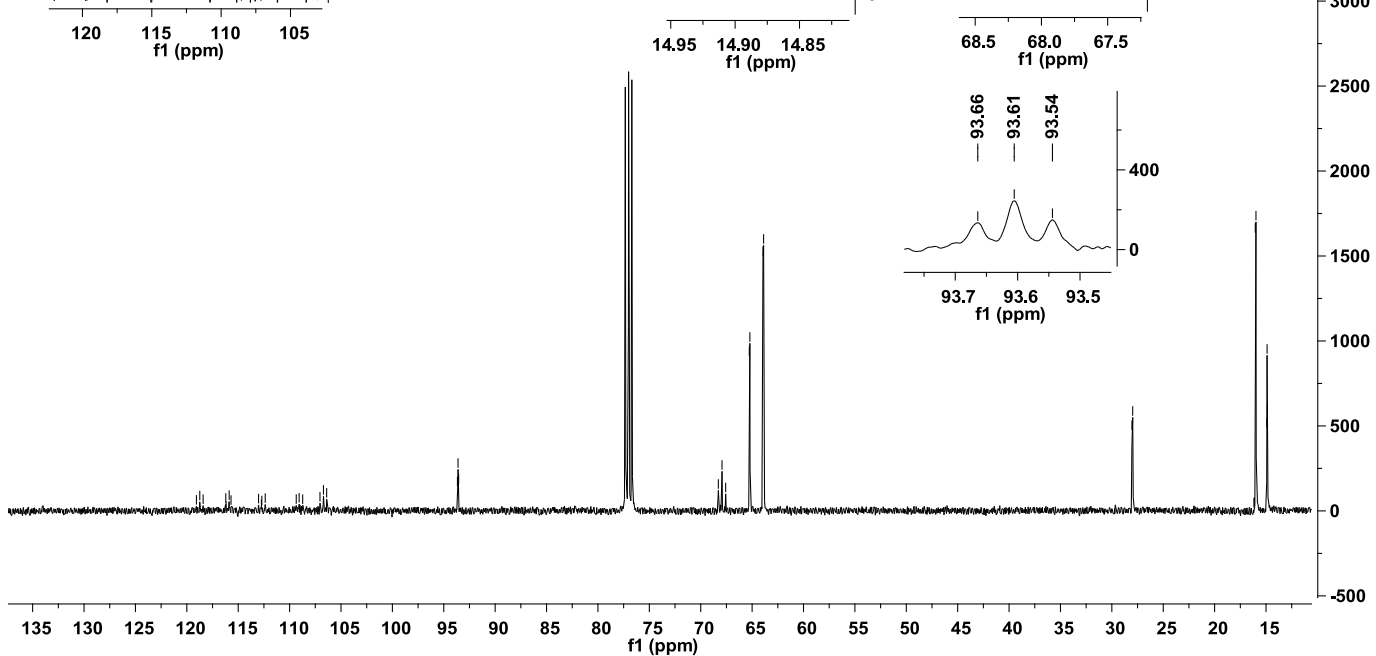


6,6,7,7,8,8,9,9,10,10,11,11,11-Tridecafluoroundec-4-yn-1-yl4-

methylbenzenesulfonate (7r)

${ }^{1} \mathbf{H}$ NMR $\left(600 \mathrm{MHz}, \mathrm{CDCl}_{3}\right)$

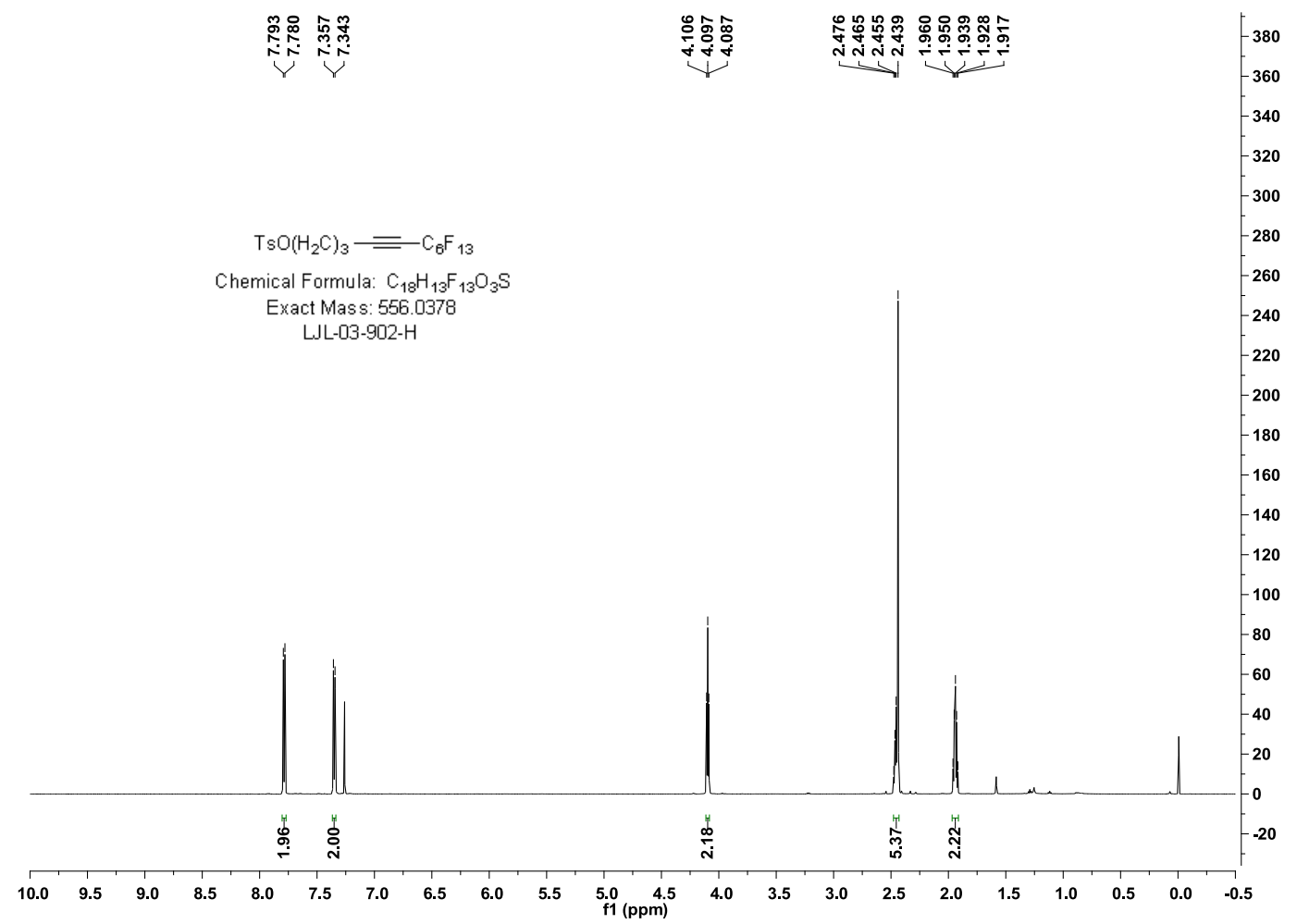

${ }^{19}$ F NMR $\left(564 \mathrm{MHz}, \mathrm{CDCl}_{3}\right)$

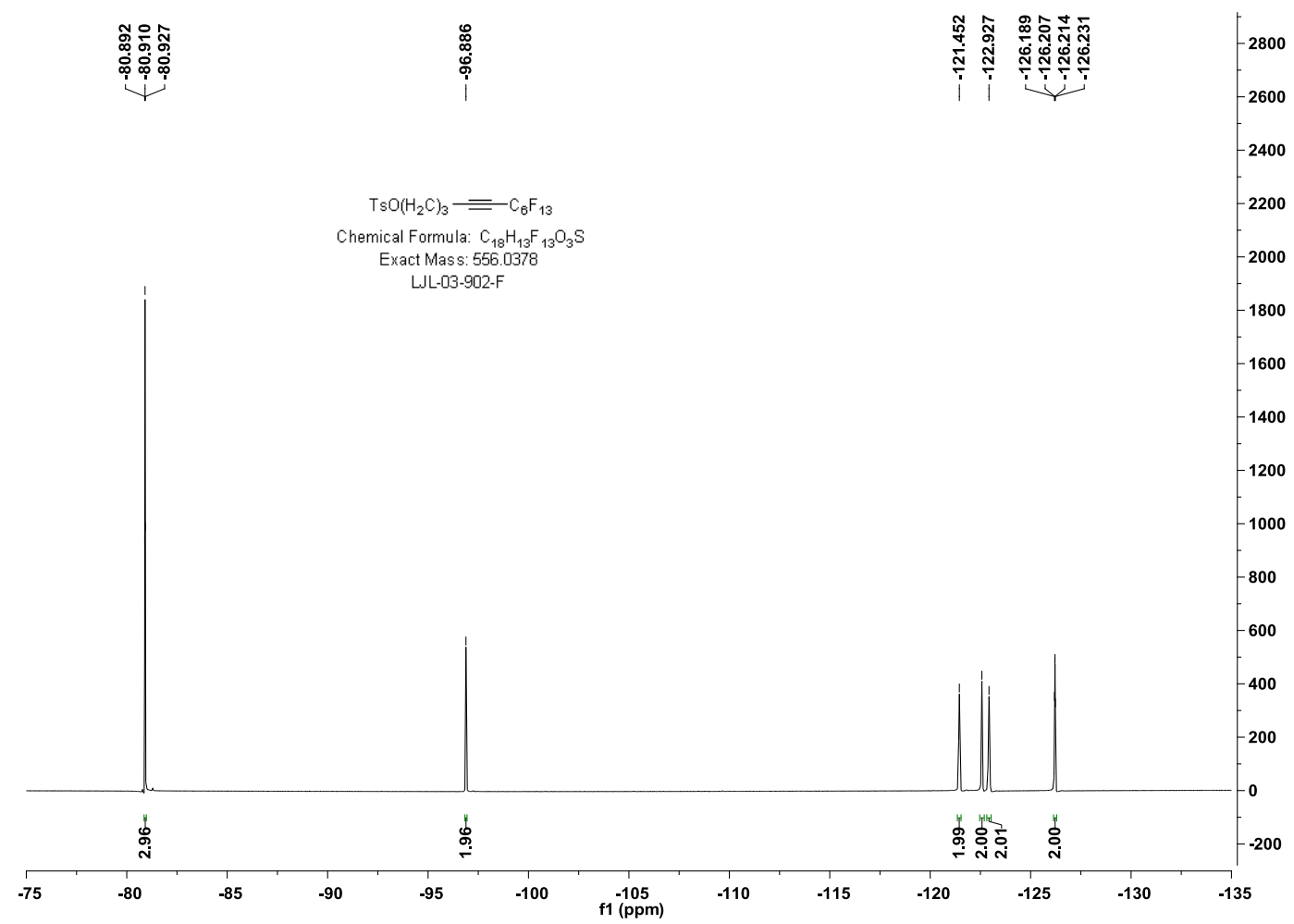


${ }^{13} \mathbf{C}$ NMR (150.8 MHz, $\left.\mathrm{CDCl}_{3}\right)$

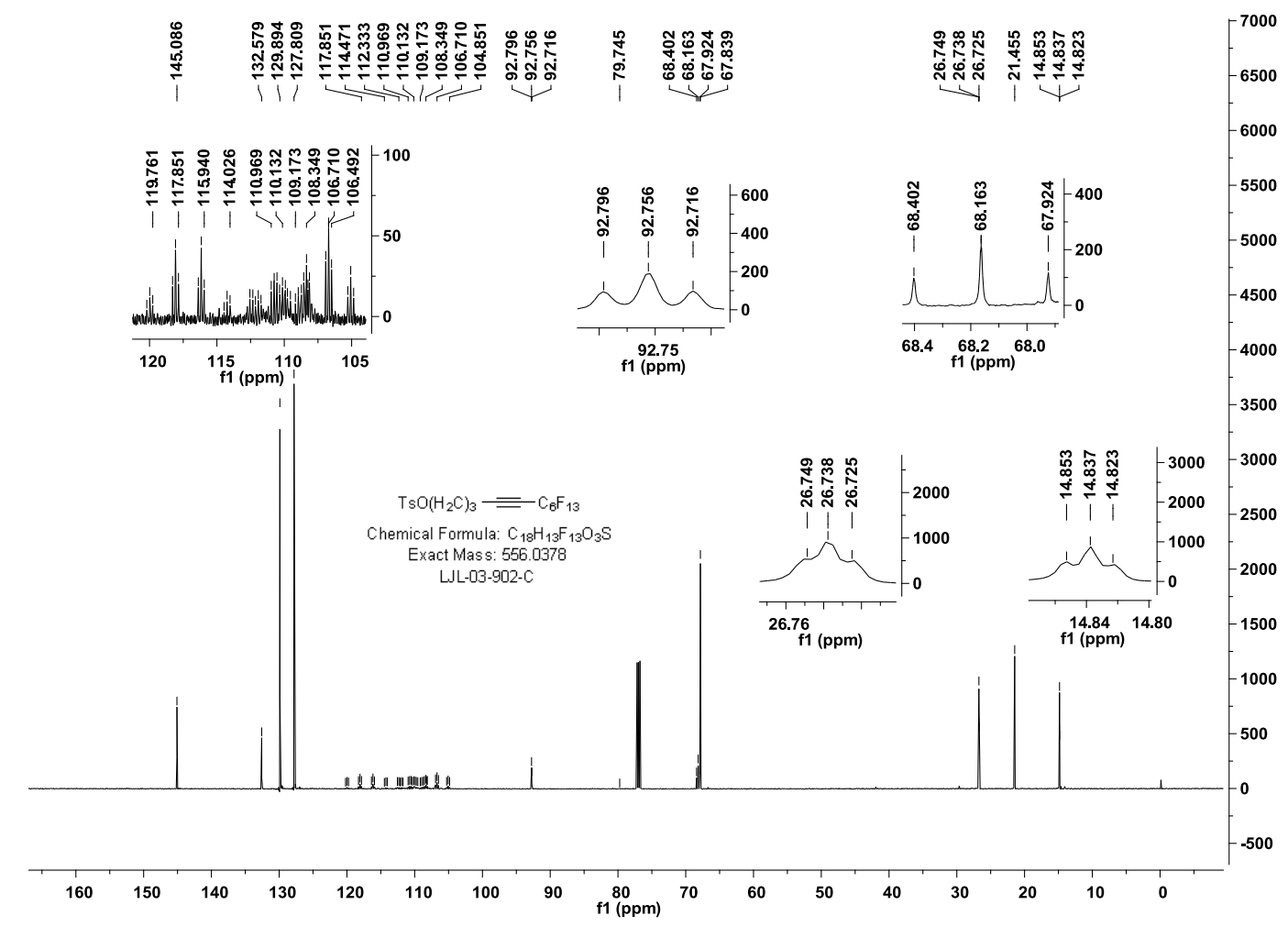

